\title{
Probability Based Load Criteria for the Design of Nuclear Structures: A Critical Review of the State-of-the-Art
}

\section{ICROI GOVER}

Prepared by M. Shinozuka, B. R. Ellingwood, P. C. Wang, C. Meyer, Y. K. Wen, S. Kao, M. L. Shooman, A. J. Philippacopoulos

Brookhaven National Laboratory

Prepared for

U.S. Nuclear Regulatory

Commission

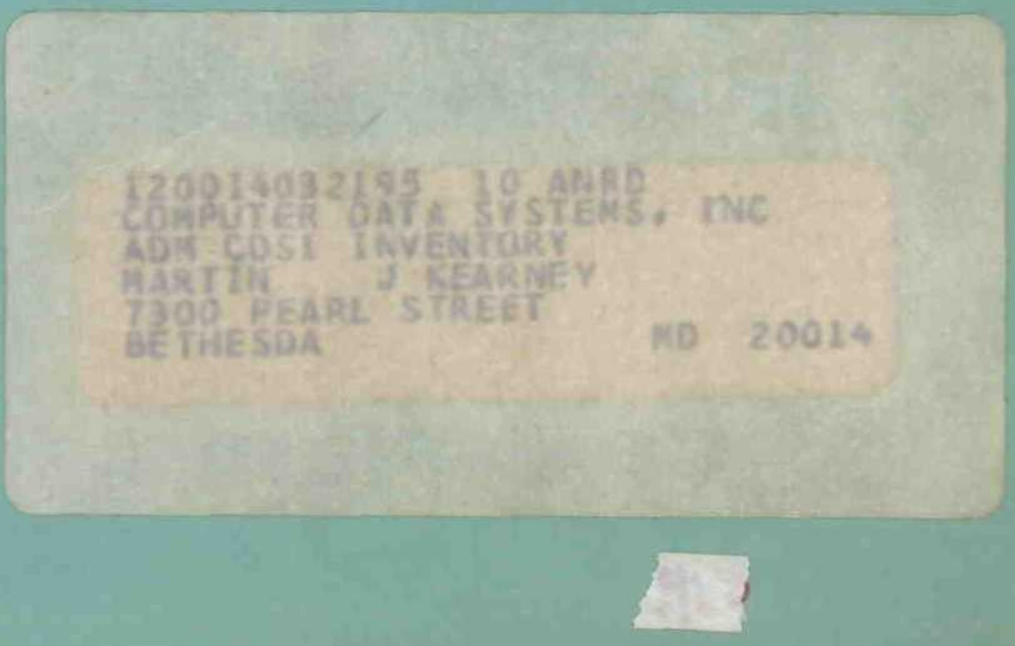




\section{NOTICE}

This report was prepared as an account of work sponsored by an agency of the United States Government. Neither the United States Government nor any agency thereof, or any of their employees, makes any warranty, expressed or implied, or assumes any legal liability or responsibility for any third party's use, or the results of such use, of any information, apparatus product or process disclosed in this report, or represents that its use by such third party would not infringe privately owned rights.

Division of Technical Information and Document Control

U. S. Nuclear Regulatory Commission Washington, D. C. 20555

Printed copy price: $\$ 7.00$

and

Nàtional Technical Information Service

Springfield, Virginia 22161 


\section{DISCLAIMER}

This report was prepared as an account of work sponsored by an agency of the United States Government. Neither the United States Government nor any agency Thereof, nor any of their employees, makes any warranty, express or implied, or assumes any legal liability or responsibility for the accuracy, completeness, or usefulness of any information, apparatus, product, or process disclosed, or represents that its use would not infringe privately owned rights. Reference herein to any specific commercial product, process, or service by trade name, trademark, manufacturer, or otherwise does not necessarily constitute or imply its endorsement, recommendation, or favoring by the United States Government or any agency thereof. The views and opinions of authors expressed herein do not necessarily state or reflect those of the United States Government or any agency thereof. 


\section{DISCLAIMER}

Portions of this document may be illegible in electronic image products. Images are produced from the best available original document. 


\section{Probability Based Load Criteria for the Design of Nuclear Structures: A Critical Review of the State-of-the-Art}

NUREG/CR--1979

Manuscript Completed: February 1981

Date Published: April 1981

\section{Prepared by}

M. Shinozuka, B. R. Ellingwood, P. C. Wang, C. Meyer, Y. K. Wen,

S. Kao, M. L. Shooman, A. J. Philippacopoulos

Brookhaven National Laboratory

Upton, NY 11973

\section{Prepared for}

Division of Reactor Safety Research

Office of Nuclear Regulatory Research

U.S. Nuclear Regulatory Commission

Washington, D.C. 20555

NRC FIN A3226 

Structures for nuclear power plant facilities must be designed to withstand safely and effectively all kinds of loads and load combinations that may be expected to occur during their lifetime. The traditional methods of structural design attempt to account for the inevitable variability in the loads, material strengths, in-service environments, and fabrication process, etc., through the use of safety factor, allowable stresses or load and resistance factors. These approaches may result in an unknown and non-uniform reliability because of the subjective manner in which the safety factors have been determined. The stochastic nature loads and the uncertainties in material properties dictate a probabilistic approach for a rational assessment of structural safety and performance.

This report presents:

(1) An in-depth review of the state-of-the-art pertaining to probabilitybased analysis and design of civil engineering structures,

(2) A basis for extending existing probability-based methods to seismic category I nuclear structures, and

(3) The availability of the pertinent data required to perform probabilistic analysis for seismic category I nuclear structures.

The report is divided into four chapters. Chapter 1 provides an introduction to the problem and discusses the objectives and scope of the investigations carried out. A summary of the findings from all chapters is also given at the end of Chapter 1 . Chapter 2 reviews current design methods for conventional as well as for nuclear structures. Unique considerations pertaining to seismic category I nuclear structures are discussed in detail. 
Chapter 3 concentrates on the probability-based design for nuclear structures. Availability of load and resistances data for probabilistic analysis is investigated. Chapter 4 details recent developments in structural reliability analysis and gives new interpretations for these theoretical developments. Alternate methods are proposed to evaluate the quantities considered to be crucial for structural reliability estimation of seismic category I nuclear structures. Some material described in this chapter goes beyond the present state of the art. Several topics supplementing various concepts mentioned in the text are also attached as appendices to the report. 


\section{ACKNOWLEDGEMENTS}

During the course of this investigation various people have contributed to the clarification of the basic issues discussed in this publication. In particular, the authors would like to acknowledge the guidance given by Dr. James Coste110 and Dr. Goutam Bagchi of RSR/NRC, during all phases of this work.

Thanks are also due to Ms. J. Murray, Mrs. E. Gilbert and Ms. I. C. Frank for preparing the various versions of this report. 
$$
\text { . }
$$ 
ABSTRACT i i i

ACKNOWLEDGEMENTS

LIST OF TABLES $x$

LIST OF FIGURES $x i$

1. EXECUTIVE SUMMARY

1.1 Introduction

1.2 Objectives

1.3 Scope

1.4 Summary and Conclusions of Chapter $2 \ldots \ldots \ldots \ldots \ldots . . .3$

1.5 Summary and Conclusions of Chapter $3 \ldots \ldots \ldots \ldots \ldots .1-5$

1.6 Summary and Conclusions of Chapter $4 \ldots \ldots \ldots \ldots \ldots .10$

2. GENERAL COMMENTS REGARDING CURRENT STRUCTURAL DESIGN PROCEDURES

2.1 Design of Conventional Structures $\ldots \ldots \ldots \ldots \ldots \ldots \ldots .2-1$

2.2 Design of Nuclear Structures $\ldots \ldots \ldots \ldots \ldots \ldots \ldots \ldots .2-4$

2.3 Special Considerations for Nuclear Structures $\ldots \ldots \ldots .2-12$

2.3.1 Consequence of Faiture $\ldots \ldots \ldots \ldots \ldots \ldots \ldots \ldots \ldots \ldots, 2-12$

2,32 Loading Conditions Unique to Nuclear Structures $\ldots \ldots \ldots$ 2-13

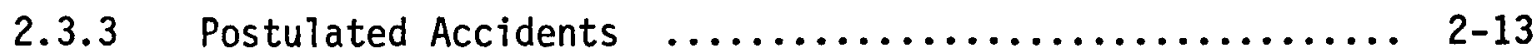

2.3.4 Complexity and Difficulty in Decomposing the Structural

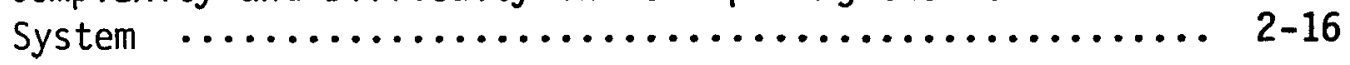

2.3.5 The Design Process and Apportionment of Subsystem

Reliabilities ............................... 2-16

3. PROBABILITY-BASED DESIGN FOR NUCLEAR STRUCTURES

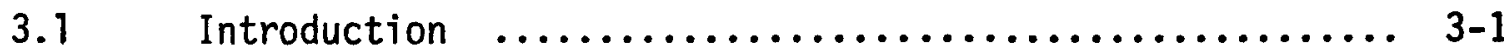

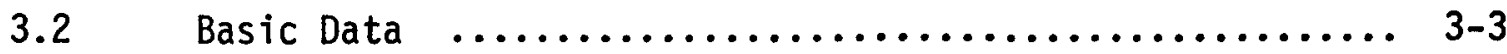

3.2.1 Structural Loads $\ldots \ldots \ldots \ldots \ldots \ldots \ldots \ldots \ldots \ldots \ldots \ldots . . \ldots \ldots$

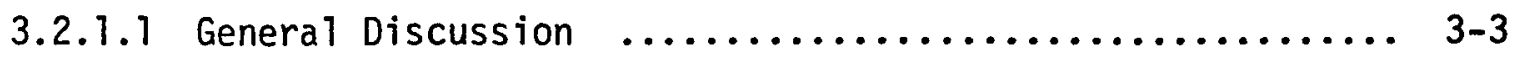


3.2.1.2 Dead Load 3-4

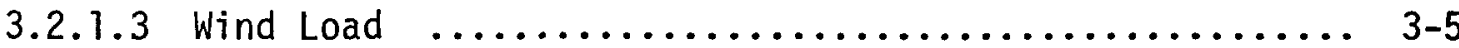

3.2.1.4 Tornado Load $\ldots \ldots \ldots \ldots \ldots \ldots \ldots \ldots \ldots \ldots \ldots \ldots . . \ldots \ldots$

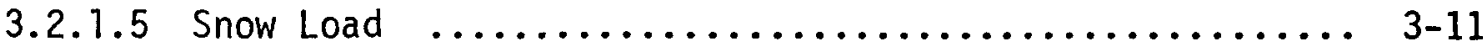

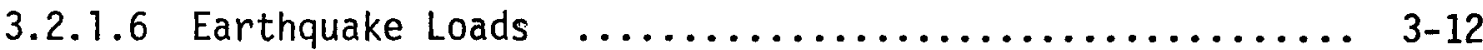

3.2.1.7 Limitations of Environmental Load Data ............ 3-15

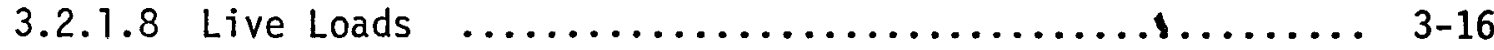

3.2.1.9 SRV Discharge Loads $\ldots \ldots \ldots \ldots \ldots \ldots \ldots \ldots \ldots \ldots \ldots \ldots \ldots \ldots \ldots, 17$

3.2.1.10 Abnormat Loads ............................ 3-18

3.2.2 Structural Resistance $\ldots \ldots \ldots \ldots \ldots \ldots \ldots \ldots \ldots \ldots .3,20$

3.2.2.1 General Discussion $\ldots . \ldots \ldots \ldots \ldots \ldots \ldots \ldots \ldots \ldots . . . \ldots \ldots$

3.2.2.2 Hot-Rolled Structural Steel $\ldots \ldots \ldots \ldots \ldots \ldots \ldots \ldots . . .23$

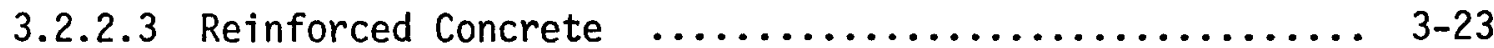

3.2.2.4 Limitations of Existing Resistance Data $\ldots \ldots \ldots \ldots . . .3-24$

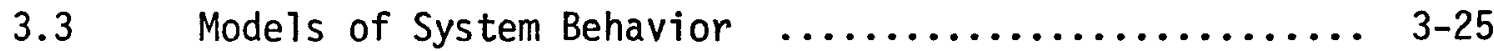

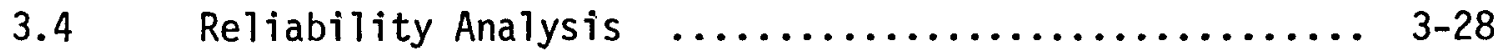

3.4.1 System and Component Reliability $. . . \ldots \ldots \ldots \ldots . . .63-28$

3.4.2 Interpretation of Small Probabilities $\ldots \ldots \ldots \ldots . . .3-30$

3.4.3 Measures of Reliability ..................... 3-30

3.4.4 Indentification of Failure Events $\ldots \ldots \ldots \ldots \ldots \ldots .3-31$

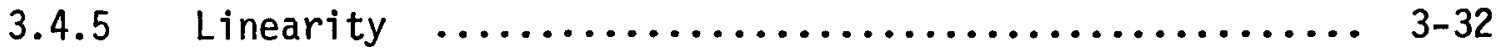

3.5 Specification of Safety Criteria $\ldots . \ldots \ldots \ldots \ldots \ldots . . . . .33$

3.5.1 Target Reliabilities $\quad . . \ldots \ldots \ldots \ldots \ldots \ldots \ldots \ldots \ldots . . \ldots \ldots$

3.5.2 Code Formats $\ldots \ldots \ldots \ldots \ldots \ldots \ldots \ldots \ldots \ldots \ldots \ldots \ldots, 3-35$

3.5.3 Design Loads $\ldots . \ldots \ldots \ldots \ldots \ldots \ldots \ldots \ldots \ldots \ldots \ldots . . \ldots \ldots$

3.5.4 Design Philosophy $\ldots \ldots \ldots \ldots \ldots \ldots \ldots \ldots \ldots \ldots \ldots . .6 \ldots$ 
4. ANALYSIS OF RELIABILITY OF STRUCTURES

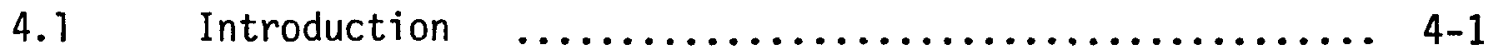

4.2 First-Order, Second-Moment Methods $\ldots \ldots \ldots \ldots \ldots . . . .4$ 4-3

4.3 Method of Lagrange Multipliers .................. 4-10

4.4 Dependence of $\beta$ on Distribution Functions $\ldots \ldots \ldots \ldots$ 4-17

4.5 Design Point as the Point of Maximum Likelihood ...... 4-20

4.6 Estimation of Limit State Probabilities ........... 4-25

4.7 Second Order Higher Moment Methods and General Forms of Probability Distribution and Density Functions ..... 4-32

4.7.1 Second Order Higher Moment Methods $\ldots \ldots \ldots \ldots \ldots \ldots .4$ 4-32

4.7.2 General Forms of Probability Distribution and Density

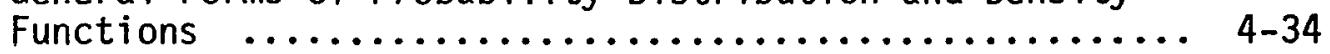

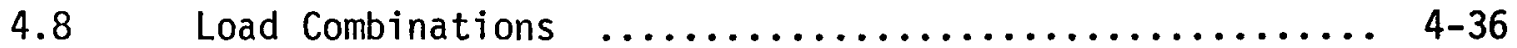

4.8.1 Introduction $\ldots \ldots \ldots \ldots \ldots \ldots \ldots \ldots \ldots \ldots \ldots \ldots, 4,36$

4.8.2 Combinations of Random Load Effects .............. 4-38

4.8.3 Bounding Techniques and Other Approximations ........ 4-41

4.8.4 Load-Coincidence Method ...................... 4-51

4.8.5 Method of Laplace Transform and Renewal Equation ..... 4-54

4.8.6 Errors of Some Load Combination Rules ............. 4-56

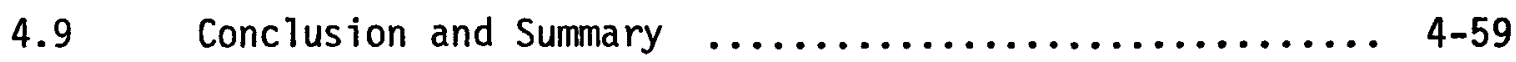

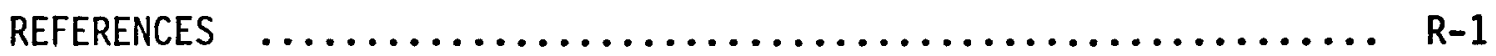

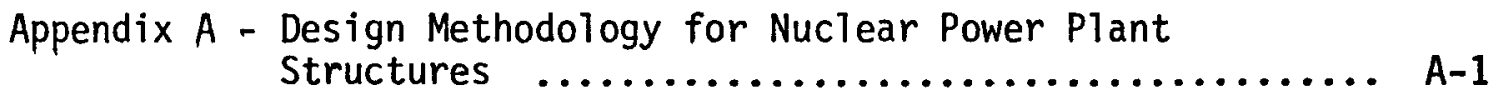

Appendix B - Consensus Estimation Procedures ............... B-1

Appendix C - System Reliability Modeling Methods ............ C-1

Appendix D - Second Order Higher Moment Approximation ........ D-1

Appendix E - General Forms of Probaiblity Density Functions .... E-1 
Appendix F - Bounded Tail Probabilities Using Inequalities of the Chebycheff Type $\ldots \ldots \ldots \ldots \ldots \ldots \ldots \ldots \ldots \ldots \ldots$. F-1

Appendix G - Comments on the Application of Upcrossing Methods to Load Combinations .......................... G-1

Appendix $\mathrm{H}$ - Notes on the Combinations of Random Loads I $\ldots . \ldots \ldots$ H-1

Appendix I - Notes on the Combinations of Random Loads II ........ I-1 


\section{LIST OF TABLES}

Table

Title

Page

2.1

Current Design Practice for Nuclear Structures

3.1 Availability of Structural Load Data for

Probability Analysis

Typical Basic Statistics for Steel Properties

3.3

Typical Resistance Statistics for Steel Structural

Members

Typical Basic Statistics for Reinforced Concrete Elements

Typical Resistance Statistics for Reinforced

Concrete Structural Members 


\section{LIST OF FIGURES}

Figure

Title

Page

3.1

Mark III Containment

4.1 Illustration of the Reliability Index Concept

Formulation of Safety Analysis in Original and Standardized Variable

4.3 Determination of Design Point When Components

$$
\text { of } \underline{x} \text { are Uncorrelated }
$$

4.4 Determination of Design Point When Components

$$
\text { of } \underline{x} \text { are Correlated } \quad 4-70
$$

4.5 Limit State Surface in the $x-z$ Space 4-71

4.6 Use of Contour Integral for the Estimation of the Limit State Probabilities 4-72

4.7 Limit State Under Multiple Limit Conditions 4-73

4.8 Schematic Representation of Various Loads and Load Effects 


\section{Executive Summary}

\subsection{Introduction}

Structures for nuclear power plant facilities must be designed to withstand safely and effectively all kinds of loads and load combinations that may be expected to occur during their lifetime. These loads include various static and dynamic loads which are caused by operational, environmental and accidental conditions. The traditional methods of structural design attempt to account for the inevitable variability in the loads, material strengths, in-service environments, and fabrication process, etc., througn the use of safety factor, allowable stresses or load and resistance factors. These are prescribed in the various standards and specifications developed by such groups as ASME, ACI, AISC, etc. and the NRC Standard Review Plan (SRP). However, the subjective manner by which these safety factors have been determined result in an unknown and nonuniform reliability (where reliability is defined as the probability that the structure will not fail during the projected lifetime).

The stochastic nature of loads and the uncertainties in material properties dictate a probabilistic approach for a rational assessment of structural safety and performance. This means that the structures should be designed according to a required level of reliability. In recent years, an increasing effort has been directed toward the application of reliability analysis in conventional structures. In the nuclear industry also, there has been an increasing trend toward the utilization of the probabilistic-based design and reliability analysis for safety evaluations. 
A program entitled "Load Combinations for Design of Category I Structures" was initiated in mid-year 1980. The overall aim of this program is to develop a rational approach based on the probabilistic design methodology for safety evaluation of reactor containments and other seismic category I structures subjected to multiple loadings. This report summarizes the work carried out since the initiation of the program, i.e., from April 1980 to the end of December 1980.

\subsection{Objectives}

The objectives of this report are to:

(1) Present an in-depth review of the state-of-the-art pertaining to probability-based analysis and design of civil engineering structures,

(2) Present a basis for extending existing probability-based methods to seismic category I nuclear structures, and

(3) Determine the availability of the pertinent data required to perform probabilistic analysis for the seismic category I nuclear structure. The latter two objectives are added for the sake of completeness, although they go somewhat beyond original contractual commitments.

\subsection{Scope}

This report is divided into four chapters. The present chapter, Chapter 1 , is intended to provide an introduction to the problem and to touch upon the objectives and scope of the investigations carried out for this report. A summary of the findings from all chapters is also given at the end of Chapter 1 . Chapter 2 reviews current design methods for conventional as well as for nuclear structures. Unique considerations pertaining to seismic category I nuclear structures are discussed in detail. Chapter 3 concentrates on the probability-based design for nuclear structures. Availability of load and resistance data for probabilistic analysis is investigated. Chapter 4 details recent 
developments in structural reliability analysis and gives new interpretations for these theoretical developments. Alternate methods are proposed to evaluate the quantities considered to be crucial for structural reliability estimation of seismic category I nuclear structures. Some material described in this chapter goes beyond the present state of the art. Several topics supplementing various concepts mentioned in the text are also attached as appendices to the report.

\subsection{Summary and Conclusions of Chapter 2}

Chapter 2 is intended to summarize the current design and safety evaluation procedures used for conventional as well as nuclear structures. Special considerations pertaining to nuclear structures in terms of consequences of failure, uniquely loading conditions, and their implications for probability based design are discussed in detail. A brief summary of the chapter highlights is given below.

1. For conventional structures, either an allowable stress approach or an ultimate strength approach has been used for design and safety evaluations. To account for the uncertainties in loads and material strengths, the allowable stress approach utilizes a "safety factor" concept, while the ultimate strength approach is based on "load and resistance factor" concepts. Both approacfies result in unknown and nonuniform safety levels because of the subjective manner in which the safety factors have been determined. In recent years, probability-based design approaches have been developed with the aim of achieving consistent reliabilities for various limit states. At the present time, these methods are applicable only to individual simple components, such as beams, and columns. With few exceptions, they have not as yet been applied to the system as a whole.

2. For design and safety evaluation of nuclear structures, the concept of safety factors or load and resistance factors is also widely used. 
The design requirements for seismic category I structures, however, are much more complex than those for conventional structure. The requirements are detailed in the NRC Standard Review Plan (SRP), which is based on standards, developed by such organizations as ASME, ACI, AISC, etc. (see Table 2.1).

It is to be noted however, that the determination of the various factors used for the nuclear structures relies heavily on experience in the design and construction of ordinary structures. The extension of the factors to nuclear structures does require further verification. For example, a load factor of 1.7 is applied to equipment live loads, normal thermal loads, and pipe reaction loads, despite the fact that (a) the character of live loads in nuclear structures may be quite different than those acting on ordinary structures, and (b) thermal effects and pipe reactions bear very little similarity to live loads. Other examples of inconsistencies with regard to load and resistance factors are also discussed in the text. In view of this, it seems that it is difficult to achieve consistent levels of reliability using the currently prescribed methods. Such a consistency however, is imperative for nuclear structures. The probability-based design can achieve such a goal, and thus it should be pursued.

3. The design of seismic category I nuclear structures requires many unique considerations. Some of the most important items are: (1) the severe consequence of failure, (2) safety-related functional limit states, such as the unexpected release of radiation, (3) the unique postulated loadings, e.g., LOCA, SRV, etc. specific to nuclear structures, (4) lack of sufficient data and experience for some loading conditions, e.g., operating live load and abnormal loads, (5) required high levels of reliability, (6) and the complexity in decomposing the structural system into subsystems and the apportionment of reliabilities thereof. 


\subsection{Summary and Conclusions of Chapter 3}

Chapter 3 presents an outline of the necessary items for the development of reliability-based design criteria. Specifically, the following task outline is suggested:

a. Develop necessary data base to determine occurrence frequencies and intensities of various environmental and postulated loads and resulting structural responses.

b. Establish models to describe the behavior of the structural system under prescribed loading conditions.

c. Establish procedures for computing reliability of different elements, components and of the structural system.

d. Specify safety checking procedures to be used by the structural designer.

While each of the above tasks has reliability features common to ordinary structures, there are nevertheless many aspects that are unique to nuclear structures. The contents of this chapter is written with a twofold purpose, namely; (1) to highlight existing research upon which standards for the design of ordinary structures are based and which appear to be immediately applicable for nuclear structures, and (2) to point out problem areas where additional efforts appear necessary. Items 1 to 6 below summarize some of the chapter highlights. 
1. The various static and dynamic loads on the nuclear structures may be caused by operational, environmental, and abnormal conditions. They are discussed in detail in the text. The availability of the pertinent load data is shown below.

Load Type

Dead

Operating live

Snow

Ice

Rain

Wind

Extratropical

Hurricane

Tornado

Pressures

Pressure drop

Missiles

Earthquake

SRV

Abnormal

\section{Status Code}

1

3

2

2

2

1

2

2

3

3

2

2

3

\section{Status code}

1. Ready to use in probability based design.

2. Raw data available, some further analysis required.

3. Raw data unavailable or of questionable quality. 
2. The relationship between load and load effect can be represented by the equation $Q_{j}=C_{j} B_{j} A_{i}$. Where $Q_{j}$ is the load effect. All three terms $A_{i}, B_{i}$, and $C_{i}$ are random variables. $A_{i}$ is the basic load. The type of loads referred to by the term $A_{i}$ and their availability was summarized under Item 1 above. $B_{i}$ is essentially a modeling parameter that is used to transform actual varying loads into idealized loads for structural analysis. An example of $B_{i}$ would result from the approximation of a live load into an equivalent static loads. Finally, $c_{j}$ is a structural analysis factor arising from the structural modeling process. For example, the modeling of an actual three-dimensional structure by an idealized axisymmetric finite-element model. Since all of the above terms affect the probabilistic results, their variation are under investigation. In this chapter some results pertaining to $A_{j}$ and $B_{i}$ are presented.

3. Over the past decade a considerable amount of resistance data for structural members has been gathered. Relevant data for reinforced concrete, structural steel and beams and columns made from reinforced concrete and steel are given in Tables $3.2-3.5$ of the text. Although this data base pertains to grades of concrete and structural steels common to ordinary structures, their statistics should nevertheless be valid (in most cases) for reliability analysis of nuclear structures. Further investigation of resistance data for rapidly applied loads should be undertaken. The extension of the data base to include other structural members, such as plate and shell will also be necessary. 
4. Methods for structural system modeling have undergone substantial development during the last decade. As mentioned the parameter $c_{\mathbf{j}}$ depends on the accuracy of the system modeling. For seismic category I nuclear structures, the structure is generally composed of a very elaborate geometry configuration which is idealized by finite-elements. Various proprietary as well as publicly available computer codes are available and have been used extensively for both static or dynamic evaluation of structures undergoing elastic as well as inelastic response. The accuracy of these models depends on (1) the finite element grid size, (2) the idealization of the structural and boundary conditions, (3) assumptions made in linearization of nonlinear equations, (4) assumptions of damping and number of modes used for dynamic analysis, and (5) the time-steps and dynamic reduction schemes chosen for time dependent problems. While the development of finiteelement methods has progressed to the point that most of the codes available for the industry should be sufficiently accurate, there is a need for code verification by analytical and physical test benchmarks. An effort to determine variations caused by some of the above mentioned items will be made.

5. The component as well as system reliability must be considered for nuclear structures because of their complexity. The methods for system reliability are less well defined and additional efforts that relate system reliability to element reliability will be persued. 
6. The study carried out for this report reveals that practical probabilitybased design criteria can be developed. In principle, the criteria need not be more complicated to apply than existing criteria. Indeed, as discussed in the text, simplification of the existing criteria may be possible. These newly developed criteria based on the reliability analysis and design will have a well-established rationale. A number of different formats for the design equations (checking formula) are feasible. These will facilitate the integration of the probability-based criteria into the appropriate sections of the Standard Review Plan. 


\subsection{Summary and Conclusions of Chapter 4}

In Chapter 4, some of the more significant recent developments in the area of structural reliability analys is are reviewed, new interpretations for the analytical procedures involved in these developments are proposed where appropriate, and efforts are made to advance the current state of the art wherever possible within the constraint of the resources available. The major findings of this chapter are indicated below.

1. The basic premise under which the structural reliability analysis is performed can be described as follows. A mathematical model is first derived from principles of mechanics and experimental data. The model describes a relationship among the resistance and load variables $x_{1}, x_{2}, \ldots$, $x_{n}$ under operating, accidental and environmental loading conditions. This relationship can be written in the form of an inequality known as the limit state equation $g\left(x_{1}, x_{2}, \ldots, x_{n}\right) \leq 0$. If the resistance and load variables assume values which satisfy the limit state equation, the corresponding limit state (e.g., the state of excessive stress at a structural location) has been reached. The probability that the limit state will be reached during the lifetime of the structure is then called the limit state probability, while the probability that the state will not be reached is called the reliability of the structure.

2. The so-called advanced First Order Second Moment (FOSM) methods are found to be useful for providing the theoretical foundation for the reliability-based design codes and for developing the load combination formats based thereupon. The reliability index is used in the advanced FOSM methods to reflect the limit state probability. The index, however, has a much greater theoretical significance than that associated with the mean-value 
based FOSM method. Also, it reflects more accurately the limit state probability, since the advanced FOSM methods can account for the generally nonnormal nature of the resistance and load variables. In fact, the non-normal nature of these variables can be accounted for in the advanced FOSM methods only if their distribution functions are fully known. In principle, this makes it possible to pursue the full-distribution-based reliability analysis, and thus the reliability index and the limit state probability are uniquely related, at least implicitly, in the advanced FOSM methods.

3. When the resistance and load variables $x_{1}, x_{2}, \ldots, x_{n}$ can be idealized as random variables, the so-called design point $x_{1}^{*}, x_{2}^{\star}, \ldots, x_{n}^{\star}$ is determined by the advanced FOSM methods. The advanced FOSM methods are stipulated to include a more straightforward method where the design point is determined as the point of maximum likelihood at which the joint density function of $x_{1}, x_{2}, \ldots, x_{n}$ assumes its maximum value. In any case, the design point is on the limit state surface $g\left(x_{1}^{\star}, x_{2}^{*}, \ldots, x_{n}^{*}\right)=0$ and depends on the postulated limit state equations and the target value of the safety index (or the limit state probability) to be assigned to the limit state. 4. By expressing $x_{i}^{*}$ in terms of their corresponding nominal values $x_{N_{i}}$, one obtains $x_{i}^{*}=\gamma_{i} X_{N_{j}}$ where the factors $\gamma_{i}$ are defined as the load or resistance factors. This leads to the widely used linear load combination formats, if the limit state function $g\left(x_{1}, x_{2}, \ldots, x_{n}\right)$ is a linear function of $x_{1}, x_{2}, \ldots, x_{n}$. Since the design point depends on the (postulated) limit state and the safety index (or the limit state probability) while the nominal values $X_{N_{i}}$ are usually fixed, the load and resistance factors depend on the limit state to be postulated as well as on the target safety index (or target limit state probability) to be assigned. Presently, however, it is not quite clear, how one can postulate specific limit states 
and at the same time assign target limit state probabilities. This is an important subject for future study.

5. For a structure subjected to multiple time-varying loads, it is recommended that (a) load effects $Y_{i}(t)$, rather than loads $X_{j}(t)$, be used to construct the limit state equation, (b) load effects $Y_{j}(t)$ be assumed to be linearly related to loads $X_{j}(t),(c)$ total load effect $Y(t)$ be as sumed to be the sum of the component load effects $Y_{i}(t) ; Y(t)=\sum_{i=1}^{N} Y_{i}(t)$, (d) the limit state equation be of linear form, $R-Q \leqq 0$, with $R$ representing the structural resisting capacity while $Q=Q_{T}$, the maximum of $Y(t)$ during the lifetime $T$ of the structure.

6. The total load effect $Y(t)$ under consideration may consist of component effects which are time-invariant except for one, say, $Y_{N}(t)$. Then, $Y_{i}(t)$ may be considered as the random variables $Y_{i}(i=1,2, \ldots, N-1)$. Generally, the distribution function of $Q_{N T}$ representing the maximum value of $Y_{N}(t)$ during the lifetime $T$ of the structure can be estimated empirically or analytically at least in approximation. Then, the lifetime maximum $Q=$ $Q_{T}$ of $Y(t)$ can be written as $Q=Q_{N T}+\sum_{i=1}^{N-1} Y_{i}$, and the limit state equation becomes $R-Q_{N T}-\sum_{i=1} Y_{i} \leqq 0$. Since this limit state equation involves random variables only, the safety index $\beta$ can be computed with the aid of the FOSM methods, the limit state probability obtained from full-distribution procedures and the design point determined. The load and resistance factors can then be determined in the manner described above.

7. The determination of load and resistance factors on the basis of either the FOSM methods or full-distribution procedures requires that the design point associated with the limit state equation be identified. Present techniques do not permit this if two or more load effects must be idealized as random processes rather than as random variables. In this case, one 


\section{GENERAL CO:MMENTS REGARDING CURRENT STRUCTURAL DESIGN PROCEDURES}

\subsection{Design of Conventional Structures}

The design process for conventional structures involves checking a safety criterion in the form.

Design Resistance $\geq$ Effect of Design Loads

to insure satisfactory structural performance. A7though a number of "deemed to satisfy" clauses (e.g., minimum width-thickness or maximum span-depth ratios) must also be satisfied, the safety criterion of the form of Eq. 2.1 above is the main concern. The resistance corresponds to a particular limit state such as yielding of a tensile member, buckling of the compression flange of a beam, or the lateral instability of a column. The loads arise from a variety of sources, such as the environment, operations and accidents.

Specifications governing design for different construction materials usually involve different philosophies and thus Eq. 2.1 would be handled differently. For instance, when specifications are based on the allowable stress design philosophy, the design resistance is computed using a stress which is a preset fraction of the yield stress, modulus of rupture, buckling stress or some other appropriate failure stress. The loads are considered to be close to the maximum probable loads that could occur at some interval of the useable life-time of the structure and hence elastically computed stresses arising from these loads should not exceed the allowable stress. For a simple tension member subjected to dead and live load with the resulting effects, $D_{\text {eff }}$ and $L_{\text {eff }}$, Eq. 2.1 becomes,

$$
A F_{a}=A F_{y} / F S>D_{e f f}+L_{e f f}
$$

in which $F_{a}, F_{y}=$ allowable tensile stress and yield stress, respectively, 
and $A=$ member area. The ratio of the failure stress to allowable stress is the overall factor of safety, FS. An example of such provisions are found in Part I of the American Institute of Steel Construction (AISC) Specifications [1978].

In strength design, the loads are multiplied by load factors, and the calculated strengths (e.g., moment capacity) are reduced by resistance factors which are less than or equal to unity. For the simple tension member used in the example above, Eq. 2.1 would be,

$$
\phi F_{y} A>\gamma_{D} D_{\text {eff }}+\gamma_{L} L_{\text {eff }}
$$

in which $\phi=$ capacity or resistance factor and $\gamma_{D}, \gamma_{L}=$ load factors. Such provisions are found in American Concrete Institute Standards 318 [1977] and $349[1976]$.

The provision of allowable stresses, or of load and resistance factors is an implicit recognition by specification writers of the nondeterministic nature of the structural resistances and loads, and the need to account for uncertainties inherent in the analysis and design process. These generally conservative load and resistance factors or allowable stresses reflect the profession's concern regarding possible unfavorable deviations in the load and resistance variables from the values specified for design. In the case of reinforced concrete, the assignment of a larger load factor to live load than to dead load is due to the recognition that there is a larger uncertainty in the magnitude of time-varying loads than the uncertainty inherent in the permanent loads. This represents an additional step beyond the lumping of all uncertainties in one overall safety factor or in the fraction used to 
compute the allor:able stress. However, in all cases these factors have been set empirically through subjective engineering judgment by specification committees. Adjustments of these factors in subsequent revisions in response to new desiqn environments can be based on experience with structures conforming to existing and past specification. Generally, such adjustments tend to be minor

This approach of setting safety criteria has, for the most part, served the engineering profession well in the past. Conservative results have usually been achieved for traditional construction materials with which there is considerable experience. There are some problems with using such subjectively determined criteria, however. It is difficult to assure that the design criteria are consistent with the performance objectives of the specification group developing them. Constant levels of reliability are difficult to achieve for all structures covered by the specification if the safety factors are set subjectively. Moreover, when heavy reliance is placed on experience in selecting the safety factors, there is no assurance that these factors will be appropriate for designs calling for different construction materials than those explicitly covered by the subjective as well as the objective data base. These problems become even more relevant in the design of nuclear structures and will be discussed in the subsequent sections. 


\subsection{Design of liuclear Structures}

The current design specifications deal with the various loads and load categories for nuclear structures in rather differing ways.

For metal containments, the pertinent ASME Code Section III, Division 1 does not define explicitly loads and load categories, but only certain levels of service limits which indirectly correspond to classes of loads or load combinations of increasing severity.

Level A Service Limits - Service loadings to which the containment is subjected, including the plant or system design basis accident condition (DBA) for which the containment function is required;

Level B Service Limits - Loads subject to Level A Service Limits plus the additional loads resulting from natural phenomena during which the plant must remain operational;

Level C Service Limits - Loads subject to Level A Service Limits plus the additional loads resulting from natural phenomena for which safe shutdown of the plant is required;

Level D Service Limits - Other applicable service limits and loadings of a local dynamic nature for which the containment function is required.

The code makes reference also to testing conditions in conjunction with hydrostatic or pneumatic lests including leak tests, which normally simulate operating or design basis accident conditions. 
For concrete containuents, the ASME Code Sution III, Division 2 defines explicit and precise load categories. These are characterized by their sources, increasing magnitude or severity, and decreasing probability of occurrence, or increasing recurrence period. This should be measured in terms of the structure design life, which is normally assumed to be thirty to forty years. The load categories are defined as follows.

\section{Construction Category}

Loads in this category include cranes and other equipment loads, scaffolding, wet concrete, prestressing, creep and shrinkage, and wind and are essentially comparable to construction loads in conventional construction.

2. Test Category

Newly completed containment buildings and reactor pressure vessels have to undergo acceptance testing to verify structural integrity and leak tightness. The loads to be considered are an initial proof test pressure, $P_{t}$, and the test temperature, $T_{t} \cdot$ The significance of these loads is that their magnitudes are wellcontrolled while intended to simulate normal operating and design basis accident conditions.

3. Normal Load Category

This category includes all loads due to plant start-up, normal operation and scheduled shutdown for refueling and maintenance service. These are: 
D - dond loads, including hydrostatic alld permanent cquipment róad

L - live loads, including any movecuble cipuipment loads and other loads which vary with intensity and occurrence (e.g., soil pressure)

$F$ - loads resulting from the application of prestress

$T_{0}$ - thermal effects and loads during normal operation or shutdown condition, based on most critical transient or steadystate condition

$R_{0}$ - pipe reactions during normal operation or shut-down condition, based on most critical transient or steady-state condition

$P_{v}$ - external pressure loads resulting from pressure variation either inside or outside the containment.

In addition, concrete creep and shrinkage and prestress relaxation are loads to be considered in this category.

4. Abnormal Load Category

This load category covers all loads generated by the design basis accident (DBA) or loss-of-coolant accident (LOCA) and other stipulated accident conditions involving high energy pipe ruptures described above. These loads include specifically

$T_{a}$ - thermal effects and loads, generated by the DBA, including $T_{0}$ $R_{a}$ - pipe reactions from thermal conditions, generated by the DBA, including $R_{0}$

$R_{r}$ - local effects on the containment due to DBA, including $R_{r r}$ (or $Y_{r}$ ) - load generated by the reaction of a ruptured high energy pipe, including dynamic load factor (pipe whip) 
$P_{r j}$ (or $Y_{j}$ ) - load generated by jet impingment from a ruptured high energy pipe, including dynamic load factor

$R_{r m}\left(\right.$ or $\left.Y_{m}\right)$ - load resulting from impact of a ruptured high energy pipe, and other internally generated missiles (value items, bonnets, etc.)

$\mathrm{H}_{\mathrm{a}}$ - load resulting from internal post-LOCA flooding, if such an occurrence is defined in the design specification

It may be noted that for concrete reactor vessels, abnormal loads are also defined as loads which will not result in structural damage requiring repair (1980 version), while in the 1975 edition the loads were defined as those which require immediate corrective action or orderly shutdown of the plant. Also to be considered is tendon failure or material degradation equal to the total design prestress loss, e.g., $-0.15 \mathrm{~F}$.

5. Severe Environmental Load Category

Loads in this category are those to be encountered "infrequently" during the plant life, i.e., loads to occur once in a while, such $W$ - loads generated by the design wind specified for the plant site( $O B W)$

$E_{0}$ - loads generated by the operating bas is carthquake (OBE).

6. Extreme Environmental Load Category

This category is defined to contain loads to be considered "credible but highly inprobable," i.e., loads of such large recurrence periods that the probability is small that the structure will be subjected to them during its lifetime. These loads include $E_{S S}$ - loads generated by the safe shutdown earthquake (SSE) 
$W_{t}$ - tornado loading including the effects of missile impact, including

$W_{\text {tq }}$ - loads due to tornado wind pressure

$W_{t p}$ - differential pressure loads due to atmospheric pressure change

$W_{t m}$ - tornado generated missile impact effects

For either loading condition, the plant is required to respond such as to permit safe shutdown. Other loads not specifically mentioned in the code but in the USNRC Standard Review Plan includie tsunami, aircraft impact, gas explosion, probable maximum flood, etc., which are to be considered on a case-by-case basis.

\section{Failure Category}

This load category is required by the current code explicitly only for concrete reactor vessels (CRV). Failure category load combinations would normally be the result of postulated component failures of extremely remote possibility, such as $P_{m c}$ - pressurized crack condition

$\mathrm{T}_{f}$ - temperature increases in mass concrete of the CRV where concrete structural capacity is required

$Y_{j r}$ - steam impingement on prestressing anchorage assemblies $-\frac{3}{2} F$ - tendon failure or material degradation up to one-half of design basis value

and penetration closure failure. The design requirement for any loading condition of this category is that the structure shall not fail.

The above load categories are much more specific than the corresponding service level limits for metal containments. 
The Standard Review Plan [1975] contains the a complete set of structural load and resistance specifications for the purpose of insuring acceptable performance in the case of extreme environmental and/or postulated accident events. These specifications are at present structure and construction material dependent. This fact is illustrated in Table 2.1 which summarizes the current design requirements of the Standard Review Plan and other codes governing the design of reinforced concrete and steel containments and internals, as well as other category I structures. These are the ACI Standards 318 and 349, the AISC specifications, and ASME Code, Section III, Divi= sions 1 and 2. The SRP is in some cases given exceptions or additions to the provisions contained in the ACI, ASME, and AISC documents.

Despite these differences, the standards in Table 2.1 all specify safety factors in the form of allowable stress factors or resistance and load factors to insure an acceptably low risk of unsatisfactory performance. The determination of these factors relies heavily on experience in the design and construction of ordinary steel and reinforced concrete structures. To take a specific example, the design load combinations under service load conditions according to SRP 3.8.4 (ACI Standard 349) for category I reinforced concrete structures are,

$$
\begin{aligned}
& 1.4 D+1.7 L \\
& 1.4 D+1.7 L+1.7 W \\
& 1.4 D+1.7 L+1.9 E
\end{aligned}
$$


These are identical to the load factors in ACI Standard 318, with the exception that in the ACI Standard 318, the second and third combination would be multiplied by a load combination factor of 0.75 . In some cases where information on a particular load effect is limited or lacking, a load factor is assigned on the basis of the perceived similarity between the load in question and a more commonly encountered load. Thus, a load factor of 1.7 is applied to equipment live loads, normal thermal loads, and pipe reaction loads, despite the fact that (a) the character of live loads in nuclear structures may be quite different than those acting on ordinary structures, and (b) thermal effects and pipe reactions bear very little similarity to live loads.

The commentary to Section 9 of ACI Standard 349 provides some insight into the reasoning behind the selection of existing load factors. For example, higher load factors are applied to those loads for which it is felt that the probability of exceeding the design load is higher. Unit load factors are used where it is recognized that the occurence of the loads are of extremely low probability (for example, tornado loads). One of the abnormal load combinations involving the differential pressure generated by a pipe break, $P_{a}$, applies a factor of 1.25 to $P_{a}$ because of the complex nature of the consequences that could be generated by this event. Thus, the factor 1.25 chosen for $P_{a}$ is, in effect, an importance factor. For load combinations involving thermal loads which tend to be self-relieving, a 25 percent reduction in total load effect is allowed. 
There are also debatable aspects in the treatment of resistances in the various codes. For example, the resistance ( capacity reduction) factors specified in ACI Standard 349 which is primarily intended for nuclear structures, and in ACI Standard 318 intended for non-nuclear structures, are identical despite the fact that quality control and quality assurance procedures are considerably more stringent for nuclear structures.

The subjective determination of safety factors makes it virtually impossible to achieve consistent levels of reliability for the different load combinations and design situations although such consistency is imperative for nuclear structures. Standard Review Plan sections 3.8.1 through 3.8.4 require anywhere from 8 to 15 different load combinations. There is no assurance that all of these load combinations are necessary or that the load factors in these combinations are the most suitable for nuclear structures. It is conceivable that fewer load combinations may be sufficient for probabilistic design consideration and that some relative adjustment in the values of load and resistance factors is desirable. With respect to the resistance factors, the fact that corstruction and quality control practice may be sufficiently different for nuclear structures should be reflected in the selection of the resistance factors. As mentioned above it is not apparent that this has been done.

A more detailed preliminary examination of current code requirements for nuclear power plant structures is qiven in Appendix A. 


\subsection{Special Considerations for Nuclear Structures}

Al though the criteria used for the design of nuclear structures appear similar to those used for ordinary structures, as described in the previous section, there are a number of features that require special consideration:

\subsubsection{Consequence of Failure}

Perhaps the most significant consideration is the relation between the design criteria and the consequences of failure. The failure of nuclear structures, functional or structural, may ultimately lead to the release of radioactivity, a consequence of the gravest societal impact. Thus, the risk of-such an accident must be minimized by assuring a very low probability of its occurrence. Therefore, the level of structural reliability implied by specific design criteria for nuclear structures must be much higher than that for ordinary structures. An acceptable level of occurrence frequency for such an accident is difficult to establish, however, although increasing efforts are being made to quantify such an acceptable level as exemplified by the recent report entitled "An Approach to Quantitative Safety Goals for Nuclear Plants" by the Advisory Committee on Reactor Safeguards, October 1980. Once an acceptable level of risk is quantified, say, in terms of a probability, further work is necessary to allocate this probability among the appropriate structural elements comprising category I structures. Indeed, it is this allocated probability that must be associated with the load combinations to be considered for the design of each structural element. 


\subsubsection{Loading Conditions Unique to Nuclear Structures}

The loading criteria for the design of ordinary buildings tend to be evolutionary in nature and rely, to a considerable extent on past experience. Since past experience is generally deemed an acceptable indication by the profession, the reliability levels for ordinary buildings associated with existing criteria are, on the whole, appropriate and this established a reference reliability level. Some effort for probabilistic combination of dynamic loads acting on nuclear structures and pipings has been made recently [Reich, et a7., 1980]. However, experience with load criteria for nuclear structures is rather limited so that a similar interpretation cannot be made with confidence at this time. A closely related problem arises from the number of loads that need to be considered for nuclear structures. Many of these loads are either not germane to or have not been considered for ordinary structures. As a result, there are virtually no data available on these loads on the basis of which suitable values for their design magnitudes can be specified. This lack of data also creates the difficulty that the specifications for nuclear structures cannot be linked to experience at least at present.

\subsubsection{Postulated Accidents}

Accident loads are uniquely defined by the NRC (Standard Review Plan, NUREG-75/087) and by the Nuclear Industry (for example see NED0-21061, Sept. 1975) as far as nuclear category I structures are concerned. Among the more significant accidents specified for design evaluation are the so-called Loss-of-Coolant accidents (LOCA). These accidents results from a sudden break of a high pressure pipe attached to the Nuclear Steam Supply System (NSSS). According to the size of the break, three types of LOCA's are specified; (1) Design Basis Accident (DBA), (2) Intermediate Break Accident (IBA) and (3) Small Break Accident (SBA). 
The most significant of the above three accidents is the DBA which give rise to pool swell, condensation oscillation and chugging phenomena. All of these postulated events are quite complex as illustrated by the typical Mark I (BWR) DBA events following a recirculating pipe break which is described below:

Due to the break which can be assumed to occur at the welded end of a nozzle attached to the reactor pressure vessel, high pressure steam is ejected into the annulur space between the reactor vessel and the bioshieid wall. The vessel and wall must be designed for this pressure. This is called annulus pressurization. After the steam leaks into the drywell space and builds up pressure, the high pressure air is pressed into the suppression chamber through the vent pipes to the ring header and blown into the pool through the downcomers. The high pressure air first forces water "legs" in the downcomers out of the pipes. This causes water jet forces which only result in an insignificant load. Following this, however, the pressurized air enters into pool and forms growing bubbles which not only cause hydrodynamic pressure to act on the torus, but also purge the water upward. The air in the upper portion of the torus is compressed causing an upward pressure on the torus due to the rising of water. The rising water impacts the vent header, vent lines, and walkways. The continuing rising water finally breaks up and turns into a froth mode. The structures also are subjected to froth impact but at a lower intensity. Besides the impact loads, structures being submerged in the rising water are subjected to drag loads. 
After all drywell air is purged into the torus free space, the steam-air mixture starts entering into the pool. The continuing influx of steam flow and condensation causes near steady state oscillation. The condensation lasts from one to five minutes. Following this the steam influx rate is reduced and the condensation process degenerates into intermittent pressure purges. This is the last event of the DBA LOCA and is called "chugging". Needless to say, the LOCA loads are different for different reactors. Thus, the PWR as well as other General Electric reactors, such as the Mark II and Mark III reactors will have LOCA's with different forcing functions. The statistical distribution of these loadings appears to be lacking and a field survey (i.e., consensus estimation as described in Appendix B) together with an intelligent estimate is at present required. Furthermore, the postulated large pipe break accident (DBA) is considered to be a once in the life time (40 years) event. Whether it needs to be combined with earthquake, and/or other time dependent events such as SRV, etc., requires further evaluation.

Other accident conditions caused by the LOCA events include: (1) Accident pressure rise $\left(P_{a}\right),(2)$ Accident pipe reactions, (3) Accident temperature rise $\left(T_{a}\right),(4)$ Accident whipping action pipe of rupture restraints $\left(Y_{n}\right),(5)$ Accident jet impingements $\left(Y_{j}\right),(6)$ Accident internal missiles $\left(Y_{m}\right)$, (such as valve items, bonnets etc.), and various (7) Postaccident or post-LOCA events

The above condition constitutes the most important postulated accidents. As can be seen, these are unique to the nuclear structure and thus, as mentioned, these structures require special considerations. 


\subsubsection{Complexity and Difficulty in Decomposing the structural system.}

A major difficulty in performing a reliability analysis for a structural system is the inherent complexity of the system. Such complexity levels are also common in electronic and mechanical systems which have been successfully analyzed using a variety of techniques.

Techniques such as fault trees, event trees, and reliability block diagrams all decompose the system into smaller subsystems and components which can be more easily analyzed and combined to yield a system reliability expression.

It is difficult to decompose the structural systems because of the strong degree of coupling between the structural subsystems and elements. Thus, we must search for existing or new decompnsition methods which allow decomposition in a manner compatible with the physical problem.

The conventional methods of reliability analysis, the difficulties in applying them to structural systems, and the means of circumventing these difficulties are discussed in Sec. 3.4.

\subsubsection{The Design Process and Apportionment of Subsystem Reliabilities.}

In the preceding paragraphs we spoke of reliability analysis techniques. We are actually most interested in reliability design techniques. The most desirable reliability design is that of synthesis.

To perform synthesis we begin with the specifications and a mathematical procedure for designing the system. We test to see that a solution is possible and utilize the procedure to determine a detailed design which meets the specifications. Unfortunately this ideal method is seldom feasible in any branch of engineering (the one notahle exception is the field of electric network synthesis). 
In practice, design is an iterative trial and error procedure. A feasible structural design is proposed, the parameters are determined, and the reliability of the structure is analyzed. If the reliability is too low revisions are proposed to strengthen the structure and iteration continues until a design is achieved which meets the target reliability. Similarly, if upon initial analysis one finds that the reliability of the structure significantly exceeds the goal, one can accept the design and deliver "extra quality" to the customer. Another alternative is to lower the reliability toward the goal level by decreasing the cost (and strength) of certain features of the design.

As an aid in achieving an acceptable trial and error design, it is common to apportion the overall reliability goal among the various subsystems. For example, suppose that there are three independent subsystems all of which must succeed for the system to succeed. If the system reliability goal, $R_{S}$, was 0.99 then if each of the sub-systems were to have the same reliability goal, $R_{S S}$, then

$$
\begin{aligned}
& R_{S}=R_{S S}{ }^{3}=0.99 \\
& R_{S S}=\left(R_{S}\right)^{1 / 3}=(0.99)^{1 / 3}=0.9967
\end{aligned}
$$


Apportinnment is a virtual necessity if each subsystem is to be constructed by a different contractor, since we must set independent goals for each member of the team. Apportionment also depends on being able to decompose a system, which is discussed further in Sec. 3.4. 
Table 2.1. Current Design Practice for Nuclear Structures

\begin{tabular}{|c|c|c|}
\hline \multirow[b]{2}{*}{ Material } & \multicolumn{2}{|c|}{ Structure Type } \\
\hline & $\begin{array}{l}\text { Containments and } \\
\text { Internal structures }\end{array}$ & $\begin{array}{l}\text { Other } \\
\text { Category I Structures }\end{array}$ \\
\hline $\begin{array}{l}\text { Reinforced } \\
\text { concrete }\end{array}$ & $\begin{array}{l}\text { SRP } 3.8 .1 \text { and } 3.8 .3 \\
\text { ASME Section III, Division } 2 \\
\text { (ACI Standard } 359 \text { ) } \\
\text { A1 lowable stress, Dut certain } \\
\text { loads are factored for combi- } \\
\text { nations involving severe, } \\
\text { extreme and abnormal loads. } \\
\text { ACI Standard } 318 \\
\text { Strength design }\end{array}$ & $\begin{array}{l}\text { SRP } 3.8 .4 \\
\text { ACI Standard } 318 \\
\text { Strength design } \\
\text { ACI Standard } 349 \\
\text { Strength design }\end{array}$ \\
\hline Steel & $\begin{array}{l}\text { SRP } 3.8 .2 \text { and } 3.8 .3 \\
\text { ASME Section III, Division } 1 \text {, } \\
\text { Subsections NE and NF } \\
\text { Allowable stress design } \\
\text { AISC Specifications, Parts } \\
\begin{array}{l}1 \text { and } 2 \\
\text { Part } 1 \text { - allowable stress } \\
\text { Part } 2 \text { - plastign design }\end{array}\end{array}$ & $\begin{array}{l}\text { SRP } 3.8 .4 \\
\begin{array}{l}\text { AISC Specifications } \\
\text { Parts } 1 \text { and } 2 \\
\text { Part } 1 \text { - allowable } \\
\text { stress } \\
\text { design } \\
\text { Part } 2 \text { - plastic }\end{array}\end{array}$ \\
\hline
\end{tabular}


. 
of the approximations enabling each load effect to be treated as a random variable may be used to determine the load and resistance factors.

8. The observation made in (7) above indicates the necessity for developing a method more directly based on the limit state probability, by which the load and resistance factors can be determined when two or more component load effects are randomly time-varying. In this connection, the methods that are currently available for evaluating the limit state probability in approximation for a structure under multiple time-varying loads are reviewed and some of these are found to be useful. However, further efforts are definitely needed to improve these methods so that not only the accuracy of the approximation can be upgraded but also one can deal with more realistic analytical models of the load effects involved. 


\section{PROBABILITY-BASED DESIGN FOR NUCLEAR STRUCTURES}

\subsection{Introduction}

The previous discussion has focused on the need to specify safety factors in order to account for design uncertainties. In addition, some questions in specifying subjectively selected safety factors based solely on practical experience have been mentioned. It may be observed that while strength and load parameters are nondeterministic, they nevertheless exhibit statistical regularity. This fact suggests that probability theory should furnish the framework for setting specific limits of acceptable performance for design. The overall objective of this study is to develop the structural design criteria based on the required levels of reliability for safety and performance evaluation of nuclear structures.

The idea that dispersion (or statistical variation) in a parameter such as yield stress or load should be considered in specifying design values is not new, and many standards indeed have used this concept. Rather than requiring that the risk arising from these uncertainties be eliminated entirely, a goal which may not be attainable or may be prohibitive in cost, the risk can be managed by reducing it to some acceptably small level. Probability theory and reliability-based design provide the framework for developing design criteria which insure that the probability of unfavorable performance is acceptably small. Specifically, probability theory and structural reliability methods make it possible to select safety factors that will be consistent with a desired level of reliability (acceptably low probability of unsatisfactory performance). 
The development of reliability-based criteria for design of nuclear structures has four basic tasks:

1. Develop necessary data base to determine occurrence frequencies and intensities of various environmental and postulated loads and resulting structural responses. (See Section 3.2)

2. Establish models to describe the behavior of the structural system under prescribed design conditions. (See Section 3.3)

3. Establish procedures for computing reliability of elements, different elements. components and of the structural system. (See Section 3.4)

4. Specify safety checking procedures to be used by the structural designer. (See Section 3.5).

Each of these tasks shares features in common with reliability based criteria for ordinary structures. However, there are also certain features that are either unique to nuclear structures or that have not been important enough for ordinary structures to warrant extensive attention or research.

In the remainder of Section 3, existing analytical studies and experimental data are reviewed with regard to their specific applicability to the above four basic tasks of probability-based design of nuclear structures. The twofold purpose of this review is to (1) highlight existing research upon which standards for the design of ordinary structures are based and which appears to be immediately applicable for nuclear structures, and (2) point out problem areas where additional research appears to be necessary at later stages of the program. 


\subsection{Basic Data}

\subsubsection{Structural Loads}

\subsubsection{General Discussion Structural loads are idealized concentrated} or distributed forces (or imposed deformations) that result from the operating environment of the structure. The evaluation of the structural loads has two important aspects: development of probabilistic load models and determination of the statistics loads and load effects of a structure.

It is useful to think of a structural load effect, $Q_{i}$ (beam moment, column shear, etc,) as being determined from a basic structural load parameter $A_{j}$, a load modeling parameter $B_{i}$ and a structural analysis parameter, $c_{i}$.

$$
Q_{i}=C_{i} B_{i} A_{i}
$$

where $A_{i}$ is determined by the environment in which the structure functions and is essentially independent of the structure. $A_{j}$ varies randomly in space and in time and statistical characteristics of $A_{j}$ may be found from load surveys, in situ pressure measurements, etc. $B_{i}$ is a modeling parameter that transforms the spatially and temporally varying load into an equivalent uniform or concentrated (and often static) load for purposes of design. $B_{i}$ may depend on certain gross structural characteristics, e.g., frequency and damping in dynamic problems. $C_{i}$ is an analysis factor that transforms load product $A_{\mathbf{i}} B_{\mathbf{i}}$ into a load effect (moment, shear). Uncertainties in $\mathbf{C}_{\mathbf{i}}$ arise from such factors as two-dimensional or axisymmetric idealizations of three-dimensional structures, rigidity of connections, etc.

The remainder of this section on loads is concerned primarily with the availability of data to statistically characterize $A_{i}$ and $B_{i}$ in Eq. 3.1. The discussion of "Models of System Behavior" in Section 3.3 is relevant to the determination of statistics of $C_{i}$. 


\subsubsection{Dead Load}

Under normal operating conditions, the effect of dead load from the weight of the structural system itself and the weight of permanent equipment and other installations such as piping, ductwork and cable trays may be a significant part of the total load effect. The probability distribution for dead load appears to be normal. Variability in dead load arises from variations in the unit weights of construction materials, and especially for concrete structures, variations in component dimensions from those specified on working drawings. Additional uncertainties may arise from permanent items that are added to the structural system during the service life without the knowledge or approval of a designer. Forces due to prestressing are in most cases considered as dead load.

Data on the variations in unit weights are generally available for steel and concrete. Information on variabilities in dimensions of beams, columns, and slabs is available for conventional reinforced concrete structures. However, since quality control on a nuclear structure site may be more stringent, additional field surveys should be conducted to establish whether the dimensional variabilities for these structures are less pronounced. Inspection or consensus estimation survey with regards to operating facilities could heip in determining whether there is a tendency to add unplanned permanent items over a period of time, as there is in conventional buildings.

Two interesting observations have been drawn in previous studies [Bresler, 1974; Ellingwood, et a1., 1980] regarding dead load in conventional structures. First, there is a tendency for most structural designers to underestimate the actual dead loads, typically by 3 to 5 percent. Apparently, there are a number of seemingly inconsequential items that are usually ignored but tend to add up. Second, while the variability in dead load is by no means negligible, it does not seem to be high enough to justify the load factor of 
1.4 specified for combinations with time-varying loads for design of concrete structures. A more suitabie value may be somehwhere between 1.0 and 1.2. The implication is similar for design of steel structure. These observations are of considerable importance in design of massive structures.

Loads arising from naturally occurring meteorological or geologic phenomena include the effects of wind, snow, ice, rain, earthquake and soil movement. In principle, the characterization of these loads from a statistical point of view should be no different for nuclear structures than for ordinary structures. A significant amount of data have already been collected and analyzed in order to develop loading provisions for ordinary buildings [ANS A58.1 - 1972; Ellingwood, et al., 1980]. While these data and their analys is will be considered at some length in a forthcoming report, a brief summary of the present state of the art is provided below:

\subsubsection{Wind Load}

The wind pressure used in the design of buildings according to ANSI Standard $A 58$ is given by,

$$
w=c K_{z} G C_{p} v^{2}
$$

in which $c=$ constant, $K_{z}=$ exposure coefficient that depends on location and elevation, $C_{p}=$ pressure coefficient, $G=$ gust factor and $V=$ wind speed referenced to a height of $10 \mathrm{~m}$ in open terrain. $C_{p}$ depends on the general geometry of the structure (ratio of height to width, rectangular shape) and $G$ depends on the turbulence of the wind and the dynamic interaction between the structure and the wind [Simiu, 1978]. The term $c K_{z} v^{2}$ is analogous to $A_{f}$ in Eq. 3.1 since it is structure-independent; $G C_{p}$ is analogous to $B_{i}$ because it serves to average out local pressure fluctuations over large areas.

For ordinary buildings, $V$ corresponds to a 50 year mean recurrence interval (MRI) annual extreme fastest mile wind speed [ANS A58, 1972]; 
equivalently, the probability of exceeding the design wind speed is $0.02 /$ year. The A58 Standard provides maps for determining $V$ in the United States. These have been constructed by fitting extreme value probability distributions to wind speed data gathered at over one hundred airport measuring stations, calculating the 50 year MRI values from the probability plots, and constructing a contour map of 50 year MRI wind speeds consistent with the observations.

For nuclear structures, the 100 year MRI value from the same wind speed probability distribution is specified instead, corresponding to an exceedance probability of $0.01 /$ year. Thus, a problem in wind speed analysis is in deciding on an appropriate exceedance probability upon which to base the specified nominal design wind speed. The data gathered in connection with efforts to develop probability based loading criteria for ordinary structures should be applicable here also [Simiu, 1979; Batts, 1979].

The coefficient $k_{z}$ describes the wind profile with height and depends on the surface roughness in the earth's boundary layer. Both logarithmic and exponential relations have been proposed to describe the increase in wind speed with height [Simiu, 1978], and experimental data are available to define the constants in these models. $G$ and $C_{p}$ (or frequently the product $G_{p}$ ) have been determined for a variety of ordinary structures through wind tunnel modeling, in situ pressure measurements, and dynamic analysis. Much of this information should be adaptable, with some modifications, to nuclear structures.

\subsubsection{Tornado Load}

Much of the research on tornado effects conducted over the past decade has beendirected toward providing criteria for design of nuclear structures. Tornadoes are characterized by their rate of occurrence, direction, intensity, path width and length. Intensity, length and width are commonly measured 
by the Fujita F-scale and Pearson length and width scales, (FFP), respectively. Tornado effects considered in the design of nuclear structures fall into three categories: (1) Velocity-related pressure; (2) Pressure differential or drop, and (3) Effects of tornado-borne missiles. None of these effects is currently considered in the design of ordinary buildings and structures [ANS A58, 1972].

Since tornado occurrances are site dependent, the probability of tornado wind at a particular location is obtained from the occurrence rate of tornadoes at the site and from the probability distribution of intensity or maximum wind velocity. It is to be noted that tornado data have been systematically collected since 1971 [Abbey, 1976; Twisdale, 1978]. The (complementary) probability distribution of tornado wind speed $V$ is given by:

$$
P[V>V]=P[V>V \mid S] \cdot P[S]
$$

where $P[V>V \mid S]=$ probability of event $V>V$ on the condition that a strike occurs, and $\mathrm{P}[\mathrm{S}]=$ probability of a strike. All recent tornado risk analyses start from this basic relationship, however, there are differences in the way the terms in Eq. 3.3 are evaluated. A brief description of some aspects of the methodology is given below. For more comprehensive discussion see [Abbey, 1976].

The strike probability $P[S]$ is given in terms of a map of the United States in which the annual probability of any tornado striking a site is given for $1^{0} \times 1^{0}$ (or $5^{0} \times 5^{0}$ ) squares or in terms of contours of equal probability. In early papers [e.g., Thom, 1963], the strike probability was calculated as,

$$
P[S]=\nu \frac{\bar{a}}{A_{0}}
$$

in which $v=$ mean rate of tornado occurrence, $\bar{a}=$ mean tornado path area, and $A_{0}=$ grid area $\left(e . g .\right.$, a $1^{0} \times 1^{0}$ grid): Parameters $v$ and $\bar{a}$ were estimated from historical records. 
The current NRC regulations are based on the same analytical procedure with an expanded data base [Markee and Beckerley, 1974]. Specifically, a zone (or contour) map of "equiprobability" design basis tornado wind speeds $V_{T}$ can be constructed from the relation,

$$
P\left[V>V_{T}\right]=10^{-7} / P[S]
$$

in which the accepted annual probability of exceeding the design basis tornado $V_{T}$ is $10^{-7}$ per year. Furthermore, it is assumed that $P[V>V \mid S]=P[V>V]$ (i.e., the distribution of wind speed is independent of strike probability), the value of $P[S]$ is determined from Eq. 3.4, and the velocity $V$ is assumed to have a lognormal probability distribution. The correlation between $V$ and F-scale given by Abbey and Fujita [1975] is utilized to convert historical tornado intensity data that is reported in terms of F-scale to equivalent velocity values. This approach leads to estimates of $V_{T}$ in excess of $350 \mathrm{mph}$ in most of the United States east of the Rocky Mountains.

The assumption that the events "strike" and " $V>V$ " are statistically independent is not borne out by a closer examination of the data. The tornado intensity and maximum wind speed are positively correlated to the path area of the tornado. Moreover, the approach described above assumes that the peak velocity occurs over the entire path area. The dependence between path area and maximum velocity is taken into account in the model of Wen and Chu [1973]. Garson, et al. [1975] extended Wen and Chu's model to include the effect of structures of finite size relative to the tornado path area. This requires an assumption on the variation of wind speed with distance from the center of the vortex. Twisdale [1978] has developed a procedure to account for observation errors. Abbey and Fujita [1975] have considered variations in intensity 
along the length of the tornado path. The incorporation of these refinements reduces the values of the design velocities corresponding to an annual exceedance probability of $10^{-7}$ by approximately 15 percent when compared to those reported in the earlier studies [Markee and Beckerley, 1974].

It appears that none of these models completely incorporates al1 aspects known to affect the tornado loads. A substantial effort devoted to synthesizing these models is currently underway.

The wind-induced pressure due to the tornado may be computed from the design velocity by the expression

$$
W_{T}=\frac{1}{2} \rho C_{P} V_{T}^{2}
$$

where $\rho$ is the mass density of air. Comparing this equation with Eq. 3.2, it is seen that the terms $G^{-}$and $K_{z}$ are assumed to be equal to unity. It is assumed that $V_{T}$ includes any necessary amplification for turbulence intensity because the tornado wind velocities usually are inferred from observed damage rather than measured directly. It is usual to disregard the slight tendency for tornado wind speeds to increase with height. This should not lead to any significant error for nuclear structures, most of which are under $75 \mathrm{~m}$ in height. The dynamic characteristics of such structures are such that the pressure can be treated as an equivalent static load.

A second tornado related effect arises from the atmospheric pressure change that occurs if the tornado path overlaps a portion of the structure. This is caused by a pressure gradient within the vortex that leads to pressures much lower that atmospheric within the vortex, Using the equation for cyclostrophic wind [Simiu, 1978], this pressure gradient is related to the tangential wind speed by, 


$$
\frac{d p}{d t}=\rho v_{t}^{2} / r
$$

in which $r$ is measured from the center of the vortex and $v_{t}$ is the maximum tangential wind velocity. The maximum pressure drop, $p_{\max }$, may be as high as 3 psi in regions subject to tornadoes of extreme intensity.

If the structure over which the tornado passes is completely unvented, the outward pressure on the exterior structural elements will reach $p_{\max }$. At the other extreme, for structures that are completely vented, pressures on interior and exterior surfaces will equalize. Thus, the load effects due to the pressure change depend on the configuration of the structure and the possibilities for flow of air between compartments within the building. In effect, this is a postulated load condition and at present it is not clear how such postulated loads should be treated from a statistical point of view.

A third tornado effect is the impact of various missiles that may be picked up and transported by the vortex. An estimation of these effects requires a set of assumptions regarding the different potential types of missiles, their location with reference to the site, their aerodynamic characteristics, and the wind velocity field [Simiu, 1978]. At the present time this analysis is handled by requiring the postulation of a specific scenario. It is assumed that missiles are injected into the wind model at the point of maximum velocity and that they impact the structure end-on. Obviously this is a combination of worst-case conditions.

The probability distributions of tornado wind load effects will be site-dependent. It will be necessary to either use site-averaged parameters or to choose a manageably small number of representative (generic) sites for which reliability analyses will be performed in subsequent phases of this program. 


\subsubsection{Snow Load}

The roof snow load for ordinary structures in the A58 Standard is calculated on the basis of the 50 year MRI ground snow load q multiplied by a coefficient $c_{s}$ that transforms the ground load to an equivalent roof load. In equation form,

$$
S=c_{s} \cdot q
$$

The ground snow $q$ is independent of structure and is equivalent to the term $A_{i}$ in Eq. 3.1. The factor $c_{s}$ depends on building exposure, roof geometry, thermal characteristics, and is analogous to $B_{i}$ in Eq. 3.1. Statistical characteristics of $q$ can be determined from observations of snow depths and water equivalent weights. These can be obtained from the National Weather Service and/or the Soit Conservation Service. These data form the basis for the snow maps for various MRI given in the A58 Standard. The factor $c_{s}$ has been obtained for common building geometries by measuring snow accumulation in situ [ANS A58, 1972] and more recently by model tests in wind tunnels and water flumes. A program is underway to collect similar data for nuclear power plants in the Northeast quadrant of the United States.

The ground snow load used for nuclear structures under normal operating conditions corresponds to the 100 year mean recurrence interval load, This 100 year MRI load is surcharged (apparently arbitrarily) by the load from the maximum probable 48 hour precipitation for checking against extreme environmental conditions. This latter combination is roughly equivalent to a load with a mean recurrence interval of $10^{6}$ to $10^{7}$ years. There is a question as to whether this specification is unecessarily conservative. 


\subsubsection{Earthquake Loads}

Aseismic design requires a characterization of the intensity of ground motion at the plant site (commonly, peak ground acceleration; but peak velocity may also be appropriate in certain circumstances), the determination of an appropriate design spectrum or time histories of ground motion corresponding to the selected design intensity, and finally the computation of the maximum structural response. It is convenient to consider earthquake loadings on structures as the product of several factors:

$$
E=K S S_{A} A W
$$

in which $W=$ weight of the structure or component, $A=$ ground acceleration (in unit of g), $S_{A}=$ spectral amplification factor which depends on general structural characteristics such as modal frequencies and damping, $S=$ soil-structure interaction factor, and $K=$ system ductility factor. Eq. 3.8 describes an equivalent lateral static force for simple structures where the first mode response dominates. In complex systems, where the dynamic response is significantly affected by the higher modes, $\mathrm{E}$ in Eq. 3.8 may be interpreted as a modal force. The total peak response is then obtained by combining the peak modal responses.

It is important to note, that there are significant uncertainties associated with each of the variables in Eq. 3.8. For example, uncertainties with respect to $A$ (or any other peak ground motion parameter) arise from: (1) randomness in the source-generating mechanisms, (2) estimation of future events at sources where the site may affect seismicity, and (3) attenuation 
of ground motion from the source to the site. Furthermore, uncertainties in modal frequencies, damping, and post-elastic system structural behavior give rise to variabilities in $S_{A}$ and $K$.

The basic earthquake intensity for the design of nuclear structures is specified by two levels of ground motion corresponding to an "operating basis earthquake" (OBE) $E_{0}$ and a "safe shutdown earthquake" (SSE), $E_{S}$. Current regulations [10 CFR 100] define the OBE to have an intensity no less than one-half the intensity of the SSE. It should be noted that as the SSE is a postulated maximum credible event and is not determined from a statistical analysis. Similarly, the OBE may not ąiways be statistically based. The probability of exceeding the OBE and SSE can sometimes be estimated from historical data. Although data on ground accelerations at a site tend to be speculative, it has been suggested that $E_{0}$ corresponds to an annual probability of exceedance of 0.002-0.01 (MRI $=100-500$ years) while $E_{S}$ corresponds to an annual exceedance probability of $10^{-3}$ to $10^{-4}$ (MRI $=$ 1,000 - 10,000 years). Note the lack of consistency in MRI with the tornado event and extreme snow load which is assumed to be approximately $10^{7}$ years.

In a recent study [A]germissen and Perkins, 1976], an attempt was made to construct earthquake hazard maps for the U.S. based on constant probability of exceedance of gournd motion intensity for the entire country (risk maps in current building standards tend to be based on the maximum historical event, which leads to inconsistencies in risk from site to site). Many engineers concerned with seismic design of nuclear structures feel that the OBE should be similarly characterized. If this were done, it is likely that the ratio of OBE to SSE would be considerably less than one-half in the eastern part of the U.S., while in certain areas west of the Rocky Mountains, it may be greater than 
one-half [Howard, 1976]. Such a specification would also affect the load factors and load combinations.

In current NRC regulations, the effect of randomness in earthquake ground motion on structural responses is treated by specifying the response spectra developed by Newmark, et a1., [1973] and Hall, et al., [1976]. These response spectra are determined from the statistical analysis of the structural responses to actual earthquake records. The maximum responses computed for various frequencies and damping ratios are normalized to suitable peak ground motion parameters. In effect, then, the spectral amplification $\mathrm{S}_{A}$ in Eq. 3.8 is treated as a random variable, with a mean and standard deviation that depend on frequency and damping. A design spectrum is then obtained by computing the meanplus-one-standard-deviation level of $\mathrm{S}_{A}$ for each frequency and damping ratio in the velocity-amplified and acceleration-amplified regions of the response spectrum.

Differences in response due to source mechanism, intensity of ground motion, attenuation, and soil conditions at the site are not considered explicitly in computing the statistics of $S_{A}$ from recorded ground motions. Thus, there is a presumption in the above approach that the statistics of $S_{A}$ are not sensitive to these factors. More recently, Wang [1979] and Seed [1979] [1976] took site soil conditions into consideration. McGuire [1974] also attempted to separate out these factors by performing a regression analysis of $\mathrm{S}_{A}$ on measures of intensity and attenuation. This analysis pointed out some inconsistencies in probability levels for the procedure previously described. None of these analyses take into account the uncertainties in modal frequency, damping, or in the behavior of the systems near ultimate conditions. Some of these uncertainties may be substantial.

An alternate procedure is based on random vibration theory [VanMarcke, 1976]. This method assumes that the excitation and structural response are 
stationary, (at least for the duration of strong moition shaking), that the excitation power spectral density can be established and that the response statistics of interest for the subsequent reliability analyses and load combination studies (e.g., variance, peak distribution, mean up-crossing rates, as discussed later in Section 4) can be calculated. The stationarity assumption is reasonable for nuclear structures because the periods of vibration for these structures which range from 0.03 to $2 \mathrm{sec}$ are considerabiy shorter than the duration of strong motion shaking which usually lasts for 10 to $30 \mathrm{sec}$. The determination of an appropriate excitation spectral density for a particular site may present a problem. However, some procedures for relating the spectarl density to the response spectrum have been suggested [Udwadia and Trifunac, 1974]. These methods appear to be applicable to different site conditions. This approach for determining earthquake response statistics will be further pursued because it offers a number of advantages for reliability anlayses and load combination studies.

\subsubsection{Limitations of Environmental Load Data.}

Data from which design loads for meteorological phenomena are based, typically represent records of 20 to 40 years duration [Ellingwood, et a1., 1980]. The confidence interval on the 50 year MRI value estimated from samples of this size is rather smal1. Indeed, it may be shown that the mode of the maximum of $m$ observations is approximately equal to the m-year MRI value. In the case of earthquakes, additional historical data are available, dating back perhaps 200 years in the United States however, the usefulness of early less-quantitative data for providing magnitude and intensity values or for deriving probability distributions for ground acceleration is uncertain. Moreover, the extreme loads for which nuclear structures are designed are rarely observed in practice, and thus their specification requires an extrapolation of the probability distributions far beyond the range of observed data. 
While the selection of specified load values corresponding to mean recurrence intervals of 50 - 100 years usually is not especially sensitive for the assumed probability distributions, this may not be the case for nuclear structures because of the additional-conservatism (MRI greater than 1,000 years, in some instances) with which they are sejected. This suggests that the probability distributions for loads should be subjected to careful statistical tests and be examined for their consistency with the physics of the load generation and transfer processes, before being accepted for reliability analysis and probabilistic design of nuclear structures. In many instances this has not been done in reliability studies involving ordinary structures, where the probability distributions tend to be selected for mathematical expedience.

\subsubsection{Live Loads}

Live loads arise from the operating conditions of the power plant. Some of the live loads caused by routine maintenance, from cranes, and from general purpose heavy equipment, would be similar to those found in nonnuclear power plants or in other heavy industriàt applications. However, load survey data with which to define the spatial and temporal variabilities in these loads are not available. Moreover, probabilistic load models [Peir and Cornell, 1973] that have been developed for ordinary live loads have not been extended to industrial applications. Design live loads specified by different design firms vary widely [Rafay, 1980] for power plants.

Certain live loads, such as those arising from the refueling operation would be unique for nuclear structures. In contrast to many of the live loads above, these might be expected to be quite predictable due to the stringent controls exercised and the lack of variation in procedure. Many power plant designers seem to feel that these special loads are essentially 
- deterministic. If this is irrleed the case, the load factor for these loads should be unity, rather than the 1.7 value required in Section 3.8 .4 of the current Standard Review Plan. Additional data needs to be collected, however, in order to confirm or deny this speculation. It is forseen that available probabilistic load models will need to be extended and that surveys will have to be conducted to determine suitable live load statistics.

Loads that result from thermal effects during normal operating and shutdown conditions and from pipe reactions could be obtained by in-service monitoring of nuclear structures during normal operating conditions over an extended period of time. The analysis of these data has yet to be performed but should not present any particular problem.

\subsubsection{SRV Discharge Loads}

Another type of load that requires further investigation involves probabilistic load models pertaining to transients generated by safety relief valves. The transients result in pressure pulses that act on the primary system of the reactor. In BWR plants these valves are attached to the main steam lines and are installed at various locations inside the drywell structure. Downstream piping systems connect the SRV's with the suppression pool of the plant.

During the operation of the plant several safety relief valves may actuate either automatically according to the valve set points or manually through external signals. When an SRV actuation occurs, the steam released from the primary system is transmitted into the suppression pool where it is condensed. Due to the SRV discharge the pool experiences oscillatory distrubances which result in dynamic pressures on its boundaries. The containment and drywell structures must be able to withstand these pressures.

The responses of these structures due to SRV loads are very significant especially when they are combined with other responses such as earthquake loads. 
A typical plant could contain up to 20 valves, which could be actuated in a random manner. Peak values, frequency distribution and disturbance arrival time at the discharge device are random variables which need further investigation.

\subsubsection{Abnormal Loads}

In addition to the aforementioned basic loads that arise from the natural environment or from the normal operating conditions of the structure the occurrence of certain loads is dependent on whether one of the basic loads exceeds a threshold value. Examples of these conditional or initiated loads would be pressurization or pipe break loads caused by earthquake damage to a component of the plant. These are associated with the postulated accidents described in Section 2.3. As pointed out in that section, these loads depend in part on the physical characteristics of the plant. Such conditional loads are only rearely considered in the design of conventional structures. There is little statistical data available with which to characterize such loads and there is no experience in implementing such loads in design standards, other than the specification of the abnormal load combinations in the Standard Review Plans. It remains an open question as to how effects of these loads should be combined with normal loads in structural design.

Table 3.1 summarizes the previous discussions regarding the applicability of existing dead, live, environmental, and operational load data for reliability analysis of nuclear structures. 
There are numerous additional sources of loads that, under certain circumstances, must be considered in the design of nuclear structure. These would include: airplane impacts; explosions from nearby transportation of hazardous substances; tsunami and/or hurricane storm surges for plants located near the ocenas and flood loadings for plants situated in river flood plains. While there are some statistical data on the occurrence and relative magnitude of these events (e.g., 200-year mean recurrence interval floods), the conversion of the data to meaningful structural load effects is complex. The load effects from these phenomena are not covered explicitly in the matrix of design load combinations defined in the Standard Review Plan. Instead, they are covered as special cases, where appropriate. Accordingly, no attempt will be made in this research program to define the statistical characteristics of these load effects. 


\subsubsection{Structural Resistance}

\subsubsection{General Discussion}

The resistance, or load carrying capacity, of structural members and components is usually expressed by an analytical formula which is based on principles of structural mechanics and has been verified by laboratory and/or full-scale testing. It has been recognized that the variables affecting structural capacity are statistical in nature. Consequently, over the past decade, there has been a considerable impetus to statistically characterize such basic variables as the material strengths, the section properties, etc. as well as the structural resistances of the members themseives.

In this section, we begin with a general discussion of the analysis of statistics of resistance. This is followed by a brief review of what currently is available for steel and reinforced concrete structural elements, and a discussion of data needed for the reliability analysis of nuclear structures.

The structural resistance is given by

$$
R=R\left(x_{1}, x_{2}, \ldots, x_{k}\right)
$$

in which $x_{1}, x_{2}, \ldots, x_{k}=$ basic variables which would include material properties (e.g., yield or ultimate stress of steel, crushing strength of concrete), and structural dimensions or section properties, and $R()$ is based on principles of structural mechanics and experimental data.

The basic variables are random and contribute to the uncertainty in $R$. Their statistics may be determined through strength of material tests and measurements of structural elements. These data are gathered under carefully 
controlled conditions. It should be emphasized that the statistics of the basic variables used in reliability analysis should be representative of the values expected insitu and, accordingly, would depend on quality assurance and control procedures followed during construction. Any dependance of material properties on rate of loading should also be considered.

Additional uncertainties in $R$ would arise from the analytical modeling of the structural element or system. Such models invariably contain idealizations (fixity and/or continuity at joints, neglect of strain hardening, neglect of three-dimensional effects, for example). Uncertainties in this modeling process contribute to uncertainties in $R$. If,

$$
R_{m}=R_{m}\left(x_{1}, x_{2}, \ldots, x_{j}\right)
$$

in which $R_{m}=$ modeled resistance as a function of the basic variables $x_{j}$ and in which $j \leqslant k$ (Eq. 3.9), in general, because of the simplifying assumptions in the moder, the true resistance $R$ is,

$$
R=B R_{m}
$$

in which $B=$ factor to describe the effect of modeling. Assuming $B$ and $R_{m}$ to be uncorrelated, the mean and coefficient of variation in $R$ become,

$$
\begin{aligned}
& m_{R}=m_{B} m_{R_{m}} \\
& v_{R}=\left[v_{B}^{2}+v_{R_{m}}^{2}\right]^{1 / 2}
\end{aligned}
$$

in which $m_{B}, V_{B}=$ mean bias and uncertainty in the analytical model used to predict resistance.

The terms $m_{B}$ and $V_{B}$ may be interpreted as the bias and variability in the resistance that remain when the values of all basic variables in the predictive model are known. These may be estimated from laboratory tests 
of structural elements or systems in which the yield stress of steels or crushing strength of concrete are determined by testing companton specimens and for which the actual dimensions are known. Test data frequently are presented as. the ratio of test to calculated strength, $R / R_{m}$. Then,

$$
\begin{aligned}
& m_{B}=m_{R / R_{m}} \\
& v_{B}=v_{R / R_{m}}
\end{aligned}
$$

Actualiy, the $m_{B}$ and $V_{B}$ values computed from this procedure include a within-batch bias and variability, $m_{W B}$ and $V_{W B}$. For example, the yield stress of reinforcement varies somewhat along the length of a bar so that even if a companion specimen is cut from the bar before it is placed in the test structural member, the yield stress at the point(s) of structural failure may differ slightly from the companion specimen strength. Monte Carlo simulations [Ellingwood, et a7, 1980] have shown that this source of bias and variability is usually quite small ( $m_{W B} \approx 1.00$ and $v_{W B} \approx 0.04$ or less). Thus, for most structural modeling formulations of practical interest, the bias and variability to be used in reliability analysis can be taken as,

$$
\begin{aligned}
& \mathrm{m}_{B} \approx 1.0 \mathrm{~m}_{\mathrm{R} / \mathrm{R}_{\mathrm{m}}} \\
& \mathrm{v}_{\mathrm{B}} \approx\left[\mathrm{v}_{\mathrm{R} / \mathrm{R}_{\mathrm{m}}}^{2}-0.04^{2}\right]^{1 / 2}
\end{aligned}
$$

Note that while it is conservative to ignore the effect of within-batch variability, an appreciation of its effect is important. For those cases where an unusually accurate prediction model is used, as may be the case with nuclear structures, it may be overly conservative to ignore the withinbatch variability.

The probability distribution for B can be usually be assumed to be normal. 


\subsubsection{Hot-Rolled Structural Steel}

A significant data base exists to characterize yield stress, tensile strength, modulus of elasticity, and Poisson's ratio of plate material and tensile and shear strength of high strength bolts and welds [Galambos, 1978]. Most of these data have been collected and summarized as part of an extensive research program at Washington University to develop probability based criteria for steel design. A summary of these data is presented in Table 3.2. These data have been used, along with data on modeling of capacity, to determine resistance statistics for hot-rolled structural steel elements, some of which are summarized in Table 3.3.

\subsubsection{Reinforced Concrete}

The strength of reinforced concrete structural members depends on the strength of concrete in compression and tension, the yield stress in the reinforcement, the accuracy in placing reinforcement, and the cross sectional dimensions. Additional uncertainties arise from the formulas used to compute member strengths.

Statistical data on the crushing and tensile strengths of concrete, yield stress and ultimate strength of reinforcement, and structural member dimensions have been gathered in research programs at the National Bureau of Standards and the University of Alberta [Ellingwood, 1978; Mirza \& MacGregor, 1976]. These data are representative for ordinary structures built with "average" quality control conditions. A representative sample of such data is summarized in Table 3.4. The statistics of beam and column capacity, summarized in Table 3.5, have been obtained using Monte Carlo simulation. Very little work of a similar nature has been done for slabs, shells, joints or structural systems. 


\subsubsection{Limitations of Existing Resistance Data}

Most of the aforementioned data pertains to grades of concrete and structural steel and to connections that are common in ordinary structures. To the extent that such materials and structural elements are used in nuclear structures, their statistics should also be valid for the reliability analysis of nuclear structures. Statistics that are dependent on the quality control, such as concrete compressive strength and dimensions, would be different for nuclear structures. Additional data representative conditions prevalent at nuclear plant sites needs to be gathered.

The statistics presented in Tables 3.2 through 3.5 are valid for situations involving static or slowly increasing loadings. Effects on resistance due to either increased rate of laod application or short load durations may require further considerations before the data can be fully utilized. More, structural resistances to earthquake, sudden pressures, and missile impacts, may depend as much on the energy-absorbing capacity of the material, as on actual ultimate strength. Additional members will have to be developed to incorporate ductility effects into the calculation for component "resistance" statistics.

In carrying out reliability analyses of ordinary structures, the statistical properties in terms of strength of structural elements within a system have been emphasized rather than the statistical properties of the system as a whole. Because of this, a significant effort has been devoted to identifying appropriate resistance functions $R_{m}$ and $R$ and modeling variables $B$ to describe failure for individual structural elements. It is implied in this approach that the resistance in a member can be described fully by the resistance at some critical point (in statistical terms, the resistances at 
any two points within a member are perfectly correlated) and that failure at that critical point is tantamount to failure of the member. Since this type of member safety checking procedure has also been followed in conventional deterministic design (an exception is the kinematic mechanism analysis permitted by Part 2 of the AISC Specifications), it is not surprising that this approach has also been carried over into the firstgeneration probability-based design.

If probabilistic statements regarding the performance of systems of structural elements are to be used as a basis for decision-making, the required component reliabilities must be inferred from system reliability requirements before a probability Dased criteria for the design of the components can be recommended. This requires some knowledge of system behavior, as described in the following section.

\section{3 Models of System Behavior}

As described in greater detail in Section 4, reliability analysis and probability-based design criteria require reasonably accurate mathematical models for calculating element and system behavior for various postulated load conditions. The general approach is to develop models based on the principles of mechanics and to use whatever experimental data are available to validate and refine the analysis. These comparisons of predicted and observed behavior are also used to define the statistical nature of the analysis factor $C_{i}$ in $E q .3 .1$. The models are then used to calculate reliabilities and to generate probability based criteria for design. As mentioned in the previous section, these models are reasonably well developed 
for simple structural elements and systems, to the point where mean $\bar{C}_{\mathbf{j}}$ may simply be taken as the analysis factor and the coefficient of varfation $v_{C_{j}}=0.05$ or less.

Complex finite element computer codes have been developed and used for analyzing the behavior of nuclear structures. The theoretical formulation upon which these codes are based requires an approximation of the displacement field by so-called "shape functions" which are usually composed of truncated (quadratic or cubic) polynominals. The magnitude of error arising from this approximation depends upon the severity of the loading and the resulting stress-strain fields, the type of finite element used (plane stress, plane strain, axisymmetric, plate, shell, etc.), and the size of the elements. Furthermore, practical applications of these codes require additional approximations and idealizations of the structural geometry, material properties, external loadings, and boundary conditions. Complex structures such as category I seismic structures are composed of very elaborate geometrical configurations fabricated from a variety of materials, and are subjected to complicated sets of static and dynamic loads and a variety of boundary conditions. A typical case would be the Mark III containment shown in Figure 3.1. Obviously, it is not realistic or practical to model every detail of this structure exactly and hence simplifications must be made regarding both the geometry as we11 as the material properties. For instance, the actual geometricä 1 configuration may be replaced by an idealized three-dimensional or axisymmetric geometry, the reinforced concrete may be represented by an equivalent orthtropic material, and so forth. However, all such simplifications introduce errors in the analytical results which vary according to the level of simplifications employed. Additional errors are introduced when complicated loads and boundary conditions are represented by simplified "nodal" constraints. 
For nonlinear and dynamic structural problems other sources of error, e.g., error resulting from linearization of the nonlinear equations or from time integration of the equations of motion, are also present.

In summary the accuracy of these models depends on (1) the finite element grid size, (2) the idealization of the structural and boundary conditions, (3) assumptions made in linearization of nonlinear equations, (4) assumptions of damping and number of modes used for dynamic analysis, and (5) the time-steps and dynamic reduction schemes chosen for time dependent problems. While the development of finite-element methods has progressed to the point that most of the codes available for the industry should be sufficiently accurate, there is a need for code verification by analytical and physical test benchmarks. An effort to determine variations caused by some of the above mentioned items will be made. 


\subsection{Reliability Analysis}

In general, reliability analysis of the structural system can be performed if the limit state modes of the system can be identified, and if the joint density function of the random variables which specify the various limit state modes can be determined and integrated over the safe domain. A satisfactory design will be achieved by selecting a combination of the load and resistance variables to meet the prescribed target reliability. The theoretical aspects of the reliability analysis is discussed in detail in Section 4.

The reliability analys is of nuclear structures encounter a great range of complexity here-to-fore not considered for conventional structures. In the following, we will discuss the complexity briefly.

\subsubsection{System and Component Reliability}

As mentioned previously, reliability analyses of conventional structures have focused on element behavior rather than system behavior and thus significant amount of effort has been expended into calculating probabilities of failure of simple steel and concrete beams and columns. However, the nuclear structures are more complex, and we must consider the system reliability of the structures. The reliability of a system depends not only on the relabilities of its components but also on the nature of the correlation in behavior among the components. This latter item may be significant but is frequently is unkown. Procedures for constructing bounds on the reliability of a system in terms of component reliabilities are available [Corne11, 1968; Freudenthal, et a1., 1966]. However, for nuclear structures that are designed for low probabilities of failure, these bounds frequently are too wide to be useful for structural safety-related descision making. 
System reliability analysis for a complex structure always involves decomposing the system into various events which comprise the probability of success or failure of various physical entitilies: components, elements, etc. The end result is to formulate an equation for the probability of system success, $P_{S}$, which is the reliability $\left(R=P_{S}\right)$ or the limit state probability of system failure, $P_{f},\left(P_{f}=1-P_{s}=1-R\right)$.

The conventional methods of decomposing a system into elements involve one of two techniques. The first technique is to formulate a set of mutually exclusive events with the aid of failure modes analysis or event trees. The second technique decomposes the system into components, and relates the system reliabllity to the component reliabilities using reliability block diagrams or fault trees. The second appraoch is the common one used in electrical or mechanical systems, where the iteractions among the components are second order effects and the statistical independence can generally be assumed to hold.

In the case of structural systems, the interactions among elements are strong and a different approach is needed. We return to method one and decompose the system into a set of mutually exclusive limit state. We must separately calculate the probability of failure for each of these limit states using the techniques of Chapter 4 . The method for combining these individual limit state probabilities to achieve the system reliability goal is discussed in Appendix $C$. 


\subsubsection{Interpretation of Small Probabilities}

Because of the extraordinary potential consequences of a failure in a nuclear structure, the probability of structural failure should usually be less than in ordinary structures. According to current design philosophy, the failure of a structural component should give rise to a probability of radioactive release that is less than $10^{-7}$ /year. Such small probabilities are so far outside the realm of observation that it is difficult to use them as the sole basis for decision making, particularly when they are calculated from idealized postulated models [freudenthal, et al., 1966]. It may be useful to interpret small probabilities in a relative or Bayesian sense rather than in the classical relative frequency sense. In other words, if we can compute a limit state probability associated with some event for which we have knowledge, then we can relate probabilities for other events outside our experience to the "reference" probability and make engineering decisions on the basis of the relative rather than absolute probabilities. This approach has been used for the risk analyses of some ordinary structures. It has however, not been explored with respect to application for nuclear structures.

\subsubsection{Measures of Reliability}

Probability-based design has been used to develop the design criteria for ordinary buildings and structures [Galambos, 1978; Ellingwood, et al., 1980]. A common feature of these approaches is their dependence on first-order, second-moment reliability analysis and their reliance on the reliability index (safety index) as a measure of reliability (further discussion in Section 4) 
The safety index may be thought of as a measure of reliability in terms of the means and variances of the design variables in the limit state function. In generar, it may not be as informative a measure of reliability as the probability of failure because it does not incorporate the complete probability distributions in the safety analysis. Thus, it is possible that first-order second-moment reliability analysis may not be an acceptable procedure for the risk assessment of nuclear structures where low-probability events with extreme consequences are of interest.

It should be noted, however, that recent developments enable information on the probability distributions of the design variables to be incorporated into the calculation of the safety index. Moreover, the safety index so computed can be related to a limit state probability of failure through a simple relationship. Under these circumstances, the computation of the safety index may be viewed simply as an alternative to integrating numerically the joint probability density function to determine a probability of failure.

\subsubsection{Identification of Failure Events}

Reliability assessments of any structure require a definition of component or system failure. This definition is usually clear for ordinary buildings and structures, at least insofar as codes, standards, and regulatory authorities are concerned. It is usually related to occupant safety. Included in these safety-related limit states would be loss of load-carrying capacity of structural members or a large portion 
of the structure. Functional requirements for the building under conditions of normal use generally receive much less attention in building codes and standards. The current regulatory emphasis for nuclear structures tends to concentrate on safety-related functional capability [Howard, 1976] rather than on the collapse, gross deformation or other ultimate limit state tacitly considered in the design of ordinary buildings and structures [ANS A58, 1972; ACI 318, 1977; AISC, 1978].

A distinguishing feature of the functional requirements is the fact that, unlike ultimate limit states, there is no discrete condition of failure. For example, the point at which the width of cracks in reinforced concrete shear walls or the deflections in a steel frame become objectionable is not clear. Similarly, a valve may stick a part of the way open and may still continue to perform its intended function during certain events, while for other events its function would be unsatisfactory.

When failure events and probabilities of failure are discussed in subsequent sections, it should be clearly understood that the failures could be functional, as well as catastrophic.

\subsubsection{Linearity}

In the studies of the reliability of nuclear structures to be performed in this program, it will be assumed initially that the structures and components behave in a linear fashion up to the point of ailure and that the loads can be combined in a linear fashion. The present regulation for nuclear structures concentratesmore on safety-related functional capability than an ultimate limit state. Ultimate load carrying capacity however usually entails significant nonlinear behavior. This behavior may be relatively unimportant if safety-related functions are impaired before arriving at this state. More specifically, if radiation leakage occurs, then the structure has failed, even if it continues to have additional load-carrying capacity. 
Thus, questions of nonlinearities in combining structural responses to loads will be deferred until linear behavior has been thoroughly assessed. While at this time it is believed that this is an appropriate way to approach the reliability calculations, it may be desirable to evaluate nonlinear effects at some later stage in the research program. This question will be reexamined when current test programs on safety margins at ultimate load have been completed [Preliminary results were presented at 8th Light Water Reactor Safety Meeting, National Bureau of Standards, October, 1980].

\subsection{Specification of Safety Criteria}

The development of probability-based safety specifications for nuclear structures involves the selection of an acceptable failure probability or reliability, the selection of an appropriate safety checking format, and the calculation of a set of load factors and load combinations that make it possible to achieve the acceptable risk, (within certain tolerances), for all likely design situations. This represents a significant step beyond the current use of probabilistic methods in nuclear safety specifications. Their use in the nuclear field has been mostly limited to the decision process, providing judgment as to where postulated loads should be included or excluded form the load analysis [Stevenson, 1980]. The following sections discuss some particular problem areas related to this approach. 


\subsubsection{Target Reliabilities}

The selection of acceptable risk levels or desired (target) reliabilities should depend on the mode of failure (does the failure develop slowly? Is there prior warning to take remedial action, or does it occur suddenly?) and the consequence of failure (radioactive release, extended withdrawal from service, costly repairs). The target reliabilities may not necessarily be the same for all faiture criteria and load combinations if the consequence of one is demonstrably more severe than another.

In the development of probability-based design criteria for ordinary structures, heavy reliance has been placed on reliabilities associated with existing specifications [Galambos, 1978; Ellingwood, et al, 1980]. Extensive calculations have been performed to determine measures of reliability for simple steel and reinforced concrete beams and columns for which performance presumably has been satisfactory in the past. This approach has the advantages that it references the reliability based design procedure to conditions that been satisfactory in the past and it avoids the need to specify explicitly an acceptable risk. However, the process of code monitoring and adjustment that is used to gradually adjust the reliabilities inherent in existing conventional design specifications is not effective for nuclear or other essentially unique structures. There is insufficient experience with the performance of nuclear structures under extreme events to reliably calibrate target reliability levels with those of exisitng specifications. Thus, the selection of appropriate target reliability levels must be much more deliberate and certainly will be more difficult.

For the design of nuclear structures, it is anticipated that target reliability levels would be set by the regulatory authority. Thus, this research is intended to provide a matrix of load factors and combinations 
that depend on a reliability level. This will also be of value in demonstrating the relationship between reliability and structural design cost. A procedure will provided to enable the establishment of related system and component reliabilities.

\subsubsection{Code Formats}

A significant amount of research on alternate code formats is available. There invariably is an optimizing process, whether explicit or implicit, that takes prace between the conflicting needs of rigor and simplicity when selecting a suitable safety criteria format. It is assumed that the basic safety checking equation will take the form of Eq. 2.1, and that because of the relatively higher cost and potential hazard, some increase in the level of criteria complexity over that of for ordinary structures is acceptable.

A few possible safety checking equation formats are given in the following pages to emphasize the practical nature of the end product of this program. It is important to point out that while there may be no significant change from viewpoint of the designer, the safety factors can be made rational and reproducible by others, given the same sources of data. This is a significant improvement over current design criteria.

Numerous formats are possible for the design equation. In one of the simplest, a single load factor is applied to the sum of the load effects, while a single resistance factor is applied to material strength or to structural capacity. This yields,

$$
\phi R \geq \gamma\left(Q_{1}+Q_{2}+\ldots\right)
$$


In this and the following design equations, we actually mean a set of equations in which the $\phi$ and $\gamma$ would depend on how the loads are being combined and on the limit state. The loads could be combined according to the square root sum of the squares, (SRSS), or some other procedure. This format is currently used (with $\gamma=1.0$ for all load combinations) in working stress design. However, it does not guarantee a consistent reliability for all components because it applies the same load factor to loads in a particular combination that are highly variable and to those that are relatively predictable. Moreover, it may be unsafe especially when the load effects counteract one another, since one factor of safety may then be applied to a small difference between tow large numbers.

A second design equation format takes the form [ACI 318, 1977; NBC, 1977],

$$
\begin{aligned}
& \phi R \geq \psi\left(\gamma_{D} D+r_{1} Q_{1}+r_{2} Q_{2}+\ldots\right) \\
& \phi R \geq \gamma_{D} D+\psi\left(r_{1} Q_{1}+r_{2} Q_{2}+\ldots\right)
\end{aligned}
$$

in which $\gamma_{D} D=$ factored permanent (essentially time-invariant) 1oad, $\gamma_{i} Q_{i}=$ factored time-varying loads, and $\psi=$ load combination probability factor that accounts for the small probability that more than one time-varying load attains its lifetime maximum value simultaneously. Eq. 3.16a and $3.16 \mathrm{~b}$ offer an improvement over Eq. 3.15, in that separate load factors are applied to the individual loads. This makes it possible to achieve more uniform reliability over all ranges of $Q_{j} / Q_{j}$ if $Q_{i}$ and $Q_{j}$ have different variabilities. However, Eqs. 3.16 may not be a particularly acccurate representation of the way loads combine in a structure, particularly at states approaching the ultimate condition. In particular, it makes no sense to apply $\psi$ to the dead load as in Eq. 3.16 a, since dead load is always present. 
Experience with structural failures in building suggests that for failures that do not arise during construction or from negligence, the maximum total load effect is usually achieved when one of the time-varying loads attains its maximum value while the other loads are at their "pointin-time" values. This suggests that the design criteria should take the form,

$$
\phi R \geq \gamma_{D} D+\gamma_{\max } Q_{\max }+\Sigma \gamma_{i} Q_{i}
$$

in which $Q_{\max }=$ the principal time-varying load in the combination and $\gamma_{i} Q_{i}=$ factored point-in-time values to be combined with $\gamma_{\max } Q_{\max }$. In comparing Eq. 3.17 with Eqs. 3.16, $\gamma_{\max } Q_{\max }$ in Eq. 3.17 would be of the same order of magnitude as $\gamma_{i} Q_{i}$ in Eq. 3.16 [Ellingwood, et al, 1980]. The individual terms in the summation term of Eq. 3.17 would be less than the corresponding terms in Eqs. 3.16a and 3.16b, but there would be no load combination probability factor. Interestingly, the format of Eq. 3.17 has also been suggested in a number of theoretical load studies [Turkstra, 1970; Larabee, 1979] and appears to agree well with Monte Carlo simulations of a number of realistic load processes and load situations.

The load and resistance factors in Eq. 3.17 may be determined by applying the reliability analysis procedures described in Chapter 4 (Eqs. $4.29-4.31$, et seq). In general, $\phi$ and $\gamma_{j}$ would depend on the measure of component reliability $\left(p_{f}\right.$ or $\left.\beta\right)$ and on the probability distributions assumed for the variables in the limit state equation (or, at least, their means and variances). It should not be surprising that when the reliability measure is fixed, there is significant variation in the $\phi$ and $\gamma_{\mathbf{i}}$ that are calculated for different design situations for which Eq. 3.17 should apply (different span-depth ratios in flexural members, percentage reinforcement 
in concrete members, percentage of the total load effect arising from dead, live, transient loads, etc,), [Ellingwood, 1978; 1980], For practica] design applications, these variations ought to be eliminated on minimized.

A set of $\phi$ and $\gamma_{i}$ may be determined that are optimal, in some sense, when all probable design situations are considered. These load and resistance factors will depend on the format selected for the design equation. When a format has been selected (e.g., Eq. 3.17, the load combination array in ACI 349 , etc.) and $\phi$ and $r_{i}$ are assigned specific numerical values, then a value of $p_{f}$ can be computed for each design situation which, in general, will differ from the target probability $p_{f T}$. The task is then is then to determine the specific $\phi$ and $\gamma_{j}$ that minimize the difference between $p_{f}$ and $p_{f T}$ when all likely design situations are considered. In equation form, the problem is to minimize

$$
I\left(\phi, \gamma_{i}\right)=\sum_{k} w_{k} h\left(p_{f_{k}}, p_{f_{T}}\right)
$$

in which $p_{f_{k}}$ is the probability of failure for design situation $k$ and $w_{k}$ is the relative likelihood of situation $k$. Function $h(a, b)$ is some measure of the closeness between $a$ and $b$ and may be selected to penaiize overdesign and underdesign equaliy [e.g., $\left.h()=(b-a)^{2}\right]$ or to penalize underdesign more than overdesign. The selection of a suitable function will be considered subsequently.

Although it is too early in this program to make specific recommendations for a safety checking equation format, it seems reasonable to assume that the probability-based design specification will have, (at least initially), an appearance that is similar to one of the above equations. It should be pointed out however, when the response is nonlinear, it may be uncon ervative to factor the load effect; the load should be factored prior to performing the safety check. Moreover, when several of the loads in the combination are dynamic, as is frequently the case with nuclear 
structures, a load criterion in a form other than the absolute sum of design loads may be appropriate. It is widely recognized [ANS A58, 1972] that when several time-varying loads act on a structure, the probability that two or more will attain their design values simultaneousiy is very small and some reduction in their effect may be appropriate. The question as to what the reduced combination should be for two or more dynamic loads has not yet been fully considered. One suggestion has been to take the square root of the sum of squares (SRSS) of the design load effects. Other possibilities will also be considered and reported upon in a subsequent scheduled report.

\subsubsection{Design Loads}

The current grouping of structural loads in the Standard Review Plan into normal operating loads, severe and extreme environmental loads, and abnormal loads, and the specification of load combination involving these loads, is far more complex than that found in load standards governing design of ordinary buildings. One of the results of such a classification is that different load levels for the same basic environmental effect may be specified and that these different levels are tied to specific states of the structure.

An example of this is the specification for the operating basis earthquake (OBE) force $E_{0}$ and the safe shutdown earthquake (SSE) force $E_{S}$. The structure is expected to maintain its integrity under $E_{S}$ and to continue normal operation under $E_{0}$. These correspond to different fractiles of the same basic load effect. No standard or building code for ordinary structures in the United States contains such a two-level load specification. The question as to whether such a specification is desirable and under which circumstances it should or could be modified, has not yet been addressed. 


\subsubsection{Design Philosophy}

The differences in design philosophy upon which the existing sections of Standard Review Plan are based introduce an additional level of complexity into the design of nuclear structures. Consider, for example, a Category I structure having both steel and reinforced concrete components. Standard Review Plan 3.8.4 specifies one matrix of load combinations for steel design in which the loads are all unfactored and a second matrix of load combinations for reinforced concrete design in which the loads are factored. Thus, two separate load and structural analyses must be performed for the structural system that calls for a mix of steel and reinforced concrete structural elements. Not only does this unnecessarily complicate the design, it is a potential source for error. Moreover, there is no assurance that these two sets of load combinations are sufficient to assure uniform reliability and performance for steel and concrete elements within the system.

This potential for confusion could be reduced considerably by specifying one set of loads, load factors, and load combinations that would be used for the design of all structural elements within nuclear structures regardless, whether they were composed of stee1, reinforced or prestressed concrete or any combination thereof. In other words, the right hand side of Eq. 2.1 would be the same in Standard Review Plans 3.8.1 through 3.8.4. Only one load analysis would be needed for each structural system. Each. Standard Review plan section would need to specify appropriate resistance 
criteria (the left hand side of section Eq. 2.1) to be used in checking against the effect of the factored loads. These would depend on the mode, the consequence to the remaining structure due to the failure of the structural element, the quality control in construction, whether or not the element is inspected during its service life, and perhaps other factors.

Such a unified approach to structural design is rapidly gaining acceptance in standards committees charged with drafting provisions for ordinary buildings. There should be significant advantages to such an approach for nuclear structures as we11. Our primary goal, of course, is to develop probability-based load factors and load combinations that could be used in conjunction with sections 3.8.1 - 3.8.4 of the Standard Review Plan. Initially this may require separate load criteria for each SRP section. However, the feasibility of developing a single load criteria, that could be used with all the above mentioned SRP sections and would thus harmonize the design of nuclear structures, will also be examined. 
Table 3.1. Availability of Structural Load Data for Probability Analysis

Load Type

Dead

SRV discharge

Operating live

Snow

Ice

Rain

Wind

Extratropical

Hurricane

Tornado

Pressures

Pressure drop

Missiles

Earthquake

Abnormal

\section{Status Code}

1

2

3

2

2

2

1

2

2

3

3

2

3

Status Code

1. Ready to use in probability based design.

2. Raw data available, some further analysis required.

3. Raw data unavailable or of questionable quality. 
Table 3.2. Typical Basic Statistics for Steel Properties

\begin{tabular}{|l|l|c|}
\hline \multicolumn{1}{|c|}{ Property } & Mean & Coefficient of Variation \\
\hline Static yield stress, webs & $1.10 \mathrm{~F}_{y}$ & 0.11 \\
$\begin{array}{l}\text { Modulus of elasticity } \\
\text { or shear modulus }\end{array}$ & $1.0 \mathrm{E}$ or $\mathrm{G}$ & 0.06 \\
Tensile strength of steel & $1.10 \mathrm{~F}_{u}$ & 0.11 \\
Poisson's ratio & 0.3 & 0.03 \\
\hline
\end{tabular}

${ }^{\star} F_{y}, F_{u}, E, G=$ nominal value specified by AISC.

Table 3.3. Typical Resistance Statistics for Steel Structural Members

\begin{tabular}{|l|c|c|}
\hline \multicolumn{1}{|c|}{ Member } & Mean $^{{ }^{*}}$ & Coefficient of Variation \\
\hline Tension member, yield & $1.05 \mathrm{~F}_{y^{\mathrm{A}}}$ & 0.11 \\
Tension member, ultimate & $1.10 \mathrm{~F}_{\mathrm{u}^{\mathrm{A}}}$ & 0.11 \\
$\begin{array}{l}\text { Compact beam, uniform } \\
\text { moment }\end{array}$ & $1.07 \mathrm{~F}_{y^{\mathrm{Z}}}$ & 0.13 \\
Column, intermediate & $1.08 \mathrm{\sigma}_{\mathrm{cr}}^{\mathrm{A}}$ & 0.15 \\
\hline
\end{tabular}

${ }^{*} F_{y}, F_{u}, \sigma_{c r} A, Z=$ nominal values specified by AISC. 
Table 3.4. Typical Basic Statistics for Reinforced Concrete Elements

\begin{tabular}{|c|c|c|}
\hline \multicolumn{1}{|c|}{ Property } & Mean * & $\begin{array}{c}\text { Coefficient } \\
\text { of Variation }\end{array}$ \\
\hline $\begin{array}{c}\text { Concrete compressive strength } \\
\text { in situ }\end{array}$ & $\begin{array}{c}\text { varies } \\
0.8 \mathrm{f}_{\mathrm{c}}{ }_{\mathrm{c}} \text { typical }\end{array}$ & $0.18-0.20$ \\
$\begin{array}{c}\text { Reinforcement } \\
\text { Grade 60 bars, static yield } \\
\text { stress } \\
\text { Grade 270 strand, tensile } \\
\text { strength }\end{array}$ & $67 \mathrm{ksi}$ & 0.10 \\
$\begin{array}{c}\text { Dimensions } \\
\text { Overal1 dimensions of members } \\
\text { Placement of reinforcement }\end{array}$ & $281 \mathrm{ksi}$ & 0.03 \\
\hline
\end{tabular}

${ }^{*} d_{n}, h_{n}=$ nominal dimension on working drawings.

Table 3.5 Typical Resistance Statistics for Reinforced Concrete Structural Members

\begin{tabular}{|l|c|c|}
\hline \multicolumn{1}{|c|}{ Member } & Mean $^{*}$ & Coefficient of Variation \\
\hline RC Beam, Flexure & $1.12 \mathrm{M}_{u}$ & 0.13 \\
Two-way slabs, flexure & $1.16 \mathrm{M}_{u}$ & 0.15 \\
$\begin{array}{l}\text { Prestressed concrete beam, } \\
\text { flexure }\end{array}$ & $1.05 \mathrm{M}_{\mathrm{u}}$ & 0.05 \\
RC Beam, shear & $1.10 \mathrm{v}_{\mathrm{u}}$ & 0.20 \\
$\begin{array}{l}\text { Short columns, compression } \\
\text { failure }\end{array}$ & $0.95 \mathrm{R}_{\mathrm{u}}$ & 0.15 \\
\hline
\end{tabular}

$\star_{u}, V_{u}, R_{u}$ computed according to ACI Standard 318 . 


\section{ANALYSIS OF RELIABILITY OF STRUCTURES*}

\subsection{Introduction}

The conceptual framework for probability based design is provided by the classical theory of reliability [Freudenthal et al., 1966; Ang and Cornell, 1974]. In this section, some of the more significant recent developments in the area of structural reliability analysis are reviewed and new interpretations for some of these crucial theoretical developments are proposed.

A matheriatical model is first derived from principles of mechanics and experimental data which relates the resistance and load variables for the operating, accidental and environmental loading conditions of interest. Suppose that this relation, known as a limit state surface, is siven by

$$
g(\underline{x})=g\left(x_{1}, x_{2}, \ldots, x_{n}\right)=0
$$

where $x_{i}=$ the (generally random) resistance or load variables. By definition, the limit state is reached when

$$
g(\underline{x})=g\left(x_{1}, x_{2}, \ldots, x_{n}\right) \leq 0
$$

In a generic sense, failure means that the structure, or a component thereof, ceases to fulfill its intended purpose. Usually, a number of limit states can be identified for a structure, with each representing a state of either ultimate structural failure, structural unserviceability or operational malfunction. For an overall safety analysis, these limit states must be properly combined and the resulting combined limit states and associated limit state surfaces (e.g., for the global state of ultimate structural failure) should be obtained, in principle, in the same general analytical expressions as given A substantial portion of this section is adapted from Shinozuka [1980c]. 
in Eqs. 4.2 and 4.1 , respectively.

The probability $P_{f}$ that a limit state, individual or combined, will be reached is then referred to as the limit state probability ${ }^{\star}$ and given by

$$
P_{f}=\int_{D} f_{\underline{X}}(\underline{\xi}) d \underline{\xi}
$$

where $f_{\underline{X}}(\underline{\xi})=f_{X_{1}} x_{2} \ldots x_{n}\left(\xi_{1}, \xi_{2}, \ldots, \xi_{n}\right)$ is the joint density function of $X_{1}, x_{2}, \ldots, x_{n}, D$ is the domain in the $n$-dimensional space in which Eq.

4.2 is satisfied, and $d \underline{\xi}=d \xi_{1} d \xi_{2} \ldots d \xi_{n}$.

In the simplest application where the failure criterion is considered to contain just two variables: a resistance $R$ and a load effect $Q$, dimensionally consistent with and statistically independent of $R$, the failure event is specified by $R-Q \leqq 0$ and the probability of failure ${ }^{\star} P_{f}$ is computed as

$$
P_{f}=P(R \leqq Q)=\int_{-\infty}^{\infty} F_{R}(x) f_{Q}(x) d x
$$

in which $F_{R}(\cdot)$ = the cumulative probability distribution function (cdf) in $R$ and $f_{Q}(\cdot)=$ the probability density function (pdf) for $Q$. If both $R$ and $Q$ have normal distributions, for example, then

$$
P_{f}=\Phi\left(-\frac{\bar{R}-\bar{Q}}{\sqrt{\sigma_{R}^{2}+\sigma_{Q}^{2}}}\right)
$$

where $\bar{R}(\bar{Q})$ and $\sigma_{R}\left(\sigma_{Q}\right)=$ the mean and standard deviation of $R(Q)$, respectively, $\Phi(\cdot)=$ the standardized normal distribution function. The probability of fallure $P_{f}$ in Eq. 4.5 is represented by the shaded area in Fig. $4.1 \mathrm{a}$. If both $R$ and $Q$ have lognormal distributions with coefficients of variation, $V_{R}$ and $V_{Q}$, respectively, then

* "Limit state probability" and "Probability of failure" are interchangeably used throughout. 


$$
P_{f}=\Phi\left(-\frac{\ln (\bar{R} / \bar{Q})}{\sqrt{V_{R}^{2}+V_{Q}^{2}}}\right)
$$

if $v_{R}^{2}$ and $v_{Q}^{2}$ are both less than or equal to approximately 0.1 . The probability of failure $P_{f}$ in Eq. 4.6 corresponds to the shaded area in Fig. 4.1b.

In probability-based design, $P_{f}$ serves as a quantitative measure of structural reliability. However, it is well known that there are two major difficulties associated with the use of Eq. 4.3. (1) It is an impractical proposition to construct the joint density function on the existing data base which is usually scarce and insufficient particularly for the crucial range of large variable deviations, and (2) practically for all cases of engineering interest, the multidimensional integration over the generally irregular domain $D$ is impossibie to carry out analytically and quite costly, if not totally impractical, to perform numerically.

\subsection{First-Order, Second-Moment Methods}

In an attempt to circumvent these difficulties, First-Order, Second-Moment (FOSM) reliability analys is methods [e.g., Rackwitz, 1976] have been developed, in which the random variables are characterized by their first and second moments. While any continuous mathematical form of the limit state equation is possible, it must be linearized at some point for the purpose of performing the FOSM reliability analysis. The linearized version of the failure criterion defined by Eq. 4.2 is

$$
z=g\left(x_{1}^{*}, x_{2}^{*}, \ldots, x_{n}^{*}\right)+\left[\left(x_{i}-x_{i}^{*}\right)\left(\frac{\partial g}{\partial x_{i}}\right)_{\underline{x^{*}}} \leq 0\right.
$$

where $\left(\frac{\hat{\partial g}}{\partial x_{i}}\right)_{\underline{x}}$ indicates the derivatives evaluated at the linearizing point. 
Equation 4.7 is equivolent to the Taylor expansion of Eq. 4.2 about the point $\underline{x}_{\star}=\left(x_{1}^{\star}, x_{2}^{\star}, \ldots, x_{n}^{\star}\right)$ up to the linear terms. In this sense, the point $\underline{x}_{\star}$ is referred to as the linearizing point. The reliability analys is is then performed with respect to this 1 inearized version of Eq. 4.2.

In earlier structural reliability studies, the point $\underline{\bar{x}}=\left(\bar{x}_{1}, \bar{x}_{2}, \ldots\right.$. $\bar{x}_{n}$ ) representing the mean values of $x_{1}, x_{2}, \ldots, x_{n}$ was used as the 1 inearizing point. Assuming that the $X$-variables are statistically uncorrlated, the mean and standard deviation of $Z$ are approximated by

$$
\begin{aligned}
& \bar{z}=g\left(\bar{x}_{1}, \bar{x}_{2}, \ldots, \bar{x}_{n}\right) \\
& \sigma_{z}=\sum_{i}\left[\left(\frac{\partial g}{\partial x_{i}}\right)^{2} \quad \sigma_{x_{i}}^{2}\right]^{\frac{1}{2}}
\end{aligned}
$$

The extent to which Eqs. 4.8 and 4.9 are accurate depends on the effect of neglecting the higher order terms in Eq. 4.7 and the magnitudes of the coefficients of variation in $x_{i}$. If $g(\cdot)$ is linear and the variables are uncorrelated, Eqs. 4.8 and 4.9 are exact.

The reliability index ${ }^{\star} B$ is defined by

$$
B=\bar{Z} / \sigma_{Z}
$$

which is the reciprocal of the coefficient of variation (c.o.v.) in Z. Figure 4.1 shows the density functions of $Z$ for two alternate representations of the simple two-variable problem $Z=g(R, Q)$ discussed in the previous section (Eqs. $4.4-4.6$ ). The index $B$ is the distance from the mean value $\bar{Z}$ to the origin measured in the unit of standard deviation. As such, $B$ is a measure of the probability that $g(\underline{x})$ will be less than zero. Observe that in Fig. 4.1 that (1) if $\sigma_{R-Q}\left(\right.$ or $\sigma_{\ell n} R / Q$ ) remains constant, a positive shift in $\bar{Z}=$

* "Safety index" is also used interchangeably for "reliability index" throughout. 
$\bar{R}-\bar{Q}$ (or $\overline{l n} R / Q$ ) will move the density function to the right and (2) if the mean value $\bar{Z}$ is kept constant, the smaller standard deviation $\sigma_{Z}$ implies a greater concentration of the probability density around the mean, and note that both of these cases result in a reduced failure probability. Hence, an increase in $B$ leads to an increase in reliability. This conclusion generally applies to those cases where $R$ and $Q$ are governed by other than normal or lognormal distribution functions. Thus, the index $B$ has meaning as a measure of reliability and can be used to compare the relative safety of alternate designs even when the distribution functions of $R$ and $Q$ are not precisely known. However, it should be noted that safety statements are confined to those that can be made on the means and variances.

The index $B$ in Fig. $4.1 \mathrm{a}$ is given by

$$
\beta=\frac{\bar{R}-\bar{Q}}{\sqrt{\sigma_{R}^{2}+c_{Q}^{2}}}
$$

since $\overline{R-Q}=\bar{R}-\bar{Q}$ and $\sigma_{R-Q}=\overline{\sigma_{R}^{2}+\sigma_{Q}^{2}}$ provided that $R$ and $Q$ are uncorrelated. Alternatively, using the formulation of Fig. 4.16 and using the small variance approximations $\overline{\ln R / Q} \simeq \ln \bar{R} / \bar{Q}$ and $\sigma_{\text {ln } R / Q} \simeq\left(V_{R}^{2}+V_{Q}^{2}\right)^{\frac{1}{2}}$, one obtains

$$
\beta=\frac{\ln \bar{R} / \bar{Q}}{\bar{N}_{R}^{2}+V_{Q}^{2}}
$$

In fact, Eq. 4.11 was the basis for an early recommendation for a probability based structural code [Cornell, 1969] while Eq. 4.12 was the basis for the development of probability-based load and resistance factor design criteria for steel structures [Galambos, 1978].

It is noted that the probability of failure $P_{f}$ can be expressed as $\Phi(-B)$ 
using the $\beta$ given in Eq. 4.11 (Eq. 4.12) if both $R$ and $Q$ are normally (lognormally) distributed. Indeed, this is the result shown in Eq. 4.5 (Eq. 4.6). For this reason, one might refer to the $B$ in Eq. 4.11 (Eq. 4.12) as a normal distribution-based (log-normal distribution-based) reliability index.

Mean value FOSM methods have the following two basic shortcomings. First, the function $g(\underline{x})$ is linearized at the mean value point $\underline{x}$ of the $x$ variables. Therefore, when $g(\underline{X})$ is nonl inear, significant errors may be introduced (in particular, when approximating Eq. 4.1 by Eq. 4.7 ) at increased distances from the linearizing point $\underline{x}_{*}$ by neglecting the higher order terms. Moreover, it is important to observe that in most reliability problems of interest, the mean point $\underline{\bar{x}}$ is considerably removed from the limit state surface $g(\underline{x})=0$. Then, a significant difference may arise between the failure domains defined by $g(\underline{x}) \leqq 0$ in Eq. 4.3 and by its linearized version (with $\underline{\bar{X}}$ used as the linearizing point). The difference may be significant in the sense that the underlying probabilities of failure obtained from Eq. 4.2 using these two domains of integration are considerably different. The implication of this observation is that a linearization based on mean values is not the most desirable and that the $B$ associated with it may not represent the best safety index value. This difficulty is minimized if the function $g(\underline{X})$ is linearized at some point on the limit state surface $g(\underline{X})=0$. Second, mean vlaue methods fail to provide a reliability index $B$ that is invariant to mechanically equivalent but analytically different expressions describing a failure criterion. In effect, this means that $\beta$ depends on how the failure criterion is expressed analytically. This problem may be avoided if the function $g(\underline{x})$ is linearized at a point on the 1 imit state sufface. This is because $g(\underline{x})$ ant its partial derivatives in Eq. 4.7 are in- 
variant only on the surface $g(\underline{x})=0$ with respect to how the failure criteria are analytically expressed. The following method [Rackwitz 1976] of arriving at the $B$ value based on a linearized limit state equation expanded at a point on the limit state surface is known as an advanced FOSM method.

The procedure for selecting a suitable linearizing point can now be explained as follows. For ease of analytical development, all the variables are first transformed into their standardized forms:

$$
x_{i}=\left(x_{i}-\bar{x}_{i}\right) / \sigma_{i}
$$

where $\bar{x}_{j}$ and $\sigma_{i}$ are, respectively, the expected value and standard deviation of $x_{i}$. Obviously, the expected value and standard deviation of $x_{j}$ are respectively, zero and unity. The limit state equation (Eq. 4.2) must then correspondingly be rewritten in terms of the standardized variables as follows:

$$
g_{1}(\underline{x})=g_{1}\left(x_{1}, x_{2}, \ldots, x_{n}\right) \leqq 0
$$

This is illustrated in Figs. $4.2 \mathrm{a}$ and $4.2 \mathrm{~b}$ considering the case when $n=2$. Consider a point

$$
\underline{x}_{0}=\left[x_{1}^{0} x_{2}^{0} \ldots x_{n}^{0}\right]^{\top}
$$

on the limit state surface

$$
g_{1}\left(\underline{x}_{0}\right)=g_{1}\left(x_{1}^{0}, x_{2}^{0}, \ldots, x_{n}^{0}\right)=0
$$

and expand $g_{1}(\underline{x})$ into a Taylor series around $\underline{x}_{0}$ :

$$
g_{1}(\underline{x})=\sum_{k=0}^{\infty} \frac{1}{k !}\left\{\sum_{i=1}^{n} \Delta x_{i}^{0} \frac{\partial}{\partial x_{i}}\right\}_{0}^{k} g_{1}(\underline{x})
$$

where $\Delta x_{i}^{0}=x_{i}-x_{i}^{0}$, and the subscript 0 after the closing bracket indicates that all the derivatives are to be evaluated at $\underline{x}_{0}$. (This notation indicating the point at which derivatives are to be evaluated, is used henceforth). 
The following linear approximation $g_{0}(\underline{x})$ of the limit state function $g_{1}(\underline{x})$ is obtained from Eq. 4.17 by considering Eq. 4.16 and deleting those terms with $k \geq 2$ in Eq. 4.17:

$$
g_{0}(\underline{x})=\sum_{i=1}^{n} \Delta x_{i}^{0}\left(\frac{\partial g_{1}}{\partial x_{i}}\right)_{0}=\underline{\Delta x}_{0}^{\top} \underline{G}_{0}
$$

where

$$
\underline{\Delta x_{0}}=\left[\Delta x_{1}^{0} \Delta x_{2}^{0} \ldots \Delta x_{n}^{0}\right]^{\top}
$$

and

$$
\underline{G}_{0}=\left[\begin{array}{llll}
\left(\frac{\partial g_{1}}{\partial x_{1}}\right)_{0} & \left(\frac{\partial g_{1}}{\partial x_{2}^{-}}\right)_{0} & \ldots & \left(\frac{\partial g_{1}}{\partial x_{n}}\right)_{0}
\end{array}\right]^{\top}
$$

The expected value $\bar{g}_{0}(\underline{x})=\bar{g}_{0}$ and the standard deviation $\sigma_{g_{0}}$ of $g_{0}(\underline{x})$ are given by:

$$
\begin{aligned}
& \bar{g}_{0}=-\sum_{i=1}^{n} x_{i}^{0}\left(\frac{\partial g_{1}}{\partial x_{i}}\right)_{0}=-\underline{x}_{0}^{\top} \underline{G}_{0} \\
& \sigma_{g_{0}}=\left[\sum_{i=1}^{n}\left(\frac{\partial g_{1}}{\partial x_{i}}\right)_{0}^{2}\right]^{\frac{1}{2}}=\left(\underline{G}_{0}^{\top} \underline{G}_{0}\right)^{\frac{3}{2}}
\end{aligned}
$$

where statistical independence among the $x$ 's has been assumed. If $\beta_{0}$ is now constructed as before (cf., Eq. 4.10),

$$
B_{0}=\frac{\bar{g}_{0}}{\sigma_{g_{0}}}=-\frac{\underline{x}_{0}^{\top} \underline{G}_{0}}{\left(\underline{G}_{0}^{\top} \underline{G}_{0}\right)^{\frac{1}{2}}}
$$

then its value obviously depends on the point $\underline{x}_{0}$, around which the 1 imit state function $g_{1}(\underline{x})$ is expanded. Assuming that $g_{1}(\underline{x})$ is concave towards the 
origin and $E>0$, the minimum value $E$ of $\varepsilon_{0}$ is referred to as the reliability index. (If the limit state is safety-related, this is often termed the safety index). A corresponding point $\underline{x}_{0}$ is denoted by $\underline{x}_{\star}$ and designated either the design point or the checking point:

$$
B=\frac{\bar{g}_{\star}}{\sigma_{g_{\star}}}=-\frac{\underline{x}_{*}^{\top} \underline{G}_{\star}}{\left(\underline{G}_{\star}^{\top} \underline{G}_{\star}\right)^{\frac{3}{2}}}
$$

where

$$
\underline{G}_{*}=\left[\left(\frac{\partial g_{1}}{\partial x_{1}}\right)_{\star}\left(\frac{\partial g_{1}}{\partial x_{2}}\right)_{\star} \cdot \cdot\left(\frac{\partial g_{1}}{\partial x_{n}}\right)_{\star}\right]^{\top}
$$

The graphical interpretation of $B_{0}$ and $B(>0)$ is given in Fig. 4.3. The values of $\beta_{0}$ in Eq. 4.23 may be negative. If this happens, the (algebraically) smallest value $B$ of $B_{0}$ can be defined as the reliability index, under the assumption that $g_{1}(\underline{x})$ is convex towards the origin. However, the limit state probability implicitly associated with such a negative index is considered unacceptably high. A similar graphical interpretation of $\left|B_{0}\right|$ and $|B|$ given for positive $B_{0}$ and $B$ is possible. The preceding analysis suggests that the design point $\underline{x}_{\star}=\left(x_{1}^{\star}, x_{2}^{\star}, \ldots, x_{n}^{\star}\right)$ can be determined by solving the system of equations iteratively [Rackwitz, 1976]

$$
\begin{aligned}
& \alpha_{i}=\frac{\left(\partial g_{1} / \partial x_{i}\right)_{\star}}{\left[\left[\left(\partial g_{1} / \partial x_{i}\right)_{\star}^{2}\right]^{\frac{1}{2}}\right.} \\
& x_{i}^{\star}=-\alpha_{i}^{B} \\
& g_{1}\left(x_{1}^{*}, x_{2}^{\star}, \ldots, x_{n}^{\star}\right)=0
\end{aligned}
$$

searching for the direction cosines $\alpha_{j}$ which minimize $B$. In the original $X-$ 
variable space, the design point variables are given by

$$
\begin{aligned}
& x_{i}^{*}=\bar{x}_{i}\left(1-\alpha_{i} \beta V_{i}\right) \\
& g\left(x_{1}^{*}, x_{2}^{*}, \ldots, x_{n}^{*}\right)=0
\end{aligned}
$$

The set of points $\left(x_{1}^{*}, x_{2}^{*}, \ldots, x_{n}^{*}\right)$ will fall in the upper tails of the probability distributions for load parameters and the lower tails for resistance variables.

In the analysis of a given design, the procedure described above determines the $n+1$ values, $B, x_{1}^{*}, \ldots, x_{n}^{*}$, so that $g\left(x_{1}^{*}, \ldots, x_{n}^{*}\right)=0$ and $B$ is minimum. Conversely, if a target reliability index $B$ is specified in advance, a solution may be obtained for the design point $\underline{x}_{\star}$ so that $g_{1}\left(x_{1}^{*}, x_{2}^{*}\right.$, $\left.\ldots, x_{n}^{\star}\right)=0$, or $g\left(x_{1}^{\star}, x_{2}^{\star}, \ldots, x_{n}^{\star}\right)=0$, in terms of the original variables. For the purpose of practical design, however, the nominal design variables $x_{N_{i}}$ for $x_{i}$, specified by appropriate specifications and standards (e.g., $60 \mathrm{ksi}$ for the nominal yield strength of Grade 60 steel) are used together with the load and resistance factors $\gamma_{i}$ (depending on whether $x_{i}$ are load- or resistance-related variables) so that the limit state surface condition

$$
g\left(r_{1} x_{N_{1}}, r_{2} x_{N_{2}}, \ldots, r_{n} x_{N_{n}}\right)=0
$$

is satisfied. Through the interpretation that $x_{i}^{*}=\gamma_{i} x_{N_{i}}$, the factors $\gamma_{i}$ can be determined as $\gamma_{i}=x_{i}^{*} / x_{N_{i}}$

\subsection{Method of Lagrange Multipliers}

This section demonstrates that the safety index $B$ introduced in Eq. 4.24 can readily be obtained by making use of the method of Lagrange multipliers and that the corresponding design point is the location of maximum likeli- 
hood if Gaussian variabilities are assumed.

Consider the following optimization problem:

Minimize :

$$
\gamma_{0}=\left(\underline{x}^{\top} \underline{x}\right)^{\frac{1}{2}}
$$

subject to the constraint

$$
g_{1}(\underline{x})=0
$$

To solve this problem, a Lagrange multiplier $\lambda$ is introduced to construct $L$ :

$$
L=\left(\underline{x}^{\top} \underline{x}\right)^{\frac{1}{2}}+\lambda g_{1}(\underline{x})
$$

Setting $\partial L / \partial \underline{x}=\partial L / \partial \lambda=0$, the solution $\underline{x}_{*}$ and $\lambda^{\star}$ is obtained from

$$
\underline{x}_{*}=-\lambda^{*} \underline{r} \underline{G}_{\star}
$$

where $\gamma$ is the minimum of $\gamma_{0}$. Substituting Eq. 4.35 into Eq. 4.32 (with $\gamma_{0}$ replaced by $\gamma$ ),

$$
\lambda^{\star}= \pm\left(\underline{G}_{*}^{\top} \underline{G}_{*}\right)^{-\frac{1}{2}}
$$

where the choice for the sign is to be made later so that $\gamma$ is non-negative. Premultiplying both sides of Eq. 4.35 by $\underline{x}_{\star}^{\top}$ and using Eq. 4.36 , one obtains

$$
\gamma= \pm \frac{\underline{x}_{*}^{\top} \underline{G}_{*}}{\left(\underline{G}_{*}^{\top} \underline{G}_{*}\right)^{\frac{1 / 2}{2}}} \geqslant 0
$$

If $g_{1}(\underline{x})$ is expanded around $\underline{x}=\underline{x}_{\star}$ and the higher order terms are disregarded, a linear approximation similar to Eq. 4.18 is obtained as 


$$
g_{\star}(\underline{x})=\underline{\Delta x}_{\star}^{\top} \underline{G}_{\star}
$$

From Eq. 4.38 , it follows that

$$
\begin{aligned}
& \bar{g}_{\star}=-\underline{x}_{*}^{\top} \underline{G}_{\star} \\
& \sigma_{g_{\star}}=\left(\underline{G}_{*}^{\top} \underline{G}_{*}\right)^{\frac{1}{2}}
\end{aligned}
$$

If the safety index $\beta$ is defined on the basis of $\bar{g}_{\star}$ and $\sigma_{g_{\star}}$ as

$$
B=\frac{\bar{g}_{\star}}{\sigma_{g_{*}}}=-\frac{\underline{x}_{*}^{\top} \underline{G}_{*}}{\left(\underline{G}_{*}^{\top} \underline{G}_{*}\right)^{\frac{1}{2}}}
$$

then, the comparison between Eqs. 4.37 and 4.41 indicates that the safety in$\operatorname{dex}$ (or its absolute value) is the shortest distance between the origin ( $\underline{x}=$ $\underline{0}$ ) and the rimit state surface when $\underline{\underline{x}}_{*}^{\top} \underline{G}_{\star}<0$ (or when $\underline{x}_{*}^{\top} \underline{G}_{\star}>0$ ).

If the components of $\underline{x}$ are correlated and have a covariance (correlation) matrix $\Lambda$ (symmetric), a standardized random vector $\underline{y}$ whose components are uncorrelated can be constructed by the transformation:

$$
\underline{y}=\underline{A} \underline{x}
$$

provided that the transformation matrix satisfies

$$
\underline{A} \Lambda \underline{A}^{\top}=1 \quad \text { or } \quad \underline{\Lambda}=\underline{A}^{-1}\left(\underline{A}^{-1}\right)^{\top}
$$

where $I$ is an identity matrix of appropriate dimensions. In this case, along with the transformation introduced by Eq. 4.42, the limit state equation must also be transformed for the estimation of the limit state probabilities into

$$
g_{1}\left(\underline{A}^{-1} \underline{y}\right)=g_{2}(\underline{y}) \leqq 0
$$


Then, the random vector $y$, with uncorrelated components and the limit state equation in terms of $y$ (Eq. 4.44), form exactly the same analytical problem as solved for $\underline{x}$ and $g_{1}(\underline{x}) \leq 0$.

It is pointed out, however, that the safety index for the cases involving $\underline{x}$ with correlated components can also be derived without transforming $\underline{x}$ into y. Indeed, consider the following optimization problem (see Fig. 4.4):

Minimize

$$
r_{0}=\left(\underline{x}^{\top} \underline{\Lambda}^{-1} \underline{x}\right)^{\frac{3}{2}}
$$

subject to the constraint

$$
g_{1}(\underline{x})=0
$$

Again, construct a function $L$, introducing a Lagrange multiplier $\lambda$ :

$$
L=\left(\underline{x}^{\top} \underline{\Lambda}^{-1} \underline{x}\right)^{\frac{1}{2}}+\lambda g_{1}(\underline{x})
$$

Setting $\partial L / \partial x=\partial L / \partial \lambda=0$ for optimization, solution $\underline{x}_{*}$ and $\lambda^{\star}$ is obtained from

$$
\underline{x}_{*}=-\lambda^{\star} \underline{\underline{\Lambda}} \underline{G}_{*}
$$

Al gebraic manipulations similar to those that previously derived $\lambda^{*}$ and $\gamma$ in terms of $\underline{G}_{\star}$ and $\underline{x}_{\star}$ result, in this case, in

$$
\lambda^{*}= \pm\left(\underline{G}_{\star}^{\top} \underline{\Lambda} \underline{G}_{*}\right)^{-\frac{1}{2}}
$$

and

$$
\gamma= \pm \frac{\underline{x}_{*}^{\top} \underline{G}_{\star}}{\left(\underline{G}_{*}^{\top} \underline{\Lambda} \underline{G}_{\star}\right)^{\frac{13}{2}}} \geq 0
$$


The choice for the sign must be made so that $\gamma \geq 0$.

If $g_{1}(\underline{x})$ is expanded at $\underline{x}=\underline{x}_{*}$ and the higher order terms are disregarded,

$$
g_{\star}(\underline{x})=\underline{x}_{*}^{\top} \underline{G}_{*}
$$

Hence,

$$
\begin{aligned}
& \bar{g}_{\star}=-\underline{x}_{\star}^{\top} \underline{G}_{\star} \\
& \sigma_{g_{\star}}=\left(\underline{G}_{*}^{\top} \underline{\Lambda} \underline{G}_{\star}\right)^{\frac{1}{2}}
\end{aligned}
$$

and therefore $\gamma$ in Eq. 4.50 represents the safety index or its absolute value since

$$
\beta=\frac{\bar{g}_{\star}}{\sigma_{g_{\star}}}=-\frac{\underline{x}_{*}^{\top} \underline{G}_{\star}}{\left(\underline{G}_{\star}^{\top} \underline{\Lambda} \underline{G}_{\star}\right)^{\frac{1 / 2}{2}}}
$$

With respect to the preceding analytical development, the following observations can be made.

(a) For the determination of design point $\underline{x}_{\star}$, an iterative procedure has been proposed by Rackwitz [1976]. The present interpretation, however, indicates that the optimization algorithms currently available for nonlinear programming methods including Lagrange multiplier methods may be used for the same purpose.

(b) If a random vector $\underline{x}$ is Gaussian with uncorrelated (independent) components, the joint density function $f(\underline{x})$ is given by

$$
f(\underline{x})=\frac{1}{(\sqrt{2 \pi})^{n}} \exp \left(-\frac{1}{2} \underline{x}^{\top} \underline{x}\right)
$$


while if $\underline{x}$ is Gaussian with correlated components

$$
f(x)=\frac{1}{(\sqrt{2 \pi})^{n}|\underline{\Lambda}|^{\frac{3}{2}}} \exp \left(-\frac{1}{2} \underline{x}^{\top} \underline{\Lambda}^{-1} \underline{x}\right)
$$

Since $\gamma_{0}$ is defined as $\gamma_{0}=\left(\underline{x}^{\top} \underline{x}\right)^{\frac{3}{2}}$ or $\gamma_{0}=\left(\underline{x}^{\top} \underline{\Lambda}^{-1} \underline{x}\right)^{\frac{3}{2}}$, it follows from Eqs. 4.55 and 4.56 that the design point $\underline{x}_{\star}$ is the point of maximum likelihood (see Figs. 4.3 and 4.4) regardless of the correlations, although the interpretation of $\gamma=|\beta|$ as the shortest distance between the origin and the limit state surface is no longer valid if $\Lambda \neq I$. Even in this case, however, $|\beta|$ represents the shortest distance between the origin and the limit state surface in the $y$ space, since it can be shown that

$$
|\beta|=\left(\underline{y}_{*}^{\top} \underline{y}_{\star}\right)^{\frac{3}{2}}=\left(\underline{x}_{*}^{\top} \underline{\Lambda}^{-1} \underline{x}_{*}\right)^{\frac{1}{2}}
$$

by virtue of Eq. 4.43. Lind [1979] made a comment, among other things, to the same effect, dealing, however, only with the case where $\underline{x}$ is Gaussian with independent components .

If the analytical forms of the limit state surface in the original $x$ space are defined in such a way that one of the components of $\underline{x}\left(\operatorname{say}, x_{i}\right)$ can be expressed in terms of the others,

$$
x_{i}=x_{i}\left(\underline{x}^{\prime}\right)
$$

where $\underline{x}^{\prime}=\left[\begin{array}{lllllll}x_{1} & x_{2} & \ldots & x_{i-1} & x_{i+1} & \ldots & x_{n}\end{array}\right]^{\top}$ and if the random vector $\underline{x}$ is Gaussian, then $\beta^{2}=\underline{x}^{\top} \underline{x}$ or $\underline{x}^{\top} \underline{\Lambda}^{-1} \underline{x}$ can be written as a function of $\underline{x}^{\prime}$ and 
the design point $\underline{x}_{t}^{\prime}$ can be obtained from

$$
\frac{\partial \beta^{2}}{\partial \underline{x}^{\prime}}=0
$$

and $x_{i}^{*}$ from

$$
x_{i}^{*}=x_{i}\left(\underline{x}_{*}^{\prime}\right)
$$

Thus, the optimization problem formulated above becomes a straightforward elementary exercise of finding the minimum of $B$ without constraining conditions. In fact, the example taken from [Fiessler et al, 1979] can be easily solved in this fashion. The limit state equation in this case is given by

$$
g_{1}(\underline{x})=\pi\left(x_{1}-29\right)^{2}\left(x_{2}-170\right) / 875-T=0
$$

Expressing $x_{2}$ in terms of $x_{1}$,

$$
x_{2}=\frac{875 T}{\pi\left(x_{1}-29\right)^{2}}+170
$$

and minimizing $B$,

$$
B=\sqrt{x_{1}^{2}+\left(875 T /\left\{\pi\left(x_{1}-29\right)^{2}\right\}+170\right)^{2}}
$$

The minimum value of $\beta$ and the design point $\underline{x}_{*}$ agree with the corresponding values listed in [Fiessler et al, 1979]. Even the second example in [Fiessler et al, 1979] which involves a random vector $\underline{x}$ with eight (8) independent components can be solved without difficulty using the same procedure, if $x$ is Gaussian. 


\subsection{Dependence of on Distribution Functions}

In the preceding development of mean value and advanced FOSM methods, the reliability index $\beta$ was defined in such a way that it depends only on the means and variances of the basic variables appearing in the limit state equation, but not on the distribution functions of these variables. Under certain conditions, the FOSM method provides the exact solution in the following sense: The design point which determines the $\beta$ value is nothing but the point of maximum likelihood and the $B$ value in turn determines exactly the limit state probability $P_{f}$. The conditions are such that the limit state equation consists only of a linear combination of normal variables. The simplest example is the case where $R$ and $Q$ are normal and statistically independent; then, as indicated by Eqs. 4.5 and 4.11 , the reliability in$\operatorname{dex} B$ is related to the 1 imit state probability $P_{f}$ by

$$
P_{f}=\Phi(-\beta) \quad \text { or } \quad B=\Phi^{-1}\left(1-P_{f}\right)
$$

Even when linearity of the limit state equation cannot be assumed, the equivalence of the design point and the point of maximum likelihood follows from the assumption that the basic variables in the limit state equation are all normally distributed. In actual design situations, however, even the less stringent assumption that the basic variables are all normal (but the limit state equation is not necessarily a linear combination thereof) is not particularly realistic. Therefore, it is highly desirable to develop procedures that can determine the $\beta$ value and that take into account the effects of the non-normality of the variables appearing in the limit state equation. In this respect, the $B$ value derived in the preceding analys is on the basis of the advanced FOSM method (disregarding the nature of the 
distribution functions af the basic variables) is interpreted as its reference value corresponding to the case where all the basic variables are normally distributed. The following paragraphs describe the procedures by which the $B$ value can be determined, still within the framework of the FOSM, while taking into consideration the possible non-normal characteristics of the basic random variables.

For simplicity, statistical independence among the components of $\underline{x}$ is assumed. Then, the non-normal components $x_{i}$ can be transformed into the normal components $u_{i}$ by

$$
u_{i}=\Phi^{-1}\left\{F_{i}\left(x_{i}\right)\right\}
$$

where $F_{j}(\cdot)$ are the (non-normal) distribution functions of $x_{j}$ and $\Phi^{-1}(\cdot)$ is the inverse standardized normal function. Writing $u_{j}$ for the normal components $x_{j}$ as well, the random vector $\underline{x}$ can be transformed into a norma) random vector $\underline{u}$ with all of its components independent. Note that both the $x$ 's and $u$ 's are standardized variables.

The transformation from $\underline{x}$ into $\underline{u}$ requires that the limit state equations $g_{i}(\underline{x})$ be also transformed accordingly with the aid of

$$
x_{i}=F_{i}^{-1}\left\{\phi\left(u_{i}\right)\right\}
$$

or symbolically,

$$
\underline{x}=F^{-1}\{\Phi(\underline{u})\}
$$

Substituting Eq. 4.67 into $g_{1}(\underline{x})$, one obtains the limit state condition in the $\underline{u}$ space: 


$$
g_{1}(\underline{x})=g_{1}\left[F^{-1}\{\Phi(\underline{u})\}\right]=h_{1}(\underline{u}) \leqq 0
$$

Then, the analysis developed in the preceding sections can readily be applied to the random vector $\underline{x}$ with independent but possibly non-normal components (not necessarily all of them) by considering its normal transformation $\underline{u}$ with the independent components under the limit state conditions $h_{1}(\underline{u}) \leq 0$. Thus, the design point $\underline{u}_{*}$ in the $\underline{u}$ space is obtained as the point of maximum likelihood along the constraint $h_{1}(\underline{u})=0$. Transforming $\underline{u}^{\star}$ back into the $\underline{x}$ space by means of Eq. 4.67, one obtains the design point $\underline{x}^{*}=F^{-1}\left\{\Phi\left(\underline{u}^{*}\right)\right\}$. The limit state conditions $g_{1}(\underline{x}) \leq 0$ in the original $\underline{x}$ space is, in most cases, of a relatively simple analytical form. Unfortunately, the transformation of $\underline{x}$ into $\underline{u}$ as described here will usually result in the limit state conditions $h_{j}(\underline{u})$ which are of a more complex analytical form. Indeed, this is the penalty imposed by such a transformation. However, one might still find it worthwhile to pay the penalty in terms of the additional analytical as well as numerical work for the succinctness in the mathematical formulation of the problem and in the interpretation of the solution that can be enjoyed in the transformed $\underline{u}$ space. The limit state probability $P_{f}$ is then obtained as

$$
P_{f}=\int_{D} \underline{f} \underline{u}(\underline{\xi}) d \underline{\xi}
$$

where $D$ represents the doma in $h_{1}(\underline{\xi}) \leq 0$ given by Eq. 4.68 .

Instead of pursuing the approach mentioned above, Rackwitz [1976] proposed in essence that the iterative procedure developed on the basis of Eqs. $4.26-4.28$ be utilized even when dealing with non-normal variables. However, in this case, the reliability index $B$ is to be determined in the 
coordinate space of the new set of "equivalent normal" variables standardized with the mean $\bar{X}_{i}^{N}$ and standard deviation $\sigma_{i}^{N}$

$$
\begin{aligned}
& \bar{x}_{i}^{N}=x_{i}^{\star}-\Phi^{-1}\left[F_{i}\left(x_{i}^{*}\right)\right] \sigma_{i}^{N} \\
& \sigma_{i}^{N}=\frac{\phi\left\{\Phi^{-1}\left[F_{i}\left(x_{i}^{*}\right)\right]\right\}}{f_{i}\left(x_{i}^{\star}\right)}
\end{aligned}
$$

where $\phi(\cdot)$ indicates the standardized normal density function and $x_{i}^{*}$ represents the $i-t h$ component of the design point $\underline{x}_{*}$ (in the original variable space) obtained at the end of an iterative cycle. Eqs. 4.70 and 4.71 follow from the stipulation that the (non-normal) distribution function $F_{j}(x)$ and density function $f_{j}(x)$ of $x_{j}$ are equal, respectively, to $\Phi(x)$ and $\phi(x)$ at $x=x_{i}^{*}$. When the $B$ values computed using this approximate technique are combined with Eq. 4.64, the agreement with $P_{f}$ computed exactly from Eq. 4.3 is often excellent. However, this procedure does not reduce the error which is due to the linearization of what is generally a nonlinear limit state equation at the design point. In situations where this is a significant problem, an improvement may be obtained by approximating the limit state equation by a quadratic, rather than 1 inear, function [Fiessier et al, 1979].

\subsection{Design Point as the Point of Maximum Likelihood}

The interpretation of the design point (at which $B^{2}=\underline{x}^{\top} \underline{x}$ or $\underline{x}^{\top} \underline{\Lambda}^{-1} \underline{x}$ is minimized) as the point of maximum likelihood is valid only when $\underline{x}$ is normal, although it is noted that even if $\underline{x}$ is not normal, the minimum value of $\beta^{2}=\underline{x}^{\top} \underline{x}$ or $\underline{x}^{\top} \underline{\Lambda}^{-1} \underline{x}$ does serve as the safety index $B=\bar{g}_{*} / \sigma_{g_{*}}$ (see 
Eqs. 4.41 and 4.54$)$. It then appears reasonable and also useful if the design point is defined as the point of maximum likelihood, regardless of which distribution the random vector $\underline{x}$ obeys. Such a definition of the design point and the corresponding definition of load factors were first given by A.M. Freudenthal and M. Shinozuka [1961] in one of their early studies on structural reliability.

In order to examine the validity of such a statement, consider the following example, studied by Ellingwood et al [1980]. The limit state equation is given by

$$
g(X, Z)=X Z-M \leqq 0
$$

where $X$ is log-normally distributed and represents the yield strength while $Z$, independent of $X$ and normally distributed, represents the section modulus. Eq. 4.72 indicates that if the resisting bending moment $X Z$ is less than or equal to the applied (deterministic) bending moment $M$, then failure will occur. It is assumed that $\mu_{X}=38 \mathrm{ksi}, \sigma_{X}=3.8 \mathrm{ksi}, \mu_{Z}=54 \mathrm{in}^{3}, \sigma_{Z}=$ 2.7 in $^{3}$ and $M=1140 \mathrm{kip}-\mathrm{in}$, where $\mu$ and $\sigma$ denote, respectively, the mean value and standard deviation of the subscripted variables. The nondimensionalized form of the 1 imit state equation can be obtained by dividing both sides of Eq. 4.72 by $\sigma_{x}{ }^{\sigma} Z^{:}$

$$
g(X, Z)=\frac{x}{\sigma_{X}} \cdot \frac{z}{\sigma_{Z}}-\frac{M}{\sigma_{X} \sigma_{Z}}=0
$$

If the corresponding lower-case letters are used for the standardized versions of these random variables, the limit state equation in Eq. $4.73 \mathrm{can}$ be transformed into (see Fig. 4.5) 


$$
g_{z}(x, z)=\left(x+\mu_{x} / \sigma_{x}\right)\left(z+\mu_{z} / \sigma_{z}\right)-m=0
$$

where $m=M /\left(\sigma_{X} \sigma_{Z}\right)$. Since $X$ is log-normally distributed, $U=\ln \left(X / \sigma_{X}\right)$ is normally distributed with $\mu_{U}=2.30$ and $\sigma_{U}=0.0998$. This relationship can be rewritten as

$$
\sigma_{U} u+\mu_{U}=\ln \left(x+\mu_{x} / \sigma_{X}\right)
$$

Then, substituting Eq. 4.75 into Eq. 4.74 one obtains,

$$
e^{\sigma^{U} u+\mu_{U}}\left(z+\mu_{Z} / \sigma_{Z}\right)-m=0 \quad \text { or } \quad z=m e^{-\left(\sigma_{U} u+\mu_{U}\right)}-\mu_{Z} / \sigma_{Z}
$$

Since $u$ and $z$ are standardized normal variables, the safety index $B$ is obtained, assuming that $\beta>0$, as the minimum of

$$
B^{2}=u^{2}+z^{2}
$$

under the constraining condition given by Eq. 4.76. This is equivalent to finding the minimum value of $\beta^{2}$;

$$
B^{2}=u^{2}+z^{2}=u^{2}+\left\{m e^{-\left(\sigma_{u} u+\mu_{u}\right)}-\mu_{z} / \sigma_{z}\right\}^{2}
$$

Hence, the (exact location of the) design point $\left(u^{*}, z^{*}\right)$ is determined first by solving for $u=u^{*}$ the following transcendental equation obtained from $d B^{2} / d u=0$ :

$$
u-m \sigma_{U} e^{-\left(\sigma_{U} u+\mu_{U}\right)}\left\{m e^{-\left(\sigma_{U} u+\mu_{u}\right)}-\mu_{Z} / \sigma_{Z}\right\}=0
$$

and then $z=z^{*}$ is obtained from Eq. 4.76. The numerical result indicates that $u^{*}=-4.465, z^{*}=-2.568$ and $B=5.151$, where the assumption that 
$B>0$ is verified since $\underline{x}_{\star} \underline{G}_{\star}=x^{*}\left(z^{*}+\mu_{Z} / \sigma_{Z}\right)+u^{*}\left(z^{*}+\mu_{Z} / \sigma_{Z}\right)<0$ in this case.

The exact procedure for locating the design point and evaluating the safety index has been easily implemented above mainly because it is possible to transform the non-normal variable $x$ into the standardized normal variable $u$ in a simple analytical form (Eq. 4.75) which in turn aided the establishment of a simple analytical relationship between $u$ and $z$ (Eq. 4.76). As mentioned earlier, however, this is the exception rather than the rule. In the following, therefore, a method is described by which the design point and safety index are evaluated in approximation. Indeed, this is the method in which the point of maximum likelihood in the original $\underline{x}$ space is defined as the design point $\underline{x}_{*}$, regardless of which distribution $\underline{x}$ obeys. The safety index $\beta$ is then obtained as $B= \pm\left(\underline{u}_{*}^{\top} \underline{u}_{*}\right)^{\frac{1}{2}}$ where $\underline{u}_{*}$ is a point in the $\underline{u}$ space corresponding to $\underline{x}_{\star}$, through the normal transformation. For this purpose, the limit state surface (Eq. 4.74) is used. The density function $f_{x}(x)$ of $x$ and $f_{z}(z)$ of $z$ can be shown to be $f_{x}(x)=\frac{1}{\sqrt{2 \pi} \sigma_{U}} \exp \left\{-\ln \left(x+\mu_{X} / \sigma_{X}\right)-\left[\ln \left(x+\mu_{X} / \sigma_{X}\right)-\mu_{U}\right]^{2} /\left(2 \sigma_{U}^{2}\right)\right\} x>0$ $=0$ otherwise and

$$
f_{z}(z)=\frac{1}{\sqrt{2 \pi}} e^{-z^{2} / 2}
$$

Since the joint density function of $x$ and $z$ is given by $f_{x}(x) f_{z}(z)$, the point $\left(x^{*}, z^{*}\right)$ of maximum likelihood is obtained by minimizing $\gamma_{0}^{2}$ : 


$$
\gamma_{0}^{2}=2 \ln \left(x+\mu_{x} / \sigma_{x}\right)+\left[\ln \left(x+\mu_{x} / \sigma_{X}\right)-\mu_{U}\right]^{2} / \sigma_{U}^{2}+z^{2}
$$

Solving Eq. 4.74 for z, substituting into Eq. 4.82 and setting $\mathrm{dr}_{0}^{2} / \mathrm{dx}=0$, one obtains

$$
\begin{gathered}
\left(x+\mu_{x} / \sigma_{x}\right)+\left(x+\mu_{x} / \sigma_{x}\right)\left[\ln \left(x+\mu_{x} / \sigma_{X}\right)-\mu_{U}\right] / \sigma_{U}^{2} \\
-m\left\{\frac{m}{\left(x+{ }_{\mu_{X}} / \sigma_{X}\right)}-\frac{\mu_{Z}}{\sigma_{Z}}\right\}=0
\end{gathered}
$$

The root of Eq. 4.83 is found to be $x^{*}=-3.639$. Upon substituting this value into Eqs. 4.74 and $4.75, z^{*}=-2.530$ and $u^{*}=-4.489$ are obtained, respectively. The safety index is then computed as $B=\sqrt{(-4.489)^{2}+}$ ${\overline{(-2.530)^{2}}}^{2}=5.153$. While these values are approximations to the exact values $u^{\star}=-4.465, z^{\star}=-2.568$ and $\beta=5.151$ obtained previously, their agreements are extremely good. Indeed, if the variable $x$ in Eq. 4.83 is transformed into $u$ by virtue of Eq. 4.75 then Eq. 4.83 becomes

$$
\sigma_{U}+u-m \sigma_{U} e^{-\left(\sigma_{U} u+\mu_{U}\right)}\left\{m e^{-\left(\sigma_{U} u+\mu_{U}\right)}-\mu_{Z} / \sigma_{Z}\right\}=0
$$

which is obviously not identical with Eq. 4.79. That $\sigma_{U}=0.0998$ is small compared with the absolute values of the root of Eq. 4.79 as well as that of Eq. 4.84 has contributed to the fact that these two roots are practicalIy identical.

The iterative procedure by Rackwitz [1976] applied to this problem produces $x^{*}=-3.629, z^{*}=-2.552$ and $B=5.144$. These values and the results of the exact as well as approximate analyses carried out in the present study are summarized in Table 4.1 for easy comparison. While the 
kind of excellent agreement observed in Table 4.1 between the exact and the approximate solutions cannot be guaranteed for other cases, particularly those involving a larger number of non-normal variables, it is expected that the proposed approximate method will serve as a practical alternative to the exact method which is articulated here and to the Rackwitz iterative procedure.

\subsection{Estimation of Limit State Probabilities}

In certain cases, it may be desirable or necessary to estimate the limit state probabilities directly. Therefore, without loss of generality, consider that the resistance and load variables $\underline{x}$ are standardized and uncorrelated and that the limit state equation is given by $g_{1}(\underline{x}) \leq 0$. Then, similarly to Eq. 4.3, the limit state probability $P_{f}$ can be evaluated from

$$
P_{f}=\int_{D} f_{\underline{x}}(\underline{\xi}) d \underline{\xi}
$$

where $D$ indicates the doma in where $g_{1}(\underline{x}) \leq 0$ and $f_{\underline{x}}(\underline{\xi})=f_{x_{1} x_{2} \ldots x_{n}}\left(\xi_{1}, \xi_{2}\right.$, $\ldots, \xi_{n}$ ) the joint density function of $\underline{x}$. For a well-behaved concave (towards the origin) 1 imit state surface, the domain of integration is schematically given by $D=D_{1} \cup D_{2}$ in Fig. 4.3 (where the contour lines for the joint density are generally not concentric circles if $\underline{x}$ is not normal, however). Under the further assumption that $\underline{x}$ is normal, it can be shown that

$$
1-x_{n}^{2}\left(\beta^{2}\right) \geq P_{f} \geq 1-\Phi(\beta)
$$

The first member of Eq. 4.86 represents the integral of the joint density 
function over the domain outside the circle $C_{0}$ (Fig. 4.3) that passes the design point and therefore is an upper bound of $P_{f}$. The function $x_{n}^{2}(\cdot)$ indicates the $x^{2}$ distribution with $n$ degrees of freedom according to which $B^{2}=\underline{x}^{\top} \underline{x}$ distributes. This upper bound is apparently due originally to A.M. Hasofer (unpublished). The third member of Eq. 4.86 is a lower bound since it represents the integral of the joint density function over the doma in $D_{1}$ bounded by the linearized limit state surface $g_{*}(\underline{x})=0$. Unfortunately, these bounds are often not particularly close to each other.

An alternative approach then is to perform the integration shown in Eq. 4.85 by the Monte Carlo method. In fact, the straightforward Monte Carlo method can easily be implemented and $P_{f}$ estimated as

$$
P_{f}=\frac{A}{N} \sum_{i=1}^{N} \frac{\delta_{i}}{(2 \pi)^{n / 2}} \exp \left(-\frac{1}{2} \underline{n}_{i}^{\top} \underline{n}_{i}\right)
$$

with $N$ being equal to the sample size and

$$
\delta_{i}= \begin{cases}0 & \text { if } g\left(\underline{n}_{i}\right)>0 \\ 1 & \text { if } g\left(\underline{n}_{i}\right) \leq 0\end{cases}
$$

where $\underline{n}_{i}$ is the $i$-th realization of $\underline{x}$ generated by means of a computer simulation of the $n$ independent, uniformly distributed random variables over an appropriate multidimensional square or rectangular domain whose area is A. The appropriate shape and size to be considered for such a domain depend on the location of the design point as well as the goemetry of the limit state surface relative to the design point. It is rather obvious that the domain should contain a region of high likelihood around the de- 
sign point.

A numerical example taken from Fiessler et al [1979] is used to demonstrate the validity of the Monte Carlo integration method with the limit state surface given by Eq. 4.61. The design points and the exact values of $P_{f}$ listed in Fiessler et al [1979] are reproduced in Table 4.2. The Monte Cario evaluation of $P_{f}$ is performed using the rectangular domains $A$ of integration, as indicated in Table 4.2, that are assigned in an ad hoc fashion (except to insure that these domains contain the design points and the regions of high likelihood). The resulting value of $P_{f}$ for each value of $T$, based on one thousand simulated points within the domain $A$, is also listed in Table 4.2 in parentheses. The agreement between the exact and the Monte Carlo solutions is extremely good. The computer time required for the present example is a fraction of a second including the time for the various overhead operations even on an HP minicomputer system (CPU: 21 MXE).

It is acknowledged that the above observation is based on the result of a Monte Carlo integration method applied to a relatively simple numerical example. However, it should still be emphasized that the Monte Carlo method is a powerful practical tool, if employed intelligently, in spite of the understandable reluctance to use it, particularly on the part of academicians, because of its lack of mathematical novelty and attractiveness. When the problem becomes more complicated (involving a larger number of variables), use of the Monte Carlo method is expected to become expensive. However, the exact solution would be even more expensive to obtain, except possibly for those unusual cases where the limit state surface has a special form amenable either to a closed form analytical integration or to a cost-wise tract- 
able numerical integration. Therefore, the Monte Carlo method seems to provide the only practical means for obtaining the solution under general conditions at this time. In fact, Fiessler et al [1979] use the Monte Carlo solution in lieu of the exact solution when evaluating the accuracy of the various approximate solutions dealing with an example involving eight (8) random variables. Obviously, when the problem complexity increases; the use of a more efficient and sophisticated Monte Carlo method of integration such as the "importance sampling technique" should be considered.

Another interesting approach is to use the Stokes and Gauss divergence theorem, for, respectively, two (2)- and three (3)-dimensional problems. The approach appears to possess the potential to be expanded and used for multidimensional problems. Consider, for example, that the random vector $\underline{x}=\left[\begin{array}{ll}x_{1} & x_{2}\end{array}\right]^{\top}$ is normal with $x_{1}$ and $x_{2}$ being independent of each other, with the density function

$$
f_{\underline{x}}\left(x_{1}, x_{2}\right)=\frac{1}{2 \pi} \exp \left\{-\frac{1}{2}\left(x_{1}^{2}+x_{2}^{2}\right)\right\}
$$

which can be rewritten as

$$
\underline{f}_{\underline{x}}\left(x_{1}, x_{2}\right)=\frac{1}{2}\left[\frac{\partial}{\partial x_{1}}\left\{\phi\left(x_{1}\right)_{\phi}\left(x_{2}\right)\right\}+\frac{\partial}{\partial x_{2}}\left\{\phi\left(x_{1}\right) \phi\left(x_{2}\right)\right\}\right]
$$

where $\phi\left(x_{i}\right)$ is the density function of $x_{i}$. Then, with the aid of the Stokes theorem, it can be shown that the area integral producing the limit state probability $P_{f}$ given by $E q .4 .85$ reduces to a contour integral:

$$
P_{f}=\iint_{D} f_{\underline{x}}\left(x_{1}, x_{2}\right) d x_{1} d x_{2}=\frac{3}{2} \oint_{C}\left\{\Phi\left(x_{1}\right) \phi\left(x_{2}\right) l_{1}+\phi\left(x_{1}\right) \Phi\left(x_{2}\right) \ell_{2}\right\} d s
$$

where, referring to Fig. 4.6, C is the closed path of contour integration 
around the domain $D$ bounded by $g_{1}(\underline{x})=0$ and its infinite extension indicated by the dashed line, and $\ell_{i}(i=1,2)$ are the directional cosines of the outward normal $n$ along the integration path $C$. In view of the fact that the density function given by Eq. 4.89 decays rapidly as a negative exponential function of the square of the distance from the origin, the limit state probability $P_{f}$ can be evaluated in approximation by carrying out the contour integral numerically over a relatively short stretch of the integration path C containing the design point $\underline{x}_{\star}$. For this purpose, the numerical values of $\phi(x)$, should be stored in the computer in a tabulated form (or any other convenient way) to be used with an interpolation scheme. For larger values of $x$ (say $\geq 4.0$ ), the following asymptotic expansion may be conveniently used:

$$
\Phi(-x)=\frac{1}{\sqrt{2 \pi} x} e^{-x^{2} / 2}\left(1-\frac{1}{x^{2}}+\frac{3}{x^{4}}-\cdots\right)
$$

If the random vector is $\underline{x}=\left[\begin{array}{lll}x_{1} & x_{2} & x_{3}\end{array}\right]^{\top}$ with independent components, the joint density can be written as

$$
\begin{aligned}
f_{\underline{x}}\left(x_{1}, x_{2}, x_{3}\right) & =\frac{1}{3}\left[\frac{\partial}{\partial x_{1}}\left\{\phi\left(x_{1}\right) \phi\left(x_{2}\right) \phi\left(x_{3}\right)\right\}+\right. \\
& \left.+\frac{\partial}{\partial x_{2}}\left\{\phi\left(x_{1}\right) \Phi\left(x_{2}\right) \phi\left(x_{3}\right)\right\}+\frac{\partial}{\partial x_{3}}\left\{\phi\left(x_{1}\right)_{\phi}\left(x_{2}\right) \Phi\left(x_{3}\right)\right\}\right]
\end{aligned}
$$

When this expression is used in Eq. 4.85 and the Gauss divergence theorem is thereupon applied, the following integral expression results for the limit state probability $P_{f}$ : 


$$
\begin{aligned}
P_{f} & =\iiint_{D} f_{\underline{x}}\left(x_{1}, x_{2}, x_{3}\right) d x_{1} d x_{2} d x_{3} \\
& =\frac{1}{3} \iint_{S}\left\{\Phi\left(x_{1}\right)_{\phi}\left(x_{2}\right) \phi\left(x_{3}\right) e_{1}+\right. \\
& \left.+\phi\left(x_{1}\right) \Phi\left(x_{2}\right) \phi\left(x_{3}\right) e_{2}+\phi\left(x_{1}\right) \phi\left(x_{2}\right) \Phi\left(x_{3}\right) e_{3}\right\} d S
\end{aligned}
$$

where $S$ is the surface boundary of the three-dimensional doma in $D$ bounded by the limit state surface $g_{1}(\underline{x})=0$ and $i t s$ infinite extension, and $\ell_{i}(i=1,2,3)$ are the directional cosines of the outward normal $\underline{n}$ over the surface $S$. As in the two-dimensional case, the surface integral can be performed only over a relatively small area containing the design point $\underline{x}_{\star}$ for an approximate estimation of the limit state probability $P_{f}$.

The reduction in the multiplicity of the integration by one, as observed in the examples just mentioned, may represent considerable savings in computational effort when the multiplicity of the original integral is as small as two (Eq. 4.91) or three (Eq. 4.94). More important, however, is the fact that the preceding approach provides an alternate integral expression for the limit state probability, which may be thus evaluated more easily. An extension of this approach to general multidimensional cases is an interesting subject for future study.

If a limit state has to be defined by $n$ limit state equations $(n \geq 2)$, e.g., representing $n$ ultimate structural failure modes,

$$
g_{i}(x) \leq 0 \quad(i=1,2, \ldots, n)
$$

the limit state probability $P_{f}$ is given by Eq. 4.85 with $D$ indicating the domain where all the constraints specified by Eq. 4.95 are observed (see Fig. 4.7). The evaluation of $P_{f}$ can then proceed, for example, with the aid of 
the Monte Carlo method just mentioned: Ordinarily, the increased complexity of the problem, due to the added constraining conditions, has only a slight, if any, impact on the computational cost. Under these multiple limit state conditions, however, the FOSM approach in which a single safety index is used as a measure for the limit state probabability does not usually apply. Indeed, it is for this reason that, under the aforementioned conditions, the safety index must be relegated to a "baseline estimate" of $P_{f}$ obtained with the aid of a "specific joint density" assumed for the random vector $\underline{x}$, with the difficulty indicated in the Introduction notwithstanding. If however, the safety index approach is insisted upon, it may be defined as

$$
B=-\Phi^{-1}\left(P_{f}\right)
$$

where $P_{f}$ is a baseline estimate of $P_{f}$ under the assumption, for example, that the random vector $\underline{x}$ is normal with independent components. This definition of the safety index is more general in the sense that the $B$ introduced in Eq. 4.24 or Eq. 4.41 is associated with only one limit state surface (while in reality a limit state, e.g., the state of ultimate structural failure, is usually defined by more than one limit state equation). The following inequality may be useful for the limit state probability

$$
\Phi\left(B_{m}\right) \leqq P_{f} \leqq \sum_{i=1}^{n} \Phi\left(-B_{i}\right)
$$

where $B_{\mathbf{i}}$ is the safety index associated with the limit state surface $\mathbf{g}_{\mathbf{j}}(\underline{x})=$ 0 as defined by Eq. 4.24 or Eq. 4.41, and the subscript $m$ is such that $\Phi\left(B_{m}\right)$ $\geq \Phi\left(B_{i}\right)$ for $i=1,2, \ldots, n$. A sufficient condition under which Eq. 4.97 is valid is: (1) All limit state surfaces are concave with respect to the origin $\underline{x}=0$ and (2) $B_{i}$ are positive. 
4.7 Second Order Higher Moment Methods and General Forms of Probability Distribution and Density Functions

\subsubsection{Second Order Higher Moment Methods}

In the advanced FOSM methods, the limit state function $g_{1}(\underline{x})$ is expanded into a Taylor series around the design point $\underline{x}_{*}$, and its linearized version $g_{0}(\underline{x})$ is used in deriving the reliability index $B$ (Eq. 4.41 or Eq. 4.54). If $D_{1}$ denotes the domain defined by $g_{0}(\underline{x}) \leq 0$, then the first order approximation $P_{f 1}$ for the limit state probability $P_{f}$ can be obtained from the integration given by Eq. 4.85 with the domain $D$ replaced by $D_{1}$. The result of this integration is (see also Eq. 4.86)

$$
P_{f 1}=1-\Phi(\beta)
$$

under the assumption that $\underline{x}$ is a normal vector. This assumption is not unreasonable, since the original basic variables $\underline{X}$ in Eq. 4.1 , even if they are non-normal, can be transformed into normal variables $\underline{u}$ by means of Eq. 4.65 (rewriting $\underline{x}$ for $\underline{u}$ ) as long as $\underline{x}$ are component-wise independent.

If, however, the Taylor series is truncated after the third term, rather than the second, one obtains the second order approximation $g_{00}(\underline{x})$ of $g_{1}(\underline{x})$

$$
g_{00}(\underline{x})=\sum_{i=1}^{n} \Delta x_{i}^{*}\left(\frac{\partial g_{1}}{\partial x_{i}}\right)+\frac{1}{2} \sum_{i=1}^{n} \sum_{j=1}^{n} \Delta x_{i}^{*} \Delta x_{j}^{*}\left(\frac{\partial^{2} g_{1}}{\partial x_{j} \partial x_{j}}\right)
$$

The second order approximation for the limit state condition is then given by $g_{00}(\underline{x}) \leq 0$. If $D_{2}$ denotes the doma in of $\underline{x}$ defined by $g_{00}(\underline{x}) \leq 0$, then the corresponding approximate limit state probability $P_{f 2}$ can again be given by Eq. 4.85, with $D$ replaced by $D_{2}$. Indeed, Fiessler et al, [1979] reported that some improvement is achievable in the estimation of the limit state 
probability essentially by means of this second order approximation. Obviously, the extent of the improvement represented by $P_{f 2}$ over $P_{f 1}$ depends on how much closer the form of $g_{1}(\underline{x})$ can be approximated by the general quadratic form of Eq. 4.99 than by the linear form of Eq. 4.18.

It is also possible to construct the reliability index $\beta$ on the basis of the second order approximation:

$$
B=\frac{\bar{g}_{00}}{\sigma_{g_{00}}}
$$

where $\bar{g}_{00}$ and $\sigma_{g_{00}}$ are the mean value and standard deviationof $g_{00}(\underline{x})$, respectively. The mean value $\bar{g}_{00}$ can be obtained with relative ease as

$$
\bar{g}_{00}=-\sum_{i=1}^{n} x_{i}^{*}\left(\frac{\partial g_{1}}{\partial x_{i}}\right)+\frac{1}{2} \sum_{i=1}^{n} \sum_{j=1}^{n}\left(\rho_{i j}+x_{i}^{*} x_{j}^{*}\right)\left(\frac{\partial^{2} g_{1}}{\partial x_{i} \partial x_{j}}\right)
$$

where $\rho_{i j}$ is such that

$$
\rho_{i j}=E\left[x_{i} \quad x_{j}\right]
$$

The expression for the standard deviation $\sigma_{g_{00}}$ is not only lengthy but also involves the moments of $\underline{x}$ around $\underline{x}^{\star}$ up to the fourth order. Since the moments of such higher orders are known to be statistically unstable, the statistical confidence of $\sigma_{g_{00}}$ involving such higher order moments is not particularly high. The loss of geometrical significance and mathematical simplicity that accompany the reliability index $\beta$ based on the FOSM is also difficult to disregard. However, more important is the fact that it is not quite clear at this time whether or not, and to what extent, the use of the index $B$ based on the second order approximation, rather than the first order, improves the state of the art of structural reliability analysis. For these reasons, 
the explicit expression for $\sigma_{g_{00}}$ and hence that for $\beta$ in Eq. 4.100 are not given here. Further discussion on this subject is presented in Appendix $D$, however.

\subsubsection{General Forms of Probability Distribution and Density Functions}

The distribution functions, other than normal and log-normal, that are often used for the basic variables in the limit state equation, include the beta, gamma (exponential as its special case) and asymptotic extremal (Weibul1, Gumbel, Frechet, etc.) distributions. There are, however, a large number of other distribution functions that are potentially useful for structural reliability analysis. In this respect, Shinozuka [1979] made a study on the possible engineering applications of a rather exhaustive set of distribution functions such as those derivable from the Johnson and Pearson distribution families (to which, incidentally, the uniform, beta, gamma, normal and log-normal distributions belong).

It is also possible to express a probability density function in terms of a series expansion whose coefficients involve the moments of the random variable $X$. Typical examples are the Gram-Charlier series of Types $A$ and $C$, the series derived from the gamma distribution function and the asymptotic series. The asymptotic series was developed in a paper by Shinozuka and $\mathrm{Ni}$ shimura [1965]. In the same paper, they examined the applicability of these series to a structural reliability analysis problem and concluded that the combined use of the Gram-Charlier series of Type A for small to moderate values of $X$ and the asymptotic series for large values of $|X|$ would provide a reasonable representation of a density function. An obvious implication of this is that the asymptotic series may be quite useful in structural relia- 
bility analysis where the behavior of the density function for large values of $|X|$ plays a significant role.

The Gram-Charlier series of Type A for a density function $f_{X}(x)$ can be written as

$$
f_{x}(x)=\phi(x)+\frac{C_{3}}{3 !} \phi^{(3)}(x)+\frac{C_{4}}{4 !} \phi^{(4)}(x)+\frac{C_{5}}{5 !} \phi^{(5)}(x)+\cdots
$$

with

$$
c_{3}=-\mu_{3} / \sigma^{3}, C_{4}=\mu_{4} / \sigma^{4}-3, C_{5}=-\mu_{5} / \sigma^{5}+10 \mu_{3} / \sigma^{3}, \cdots
$$

while the asymptotic series can be given by

$$
f_{x}(x) \sim \phi(x)\left(1+\frac{c_{3}^{\prime}}{x^{3}}+\frac{c_{4}^{\prime}}{x^{4}}+\frac{c_{5}^{\prime}}{x^{5}}+\cdots\right)
$$

with

$$
c_{3}^{\prime}=-c_{3}, \quad c_{4}^{\prime}=c_{4}-9 c_{3}, \quad c_{5}^{\prime}=-c_{5}+14 c_{4}-45 c_{3}
$$

where $\phi(x)=$ standardized normal density, $\phi^{(n)}(x)=d^{n} \phi(x) / d x^{n}, \quad \sigma=$ the standard deviation and $\mu_{v}=$ the $v$-th moment about the expected value (hence $\sigma=$ $\sqrt{\mu_{2}}$ ). Further details of the Gram-Charlier and asymptotic series are presented in Appendix E. In connection with such use of the moments $\mu_{v}$ in the density function representation and also in relation to the FOSM methods, the Chebycheff type bounds for the probability that the random variable $X$ (or $|x|$ ) will exceed a certain specified value $x$ may be useful in structural reliability analysis in spite of the fact that these bounds tend to over-estimate the probability, primarily because of its non-parametric nature. For this reason, a list of these bounds is presented in Appendix $F$. 


\subsection{Load Combinations}

\subsubsection{Introduction}

Most structural loads acting on nuclear structures vary with time. If a structure is subjected to only one time-varying load in addition to the dead load, its lifetime structural reliability can be determined by comparing the structural resisting capacity with the sum of the dead load effect and the maximum effect the time-varying load produces during the life of the structure. Since, in general, the dead load varies vary little and slowly, the reliability analys is that is required in this case is relatively simple. However, nuclear structures are acted on by more than one timevarying load during the service life. Then, the maximum effect resulting from the combination of these loads must be compared with the resisting capacity in order to determine the structural reliability. Due to the spatial as well as temporal stochastic nature of these loads, the estimation of such a maximum effect during the structural life and the ensuing reliability analys is is a much more involved task.

The reliability analysis of a structure subjected to a number of stochastic loads is usually performed under the assumptions that (1) the load effects can be combined linearly and (2) the effects of those phenomena such as fatigue, creep, corrosion, etc. that are load-history dependent, can be disregarded. The latter assumption al so implies that the structural resisting capacity is time-invariant. In this contect, the study of load combinations is currently at its inception and has not yet fully developed practical techniques which account for nonlinear load effect combinations and time-dependent phenomena. Therefore, the same assumptions will prevail throughout this study. 
.. unless otherwise stated.

Loads acting on structures can generaliy be classified into load process models such as those shown in Fig. 4.8 [Ellingwood et al, 1980]. Permanent loads $x_{1}(t)$, Fig. 4.8a, such as dead loads maintain a constant magnitude in approximation with a relatively small and slow random variation. Sustained loads $X_{2}(t)$, Fig. 4.8b, may change their magnitude in a stepwise fashion from time to time but in between these changes ramain approximately constant, except for possible small random variations. They may be absent entirely for certain periods. Some of the plant operating loads would fall in this category. Other operating loads, however, do not even have the segment-wise stationarity implied by Fig. $4.8 \mathrm{~b}$. Even then, it is suggested that these loads be modeled in approximation in terms of the segment-wise stationary process depicted by Fig. $4.8 b$ for analytical tractability. Finally, transient loads. $x_{3}(t)$, Fig. $4.8 \mathrm{c}$, are those that occur relatively infrequently and last for a short duration. In fact, their durations are so small relative to permanent and sustained loads that they can be modeled as impulses. Extreme wind and earthquake loads and abnormal loads are examples of such short duration loads. The probability densities for these load processes sampled at any arbitrary point in time are shown at the left hand side of each sample function. The discrete probability mass at zero magnitude indicated by an impulse represents the probability that the load is absent (not acting) at the time of sampling.

When more than one time-varying load is acting, it is unlikely that each load will reach its peak lifetime value at the same moment. This is illustrated in Fig. 4.8d, where, for simplicity, the small random variations the permanent and sustained loads exhibit are disregarded and all the transient loads are approximated by impulse functions. Consequently, a 
structure may be designed for a magnitude of load which is less than the sum of the lifetime peak loads. Indeed, existing standards recognize this; e.g., see Section 4.2 of the ANSI Standard A58.1-1972 and Eq. 9.2 of the ACI Standard 318. These documents have evolved on the basis of past experience rather than a thorough consideration of the underlying stochastic nature of the loads, however.

\subsubsection{Combinations of Random Load Effects}

In what follows, (a) rather than dealing with loads $x_{j}(t)$ directly, the corresponding load effects $Y_{j}(t)$ are considered for the purpose of structural reliability analysis under multiple time-varying loading conditions, (b) these load effects are assumed to be linearly related to the corresponding loads, and represent stresses, strains, displacements, internal forces, etc., and (c) the limit state equation is written in terms of the load effects (instead of the loads themselves). It is reasonable to assume, however, that the temporal nature of the load effects are similar to that of the corresponding loads. Therefore, the load effects $Y_{i}(t)$ can can also be classified into the same three basic categories as shown in Fig. 4.8. Then, what is needed for a rigorous reliability analysis is the probability distribution function of the lifetime maximum $Q=Q_{T}$ of the $\operatorname{sum} Y(t)$

$$
\begin{aligned}
Q=Q_{T}= & \max Y(t) \\
& 0 \leq t \leq T
\end{aligned}
$$

with

$$
Y(t)=Y_{1}(t)+Y_{2}(t)+\ldots+Y_{N}(t)
$$


where $T$ indicates the life of the structure and $N$ the total number of load effects to be combined.

Once the distribution function of $Q$ (which is now a random variable rather than a random process) is found, the probability of failure can be computed from Eq. 4.4, provided, of course, that $R$ is statistically independent of $Q$ and represents the structural resisting capacity, while the safety index $\beta$ can be computed either from Eq. 4.11 or from the advanced FOSM method, accounting for the generally non-normal natures of $R$ and $Q$. Obviously, the validity of this statement is limited to the case where the structural failure can be defined by the simplest limit state equation

$$
g(R, Q)=R-Q \leqq 0
$$

It appears, however, that most structural reliability analyses involving time-varying loads are currently performed for such a class of simple limit state equations.

For the purpose of illustration, consider the special case in which $Y(t)$ consists of $Y_{1}(t)$ and $Y_{2}(t) ; Y(t)=Y_{1}(t)+Y_{2}(t)$. Assume that $Y_{1}(t)$ represents a permanent load effect such as that due to a dead load that can be approximated as a random variable $Y_{1}$, while $Y_{2}(t)$ represents an environmental load effect due, for example, to a wind or earthquake load. For such an environmental load, it is not particularly difficult to estabIish the probability distribution function of the maximum $Q_{2 T}$ of $Y_{2}(t)$ during the lifetime (say $T=50$ years) of the structure [e.g., Ellingwood et al, 1980]. The lifetime maximum $Q=Q_{T}$ of the total load effect $Y(t)$ can then be written as $Q=Y_{1}+Q_{2 T}$. Substituting this into the 1 imit state equation (Eq. 4.109), one obtains $g(R, Q)=R-\left(Y_{1}+Q_{2 T}\right) \leq 0$ in terms of 
the random variables $R, Y_{1}$ and $Q_{2 T}$. The safety index $B$ can then be obtained from the FOSM methods, while the probability of failure can be computed from Eq. 4.4 if the density function $f_{Q}(x)$ of $Q=\gamma_{1}+Q_{2 T}$ can be evaluated. Indeed, the safety index $B$ was evaluated by Ellingrood et al [1980] in this way when the lifetime maximum effect $Q_{2 T}$ of the wind load is combined only with the dead load effect $\gamma_{1}$ under the limiting condition of the live load effect being negligibly small compared with the dead load effect.

Similarly, if $Y(t)$ is given by Eq. 4.108 with $N \geqq 3$, and if $Y_{i}(t)=Y_{i}$ may be considered as random variables except for, say, $Y_{N}(t)$, one obtains the limit state equation $g(R, Q)=R-\left(Y_{1}+Y_{2}+\ldots+Q_{N T}\right)$ in terms of $(N+1)$ random variables, where $Q_{N T}$ is the lifetime maximum of the $N$-th load effect $Y_{N}(t)$. Again, the safety index $B$ can be obtained with the aid of the FOSM methods, while the probability of failure from Eq. 4.4, provided that the density function $f_{Q}(x)$ of $Q=Y_{1}+Y_{2}+\ldots+Q_{N T}$ can be obtained. In fact, if one of the $Y^{\prime} s$, say $Y_{i}(t)$, is the only time-varying load effect, then a $l$ imit state equation of the nonl inear form $g\left(R, Y_{1}, Y_{2}, \ldots, Q_{i T}, \ldots, Y_{N}\right)$ $\leqq 0$ can be treated by the FOSM methods in the manner described earlier.

Implied in the preceding discussion is the fact that, for practical reliability analyses, it is often convenient to work with random variable representations of the load effects rather than random process representations. Indeed, the existing load combination rules to be mentioned in Sec. 4.8.6 capitalize on this fact.

Finding the distribution function $\mathrm{F}_{Q_{T}}(\alpha)$ of $Q_{T}$ defined in Eq. 4.107 is equivalent either to the problem of evaluating the probability $P\left(Q_{T}>\alpha\right)$ that the random process $Y(t)$ will exceed a specified (barrier) level $\alpha(>0)$ in a given time interval $[0, T]$ or to the problem of deriving the distribution function $F_{T_{\alpha}}(t)$ of the time (instant) $T_{\alpha}$ at which the process crosses the (barrier) level for the first time. The former problem is referred to 
- as the level crossing problem while the latter as the first passage time problem. Since the event that $Q_{T}$ is smaller than $\alpha$ is identical with the event that $T_{\alpha}$ is larger than $T$, one can show that

$$
F_{Q_{T}}(\alpha)=1-F_{T_{\alpha}}(T)
$$

Unfortunately, no exact solution is currently available for these problems except for certain simple cașes.

\subsubsection{Bounding Techniques and Other Approximations}

Shinozuka [1964] and Shinozuka and Yao [1967] were among the first to study the level crossing problem in the context of structural safety and reliability, and developed a bounding technique in order to provide an approximate solution to the problem. Actually, their technique can apply to the level crossing problem with time-dependent two-sided barriers. The twosided barrier problem is concerned with the excursion probability $P_{Y}\left(T_{;}-\alpha_{1}\right.$, $\left.\alpha_{2}\right)$ that $Y(t)$ will be found outside the two barriers at $-\alpha_{1}\left(\alpha_{1}>0\right)$ and $\alpha_{2}$ $(>0)$ in the time interval $[0, T]$. The equivalence similar to that in Eq. 4.110 between the level crossing probability and the first passage probability exists also for the case of two-sided barrier problems. When $\alpha_{1} \rightarrow \infty$, the two-sided barrier problem reduces to a standard (one-sided) level crossing problem. The barriers $-\alpha_{1}$ and $\alpha_{2}$ can be (deterministic) functions of time; $\alpha_{1}=\alpha_{1}(t)>0$ and $\alpha_{2}=\alpha_{2}(t)>0$. This bounding technique reiterated in a recent report by Shinozuka [July 1980], indicates the following lower and upper bounds for the probability $P_{Y}\left(T ;-\alpha_{1}, \alpha_{2}\right)$ :

$$
\begin{aligned}
P\left\{A_{1}\left(t_{1}\right) \cup A_{2}\left(t_{2}\right)\right\}<P_{Y}\left(T ;-\alpha_{1}, \alpha_{2}\right)<\int_{0}^{T}\left[v^{+}\left(\alpha_{2} ; t\right)+v^{-}\left(-\alpha_{1} ; t\right)\right] d t \\
+P\left\{X(0) \leqq-\alpha_{1}(0)\right\}+P\left\{X(0) \geqq \alpha_{2}(0)\right\}-P\left\{A_{1}\left(t_{1}\right) \cap A_{2}\left(t_{2}\right)\right\}
\end{aligned}
$$


where $A_{1}\left(t_{1}\right)$ represents the event that, at any time instant $t_{1}\left(0 \leqq t_{1} \leq T\right)$, $Y\left(t_{1}\right)$ is less than or equal to $-\alpha_{1}\left(t_{1}\right)$. Symbolically, this is written as

$$
A_{1}\left(t_{1}\right)=\left\{x\left(t_{1}\right) \leqq-\alpha_{1}\left(t_{1}\right) ; \quad 0 \leqq t_{1} \leqq T\right\}
$$

Similarly,

$$
A_{2}\left(t_{2}\right)=\left\{x\left(t_{2}\right) \geqq \alpha_{2}\left(t_{2}\right) ; \quad 0 \leqq t_{1} \leq T\right\}
$$

The quantity $\nu^{+}\left(\alpha_{2} ; t\right)$ in Eq. 4.111 is the expected rate of upcrossing of the barrier $\alpha_{2}(t)$ by $Y(t)$ at time $t$ and is given by

$$
v^{+}\left(\alpha_{2} ; t\right)=\int_{\alpha_{2}}^{\infty}\left(\dot{\gamma}-\dot{\alpha}_{2}\right) f\left(\alpha_{2}, \dot{\gamma} ; t\right) d \dot{\gamma}
$$

while the quantity $v^{-}\left(-\alpha_{1} ; t\right)$ is the expected rate of downcrossing of the barrier $-\alpha_{1}(t)$ by $Y(t)$ at time $t$ and is equal to

$$
\nu^{-}\left(-\alpha_{1} ; t\right)=\int_{-\infty}^{-\dot{\alpha}_{1}}\left(-\dot{\alpha}_{1}-\dot{\gamma}\right) f\left(-\alpha_{1}, \dot{\gamma} ; t\right) d \dot{\gamma}
$$

where $f(Y, \dot{Y} ; t)$ is the joint density function of $Y(t)$ and $\dot{Y}(t)$. The time dependency of this joint density function disappears if $Y(t)$ and therefore $\dot{Y}(t)$ are stationary.

As emphasized by Shinozuka himself $[1964,1966]$ and later recognized by Lin [1967], this bounding technique is especially suited for evaluating the excursion probability under the condition that the process $Y(t)$ is relatively intense only within a short duration of time. Indeed, the numerical examples presented by Shinozuka [1964, 1966] and Shinozuka et al [1968] dealing with single- and multiple-degree-of-freedom systems, respectively, subjected to nonstationary Gaussian earthquake-like random processes, verify that the bounds evaluated on the basis of Eq. 4.111 are reasonably close 
under the conditions just mentioned. This observation will prove to be important when the above bounding technique is applied to the load combination problem involving intermittent load effects as shown in Fig. $4.8(\mathrm{~b})$ and (c). In many civil engineering applications, the barriers $-\alpha_{1}$ and $\alpha_{2}$ are constant at least in approximation and one of these barriers is much larger (in the absolute value sense) than the other; $\alpha_{1} \gg \alpha_{2}$ or $\alpha_{1} \ll \alpha_{2}$. Therefore, the one-sided level crossing problem with $\alpha_{1}=\infty$ will mainly be considered below. Under the further assumption that $Y(t)$ is stationary, the upper and lower bounds for the excursion probability $P_{Y}(T ;-\infty, \alpha)$ can then be obtained as

$$
A_{02}<P_{Y}(T ;-\infty, \alpha)<v(\alpha) T+A_{20}
$$

where

$$
A_{20}=P\{Y(0) \geq \alpha\}
$$

and $\alpha$ is written for $\alpha_{2}$ for simplicity,

$$
v(\alpha)=\int_{0}^{\infty}\left(\dot{y}-\alpha_{2}\right) f\left(\alpha_{2}, \dot{\gamma}\right) d \dot{y}
$$

In Eq. 4.118, the joint density function of $Y(t)$ and $\dot{Y}(t)$ is time-invariant because of the assumed stationarity of $Y(t)$.

The bounding technique developed above, however, is immediately useful only when the joint density function $f(Y, \dot{Y} ; t)$ of $Y(t)$ and $\dot{Y}(t)$ can be found, since it is needed to evaluate the up-and down-crossing rate in Eqs. 4.114 and 4.115 , respectively. If all the component load effects $Y_{i}(t)$ are Gaussian processes, then $Y(t)$ and $\dot{Y}(t)$ are also jointly Gaussian. The prospect of analytically deriving such a joint density function for $Y(t)$ consisting of the arbitrary component processes $Y_{i}(t)$ appears dim, however. 
The bounding technique is now extended and applied to the cases involving $N$ statistically independent, and possibly intermittent load effects under the following assumptions: (1) One is dealing with a one-sided barrier problem as defined above. (2) Each load effect, $Y_{\mathbf{j}}(t)$, is stationary while it is acting or, to be more precise, one occurrence of $Y_{i}(t)$ initiated at time $t$ and terminated at time $t+t_{0}$, can be modeled in terms of a stationary random process multiplied by a rectangular pulse of unit height between $t$ and $t+t_{0}$. Under these assumptions, the following crossing rates can be evaluated:

$v_{i}(\alpha)=v_{i}=$ constant rate of up-crossing by the load response $i, v_{i}(t)$, when $Y_{j}(t)$ is the only load effect that is on.

$v_{i+j}(\alpha)=v_{i+j}=$ constant rate of up-crossing by the sum $Y_{i+j}(t)$ of the load responses $Y_{j}(t)$ and $Y_{j}(t)(i \neq j)$ when $Y_{j}(t)$ and $Y_{j}(t)$ are the only load effects that are on simultaneously.

and similar definitions for $v_{i+j+k}$, etc. For the evaluation of these upcrossings, Eq. 4.118 can be used if the density function $f(Y, \dot{Y})$ is replaced by $f\left(Y_{i}, \dot{Y}_{j}\right), f\left(Y_{i+j}, \dot{Y}_{i+j}\right)$, etc. Using these crossing rates, one can obtain an upper bound for the probability of structural failure due to the first excursion above the level $\alpha$ in the time interval $[0, T]$ which is given by

$$
\begin{gathered}
T \bar{v}(\alpha)=T \sum_{j=1}^{N} P_{1}^{(i)} v_{i}+\sum_{i=1}^{N} \sum_{\substack{j \neq j \\
j \neq 1}}^{N} p_{2}^{(i, j)} v_{i+j}+\sum_{\substack{i=1 \\
i \neq j}}^{N} \sum_{\substack{j=1 \\
k \neq i}}^{N} \sum_{\substack{j \neq k \\
k=1}}^{N} p_{3}^{(i, j, k)} v_{i+j+k} \\
\left.+\ldots+P_{N} v_{1+2+\ldots+N}\right\}
\end{gathered}
$$

where $\bar{v}(\alpha)$, representing the sum in the braces above, is the overall average 
up-crossing rate and $P_{1}^{(i)}, p_{2}^{(i, j)}, p_{3}^{(i, j, k)}, \ldots, P_{N}$ are defined as follows. $P_{0}=$ the probability that none of the load effects are on.

$P_{1}^{(i)}=$ the probability that load effect $Y_{i}(t)$ and only load effect $Y_{i}(t)$ is on.

$p_{2}^{(i, j)}=$ the probability that only the load effects $Y_{i}(t)$ and $Y_{j}(t)$ are simultaneously on.

$P_{3}^{(i, j, k)}=$ the probability that only the load effects $Y_{i}(t), Y_{j}(t)$ and $Y_{k}(t)$ are simultaneously on.

$P_{N}=$ the probability that all the load effects are simultaneously on.

The statement that $\bar{T}(\alpha)$ is an upper bound for the probability of structural failure is valid provided, of course, that the resisting strength $R$ in the limit state equation, Eq. 4.109, is deterministic and equal to $\alpha$. If $R$ is not deterministic, then the upper bound should be modified to

$$
P_{f} \leqq T \int_{-\infty}^{\infty} \bar{v}(\alpha) f_{R}(\alpha) d \alpha
$$

where $f_{R}(\cdot)=$ the density function of $R$. Often, the distribution function $F_{Q_{T}}(\alpha)$ of the lifetime maximum $Q=Q_{T}$ (Eq. 4.107) is approximated by

$$
F_{Q}(\alpha)=\exp \{-\bar{v}(\alpha) T\}
$$

or the first passage time probability $F_{T_{\alpha}}(T)$, Eq. 4.110, by 


$$
F_{T_{\alpha}}(T)=1-\exp \{-\bar{v}(\alpha) T\}
$$

Since Eq. 4.4 can also be written as

$$
P_{f}=P(R \leqq Q)=\int_{-\infty}^{\infty}\left[1-F_{Q}(\alpha)\right] f_{R}(\alpha) d \alpha
$$

the use of Eq. 4.122 in Eq. 4.124 results in an approximation of the probability of structural failure when the resisting strength $R$ is random. This approximation is widely used, primarily for its relative ease of mathematical tractability. It is usually believed that the probability of structural failure $P_{f}$ obtained from Eq. 4.124 with $F_{Q}(\alpha)$ approximated by Eq. 4.122 produces a conservative result. Some observations are made in this respect in Appendix $G$.

A similar result to that of Eq. 4.119 was reported by Winterstein [1980]. The probabilities in Eq. 4.119 depend on the distribution functions of the interarrival times and durations of the load effects. Using the queue theory, Shinozuka [July 1980; Appendix H] systematically derived the analytical expressions for these probabilities under the assumptions: (a) The load effects are statistically independent; (b) the interarrival times for individual load effects are exponentially distributed (i.e., imply Poisson arrivals); and (c) the duration of each occurrence of each load effect is also exponentially distributed, with, however, the restriction that it cannot last beyond the arrival time of the next occurrence of the same load effect:

$$
\begin{aligned}
& P_{0}=1 / \prod_{m=1}^{N}\left(1+\rho_{m}\right) \\
& P_{1}^{(i)}=\rho_{i} / \prod_{m=1}^{N}\left(1+\rho_{m}\right)
\end{aligned}
$$




$$
\begin{gathered}
p_{2}^{(i, j)}=\rho_{i} \rho_{j} / \prod_{m=1}^{N}\left(1+\rho_{m}\right) \\
P_{3}^{(i, j, k)}=\rho_{i=1} \rho_{j} \rho_{k} / \prod_{m=1}^{N}\left(1+\rho_{m}\right) \\
\vdots \\
P_{N}=\prod_{n=1}^{N} \rho_{n} / \underset{m=1}{N}\left(1+\rho_{m}\right)
\end{gathered}
$$

where

$$
\rho_{i}=\lambda_{j} / \mu_{i} \quad(i=1,2, \ldots, N)
$$

with $\lambda_{i}=$ the arrival rate of load effect $i$ and $\mu_{i}=$ the rate at which the duration of one occurrence of the load effect $i$ terminates. The ratios $\rho_{i}$ are equivalent to the so-called traffic intensity in the queue theory and they are much smaller than unity for the transient load effects (Fig. 4.8(c)) which occur rarely and last for short periods of time; e.g., earthquake and wind load effects.

Shinozuka and Tan [August 1980; Appendix I] extended the work by Shinozuka [JuTy 1980] so that the duration of each occurrence of an individual load effect distributes in accordance not only with the exponential distribution but also with the Erlang distribution. However, the restriction used in the earlier work that the duration of each occurrence of an individual load effect cannot last beyond the arrival time of the next occurrence of the same load effect, was retained in the analysis. If the duration of load effect $i$ is distributed as an Erlang distribution, it can be interpreted as the sum of $k$ independent random variables, each of which is distributed exponentially with a density function $\mu_{i} \exp \left(-\mu_{i} t\right)$. The density 
function of the Erlang distribution then takes the following analytical form:

$$
g(t)=\left(k_{i} / \mu_{i}\right)^{k_{i}} t^{k_{i}-1} e^{-k_{i} \mu_{i} t} /\left(k_{i}-1\right) ! \quad t \geq 0
$$

with parameters $k_{i}$ and $\mu_{i}$. Assuming aga in that the interarrival time of load effect $i$ is distributed according to the exponential distribution with the density function $\lambda_{i} \exp \left(-\lambda_{i} t\right)$, the probabilities defined in Eq. 4.120 can be shown to be:

$P_{0}=1 / \Delta$

$p_{1}^{(i)}=\sum_{n_{i}=1}^{k_{i}} \rho_{i}\left(1+\rho_{i}\right)^{n_{i}-1} / \Delta$

$p_{2}^{(i, j)}=\sum_{n_{i}=1}^{k_{i}} \sum_{n_{j}=1}^{k_{j}} \rho_{i}\left(1+\rho_{i}\right)^{n_{i}-1} \rho_{j}\left(1+\rho_{j}\right)^{n_{j}-1} / \Delta$

$p_{3}^{(i, j, k)}=\sum_{n_{j}=1}^{k_{i}} \sum_{n_{j}=1}^{k_{j}} \sum_{n_{k}=1}^{k_{k}} \rho_{i}\left(1+\rho_{j}\right)^{n_{i}-1} \rho_{j}\left(1+\rho_{j}\right)^{n_{j}-1} \rho_{k}\left(1+\rho_{k}\right)^{n_{k}-1} / \Delta$

$P_{N}=\sum_{n_{1}=1}^{k_{1}} \sum_{n_{2}=1}^{k_{2}} \cdots \sum_{n_{N}=1}^{k_{N}} \rho_{1}\left(1+\rho_{1}\right)^{n_{1}-1} \rho_{2}\left(1+\rho_{2}\right)^{n_{2}-1} \ldots \rho_{N}\left(1+\rho_{N}\right)^{n_{N}-1} / \Delta$

where

$$
\Delta=\prod_{j=1}^{N}\left(1+\rho_{j}\right)^{k}
$$

With respect to the up-crossing rates $v_{i+j}$, etc., the following approximation was derived by Larrabee and Cornell [1978] for the independent processes $Y_{i}(t)$ and $Y_{j}(t)$ : 


$$
v_{i+j}(\alpha)=\int_{-\infty}^{\infty} f_{i}(y) \nu_{j}(\alpha-y) d y+\int_{-\infty}^{\infty} f_{j}(y) \nu_{i}(\alpha-y) d y
$$

where $f_{i}(\alpha)=$ the density function of $Y_{j}(t)$ and $v_{j}(\alpha)=$ the up-crossing rate of the barrier $\alpha$ by $Y_{i}(t)$. The validity of Eq. 4.130a does not require that $Y_{i}(t)$ and $Y_{j}(t)$ be stationary. Eq. 4.130a can also be written symbolically as

$$
v_{i+j} \simeq v_{i} * f_{j}+v_{j} * f_{j}
$$

where the asterisk indicates the appropriate convolution integral. The extension of Eqs. 4.130a and $b$ to those cases where more than two independent load effects must be considered, is straightforward: For example,

$$
\begin{gathered}
v_{1+2+3}=v_{1} * f_{2} * f_{3}+v_{2} * f_{1} * f_{3}+v_{3} * f_{2} * f_{1} \\
\vdots \\
v_{1+2+\ldots+N} \approx v_{1} * \\
f_{2} * f_{3} * \ldots * f_{N}+v_{2} * f_{1} * f_{3} * \ldots * f_{N}+ \\
\ldots+v_{N} * f_{1} * f_{2} * \ldots * f_{N-1}
\end{gathered}
$$

Since, for example, the Fourier transform of the convolution $v_{1} * f_{2} * f_{3}$ * $\ldots * f_{N}$ can be written as the product $\bar{v}_{1} \cdot \bar{f}_{2} \cdot \bar{f}_{3} \cdot \ldots \cdot \bar{f}_{N}$ in which the super bar indicates the Fourier transform of the corresponding function, the convolutions on the right-hand sides of Eqs. 130b, $c$ and $d$ can easily be performed by means of FFT (Fast Fourier Transform) techniques particularly for those cases where the closed form solutions cannot be obtained or the component functions $v$ 's and f's are given only numerically: First, take the Fourier transform of the $v^{\prime} s$ and $f^{\prime} s$. Then, form the product, say $\bar{v}_{1} \cdot \bar{f}_{2} \cdot$ $\ldots . \cdot \bar{f}_{N}$ and take the inverse fourier transform of the product. 
The degree of approximation to be achieved by making use of Eqs. 4.130 for the evaluation of the up-crossing rate depends on the stochastic nature of the component processes. It appears that the approximation tends to produce conservative results. For example, the statistically independent processes $Y_{1}(t)$ and $Y_{2}(t)$ are both stationary and Gaussian with mean zero, the up-crossing rate $v_{1+2}(\alpha)$ of $Y(t)=Y_{1}(t)+Y_{2}(t)$ can be easily evaluated as

$$
v_{1+2}(\alpha)=\frac{1}{2 \pi} \sqrt{\frac{\dot{\sigma}_{1}^{2}+\dot{\sigma}_{2}^{2}}{\sigma_{1}^{2}+\sigma_{2}^{2}}} \exp \left\{-\frac{1}{2} \alpha^{2} /\left(\sigma_{1}^{2}+\sigma_{2}^{2}\right)\right\}
$$

while Eq. 4.130a (or b) results in

$$
v_{1+2}(\alpha)=\frac{1}{2 \pi} \frac{\dot{\sigma}_{1}+\dot{\sigma}_{2}}{\sqrt{\sigma_{1}^{2}+\sigma_{2}^{2}}} \exp \left\{-\frac{1}{2} \alpha^{2} /\left(\sigma_{1}^{2}+\sigma_{2}^{2}\right)\right\}
$$

where $\sigma_{i}$ and $\dot{\sigma}_{i}$ are the standard deviations of $Y_{i}(t)$ and $Y_{j}(t)$, respectively, $(i=1,2)$. The approximate up-crossing rate indicated by Eq. 4.132 is clearly larger than the exact value given by Eq. 4.131. In Appendix G, another example and further comments with respect to the nature of this approximation for the up-crossing rate are presented.

The fact that relatively straightforward results as exemplified by Eqs. $4.125,4.128$ and 4.130 have been obtained in the present analysis, is primarily due to the assumed statistical independence among the component processes $Y(t)$. A theory that can account for the possible statistical dependence among the $Y(t)$ 's has not been fully developed at this time, however. Also, the assumption that the combined load effect is given by a linear com- 
bination of $Y(t)$ 's (Eq. 4.108), together with the simple limit state equation given by Eq. 4.109, gives rise to the relatively simple and analytically tractable approximations for the probability of structural failure as given by Eqs. 4.121, 4.122 and 4.124. Indeed, if the nonlinear combinations of load effects are to be considered for their total effect, it appears that one can only deal with highly specialized component processes and limit state equations [Veneziano et al., 1977 and Breitung and Rackwitz, 1979].

\subsubsection{Load-Coincidence Method}

An al ternative method was proposed by Wen $[1977,1980$ a $]$ based on a consideration of the load coincidence. The survival of the structure (1 imit state not being reached in the structure's lifetime) requires survival under individual loadings as well as the coincidence of two or more loadings. Therefore, for independent loadings, the structural reliability is given in approximation by

$1-P_{f} \simeq \exp \left[-\sum_{i=1}^{N} v_{i} p_{i}^{m} t-\sum_{i=1}^{N} \sum_{j=1}^{N} v_{i j} p_{i j}^{m} t-\sum_{i=1}^{N} \sum_{j=1}^{N} \sum_{k=1}^{N} v_{i j k} p_{i j k}^{m} t-\ldots ..\right]$

where, following Wen's notation, $t$ is the lifetime of the structure, and $\lambda_{i}$ and $\mu_{d_{i}}$ are the arrival rate and mean duration (of each occurrence) of the $i$-th load effect $Y_{i}(t)$. Then

$v_{i}=\lambda_{i}, v_{i j}=\lambda_{i} \lambda_{j}\left(\mu_{d_{i}}+\mu_{d_{j}}\right), v_{i j k}=\lambda_{i} \lambda \lambda_{j} \lambda_{k}\left(\mu_{d_{i}} \mu_{d_{j}}+\mu_{d_{j}} \mu_{d_{k}}+\mu_{d_{i}} \mu_{d_{k}}\right)$

are the mean rate of occurrence of $Y_{j}(t)$ only, the coincidence of $Y_{i}(t)$ and 
$Y_{j}(t)$ only and the coincidence of $Y_{i}(t), Y_{j}(t)$ and $Y_{k}(t)$ only, etc. The $p_{i}^{m}, p_{i j}^{m}, p_{i j k}^{m}$, etc. are the conditional probabilities of failure (m-th limit state being reached) given the occurrence and coincidence of loads, respectively. Wen asserted that his formulation (Eq. 4.133) was generally conservative and could easily handle the reliability problem of linear or nonlinear, static or dynamic structural systems, and further verified that its accuracy was within the practical range by extensive Monte Carlo simulations [Wen; 1977, 1980a].

If the total load effect can be given by the sum of the component effects and if the limit state condition can be specified in terms of a onesided barrier problem with $\alpha_{2}=\alpha=$ constant, the conditional probabilities $p_{i}^{m}, p_{i j}^{m}$, etc. can be evaluated, respectively, as the probabilities that $Y_{i}(t)$ $Y_{i}(t)+Y_{j}(t)$, etc. will exceed the level $\alpha$. In fact, in this case, Eq. 4.133 is identical to Eq. 4.122 if $v_{i} T, v_{i+j} T$, etc. in Eq. 4.119 represent the true probabilities of the level crossings rather than their corresponding upper bounds. The conditional probabilities, however, must be determined through a possibly more intricate structural reliability analysis if the total load effect is a nonlinear function of the component load effects and/or the limit state equations are more complicated.

Primarily with the aid of the pulse process model in which the occurrence time is modeled by a point process while the duration and intensity given occurrence by random variables, the load-coincidence method represented by Eq. 4.133 was extended by Wen [1980b, 1981] so that one can deal with the possible intra- and inter-process dependencies. In fact, the extension permits one to treat those pulse processes in which the occurrence times, load intensities and durations may be correlated within each process 
and/or between processes. Intra-process (within-load effect) dependencies can arise between the intensity and duration. For example, storms with longer durations tend to produce more intense wind loads. Equally important is the occurrence dependence (clustering) which is observed, for example, when after-shocks follow the main ground shock due to an earthquake, and when a large number of tornadoes are spawned by a single storm. Also, it is possible that a correlation exists between the intensities of individual pulses. Examples of inter-process (between-load effects) dependencies are also numerous: a common storm can induce dependent wind, wave and surge loads on off- or near-shore structures, and a severe earthquake load effect is more likely to accompany intense LOCA loadings due to possible pipebreaks caused by such an earthquake. Such dependencies are taken into consideration in the load effect combination analysis introducing multi-variate clustering point process models for the dependent occurrences, imbedded Gauss-Markov sequence models for intensity dependencies, and multi-variate distribution models for intensity-duration correlations. Approximate analytical results similar to that given in Eq. 4.133 were obtained in closed form and verified by Monte Carlo simulations [Wen, 1980b and 1981] considering two or three component processes. It was then found that, compared with the results for independent loadings, (1) the durationintensity correlation within the same load effect causes a slight increase in the exceedance probability for a lifetime combined maximum at high threshold levels, (2) the intensity dependence and occurrence clustering within the same load effect cause a moderate decrease in such a probability at lower threshold values and have little effect at high threshold values, (3) the load effect coincidence rate is extremely sensitive to occurrence 
clustering between the load effects and the rate can increase by several orders of magnitude (depending on the characteristics of the clustering dependence which then produces a much higher probability of exceedance at high threshold values, and (4) the intensity correlation between the load effects is important at high threshold levels. As mentioned earlier, however, further study is needed to fully understand the effect of these dependencies on the probability of structural failure.

\subsubsection{Method of Laplace Transform and Renewal Equation}

An alternative way of evaluating the up-crossing rate is through the use of the Laplace transform. Breitung and Rackwitz [1979] obtained the crossing rates in the Laplace transform doma in for the summation of Poisson square waves and for filtered Poisson processes with rectangular pulses. Such a procedure gives the exact solution for the crossing rates, with, however, the disadvantage that numerical transformations are required first for finding the Laplace transform of the probability density functions of the load intensities (closed form solutions exist as an exception rather than as a rule) and later in the inversion to find the crossing rate in the time domain.

Gaver and Jacobs [1979] took advantage of the renewal property of the Poisson square wave and formulated the exact solution of the probability distribution of the maximum value when such a process is combined with independent shock loadings represented by Poisson spikes (with random intensity). Assume that the spikes will not occur simultaneously with the changes in the Poisson square wave. Furthermore, let $\lambda_{1}$ and $\lambda_{2}$ be the mean change rate for the Poisson square wave and the mean occurrence rate for the spikes, respectively, and $x_{1}$ and $x_{2}$ be the corresponding intensi- 
ties. The renewal equation for the probability distribution of the combined maximum $x_{m}=\max \left\{x_{1}(\tau)+x_{2}(\tau), 0<\tau \leq t\right\}$ is

$$
\begin{aligned}
F_{X_{m}}(r, t) & =e^{-\lambda_{2} t} \int_{0}^{r} \exp \left[-\lambda_{2} t G_{X_{2}}(r-s)\right] f_{X_{1}}(s) d s \\
& +\int_{0}^{t} \lambda_{1} e^{-\lambda 1^{\tau}} \int_{0}^{r} \exp \left[-\lambda_{2} \tau G_{X_{2}}(r-s)\right] F_{X_{m}}(r, t-\tau) f_{X_{1}}(s) d s d \tau
\end{aligned}
$$

in which $G_{X_{i}}(\cdot)=1-F_{X_{i}}(\cdot)$. Eq. 4.135 is easily solved in the Laplace transform domain. Similarly to the Rackwitz method, numerical procedures are required in finding both the transform and the inversion. Some closed form mean first excursion times and asymptotic solutions of $F_{X_{m}}(r, t)$ as $t \rightarrow x$ are obtained.

Extension of Eq. 4.135 to the case where part of the Poisson shocks occur simultaneously with the changes in the Poisson square wave was investigated by George [1980], and Winterstein [1980]. No numerical examples or comparisons with approximate solutions are given. The renewal equation-Laplace transform method represents a fresh approach to the load combination problem. It is attractive since one can obtain the exact solution provided that there are no difficulties in the numerical transform and inversion. However, extending this method to the combination of other types of load effects (e.g., intermittent load effects, load effects with clustered occurrences) appears rather difficult, since these types of load effects do not usually possess the renewal property from which the simplicity of this method originates. 


\subsubsection{Errors of Some Load Combination Rules}

In a reliability-based design, the structure should be proportioned so that a specified reliability is built into it. For this purpose, the foregoing method of analysis may be used to arrive at such a design through an iterative process. However, explicit reliability-based load combination rules that designers can follow for routine checks are not yet ayailable. It is therefore of practical importance to examine the accuracies of load combination rules currently in use. Strictly speaking, such rules are for design use and not for analysis; one can only check whether a specific structure designed according to such rules indeed has the intended safety leve1. However, in most design codes such safety levels are not indicated, at least not explicitly (e.g., in probability terms). Almost all such rules in essence simplify the problem of the combination of random processes to that of random variables and as a result, inaccuracy is introduced. In view of this, wen [1980 a,b] examined the validity of existing rules by comparing the probability distribution function for the combined effect derived analytically with those of the combined effect evaluated in accordance with the rules. In doing so, however, the sum of two independent Poisson renewal processes with rectangular or triangular pulses was considered, since such processes have been studied extensively and for which analytical results have already been verified.

\section{Load Reduction Factor Method (LFR)}

Many building codes recommend a reduction factor (or allowing an increase in the allowable stress) when transient load effects are combined. For the combination of two load effects, the reduction factor method states that the design combined load effect is equal to the maximum among the in- 
dividual design load effects and their sum multiplied by a reduction factor $C$. Therefore, it impl ies the following approximation when a lifetime maximum combined load effect is considered.

$\operatorname{Max}\left[Y_{1}(t)+Y_{2}(t)\right] \simeq \operatorname{Max}\left\{\operatorname{Max}\left[Y_{1}(t)\right], \operatorname{Max}\left[Y_{2}(t)\right], C\left(\operatorname{Max}\left[Y_{1}(t)\right]+\right.\right.$

$\left.\left.\operatorname{Max}\left[Y_{2}(t)\right]\right)\right\}$

Commonly used values of $C$ range from 0.66 for combinations of three loads to $0.7,0.75$ or 0.8 for combinations of two loads.

\section{Square-Root-of-Sum-of-Squares Rule (SSRS)}

The square-root-of-sum-of-squares law commonly used in structural dynamics has been applied to load combination problems in the following manner.

$$
\operatorname{Max}\left[Y_{1}(t)+Y_{2}(t)\right] \simeq \sqrt{\operatorname{Max}\left[Y_{1}(t)\right]^{2}+\operatorname{Max}\left[Y_{2}(t)\right]^{2}}
$$

\section{Companion Action Factor Method (CAF)}

In this method, when combining two design load effects, one takes the sum of one of the design load effects and the other design load effect multiplied by a factor, therefore implying, for a lifetime maximum load effects,

$\operatorname{Max}\left[Y_{1}(t)+Y_{2}(t)\right]=\operatorname{Max}\left\{\operatorname{Max}\left[Y_{1}(t)\right]+C_{2} \operatorname{Max}\left[Y_{2}(t)\right], \quad C_{1} \operatorname{Max}\left[Y_{1}(t)\right]+\right.$

$$
\left.\operatorname{Max}\left[Y_{2}(t)\right]\right\}
$$

where $c_{1}$ and $c_{2}$ are the companion action factors, ranging from 0.2 to 0.8 depending on the type of load under consideration and safety level desired. 
Turkstra's Rule (TR)

When combining load effects, one can take the sum of the maximum of one load effect and the instantaneous (arbitrary-point-in-time) value of the other.

$\operatorname{Max}\left[Y_{1}(t)+Y_{2}(t)\right] \simeq \operatorname{Max}\left[\operatorname{Max}\left[Y_{7}(t)\right]+Y_{2}, \operatorname{Max}\left[Y_{2}(t)\right]+Y_{1}\right\}$

in which $Y_{1}$ and $Y_{2}$ are the arbitrary-point-in-time values. Since the above procedure neglects the possibility that the combined maximum can occur at times other than when one of the processes reaches a maximum, it is always unconservative [Bosshard 1975]. This procedure has been used in a recently proposed probabilistic design code for steel construction [Ravindra and Galambos 1977] and also by Ellingwood et al [1980] in its general form for dealing with the load combination problems that involve, for example, dead, live and wind loads:

$$
\operatorname{Max} Y(t) \simeq \operatorname{Max}_{i}\left[\max _{\substack{0 \leq t \leq T \\ \underline{1}}} Y_{j}(t)+\sum_{\substack{j=1 \\ j \neq i}}^{N} Y_{j}(t)\right]
$$

where $Y(t)$ is given by Eq. 4.108 .

\section{Comments}

The general conclusion is that LRF, SRSS and CAF methods are adequate in combining transient load effects at a relatively low (mean or median of the lifetime maximum) level. Overly unconservative designs may result when combining much higher design load effects. On the other hand, TR works best in combining load effects when at least one is always present but could lead to quite unconservative designs when combining transient load effects at the low to intermediate levels. Also, if LRF and CAF are to be used, the proper choices of C's must be made depending on the reliability levels to be speci- 
fied and load types to be considered.

\subsection{Conclusion and Summary}

Recent developments in structural reliability analys is fundamentals have been reviewed. New interpretations for these theoretical developments are offered and alternative methods are proposed to evaluate some of those quantities crucial to structural reliability estimation.

(1) The advanced first order second moment methods are interpreted as procedures for locating the point of common tangency between the limit state surface $g_{j}(\underline{x})=0$ and the quadratic surface $\underline{x}^{\top} \underline{\Lambda}^{-1} \underline{x}=\gamma_{0}^{2}$ where $\underline{x}$ represents a standardized random vector with its components having the covariance matrix 1. If such a point of common tangency, referred to as the design point, is found at $\underline{x}=\underline{x}_{\star}$ for a particular value of $\gamma_{0}^{2}$, the safety index $\beta$ associated with the linear expansion $g_{0}(\underline{x})$ of $g_{j}(\underline{x})$ at that point can be shown to be $\pm \sqrt{\gamma_{0}^{2}}$ with the aid of the Lagrange multiplier methods (under certain assumptions on the curvature of the limit state surface). The sign in front of the square root is chosen so that $\beta>0$ if $\Delta g_{1}=\underline{x}_{*} \underline{G}_{*}<0$ and $\beta<0$ if $\Delta g_{1}>0$ where $\underline{G}_{\star}$ is the component vector of the gradient of $g_{j}(\underline{x})$ evaluated at the design point.

(2) The (absolute value of) the safety index can be interpreted as the distance between the design point and the origin of the $\underline{x}$ space if $\underline{x}$ is Gaussian with uncorrelated components. If they are correlated, it represents the distance between the design point and origin in the $y$ space where $\underline{y}$ is also standardized Gaussian and obtained through a linear transformation of $\underline{x}$ so that the components of $\underline{y}$ are uncorrelated.

(3) The design point $\underline{x}_{*}$ is the point of maximum likelihood if $\underline{x}$ is Gaussian, whether or not its components are uncorrelated.

(4) The value $\Phi(-\beta)$ of the standardized Gaussian distribution at $-\beta$ yields a lower bound of the limit state probability $P_{f}$ under certain conditions imposed 
on the curvature of the limit state surface. Similarly, an upper bound for $P_{f}$ can be established using the $x^{2}$-distribution with $n$ degrees of freedom where $n$ is the dimension of the random vector $\underline{x}$. If $\underline{x}$ is not Gaussian, it can be transformed into $\underline{u}$, which is Gaussian with independent components, without much difficulty as long as the components of $\underline{x}$ are independent of each other. These bounds often result in an interval for $P_{f}$ which is too wide to be of practical value. More importantly, these bounds are not valid if a number of limit state conditions must be considered. It is suggested, therefore, that the Monte Carlo method be used to evaluate the usual integral expression for $P_{f}$ when direct integrations are not practical, numerically or otherwise. In this respect, it is also shown that the area and volume integrals for $P_{f}$ dealing with, respectively, two- and three-dimensional problems, reduce to contour and surface integrals along the limit state surface. Efforts towards the extension of this approach to multidimensional cases are suggested. Once $P_{f}$ is estimated, the safety index may be defined as $\beta=-\Phi\left(P_{f}\right)$.

(5) Based on an interpretation of the advanced first order second moment method as described in Item 1 above, the exact method of evaluating the safety index and locating the design point has been articulated. Furthermore, as a possibly more practical alternative to the exact solution method, as well as to the Rackwitz iterative procedure, it is proposed that the point of maximum likelihood be used as the design point $\underline{x}_{*}$ even when $\underline{x}$ is not Gaussian. This design point can be transformed into $\underline{u}_{*}$ through the same transformation that transforms $\underline{x}$ into the (standardized) Gaussian vector $\underline{u}$ with independent compontents. (This transformation is made under the assumption that the components of $\underline{x}$ are independent of each other. Otherwise, such a transformation will probably cause considerable analytical difficulties, if not total intractability). The safety index is then computed as $\beta= \pm\left(\underline{u}_{*}^{\top} \underline{u}_{*}\right)^{\frac{1}{2}}$. The design point $\underline{u}_{*}$ in the 
- u space and the safety index $B$ thus evaluated are approximations to the exact $\underline{u}_{*}$ and the corresponding safety index, since the exact $\underline{u}_{*}$ should be obtained as the point of maximum likelihood in the $\underline{u}$ space. However, these approximations are expected to be close to the exact values. Indeed, a numerical example performed did produce approximations that are extremely close to the exact values. This alternative approach proposed by Shinozuka [September 1980] is considered to be included in the advanced first order second moment (FOSM) methods whenever a reference is made to them.

(6) The procedures described above determine, in one way or another, the design point $x_{i}^{\star}, x_{2}^{\star}, \ldots, x_{n}^{\star}$ in the original variable space. Such a design point satisfies the limit state condition $g\left(x_{1}^{*}, x_{2}^{\star}, \ldots, x_{n}^{*}\right)=0$ and is associated with a safety index (or a probability of failure). By expressing $X_{i}^{*}$ in terms of their corresponding nominal values $X_{N_{i}}$, one obtains $X_{i}^{*}=\gamma_{i} X_{N_{i}}$ where the factors $\gamma_{i}$ are defined as the load or resistance factors. Since the design point depends on the (postulated) limit state and the safety index (or the probability of failure) while the nominal values $X_{N_{i}}$ are usually fixed, the load and resistance factors depend on the limit state to be postulated as well as on the target safety index (or target probability of failure).

(7) In dealing with a structure subjected to multiple time-varying loads, the following suggestions are made to be consistent with the current state of the art of structural reliability theory: (a) load effects $Y_{j}(t)$, rather than loads $x_{j}(t)$, be used to construct the limit state equation, (b) load effects $Y_{j}(t)$ be assumed to be linearly related to loads $X_{j}(t),(c)$ total load effect $Y(t)$ be the sum of the component load effects $Y_{j}(t) ; Y(t)$ $=\sum_{i=1}^{N} Y_{i}(t)$, (d) the limit state equation be of 1 inear form, $R-Q \leq 0$, with $R$ representing the structural resisting capacity while $Q=Q_{T}$, the maximum of $Y(t)$ during the lifetime $T$ of the structure. 
(8) The total load effect $Y(t)$ under consideration may consist of component effects which are time invariant except for one, say $Y_{N}(t)$. Then, $Y_{i}(t)$ may be considered as random variables $Y_{i}(i=1,2, \ldots, N-1)$. Generally, the distribution function of $Q_{N T}$ representing the maximum value of $Y_{N}(t)$ during the lifetime $T$ of the structure can be estimated empirically or analytically at least in approximation. Then, the lifetime maximum $Q=Q_{T}$ of $Y(t)$ can be written as $Q=Q_{N T}+\sum_{i=1}^{N-1} Y_{i}$, and the limit state equation becomes $R-Q_{N T}-\sum_{j=1}^{N} Y_{i} \leq 0$. Since this 1 imit state equation involves random variables only, the safety index $\beta$ can be computed with the aid of the FOSM methods, and the design point determined. The load and resistance factors can then be determined in the manner described in the recent report by Ellingwood et al [1980]. The probability of failure $P_{f}$ can also be estimated if the distribution (or density) functions of $R$ and $Q$ are known.

(9) Under assumptions (a) - (d) in (7) above, the 1 imit state equation $R-Q \leq 0$ can be interpreted as the failure condition associated with the first excursion problem with a one-sided barrier at $R=a>0$.

(10) Under the further assumption that the $Y(t)$ 's are all statistically independent and stationary while acting, the overall average upcrossing rate $\bar{v}(\alpha)$ is derived. If some of the $Y(t)$ 's are of the intermittent type, this rate involves $P_{1}^{(i)}=$ the probability that $Y_{i}(t)$ alone is on, $p_{2}^{(i, j)}=$ the probability that $Y_{i}(t)$ and $\gamma_{j}(t)$ alone are on, .... $P_{N}=$ the probability that all the $Y(t)$ 's are on. Exact analytical expressions for these probabilities are derived for those cases where the intermittent load effects occur in accordance with the Poisson law and the durations of these occurrences are governed by either the exponential distribu- 
tion function or the Erlang distribution function.

(11) The overall up-crossing rate $\bar{v}(\alpha)$ also involves $v_{i}=$ the rate of up-crossing of $\alpha$ by $Y_{i}(t), v_{i+j}=$ the rate of up-crossing of $\alpha$ by $Y_{i}(t)$ $+Y_{j}(t)$, etc. Approximate expressions for $v_{i+j}, v_{i+j+k}$, etc. can be given in terms of convolution integrals involving the $v$ 's and density function $f$ 's of the $Y(t)$ 's.

(12) The analytical expression for the overall up-crossing rate $\bar{v}(\alpha)$ is also valid for the case where all the $Y(t)$ 's are always acting. In this case, however, $P_{1}^{(i)}, P_{2}^{(i, j)}$, etc. must be set equal to zero except for $P_{N}$ which is then equal to unity.

(13) The overall average up-crossing rate $\bar{v}(\alpha)$ multiplied by the lifetime $T$ of the structure is an upper bound for the probability of structural failure $P_{f}$ if $R=\alpha$ is deterministic. Otherwise, the upper bound is modified to $T \int_{-\infty}^{\infty} \bar{v}(\alpha) f_{R}(\alpha) d \alpha$.

(14) The probability of failure $P_{f}$ under a deterministic $R=\alpha$ may be approximated by $1-\exp \{-T \bar{v}(\alpha)\}$. Then, if $R$ is random, $P_{f} \simeq \int_{-\infty}^{\infty}[1-$ $\exp \{-\bar{T}(\alpha)\}\} \quad f_{R}(\alpha) d \alpha$.

(15) The load-coincidence method developed by Wen is also reviewed. The method provides an alternative form for the probability of structural failure $P_{f}$. This alternative form is identical to the expression 1 $\exp \left\{-T_{\bar{v}}(\alpha)\right\}$ indicated above under certain conditions including assumptions (a) - (d) in (7). Also, it is more immediately applicable when the total load effect is a nonlinear combination of component load effects and when there are dependencies among the occurrence, intensity and duration within each load effect and/or between load effects. Wen's results indicate that the effect of these dependencies on the probability of structural failure 
may be quite significant. These results, while based on a combination study for two or three component load effects modelled as pulse processes, provide useful insight into these dependency questions and also serve as a foothold for future investigation.

(16) A Laplace transform method for evaluating the up-crossing rate for Poisson square waves and filtered Poisson processes is reviewed. While the theoretical attractiveness of the approach is obvious, it appears that further efforts are necessary to make the method capable of dealing with more realistic models of load effects.

(17) The validity of the load combination rules that are currently recommended and used is examined by Wen. They include the Load Reduction Factor Method, Square-Root-of-Sum-of-Squares Rule, Companion Action Factor Method and Turkstra's Rule. For the sum of two independent Poisson renewal processes with rectangular or triangular pulses, it was found that these rules sometimes produce overly unconservative resulsts.

(18) The determination of load and resistance factors on the basis of the FOSM methods requires that the total load effect be expressed in terms of a sum of the random variables representing the component load effects. Unfortunately, this requirement cannot be rigorously met if two or more component load effects must be idealized as random processes rather than as random variables. In this case, however, Turkstra's rule can be invoked in approximation so that the FOSM methods can be applied for the determination of the load and resistance factors. In this regard, it is mentioned that the comments made in (10) - (16) above pertain to the evaluation of the probability of failure but not to the determination of the load and resistance factors by means of the FOSM methods. 
(19) The observation made in (18) above indicates the necessity for developing a method more directly based on the probability of failure, by which the load and resistance factors can be determined when two or more component load effects are randomly time-varying. 
Table 4.1 Location of Design Point and Safety Index Values

\begin{tabular}{|l|c|c|c|c|c|l|}
\hline & $x^{\star}$ & $x^{\star}(\mathrm{ksi})$ & $z^{\star}$ & $Z^{\star}\left(\mathrm{in}^{3}\right)$ & $u^{\star}$ & $B$ \\
\hline Iteration & -3.629 & 24.21 & -2.552 & 47.11 & $(-4.469)$ & 5.144 \\
\hline Approximate & -3.639 & 24.17 & -2.530 & 47.17 & $(-4.489)$ & $5.153^{+}$ \\
\hline Exact & $(-3.625)$ & 24.22 & -2.568 & 47.07 & -4.465 & $5.151^{+}$ \\
\hline
\end{tabular}

( ) indicates a quantity calculated either from $x^{*}$ or from $u^{*}$

$+\quad$ indicates that $B$ is obtained as $\sqrt{\left(u^{*}\right)^{2}+\left(z^{*}\right)^{2}}$

$X^{*}$ and $Z^{*}$ indicate the design point in the original $X-Z$ space

Table 4.2 Design Points, Limit State Probabilities and Domains of Integration

\begin{tabular}{|c|c|c|c|c|c|}
\hline$T$ & & & & \multicolumn{2}{|c|}{$A$} \\
\cline { 4 - 6 } & $x_{1}^{*}$ & $x_{2}^{*}$ & $p_{f}$ & $x_{1}$ & $x_{2}$ \\
\hline 50 & -2.20 & -1.72 & $2.32(2.32) \times 10^{-3}$ & $-9.67 \sim 4.33$ & $-6.80 \sim 5.2$ \\
\hline 20 & -3.92 & -3.38 & $2.0(2.07) \times 10^{-7}$ & $-9.67 \sim 2.33$ & $-6.80 \sim 1.10$ \\
\hline 5 & -0.32 & -6.48 & $5.5(5.47) \times 10^{-11}$ & $-9.67 \sim 10.33$ & $-6.80 \sim-0.60$ \\
\hline
\end{tabular}

+ Fiessler, et al.

+ Monte Carlo simulation (sample size $=1,000$ ) 

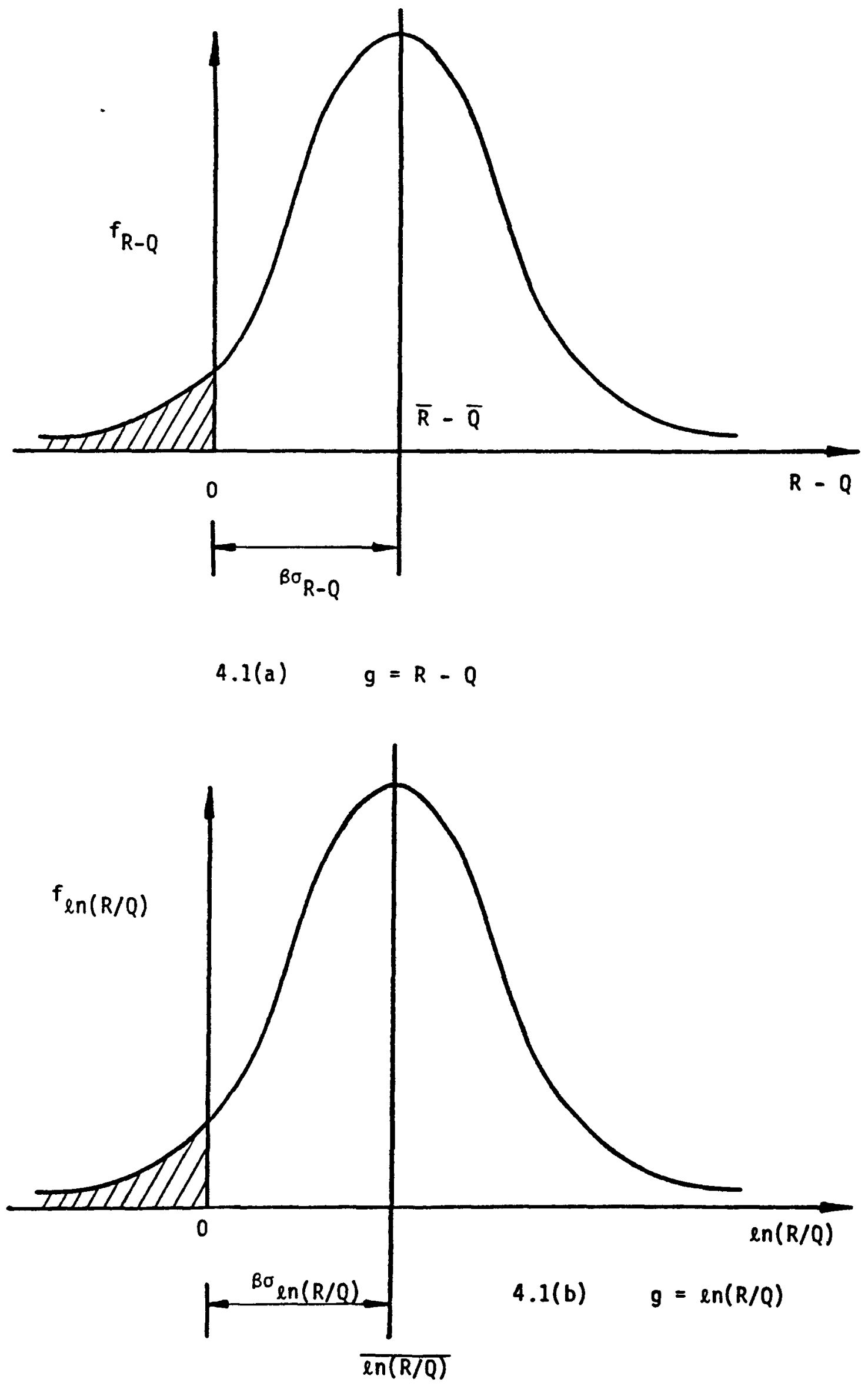

Fig. 4.1 Illustration of the Reliability Index Concept $4-67$ 


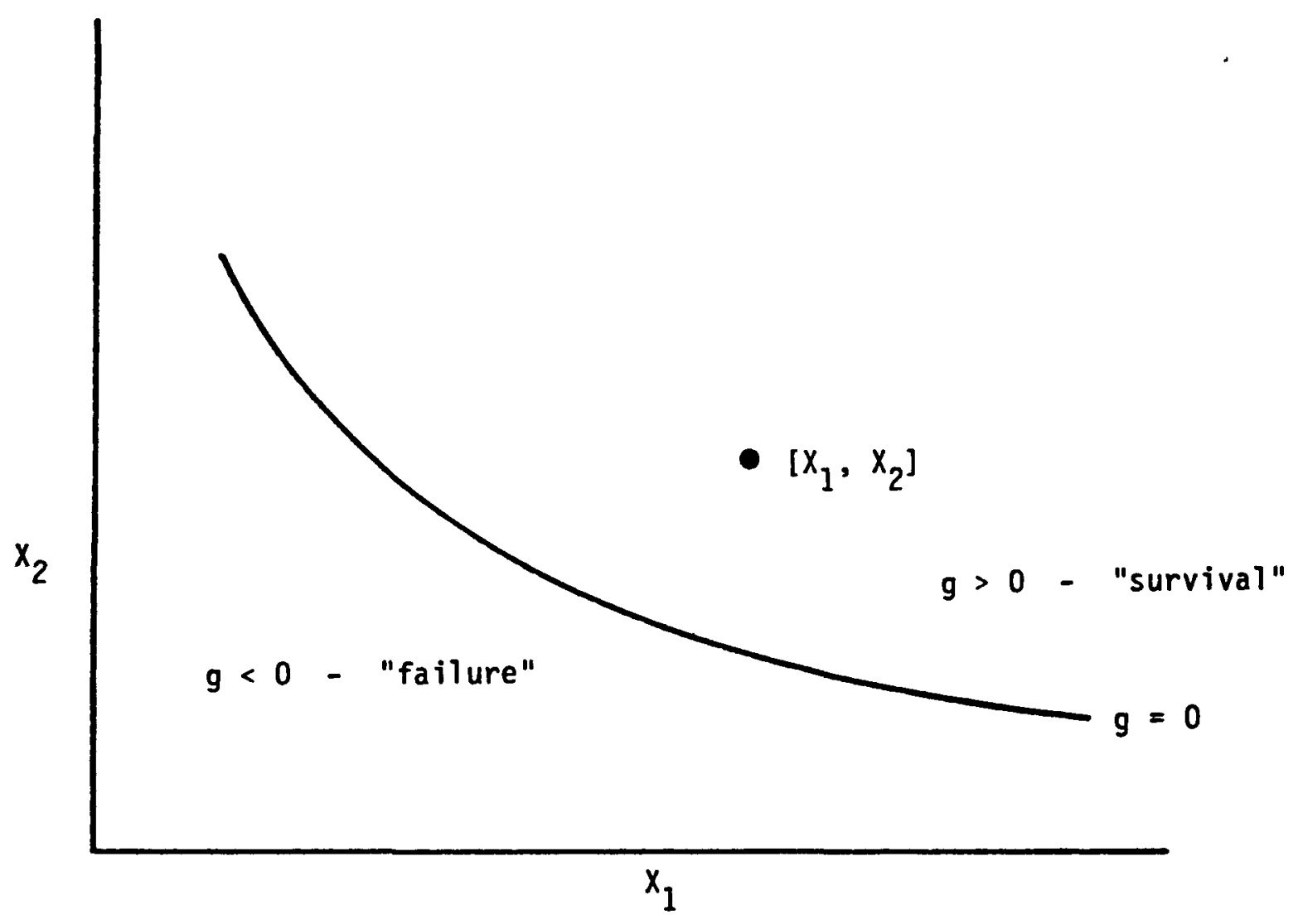

\section{$4.2(a)$}

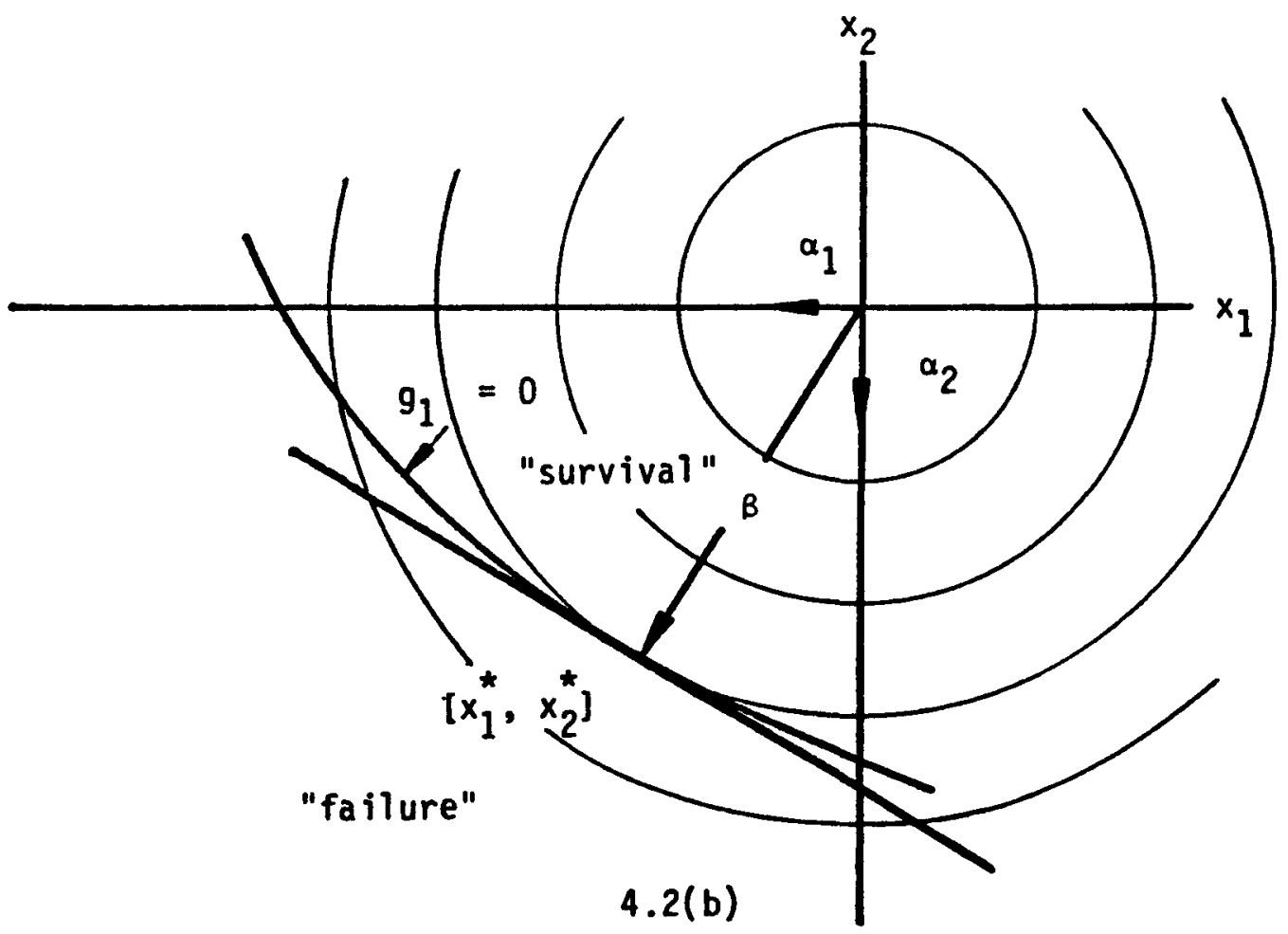

Fig. 4.2 Formulation of Safety Analysis in Original and Standardized Variable Coordinate 


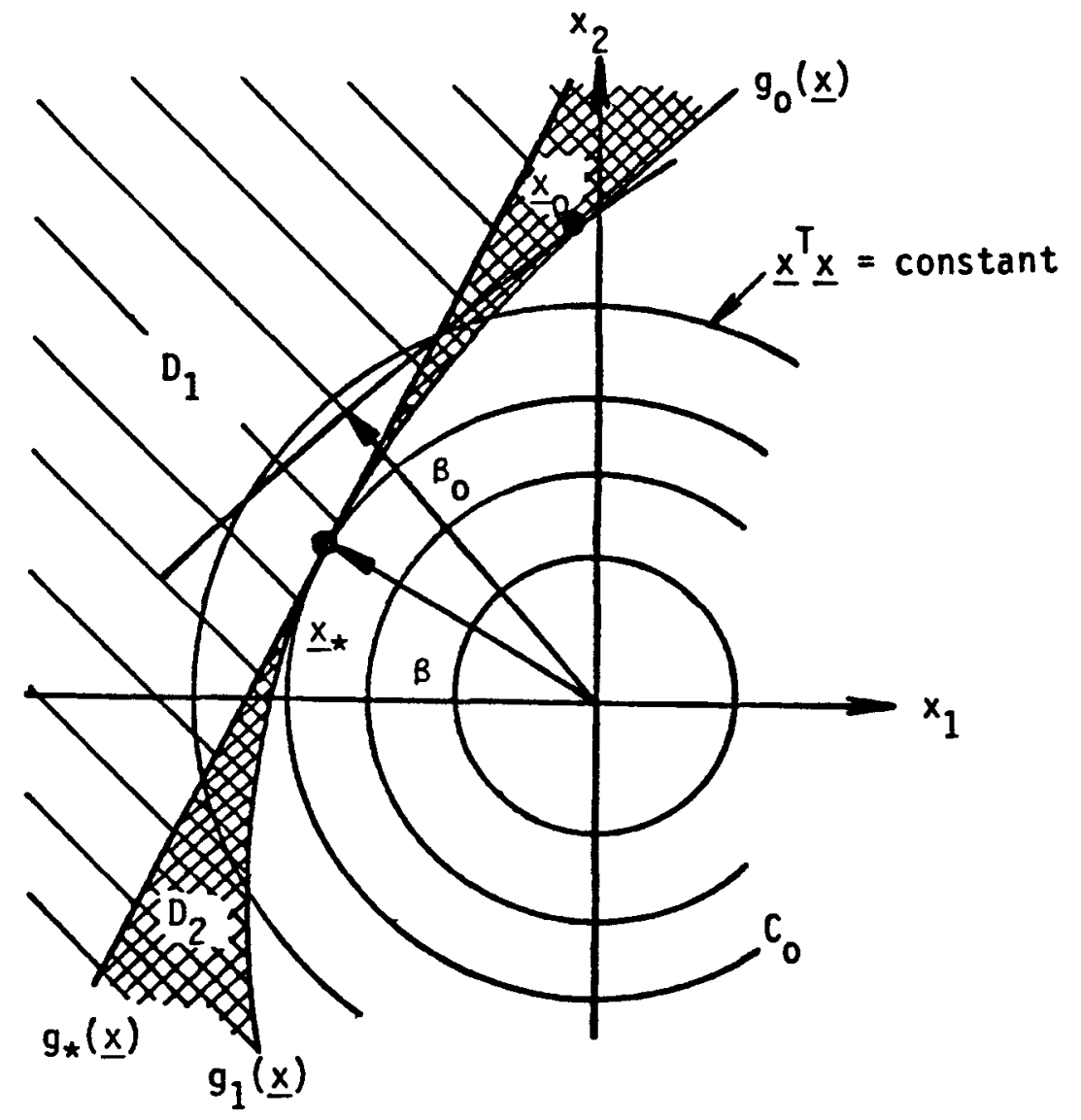

Fig. 4.3 Determination of Design Point When Components of $\underline{x}$ Are Uncorrelated 


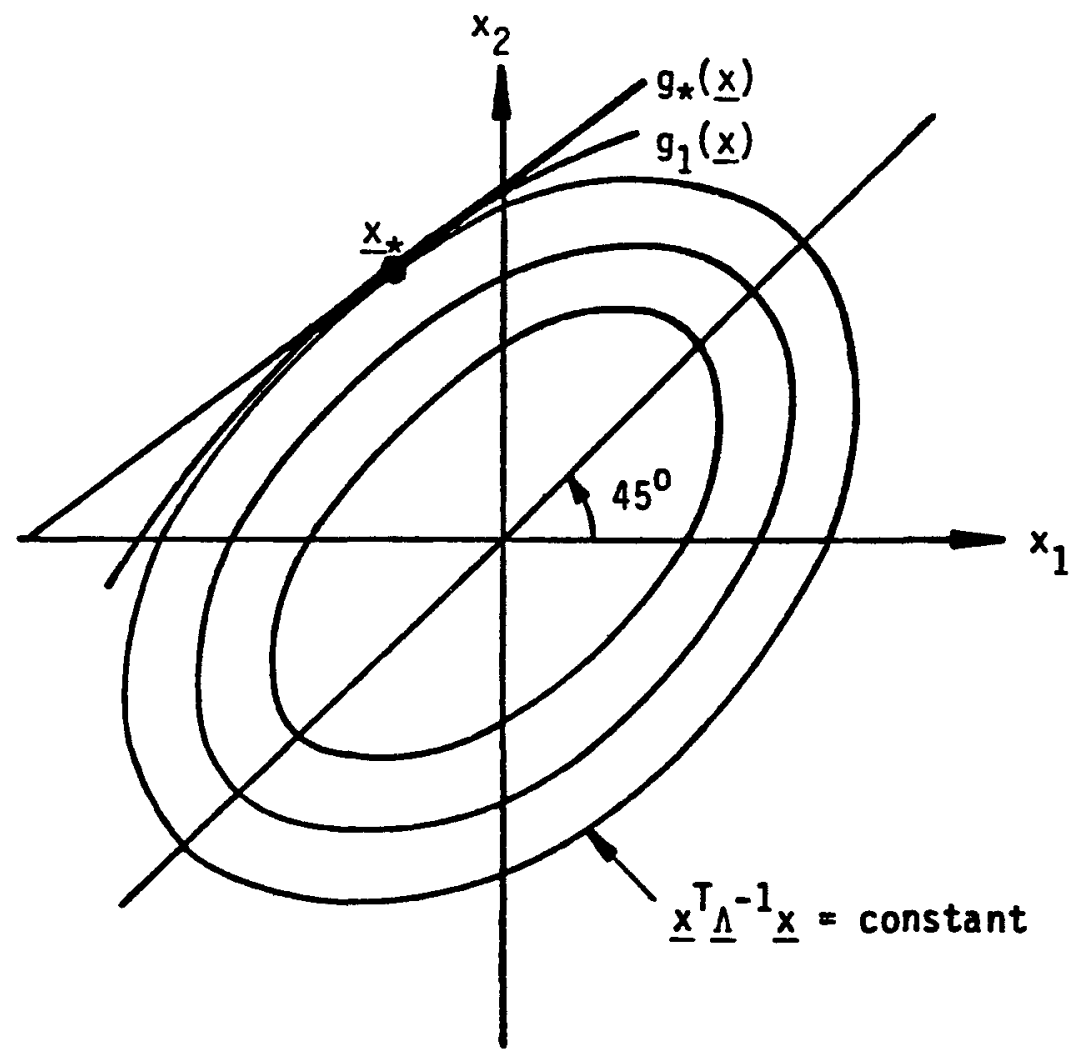

Fig. 4.4 Determination of Design Point When Components of $\underline{x}$ Are Correlated 


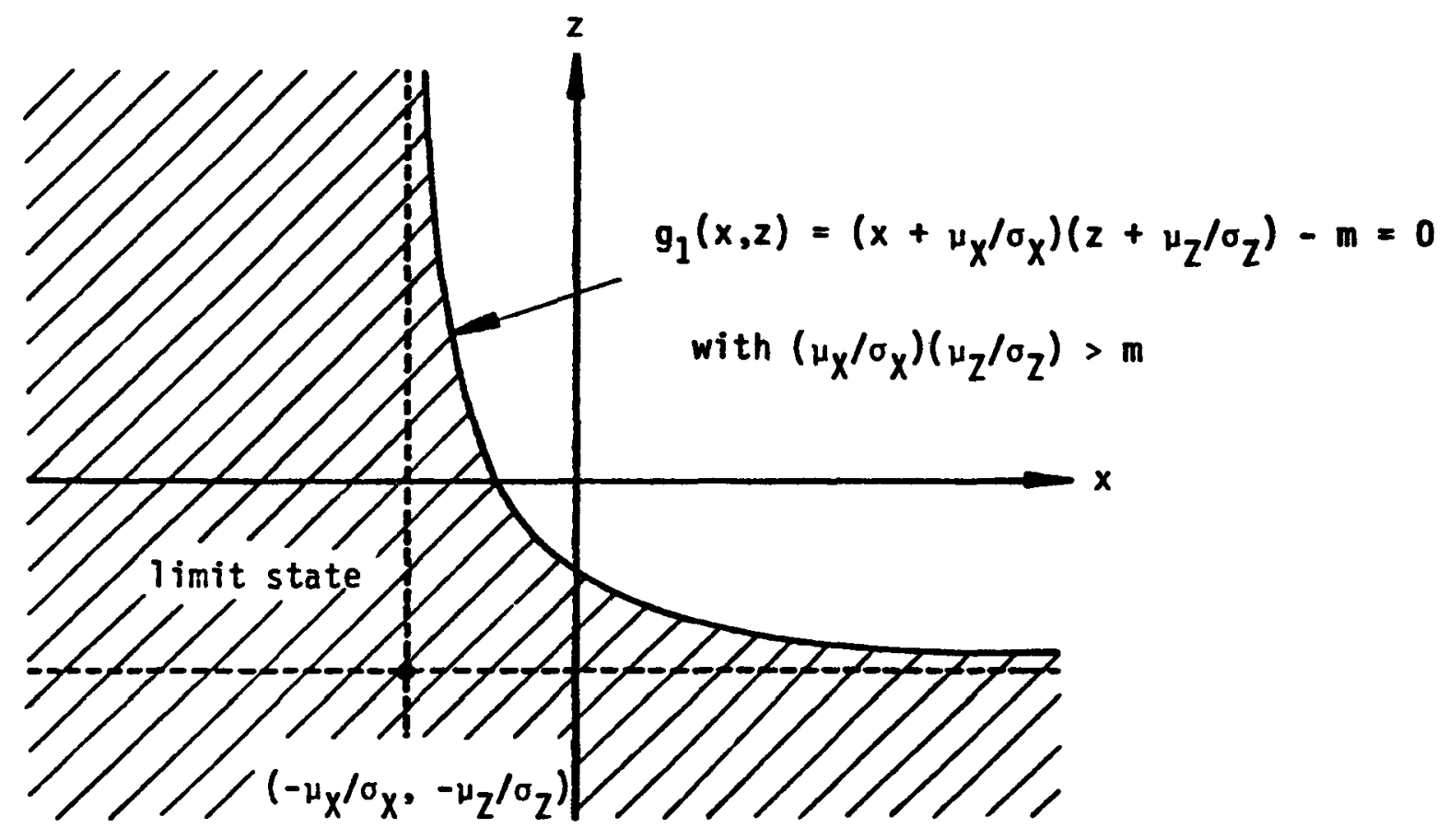

Fig. 4.5 Limit State Surface in the $x-z$ Space 


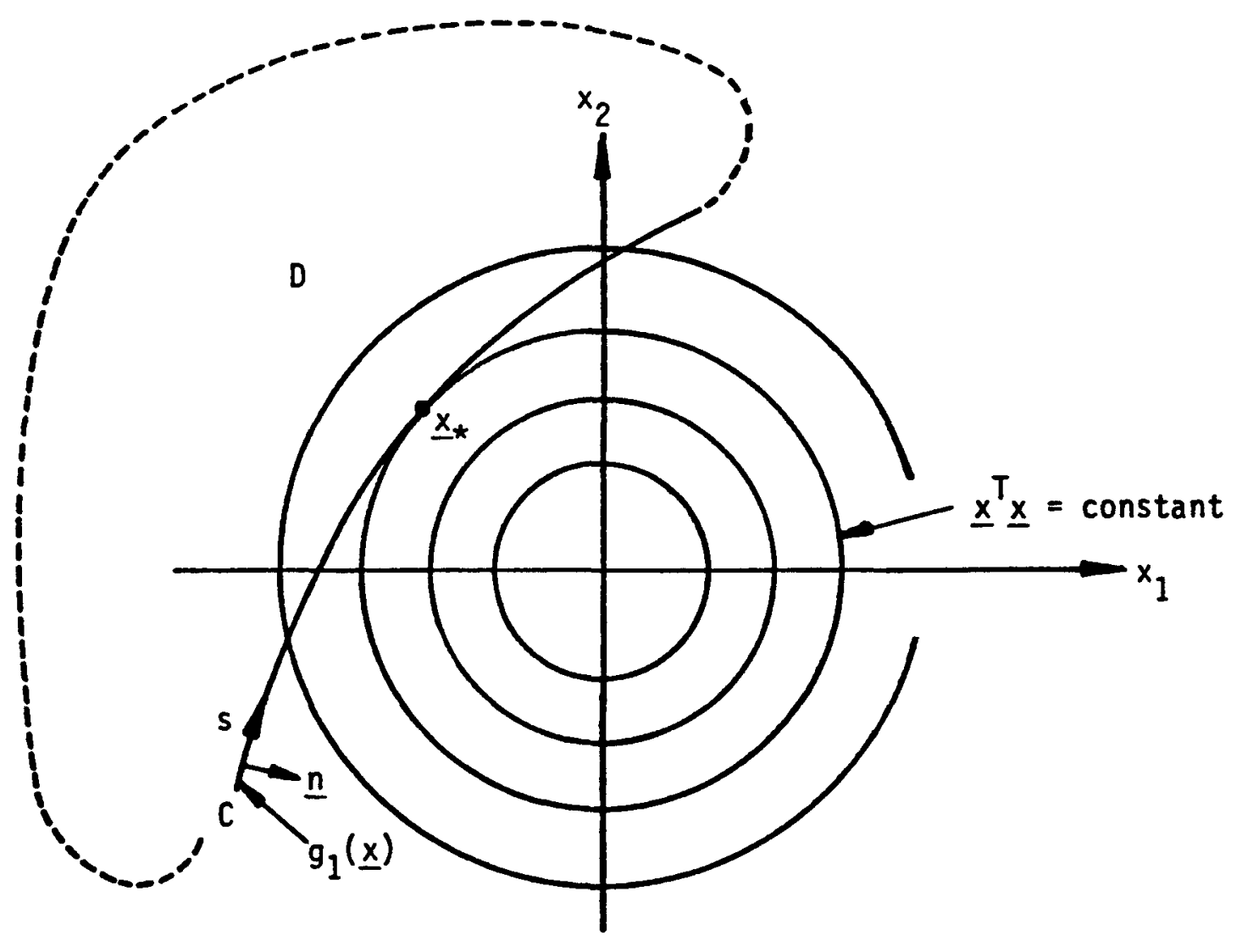

Fig. 4.6 Use of Contour Integral for the Estimation of the Limit State Probabilities 


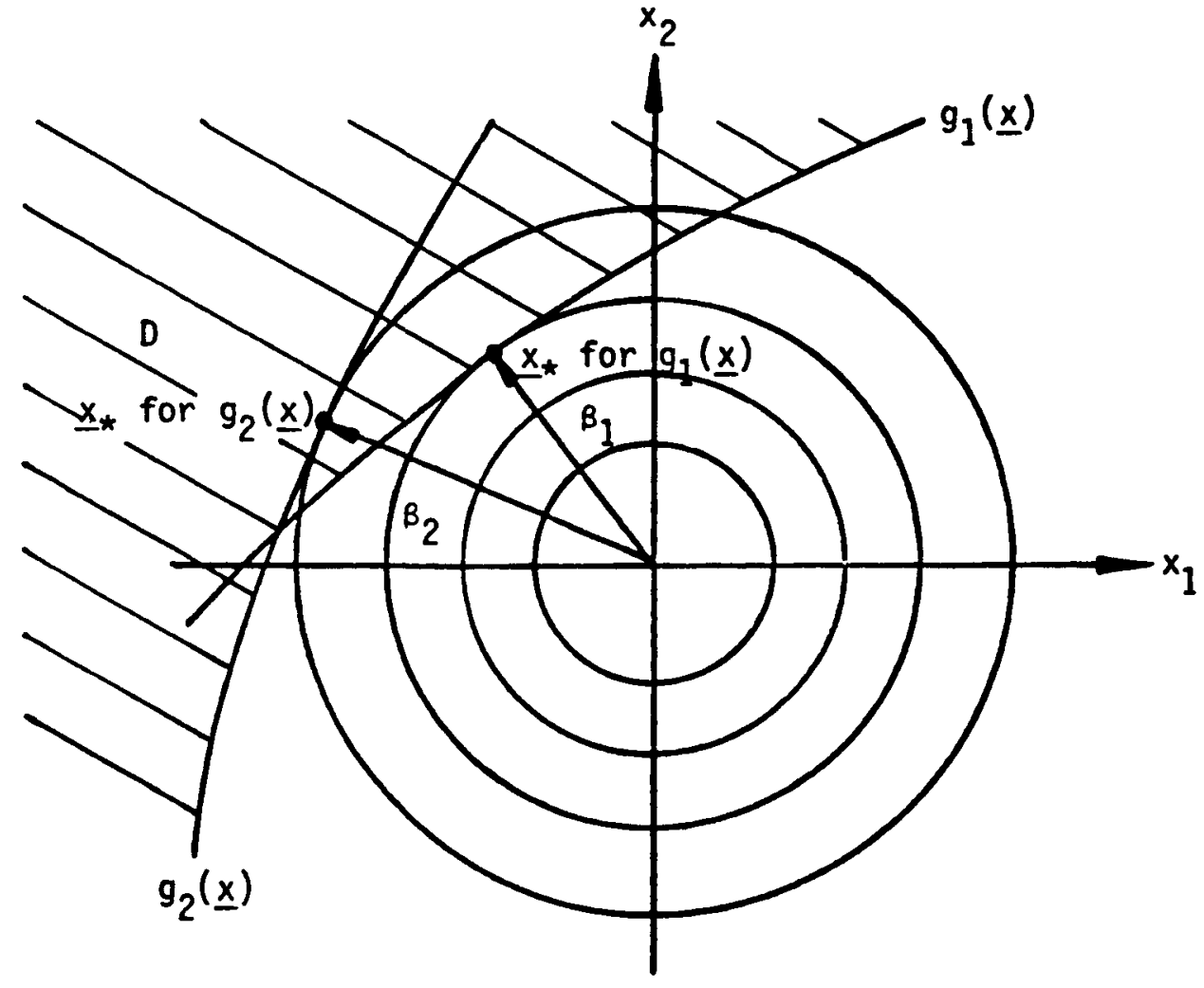

Fig. 4.7 Limit State Under Multiple Limit Conditions 


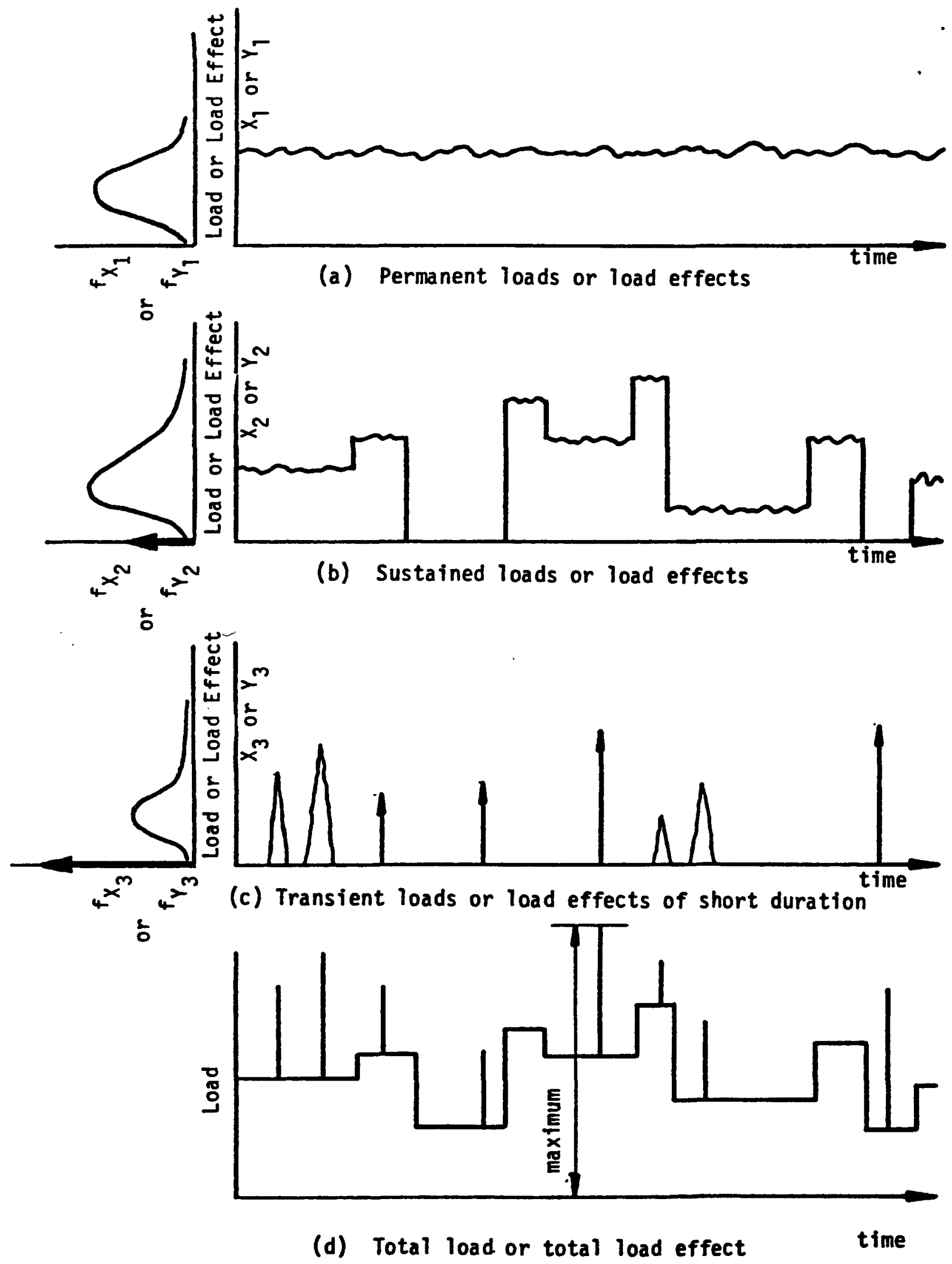

Fig. 4.8 Schematic Representation of Various Loads and Load Effects 


\section{REFERENCES}

1) Abbey, R. F., Jr., Risk Probabilities Associated with Tornado Windspeeds, Proceedings of the Symposium on Tornadoes Assessment of Knowledge and Implications for Man, Lubbock, TX, June 22-24, 1976, pp. 177-236.

2) Abbey, R. F., Fujita, T. T., Use of Tornado Path Lengths and Gradations of Damage to Assess Tornado Intensity Probabilities, Preprint, Ninth Conf. on Severe Local Storms, Norman, OK, Oct. 21-23, 1975, pp. 286-348. Published by American Meteorological Society, Boston, MA.

3) Algermissen, S. T., Perkins, D. M., A Probabilistic Estimate of Maximum Acceleration in Rock in the Contiguous United States, U. S. Geological Survey Open File Report 76-416, 1976.

4) An Approach to Quantitative Safety Goals for Nuclear Plants, Advisory Committee on Reactor Safeguards, NRC, NUREG-0739, October 1980.

5) Ang, A., Corne11, C. A., Reliability Bases of Structural Safety and Design, Journal of the Structural Division, ASCE, Vol. 100, No. ST9, Proc. Paper 10777, September 1975, pp. 1755-1769.

6) ASME Boiler and Pressure Vessel Code, Section III, Division 1, ASME, New York.

7) ASME Boiler and Pressure Vessel Code Section III, Division 2 (ACI-35980), ASME, New York.

8) Batts, M., et al., Estimation of Hurricane Wind Speeds in the United States, Building Science Series No. 118, National Bureau of Standards, Washington, D.C., 1979.

9) Bosshard, W., Report No. 20, J. A. Blume Earthquake Engineering Center, Stanford University, June 1975. 
10) Breitung, K. and Rackwitz, R., Nonlinear Combination of Poisson

Renewal Load Processes, Res. Rep. Technical Univeristy of Munich, Munich,. West Germany, March 1979.

11) Breitung, K. and Rackwitz, R., Upcrossing Rates for Rectangular Pulse Load Processes, SFB 96, Res. Rep. Technical University of Munich, Munich, West Germany, JuTy 1979.

12) Bresler, B. (Ed.), Reinforced Concrete Engineering, John Wiley \& Sons, New York, 1974, pp. 416-418.

13) Building Code Requirements for Minimum Design Loads in Buildings and Other Structures, (ANS A58.1, 1972), American National Standards Institute, New York, 1972.

14) Building Code Requirements for Reinforced Concrete, (ACI 318-77), American Concrete Institute, Detroit, MI, 1977.

15) Code Requirements for Nuclear Safety Related Concrete Structures

(ACI 349) American Concrete Institute, Detroit, MI, 1976.

16) Cornel1, C. A., Bounds on the Reliability of Structural Systems, Journal of the Structural Division, ASCE, Vo1. 93, No. ST1, Proc. Paper 5096, February 1968, pp. 171-200.

17) Corne11, C. A., A Probability Based Structural Code, Journal of the American Concrete Institute, Vo1. 66, No. 12, December 1969, pp. 974-985. 18) Ellingwood, B. R., Reliability Bases of Load and Resistance Factors. for Reinforced Concrete Design, Building Science Series No. 110, National Bureau of Standards, Washington, DC, February 1978, 95 pp.

19) Ellingwood, B., Galambos, T. V., MacGregor, J. G., and Cornel1, C. A., Development of a Probability Based Load Criterion for American National Standard A58, National Bureau of Standards Special Publication No. 577, June 1980, $222 \mathrm{pp}$.

20) Federal Regulation 10CRF100 Reactor Site Criteria, Appendix A Seismic and Geologic Siting Criteria for Nuclear Power Plants, November 1973. 
21) Fiessler, B., Neumann, H.-J. and Rackwitz, R., Quadratic Limit States in Structural Reliability, EMD Journal, ASCE, Vo1. 105, No. EM4, August 1979, pp. 661-676.

22) Freudenthal, A. M. and Shinozuka, M., Structural Safety under Conditions of U1timate Load Failure and Fatigue, WADD Technical Report 61-677, October 1961, pp. 15-19.

23) Freudentha1, A. M., Garre1ts, J., Shinozuka, M., The Analysis of Structural Safety, Journal of the Structural Division, ASCE, Vol. 92, No. ST1, Proc. Paper 4682, February 1966, pp. 267-325.

24) Galambos, T., Ravindra, M., Load and Resistance Factor Design, Journal of the Structural Division, ASCE, Vol.104, No. ST9, Proc. Paper 14008, September 1978, pp. 1335-1356.

25) Galambos, T. V., Ravindra, M., Properties of Steel for Use in LRFD, Journal of the Structural Division, ASCE, Vol. 104, No. ST9, Proc. Paper 14009, September 1978, pp. 1459-1468.

26) Garson, R., et al., Tornado Design Winds Based on Risk, Journal of the Structural Division, ASCE, Vol. 101, No. ST9, September 1975, pp. 1883-1896.

27) George, L., Peak Combined Load from a Bivariate Poisson Shock and Square Wave Process, ORSA/TIMS National Meetings, Colorado Springs, Co., Nov. $11-13,1980$.

28) Hal1, W. J., Mohraz, B., Newmark, N. H., Statistical Studies of Vertical and Horizontal Earthquake Spectra, N. M. Newmark Consulting Engineering Services, Urbana, IL, Jan. 1976, issued as NUREG-0003-1976. 29) Howard, G. E. et a1., Seismic Design of Nuclear Power Plants - An Assessment, Nuclear Engineering and Design, Vol. 38, 1976, pp. 385-461. 
30) Jacobs, P. A. and Gaver, D. P., Random Load Combination, TIMS/ORSA National Meetings, New Orleans, May 1979, to appear in S.I.A.M., Journal of Applied Math.

31) Larrabee, R. D. and Corne11, C. A., Approximate Stochastic Analysis of Combined Loading, Res. Rep. CER 78-28, M.I.T., September 1978.

32) Larabee, R., Corne11, C. A., A Combination Procedure for a Wide Class of Loading Processes, Probabilistic Mechanics and Structural Reliability, ASCE, January 1979.

33) Larrabee, R. D. and Cornell, C. A., Upcrossing Rate Solution for Load Combinations, J. Struct. Div. Proc. ASCE, 105 (ST1), January 1979, pp. 125-132.

34) Lin, Y. K., Probabilistic Theory of Structural Dynamics, McGraw-Hill, New York, 1967, pp. 322-332.

35) Lind, N. C., Optimal Reliability Analysis by Fast Convolution, EMD Journal, ASCE, Vol. 105, No. EM3, June 1979, pp, 447-452.

36) Markee, E. H., Beckerley, J. G., and Sanders, K. E., Technical Basis for Interim Regional Tornado Criteria, WASH-1300, U.S. Atomic Energy Commission, May 1974.

37) Mark II Containment Dynamic Forcing Functions Information Report, NEDO-21061, General Electric Co. and Sargent and Lundy Engineers, September 1975.

38) McGuire, R., Seismic Structural Response Risk Analysis, Incorporating Peak Response Regressions on Earthquake Magnitude and Distance, Dept. of Civil Engineering Report No. 399, M.I.T., August 1974. 39) Meyer, C., Design Methodology for Nuclear Power Plant Structures, Columbia University Technical Report prepared for Brookhaven National Laboratory under Grant No. BNL-517722-S, September 1980. 
40) Mirza, S., MacGregor, J., A Statistical Study of Variables Affecting the Strength of Normal Weight Concrete Members, Structural Engineering Report No. 58, Department of Civil Engineering, University of Alberta, Edmonton, Alberta, Canada, December 1976.

41) National Building Code of Canada, National Research Council of Canada, Ottawa, 1977.

42) Newmark, N. M., Blume, J. A., and Kapur, K. K., Seismic Design Spectra for Nuclear Power Plants, Journal of the Power Division, ASCE, Vol. 99, No. P02, Proc. Paper 10142, November 1973, pp. 287-303.

43) Peir, J. C., and Cornell, C. A., Spatial and Temporal Variability of Live Loads, Journal of the Structural Division, ASCE, Vo1. 99, No. ST5, Proc. Paper 9747, May 1973, pp. 903-922.

44) Rackwitz, R., Practical Probabilistic Approach to Design in First Order Reliability Concepts for Design Codes, Comité Européan du Beton, Bulletin D'Information, No. 112, Munich, West Germany, July 1976.

45) Rafay, T., Structural Design Criteria for Power Plants, Preprint No. 80-044 of the American Society of Civil Engineers, April 1980.

46) Ravindra, M. K. and Galambos, T. V., Load and Resistance Factor Design Criteria for Connectors, J. Struc. Div. Proc. ASCE, 106 (ST9), September 1979, pp. 1427-1441.

47) Reich, M. et al., Review of Methods and Criteria for Dynamic Combinations in Piping Systems, BNL-NUREG/CR-1330, March 1980.

48 ) Seed, H. B. et a1., Site-Dependent Spectra for Earthquake-Resistant Design, Bul1. Seism. Sco. Am. 66, 1976, pp. 221-243. 
49) Shinozuka, M., Probability of Structural Failure under Random Loading, Journal of the Engineering Mechanics Division, ASCE, Vol. 90, No. EM5, October 1964, pp. 147-170.

50 ) Shinozuka, M. and Nishimura, A., On General Representation of a Density Function, Annals of Reliability and Maintainability, Fourth Annual Reliability and Maintainability Conference, Vol. 4 - Practical Techniques and Application, JuTy 28-30, 1965, pp. 897-903.

51) Shinozuka, M., Probability of Structural Failure under Random Loading: Closure to Discussion, Journal of the Engineering Mechanics Division, ASCE, Vol. 92, No. EM1, February 1966, pp. 190-196.

52) Shinozuka, M. and Yao, J. T. P., on the Two-Sided Time-Dependent Barrier Problem, Journal of Sound and Vibration, Vol. 6, No. 1, 1967, pp. 98-104.

53) Shinozuka, M., Itagaki, H. and Hakuno, M., Dynamic Safety Analysis of Multistory Buildings, Journal of the Structural Division, ASCE, Vol. 94, No. ST1, January 1968, pp. 309-330.

54 ) Shinozuka, M., Durability Methods Development, Volume IV: Initial Quality Representation, AFFDL-TR-79-3118, September 1979.

55) Shinozuka, M., Notes on the Combinations of Random Loads (I), Columbia University Technical Report, prepared for Brookhaven National Laboratory under Grant No. BNL-517722-S, July 1980.

56) Shinozuka, M. and Tan, R., Notes on the Combinations of Random Loads (II). Colubmia University Technical Report under BNL-517722-S, August 1980. 57 ) Shinozuka, M., Basic Analysis of Structural Reliability, Columbia University Technical Report, prepared for Brookhaven National Laboratory under Grant No. BNL-517722-S, September 1980. 
58) Simiu, E., Wind Effects on Structures, John Wiley and Sons, New York, 1978.

59) Simiu, E., Changery, M., and Filliben, J., Extreme Wind Speeds at 129

Stations in the Contiguous United States, National Bureau of Standards

Building Science Series, No. 118, March 1979, Washington, D. C.

60) Specifications for the Design, Fabrication and Erection of Steel Buildings, American Institute of Steel Construction, November 1978.

61) Standard Review PTan, NUREG-75/087.

62) Stevenson, J. D., Current Summary of International Extreme Load Design Requirements for Nuclear Power Plant Facilities, Nuclear Engineering and Design, Vor. 60, No. 2, September 1980, pp. 197-209.

63) Thom, H. C. S., Tornado Probabilities, Monthly Weather Review, October-December, 1963, pp. 730-736.

64) Turkstra, C. J., Theory of Structural Design Decisions, Solid Mechanics Study No. 2, University of Waterloo, Waterloo, Ontario, Canada, 1972.

65) Twisdale, L., Tornado Data Characterization and Windspeed Risk, Journal of the Structural Division, ASCE, Vol. 104, No. ST10, October 1978, pp. 1611-1630. 66) Udwadia, F. E. and Trifunac, M. D., The Fourier Transform, Response Spectra and Their Relationship Through the Statistics of Oscillator Response, Report No. EERL 73-01, Calif. Institute of Tech., Pasadena, CA, April 1973.

67) Van Marcke, E., Structural Response to Earthquakes in Seismic Risk and Engineering Decisions (Lomnitz and Rosenblueth, eds.), Elsevier Scientific Publications, 1976.

68) Veneziano, D., Grigoriu, M. and Cornell, C. A., Vector Process Models for System Reliability, J. Engrg. Mech. Div., Proc. ASCE, 103 (EM3), June 1977, pp. 441-460. 
69) Wang, P. C. and Yun, C. B., Site-Dependent Critical Design Spectra, Earthquake Engineering and Structural Dynamics, Vol. 7, 1979, pp. 569-578. $70)$ Wen, Y. K., Chu, S. L., Tornado Risk and Design Wind Speed, Journal of the Structural Division, ASCE, Vol. 99, No. ST12, December 1973, pp. 2409-2421.

$71)$ Wen, Y. K., Statistical Combination of Extreme Loads, J. Struct. Div. Proc. ASCE, 103 (ST5), May 1977, pp. 1079-1093.

72 ) Wen, Y. K., Methods for Reliability of Structures under Multiple Time Varying Loads, J. Nuc. Engrg. and Design, 60, 1980a, pp. 61-71. 73) Wen, Y. K., A Clustering Model for Combination of Correlated Load Processes, Tech. Rep. Dept. of Civil Engrg., University of Illinois, to appear in the J. Struc. Div. Proc. ASCE, 1980b.

74 ) Wen, Y. K., Reliability Analysis Under the Combination of Stochastic Loads, Proceedings of the International Specialty Conference on the Probabilistic Safety of Structures, Paris, France, September 8-9, 1980c. 75) Wen, Y. K., Stochastic Dependencies in Load Combination, to be published in the Proceedings of the 3rd International Conference on Structural Safety and Reliability (ICOSSAR '81), Trondheim, Norway, June 23-25, 1981. 76) Winterstein, S. R., Stochastic Dynamic Response Combinations, M. S. Dissertation, M. I. T., May 1980.

77) Winterstein, S., Distribution of Extreme Value due to Combination of Poisson Square Wave and Impulse Processes, private communication, August 1980. 78) Yang, J-N. and Shinozuka, M., On the First Excursion Probability in Stationary Narrow-Band Random Vibrations, I, J. Applied Mech., Vol. 38, No. 4, Trans. ASME, Series E, 1971. 
Appendix A

Appendix B

Appendix C

Appendix D

Appendix E

Appendix F

Appendix G

Appendix $\mathrm{H}$

Appendix I
Design Methodology for Nuclear Power Plant Structures

Consensus Estimation Procedures

System Reliability Modeling Methods

Second Order Higher Moment Approximation

General Forms of Probability Density Functions

Bounded Tail Probabilities Using Inequalities of the Chebycheff Type

Comments on the Application of Upcrossing Methods to Load Combinations

Notes on the Combinations of Random Loads I

Notes on the Combinations of Random Loads II 


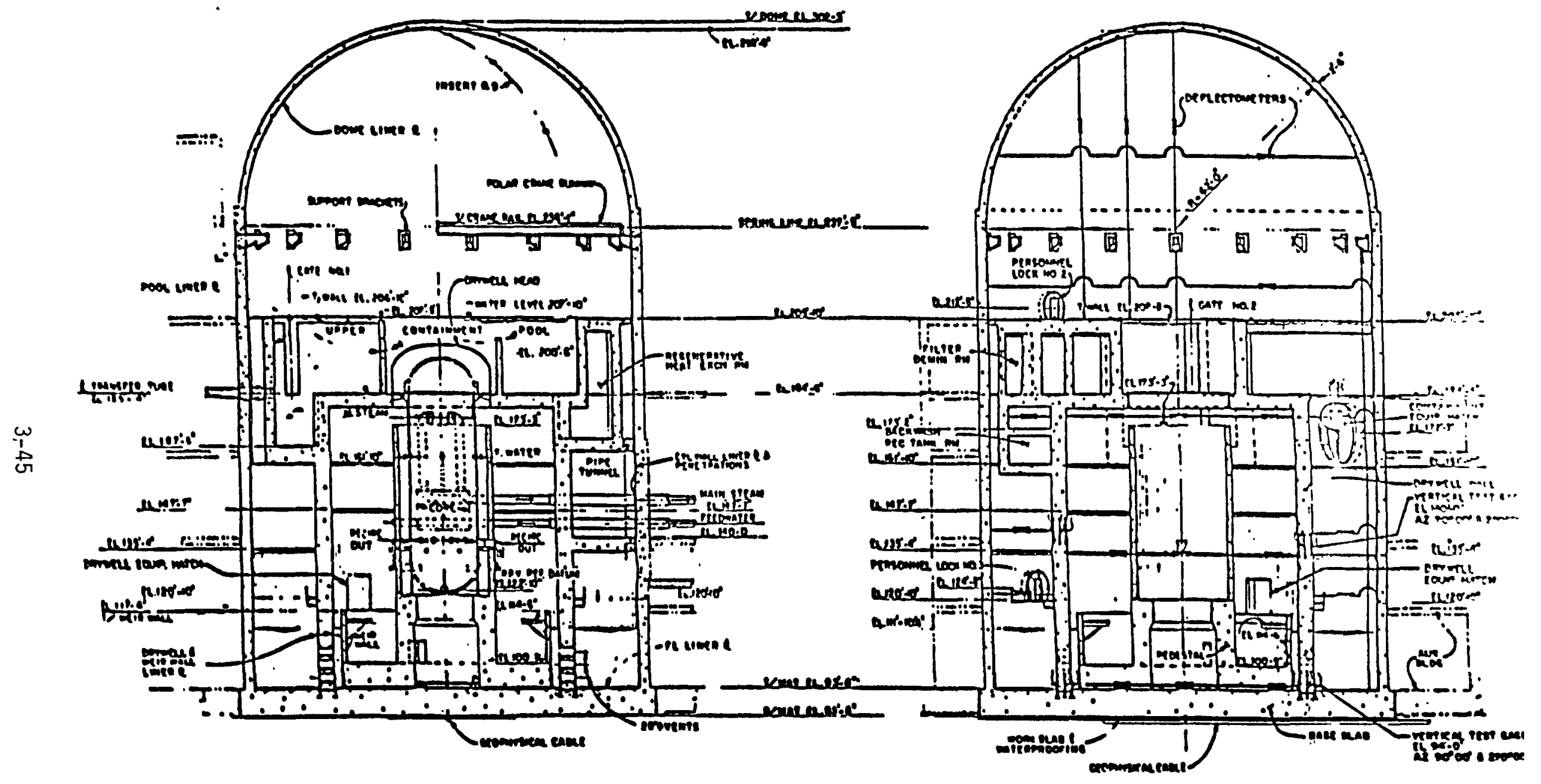

Fig. 3.1 Mark III Containment 
,

$+$ 
Appendix A

Design Methodology for Nuclear Power Plant Structures 
DESIGN METHODOLOGY FOR NUCLEAR POWER PLANT STRUCTURES

by

\author{
Christian Meyer \\ Associate Professor of Civil Engineering \\ Department of Civil Engineering and Engineering Mechanics \\ Columbia University \\ New York, N.Y. 10027
}

September 1980

prepared for

Brookhaven National Laboratory

under Grant No. BNL-517722-S 


\section{INTRODUCTION}

This report summarizes elements of the design process which have some influence regarding the effort of developing a unified load combination methodology. The underlying objective of this report is to examine current code requirements for nuclear safety class structures and to present a brief outline for development of a unified design methodology.

The report is organized according to the following subtasks.

\section{Introduction;}

2. A brief review of the loads of concern for the design of nuclear power plant facilities, and a comment on load combinations;

3. Discussion of structural response characteristics;

4. Current analysis methods;

5. Outline of a unified design methology;

6. Conclusions and recommendations.

Detailed discussions of the reliability theory which forms the basis of modern design methodologies are given elsewhere $(1,2,3)$.

\section{LOADS AND LOAD CATEGORIES}

For metal containments, the pertinent ASME Code Section III, Division ${ }_{1}(4)$ does not define explicitly loads and load categories, but only certain levels of service limits which indirectly correspond to classes of loads or load combinations of increasing severity.

Level A Service Limits - Service loadings to which the containment is subjected, including the plant or system design basis accident 
condition (DBA) for which the containment function is required;

Level B Service Limits - Loads subject to Level A Service Limits plus the additional loads resulting from natural phenomena during which the plant must remain operational;

Level C Service Limits - Loads subject to Level A Service Limits plus the additional loads resulting from natural phenomena for which safe shutdown of the plant is required;

Level D Service Limits - Other applicable service limits and loadings of a local dynamic nature for which the containment function is required.

The code makes reference also to testing conditions in conjunction with hydrostatic or pneumatic tests including leak tests, which normally simulate operating or design basis accident conditions.

For concrete containments, the ASME Code Section III, Division $2^{(5)}$ defines explicit and precise load categories. These are characterized by their sources, increasing magnitude or severity, and decreasing probability of occurrence, or increasing recurrence period. This needs to be determined in terms of the structure design life, which is normally assumed to be thirty to forty years. The load categories are defined as follows:

\section{Construction Category}

Loads in this category include cranes and other equipment loads, scaffolding, wet concrete, prestressing, creep and shrinkage, and wind and are essentially comparable to construction loads in conventional construction. 
2. Test Category

Newly completed containment buildings and reactor pressure vessels have to undergo acceptance testing to verify structural integrity and leak tightness. The loads to be considered are an initial proof test pressure, $P_{t}$, and the test temperature, $T_{t}$. The significance of these loads is that their magnitudes are well-controlled while intended to simulate normal operating and design basis accident conditions.

3. Normal Load Category

This category includes all loads due to plant start-up, normal operation and scheduled shutdown for refueling and maintenance service. These are:

D - dead loads, including hydrostatic and permanent equipment loads

$L$ - live loads, including any moveable equipment loads and other loads which vary with intensity and occurrence (e.g., soil pressure)

F - loads resulting from the application of prestress

$T_{0}$ - thermal effects and loads during normal operation or shutdown condition, based on most critical transient or steadystate condition

$R_{0}$ - pipe reactions during normal operation or shut-down condition, based on most critical transient or steady-state condition

$P_{v}$ - external pressure loads resulting from pressure variation either inside or outside the containment.

In addition, concrete creep and shrinkage and prestress relaxation are loads to be considered in this category. 


\section{Abnormal Load Category}

This load category covers all loads generated by the design basis accident (DBA) or loss-of-coolant accident (LOCA) and other stipulated accident conditions involving high energy pipe ruptures.

These loads include specifically

$T_{a}$ - thermal effects and loads, generated by the DBA, including $T_{0}$ $R_{a}$ - pipe reactions from thermal conditions, generated by the DBA, including $R_{0}$

$R_{r}$ - local effects on the containment due to DBA, including $R_{r r}$ (or $Y_{r}$ ) - load generated by the reaction of a ruptured high energy pipe, including dynamic load factor

$R_{r j}$ (or $Y_{j}$ ) - load generated by jet impingement from a ruptured high energy pipe, including dynamic load factor $R_{r m}$ (or $\left.Y_{m}\right)$ - load resulting from impact of a ruptured high energy pipe, and other internally generated missiles

$H_{a}$ - load resulting from internal post-LOCA flooding, if such an occurrence is defined in the design specification.

It may be noted that for concrete reactor vessels, abnormal loads are also defined as loads which will not result in structural damage requiring repair (1980 version), while in the 1975 edition the loads were defined as those which require immediate corrective action or orderly shutdown of the plant. Also to be considered is tendon failure or material degradation equal to the total design prestress loss, e.g., $-0.15 \mathrm{~F}$. 
5. Severe Environmental Load Category

Loads in this category are those to be encountered "infrequently" during the plant life, i.e., loads to occur once in a while, such as

W - loads generated by the design wind specified for the plant site (OBW)

$E_{0}$ - loads generated by the operating basis earthquake (OBE).

6. Extreme Environmental Load Category

This category is defined to contain loads to be considered "credible but highly improbable," i.e., loads of such large recurrence periods that the probability is small that the structure will be subjected to them during its lifetime. These loads include $E_{S S}$ - loads generated by the safe shutdown earthquake (SSE) $w_{t}$ - tornado loading including the ef fects of missile impact, including

$W_{t q}$ - loads due to tornado wind pressure

$W_{t p}$ - differential pressure loads due to atmospheric pressure change

$W_{t m}$ - tornado generated missile impact effects

For either loading condition, the plant is required to respond such as to permit safe shutdown. Other loads not specifically mentioned in the code but in the USNRC Standard Review Plan ${ }^{(6)}$ include tsunami, aircraft impact, gas explosion, probable maximum flood, etc., which are to be considered on a case-by-case basis.

7. Failure Category

This load category is required by the current code ${ }^{(5)}$ explicitly 
only for concrete reactor vessels (CRV). Failure category load combinations would normally be the result of postulated component failures of extremely remote possibility, such as $P_{m c}$ - pressurized crack condition

$T_{f}$ - temperature increases in mass concrete of the CRV where concrete structural capacity is required

$Y_{j r}$ - steam impingement on prestressing anchorage assemblies

$-\frac{3}{2} F$ - tendon failure or material degradation up to one-half of design basis value

and penetration closure failure. The design requirement for any loading condition of this category is that the structure shall not fail.

The above load categories are much more specific than the corresponding service level limits for metal containments.

Precise data as for the actual values to be used in each load category are difficult to prescribe because the data base for the above-mentioned loads is considerably sparser than the corresponding body of knowledge regarding loads in conventional construction. A summary of the state of knowledge of actual nuclear power plant loads is given in Ref. (14).

For metal containments, the code ${ }^{(4)}$ requirements for load combinations are vague, in effect leaving it up to the Standard Review Plan ${ }^{(6)}$ to interpret them and to define load combinations which are acceptable to the NRC. For concrete containments, the code ${ }^{(5)}$ is quite explicit, defining load categories summarized in Table 1. It is noteworthy that the combination of environmental and abnormal loads implies full correlation, as if a DBA could be the result or consequence of environmental loads. 


\section{STRUCTURAL RESPONSE}

The widely varying recurrence periods of loads assoclated with the different categories require different response categories or levels of performance. In current design practice, one may distinguish three different response levels: service response level, factored load response level, and failure load conditions.

\section{Service Load Response Level}

Under construction, test, normal and severe environmental category loads, structures are expected to respond linear-elastically, with stresses well below the allowable values specified in the codes. Implied in this response level or performance criterion is the requirement that the structure ensure safe operation of the plant without damage, and that deformations remain within tolerances which permit safe functioning of equipment. For concrete reactor vessels, the code wording is clear enough to make normal, abnormal and severe environmental load categories comparable to the Level A and B Service Limits for metal containments.

\section{Factored Load Response Level}

Factored loads are mentioned only in the code for concrete vessels and containments $^{(5)}$ and are comparable to the factored loads used in Ultimate Strength Design according to the ACI-Code ${ }^{(15)}$. For all abnormal and extreme environmental category loads or factored loads, structures are expected to respond essentially linear-elastically, with stresses below allowable values specified in the codes. Excepted are loads due to DBA and tornado missile impact, for which the structure's ability to deform beyond yield may be considered. Design adequacy is assured by limiting the permissible ductility 
demand.

For concrete reactor vessels, the Code wording permits the conclusion that the extreme environmental load category is equivalent to Level C Service Limit for metal containments. Load effects in this category shall not exceed three-quarters of the ultimate strength of a critical section. In any case shall the structure response permit the safe shutdown of the plant.

\section{Failure Load Condition}

Failure load conditions for metal containments are applicable in conjunction with plastic analysis $(4)$, in which case the specified loading shall not exceed two-thirds of the lower bound collapse load, i.e., the implied safety factor is 1.5. The code for concrete vessels and containments ${ }^{(5)}$ makes explicit reference to failure category loads only in conjunction with concrete reactor vessels. This appears to be an inconsistency of the code, because the assessment of the factor of safety against structural failure is no less important for nuclear containment structures than for conventional concrete construction, even though margins of safety against exceeding serviceability criteria may be just as important. In containment design, we therefore have to distinguish between functional and structural failure modes. The primary functional failure mode consists of the uncontrolled release of radioactivity. Structural failure modes may consist of any of the following: liner breakage, buckling, local buckling, local penetration failure, flexural failure, shear failure, explosive compression failure of prestressed concrete.

\section{ANALYSIS METHODS}

The various methods available for the analysis of nuclear containments 
are well-documented in the 1iterature. In particular, Refs. 10, 11, 12 contain useful summaries of pertinent analysis techniques. Here, only the Code requirements shall be summarized briefly, as they apply to steel containments, concrete containments and concrete reactor vessels.

\section{Steel Containments}

An accurate stress analysis is required for all load combinations. As an alternative, the code ${ }^{(4)}$ permits design by formula. In Appendix A, formulas and methods of solution are given for certain recurring problems. Accuracy and reliability of the results and analys is cost strongly favor analys is by computer. Structural analysis programs are available to analyze arbitrary structures by the finite element method as well as axisymmetric shell structures for which nonsymmetric loads are resolved into Fourier series. It is important that programs are thoroughly verified before use, following, for example, the procedure described in the Standard Review Plan (6).

The following types of analysis may be required for metal containments.

- Linear static analysis. This is adequate for most Level A and B Service Limit States.

- Linear buckling analysis.

- Linear Dynamic Analysis. Time history or frequency response or response spectrum analysis is required to compute the load effects of environmental loads (wind, earthquake, tornado), including soilstructure interaction, and the local effects of Design Basis Accidents, unless "appropriate" dynamic amplification factors are used.

- Nonlinear dynamic analysis is accepted by the NRC for checking local buckling under localized pressure of the Design Basis Accident.

- Plastic analysis and shakedown analysis are code allowable alterna- 
tives to linear stress analysis for assessing the structure's strength reserve against failure.

\section{Concrete Containments}

In a popular "catch-all" phrase, the code ${ }^{(5)}$ requires that "methods of analysis which are based on accepted principles of engineering mechanics and which are appropriate to the geometry of the containment shall be used." Specifically to be considered are the redistribution of moments and forces in statically indeterminate structures because of concrete cracking and the stiffening effect of buttresses and other integral portions of the containment structure; short and long term fourdation soil properties; concrete creep and thick section geometry, in the case of prestressed concrete, but limited to construction and normal category loads; sensitivity of analysis results to material property changes, for which upper and lower bounds are required.

Linear elastic behavior is an acceptable assumption for stress analysis, partially as a necessity, since the state of the art of nonlinear analysis of concrete structures is not sufficiently advanced for common use in engineering practice ${ }^{(16)}$

The analysis of the steel liner represents a special case. Being a metal component of a concrete structure, the analysis and design requirements are confused by the inconsistency of the governing codes, viz. the service level states of metal containment design and ultimate strength of concrete design. All capacities of components are specified in terms of stress, strain, force or displacement, whichever is applicable. Since many of the allowable capacities are based on ultimate capacities, testing of a prototype may be necessary to verify the ultimate capacity of a particular part. 


\section{Prestressed Concrete Reactor Vessels}

A three-dimensional linear elastic analysis is adequate for construction and normal category as well as abnormal and severe environmental category loads. Effects of long term creep and shrinkage also have to be accounted for.

Extreme environmental and failure category loads may have to be assessed through model tests, unless analysis methods are used which predict with reasonable accuracy the ultimate load behavior of the concrete reactor vessel under investigation, using the total stress-strain curves for all materials.

The extraordinary difficulties of analyzing the nonlinear behavior of concrete structures are well known. Together with the uncommon computational expenses, they have been responsible for the fact that such complete analyses are not employed routinely in engineering practice. The state of the art of nonlinear finite element analysis of concrete structures has been compiled recently by an ASCE task committee formed for the explicit purpose of producing this state of the art report ${ }^{(16)}$. Applications of this advanced technology are at present restricted to mostly academic structural elements, while the analysis of realistic engineering structures is relatively scarce ${ }^{(17)}$.

5. UNIFIED DESIGN METHODOLOGY

It is possible to group the basic design philosophies into the following categories.

1. Allowable stress design, which satisfies serviceability criteria but results in uncertain or nonuniform factors of safety;

2. U7timate strength design, which produces consistent factors of safety against failure, but does not directly satisfy serviceability re- 
quirements;

3. Load and resistance factor design ${ }^{(9)}$, which attempts to combine the previous two approaches by satisfying a series of both service level and ultimate limit states with a consistent level of reliability. Structures are designed to serve certain functions in an acceptable way. When a structure or structural element ceases to serve its function or intended purpose, it is said to have reached a limit state. Such 1 imit states are by definition of a discrete nature. The collapse of a beam, the breakage of a window, or the leakage of a liquid storage tank are examples, whereas the exceedence of an allowable stress is not a limit state, because "nothing happens."

Serviceability limit states are related to the disruption of functional use, damage or deterioration of a structure. Since exceedence of such 1 imit states may obliterate the primary purpose of the structure's existence, the probability of occurrence should be kept as small as feasible. Where the exceedence is reversible, for example through repair, the probability of occurrence need not be as low as otherwise required.

Ultimate limit states are related to the safety of a structure, such as complete or partial collapse. The probability of such an occurrence should be kept as small as possible.

Examples of ultimate 1 imit states are on the structural level: overturning, uplift, sliding, global instability, progressive collapse and flutter. On the element level, examples are: fracture, cracking, crushing and buckling.

In the case of nuclear containment buildings, the uncontrolled release of radioactivity is a catastrophe of such severity that it should be con- 
sidered an ultimate limit state comparable to collapse, the probability of occurrence of which should be kept as small as possible.

Examples of serviceability limit states are: excessive deflections affecting the appearance, functional use, drainage, or causing damage to non-structural components and their attachments; excessive local damage such as cracking, splitting, spalling, local yielding and slip, which affect the appearance, use or durability of the structure; excessive vibrations affecting the comfort of occupants or the operation of equipment.

The most comprehensive description of probability-based limit states design methodology or load and resistance factor design (LRFD) can be found in Ref. 9 on which much of this summary is based.

The LRFD design format and methodology can be considered to combine three distinct phases.

1. Identification of all modes of fallure or ways in which the structure might fail to serve its intended purposes;

2. Determination of acceptable levels of safety aga inst occurrence of each limit state;

3. Consideration by the designer of all significant 1 imit states, as prescribed by regulatory agencies such as the NRC.

Although developed for non-nuclear building code requirements, this design methodology offers various advantages also for nuclear construction. However, certain 1 imit states peculiar to nuclear safety class structures deserve special attention, which could not be considered in Ref. 9.

The advantages of probability-based limit state design are well documented in the literature (see Ref. 9 for further references) and are here summarized for completeness. 
1. The method achieves a more consistent reliability for different design situations than other methods, because the variabilities of various loads and strengths are considered explicitly and independently.

2. The reliability level can be chosen to reflect the consequences of failure.

3. The designer will achieve a better understanding of the fundamental structural requirements and of the structural behavior in meeting those requirements.

4. The design process is simplified by the adoption of a uniform design philosophy and procedures applicable for different materials of construction.

5. The design method provides a rational tool for exercizing judgment in non-routine situations.

6. It also provides a tool for updating standards in a rational manner. It is worthwhile to note that the substitution of ultimate strength design for working stress design of concrete structures has el iminated certain unjustified conservatisms and consequently has led occasionally to savings in cost. This factor was not the least among those that helped expedite the transition within approximately ten years.

When replacing current design practice by LRFD, no such savings in cost will result, if the target reliabilities are calibrated against the reliability levels currently in use -- as is done in Ref. 9. This will apply to both conventional as well as nuclear construction. In other words, the primary objective of LRFD is not a reduction in cost, but the achievement of a uniform and consistent level of reliability. 


\section{CONCLUSIONS AND RECOMMENDATIONS}

The purpose of this report is to view the problem of "Load Combinations for Design of Seismic Category I Structures" in a proper perspective, namely as a subproblem of developing a unified load combination methodology for nuclear power plant structures. Questions of loads, load factors and load combinations refer only to some aspects of the wider problem of a consistent and rational design methodology. The recognition of some inconsistencies in the current codes should also be viewed in this sense, namely as another aspect of the overall problem which can be improved when implementing a probability-based limit states design procedure with load and resistance factor design (LRFD) format. The following observations can be made in this regard.

1. The different authorship of Division 1 and Division 2 of the ASME Code, Section III $(4,5)$ is obvious, reflecting the historical division of the engineering profession into groups adhering to either the working stress design concept or to ultimate strength and $11 \mathrm{~m}-$ it states design philosophy.

2. The resulting differences between the codes can be removed if a unified design concept is introduced.

3. Current lists of load combinations (such as Table 1) exhibit an element of arbitrariness. Rigorous analysis can be used to derive rational sets of load cases and load combinations.

4. Currently used load factors (see, e.g., Table 1) appear to be inconsistent compared with the load factors common in ultimate strength provisions for conventional structures. For example, the load combination "normal plus extreme environmental" as listed 
in Table 1 has only unit load factors, $1.0\left(D+L+F+T_{0}+R_{0}+\right.$ $\left.P_{v}\right)+1.0\left(E_{s S}\right)$ although the concrete compressive stress value not to be exceeded for primary plus secondary membrane and bending stress is $0.85 \mathrm{f}_{c}^{\prime}$. i.e., the conventionally assumed ultimate concrete compression strength. The comparable load case for conventional buildings according to the ACI-Code $e^{(15)}$ is $0.75(1.4 D+1.7 \mathrm{~L})$ $+1.4(E)$.

5. For steel containments, neither explicit load combinations nor load factors are given in the Code. Therefore the Standard Review Plan provides an interpretation of the Code's intent by defining load combinations and load factors acceptable to the NRC. In the case of plastic analysis, the code-prescribed factor of safety against failure of 1.5 needs to be examined.

6. The code discrepancies between metal and concrete containment design provisions may cause complications for the design of the steel liner; this item needs further evaluation.

7. A unified design methodology needs to be defined for all types of materials.

8. For design checks involving serviceability limit states, linear elastic analysis methods may be adequate.

9. For design checks involving ultimate limit states, analysis methods should be used which are capable of determining the ultimate strength of the structure and its components.

10. The statistical data base of nuclear power plant loads is small compared with that available for conventional construction. Efforts should be undertaken to broaden this data base. 
11. Quality assurance and quality control of nuclear construction far exceeds the standards common in conventional construction. Statistical uncertainties of resistances are therefore smaller.

12. The probability of simultaneous occurrence of different loads has to be investigated, in particular the hypothetical case of abnormal load cases triggered by environmental loads such as wind or earthquake.

13. Acceptable levels of target reliabilities have to be determined.

14. When establishing acceptable target reliabilities, the consequences of failure require special consideration.

15. The reliability analysis such as is utilized in Ref. 9 has been applied in the past only to single structure components. The analysis techniques need to be extended also to the structure system reliability.

16. It is felt that any increases in engineering cost due to the introduction of a uniform design methodology are likely to be marginal relative to construction costs or cost increases caused by delays in the licensing process.

There are other items of concern, for example human or socio-economic factors. However, these are beyond the scope of the current effort and thus are not included in this report. 
CC.3222.2-CC.3222.3 SECTION III. DIVISION 2 - SUBSECTION CC

TABLE CC-3230-1

LOAD COMBIMATIONS ANO LOAD FACTORS

\begin{tabular}{|c|c|c|c|c|c|c|c|c|c|c|c|c|c|c|c|c|c|}
\hline Cmesong & 0 & $l^{\prime}$ & F & $n_{1}$ & P. & $r_{1}$ & 8 & 1. & 6 & $E_{00}$ & $w$ & $w_{1}$ & B & $R_{0}$ & $a_{0}$ & $\theta_{0}$ & $M_{0}$ \\
\hline \multicolumn{18}{|l|}{ Sentice } \\
\hline Text & 2.0 & 1.0 & 1.0 & 1.0 & $\cdots$ & 1.0 & $\cdots$ & $\cdots$ & $\cdots$ & $\cdots$ & $\cdots$ & $\cdots$ & $\cdots$ & $\cdots$ & $\cdots$ & $\cdots$ & $\cdots$ \\
\hline Conservetion & 1.0 & 1.0 & 1.0 & $\cdots$ & $\cdots$ & $\cdots$ & 1.0 & $\cdots$ & $\cdots$ & $\cdots$ & 1.0 & $\ldots$ & ... & $\cdots$ & $\cdots$ & $\cdots$ & $\ldots$ \\
\hline Normel & 1.0 & 1.0 & 1.0 & $\cdots$ & .. & $\cdots$ & 1.0 & $\cdots$ & $\cdots$ & $\cdots$ & $\cdots$ & $\cdots$ & 1.0 & $\cdots$ & $\cdots$ & 2.0 & ... \\
\hline Severe entronmement & $\begin{array}{l}1.0 \\
1.0\end{array}$ & $\begin{array}{l}1.0 \\
1.0\end{array}$ & $\begin{array}{l}1.0 \\
1.0\end{array}$ & $\ldots$ & $\ldots$ & $\cdots$ & $\begin{array}{l}1.0 \\
1.0\end{array}$ & $\cdots$ & $\begin{array}{l}1.0 \\
\ldots\end{array}$ & $\cdots$ & $\dddot{i .0}$ & $\cdots$ & $\begin{array}{l}1.0 \\
1.0\end{array}$ & 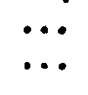 & ... & $\begin{array}{l}1.0 \\
1.0\end{array}$ & 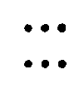 \\
\hline
\end{tabular}

Factored

Senere environmemal

$\begin{array}{lllllllllllllllll}1.0121 & 1.3 & 1.0 & \ldots & \ldots & \ldots & 1.0 & \ldots & 1.5 & \ldots & \ldots & \ldots & 1.0 & \ldots & \ldots & 1.0 & \ldots\end{array}$

$\begin{array}{llllllllllllllllll}1.0121 & 1.3 & 1.0 & \ldots & \ldots & \ldots & 1.0 & \ldots & \ldots & \ldots & 1.5 & \ldots & 1.0 & \ldots & \ldots & 1.0 & \ldots\end{array}$

Entreme entronmemeat

$\begin{array}{llllllllllllllllll}1.0 & 1.0 & 1.0 & \ldots & \ldots & \ldots & 1.0 & \ldots & \ldots & 1.0 & \ldots & \ldots & 1.0 & \ldots & \ldots & 1.0 & \ldots\end{array}$

$\begin{array}{llllllllllllllllll}1.0 & 1.0 & 1.0 & \ldots & \ldots & \ldots & 1.0 & \ldots & \ldots & \ldots & \ldots & \ldots & 1.0 & 1.0 & \ldots & \ldots & 1.0 & \ldots\end{array}$

abnoment

$\begin{array}{lllllllllllllllll}1.0 & 1.0 & 1.0 & \ldots & 1.5 & \ldots & \ldots & 1.0 & \ldots & \ldots & \ldots & \ldots & \ldots & 1.0 & \ldots & \ldots & \ldots\end{array}$

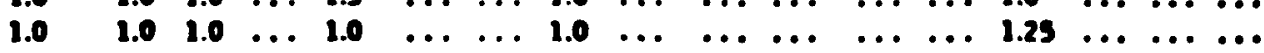

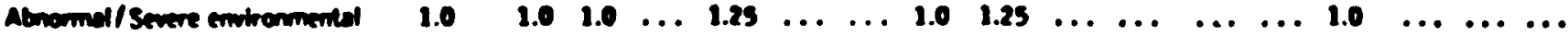

$\begin{array}{lllllllllllllllllll}1.0 & 1.0 & 1.0 & \ldots & 1.25 & \ldots & \ldots & 1.0 & \ldots & \ldots & 1.25 & \ldots & \ldots & 1.0 & \ldots & \ldots & \ldots\end{array}$

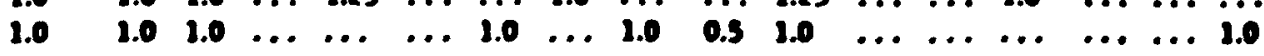

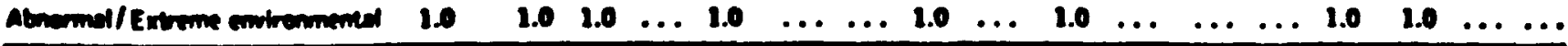

mores:

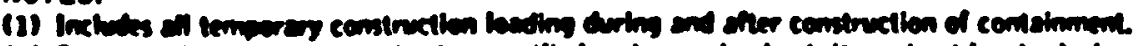

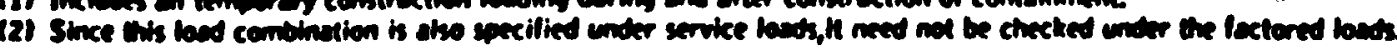




\section{REFERENCES}

1) Shinozuka, M., "Notes on the Combinations of Random Loads (I)," prepared for the Brookhaven National Laboratory under Grant No. BNL-517722-S, July 1980.

2) Shinozuka, M. and Tan, R., "Notes on the Combinations of Random Loads (II)," prepared for the Brookhaven National Laboratory under Grant No. BNL-517722-S, August 1980.

3) Shinozuka, M., "Basic Analys is of Structural Safety," prepared for the Brookhaven National Laboratory under Grant No. BNL-517722-S, September 1980.

4) ASME Boiler and Pressure Vessel Code, Section III, Division 1 (1980).

5) ASME Boiler and Pressure Vessel Code, Section III, Division 2 (1980).

6) USNRC, Standard Review Plan, Sections 3.8.1 through 3.8.4, (1975).

7) USNRC, Regulatory Guide 1.57, "Design Limits and Loading Combinations for Metal Primary Reactor Containment System Components," (1973).

8) "Code Requirements for Nuclear Safety Related Concrete Structures," ACI Standard 349-376 (1976 with 1979 supplement).

9) Ellingwood, B., Galambos, T. V., MacGregor, J. G. and Corne11, C. A., "Development of a Probability Based Load Criterion for American National Standard A58," NBS Special Publication 577, 1980.

10) Chou, C. K. et al., "Seismic Safety Margins Research Program, Load Combination Project Task 3, Load Combination Methodology Development, Interim Report .I," Lawrence Livermore Laboratory, January 1980.

11) Seide, P., Weingarten, V. and Masri, S., "Buckling Criteria and Application of Criteria to Design of Steel Containment She11," USNRC, Report CR-0793, May 1979. 
12) Healey, J. J., Wu, S. T. and Murga, M., "Structural Building Response Review, Seismic Safety Margins Research Program," (Ebasco), USNRC, Report CR-1423, Vol. I.

13) Singh, A. K., Hsu, T. I. and Khatua, T. P., "Structural Building Response Review, Seismic Safety Margins Research Program," (Sargent \& Lundy), USNRC, Report CR-1423, SL-3759, Vol. II.

14) Chiu, K. D. and Ellingwood, B. - Report to BNL.

15) "Building Code Requirements for Reinforced Concrete," American Concrete Institute Standard 318- 77, Detroit, Michigan, 1977.

16) "Finite Element Analysis of Reinforced Concrete Structures," State-ofthe-Art Report, Task Committee on Finite Element Analys is of Reinforced Concrete Structures, ASCE, Special Publication, in press.

17) Meyer, C. and Bathe, K. J., "Nonlinear Analys is of Concrete Structures in Engineering Practice," Journal of the Structural Division, ASCE, in press. 
Appendix B

Consensus Estimation Procedures 


\section{Introduction}

In the course of this work it has been found that the collection of sufficient, detailed load dıta is a difficult task. Sometimes hard, quotable data is not available for a variety of reasons such as:

(a) Load data is improperly recorded or not recorded at all.

(b) Data is of a proprietary nature and cannot be released.

(c) Newness of concept or design prevents one from having a recorded history of field experience.

(d) Data samples occur with such a low frequency that many years (decades) are required to obtain significant data.

(e) Load data may be well known for an initial design, however, the changes in the final design or during construction may not be recorded and anlyzed.

Initiation of new data collection programs are often not feasible, because of the long time duration and high cost of such procedures.

As an aid in providing initial approximate numerical values where no data exists, the consensus approach is recommended $[1,2]$.

2. Consensus Estimation Techniques

The consensus approach is really just an averaging of intelligent guesses by experts based on experience and partial data. In fact, sometimes actual proprietary data is available, and can only be furnished if it is reported as an "expert guess".

In this appendix we will discuss analytical consensus, meaning a systematic approach involving careful descriptions of the assumptions about the experts and the resulting choice of statistical approach used in analyzing their opinions. This technique is different from, but has some of the same flavor as the technique used for estimation of prior densities in Bayesian Analysis and the Delphi Technique for forecasting ${ }^{[3,4]}$. 
In the consensus approach we ask a panel of experts to give their estimates (guesses) for the basic probabilities or failure rates for a well described system. The correctness of the results depends upon the knowledge and unbiasedness of the experts and the size of the sample (panel). The results based on the first round of the estimation can be fed back to the experts with the request that they provide revised estimates. If no names are used in the distributed results, some experts who are unsure of themselves and have guessed very high or very low may tend to revise their guesses closer to the mean of the group. If names are released, and one of the experts is well known and respected in the field, his opinions may dominate the second round.

To control the accuracy of the end results, the experts may be asked to rate their expertise on a suitable scale depending upon whether the guess is backed up by hard data, partial data, data on similar products, etc.

\section{Treatment of the Data}

The expert may be requested to give a point estimate, a range estimate (minimum and maximum) or a three-point estimate (minimum, maximum and mean).

The estimates of the experts are then combined using a choice of statistical techniques:

(1) Maximum Likelihood Estimation (MLE)

(2) An Unbiased Linear Combination (ULC)

(3) A Linear Unbiased Minimum Variance (LUMV) estimator.

(4) Preanalysis of the data to determine if clustering is present (e.g., separate groups of optimists and pessimists) and segregation of the data into homogeneous groups. Subsequently, one of the above estimation techniques is applied to each group separately. 
All these techniques result in relatively simple formulae which are presented along with the mathematical development in Reference 1.

4. Choice of the Experts 5

It is very important to exercise good judgement in the choice of experts. First of al1, the experts must be knowledgeable in the subject area. If the experts know little more about the quantitative values to be estimated then a random selection of the general public, the results of the method are of no value.

5. Preparation of the Questionnaire

In performing a consensus estimate it is valuable to meet with a pilot group before a consensus estimation questionaire is prepared. Many of the terms and concepts which seem obvious to the preparer will be misinterpreted by the experts, since each may have his own terminology and viewpoint. In fact one of the prime benefits of a multistage consensus estimate is to allow written and oral (in person or by telephone) comments and discussion between the preparer and the experts. As an example, suppose we ask a group of experts to estimate the wind velocity which modern office buildings must withstand. We might expect a group of experts to give the following hypothetical responses if given no further direction:

(a) Expert 1: The answer is 200 miles per hour, since that is the highest hurricane wind velocity recorded in the U. S.

(b) Expert 2: The answer is 25 miles per hour, since data recorded at the New York City World Trade Center shows a weekly wind gust maximum which averages 25 miles per hour.

(c) Expert 3: The answer is 50 miles per hour since Building Code $X Y Z$ tells us to design for 50 miles per hour. 
(d) Expert 4: The minimum value is 25 miles per hour, the average value is 50 miles per hour, and the maximum is 75 miles per hour. This response is based on the mean wind velocity recorded in Chicago, $\mu=50$ miles per hour, and the upper and lower $2 \sigma$ values of 25 and 75 miles per hour.

In actuality, the response of the 4 th expert is probably the information which was desired. This example indicates the difficulty and desirability of properly describing the quantities to be estimated. The use of min, most.likely, and $\max$ values along with proper definitions aids in properly describing the data sought. 


\section{References}

1) M. L. Shooman and S. Sinkar. "The Use of Consensus in Analytical Safety", Poly-EE-77-305/SMART 105, Polytechnic Institute of New York, February 1977.

2) M. L. Shooman and S. Sinkar. "Generation of Reliability and Safety Data by Analysis of Expert Opinion", Proceedings of 1977 Annual Reliability and Maintainability Symposium, IEEE, New York, pp.186-193.

3) H. Sackman and L. Books. "Delphi Critique", D. C. Heath and Co., Lexington, Massachusetts, 1975.

4) N. Dalkey and 0. Helmer. "An Experimental Application of the Delphi Method to the Use of Experts", Management Science, April 1963, pp. 458-467.

5) IEEE Std. 500-1977, "IEEE Guide to the Collection and Presentation of Electrical, and Sensing Component Reliability Data for Nuclear Power Generating Stations", IEEE, New York, 1977, pp. 25-30. 
Appendix C

System Reliability Modeling Methods

C-1 


\section{Introduction}

For very complex systems it is necessary to develop probabilistic methods which help one to formulate the system reliability in terms of probabilities of occurrence or non-occurrence of the physical events. These events represent limited pieces of the system or procedures and thus, decompose the system into smaller entities which are easier to analyze. The concept of decomposition is sometimes spoken of as the "divide and conquer" strategy.

The choice of which set of events best facilitates system decomposition is treated in sections 4 and 5 . In sections 2 and 3 we only deal with the techniques which aid us in writing the reliability expression for the system in terms of the chosen set of events. Mathematicallv we can describe these techniques as being either expansion into mutually exclusive events or cut-set and tie-set methods.

\section{Mutually Exclusive Events Method}

One way of computing the reliability of the system is to define a set of mutualiy exclusive events, $E_{i}$, which represent the success of the system.

$$
R=P\left(E_{1}+E_{2}+\ldots \ldots \ldots+E_{i}\right)
$$


Since the events are mutually exclusive, Equation 1 simplifies into a simple sum of probabilities (1).

$$
R=P\left(E_{1}\right)+P\left(E_{2}\right)+\ldots \ldots+P+P\left(E_{i}\right)
$$

If we focus on a set of mutualiy exclusive failure events, $\bar{E}_{j}$, we obta in a similar set of equations:

$$
\begin{aligned}
& R=1-P F=1-P\left(\bar{E}_{1}+\bar{E}_{2}+\ldots . \bar{E}_{j}\right) \\
& R=1-P\left(\bar{E}_{1}\right)+P\left(\bar{E}_{2}\right)+\ldots+P\left(\bar{E}_{j}\right)
\end{aligned}
$$

Equations 1 - 4 are simple enough, however, the difficulties arise because there are many events and it is difficult to enumerate a mutually exclusive set. Two engineering approaches have been used to aid in this process and are generally called the Failure Modes Effects and Criticality Analysis (FMECA) and the Event Tree Method (ETM). ${ }^{(1,2)}$

\section{Cut-Set and Tie-Set Methods}

These methods obtain their name from the fact that the mathematical principles underlying the method can be best explained in terms of graph theory. Success is related to connection of the network which is determined by the graph tie-sets. Similarly, failure is related to disconnection of the network which is determined by the network cut-sets.

One approach to modeling the system is the so-called reliability block diagram (RBD) or reliability graph approach. ${ }^{(3)}$ In this case the physical problem is modeled by various success paths such that proper operation of all of the elements on any one success path results in success. The collection of the success paths are the tie-sets of the network.

For example, suppose that there are 3 tie-sets, $T_{1}, T_{2}$, and $T_{3}$. Then the system reliability expression is given by 


$$
R=P\left(T_{1}+T_{2}+T_{3}\right)
$$

where the symbol " + " inside the parenthesis stands for union.

Expansion of Equation 5 yields

$$
\begin{aligned}
R= & P\left(T_{1}\right)+P\left(T_{2}\right)+P\left(T_{3}\right) \\
& -P\left(T_{1} \cdot T_{2}\right)-P\left(T_{1} \cdot T_{3}\right)-P\left(T_{2} \cdot T_{3}\right) \\
& +P\left(T_{1} \cdot T_{2} \cdot T_{3}\right)
\end{aligned}
$$

where the symbol "." inside the parentheses stands for intersection. Note that if they are $\mathrm{N}$ tie-sets, the expanded equation for the reliability contains $\left(2^{N}-1\right)$ terms. To deal with this complexity, it is common to use computer programs and to base these on various bounds and approximations $(2,3)$.

A similar approach which focuses on the probability of failure is the fault tree method. ${ }^{(4)}$ The method begins by defining failure as the top event. Immediately below the top event we draw a logic "or" gate and the inputs to the gate are the various modes of system failure. Each failure mode is expressed in terms of other elementary events and modeled by various logic "and" "or" combinations which are represented by combinations of logic gates and events. The process continues until only the elementary set of component or subsystem events appear as inputs. The diagram then looks like an uprooted, inverted tree.

The set of elements which represent a system failure is called a cut-set of the fault tree. If we assume that in a particular example there are 4 cut sets, $C_{1}, C_{2}, C_{3}, C_{4}$, then we can write

$$
R=1-P_{f}=1-P\left(C_{1}+C_{2}+C_{3}+C_{4}\right)
$$


The expansion of Equation 7 requires many terms and proceeds in a manner similar to Equation 5. If there are in general $\mathrm{K}$ cut-sets, then the expansion involves $2^{\mathrm{K}}$ terms. Computer programs and approximations are used in the same way as for reliability block diagrams.

In general the choice among FMECA, ET, RBD, and FT methods is based upon the complexity of the problem, the experience and personal preferences of the analyist with one method or the other, and the availability of computerized tools. In the case of structural systems other considerations are important and force the use of one modeling technique over others. 


\section{Difficulties in Analysis of Structural Systems Reliability}

All the system reliability analysis methods discussed above are based upon decomposition of the structure into simpler structures. This is easy enough to do conceptually with structural systems, however, the statistical dependices among the various elements of the system make the computation quite complex. A simple example clearly illustrates these concepts.

Consider the simplified model of a nuclear reactor containment structure given in Figure 1. If we assume that we are inside the containment vesser looking outward, then the system of interest is composed of:

1. A reinforced concrete containment cylinder.

2. A reinforced concrete spherical dome.

3. A steel cylindrical liner for the containment cylinder.

4. A concrete drywell structure, modeled by another cylinder.

It is easy to speak of these four components as elements of the system. In fact we can denote them as $x_{1}, x_{2}, x_{3}$ and $x_{4}$ where the $x$ events mean success of that component. Since all the components must succeed for the containment system to succeed, we can make a simple series RBD model for this system which has one tie-set $x_{1} x_{2} x_{3} x_{4}$. In other words, all elements must succeed for the system to succeed. The reliability expression is:

$$
R=P\left(x_{1} \cdot x_{2} \cdot x_{3} \cdot x_{4}\right)
$$

The problem occurs due to the conditional probability terms which occur when we expand Equation 8.

$$
R=P\left(x_{1}\right) P\left(x_{2} / x_{1}\right) P\left(x_{3} / x_{1} x_{2}\right) P\left(x_{4} / x_{1} x_{2} x_{3}\right)
$$

In most electrical and lumped mechanical systems, these conditional probability terms reduce to independent probabilities since the elements are largely 
independent. In the case of strucutral systems, the opposite is true. Thus, in order to evaluate the conditional probability terms in Equation 9, we must model the conditional probabilities. This is a difficult task, and this problem essentially applies to all the cut-set and tie-set methods.

In addition to the complexities introduced by the statistical dependence, analysis of the basic structural system is difficult due to the factors given in Table 1. Thus, a different approach is utilized.

The system is decomposed into mutually exclusive events. In the case of mutually exclusive events, no conditional probability terms appear in the probability expansion, since by definition the probability of occurrence for product terms is identically zero for mutually exclusive events. (See Equation 2). In a probability sense, we are free to define our set of mutually exclusive events in any way which fits in with the physical model of the problem. Since the quantities which make the structure fail are the applied loads, it makes sense to define our set of mutually exclusive events in terms of the applied loads. If we assume that a hypothetical system is subject to three loads L1, L2, L3, then we can define the structural reliability as given below, and expand the reliability as given in Equation 10. 


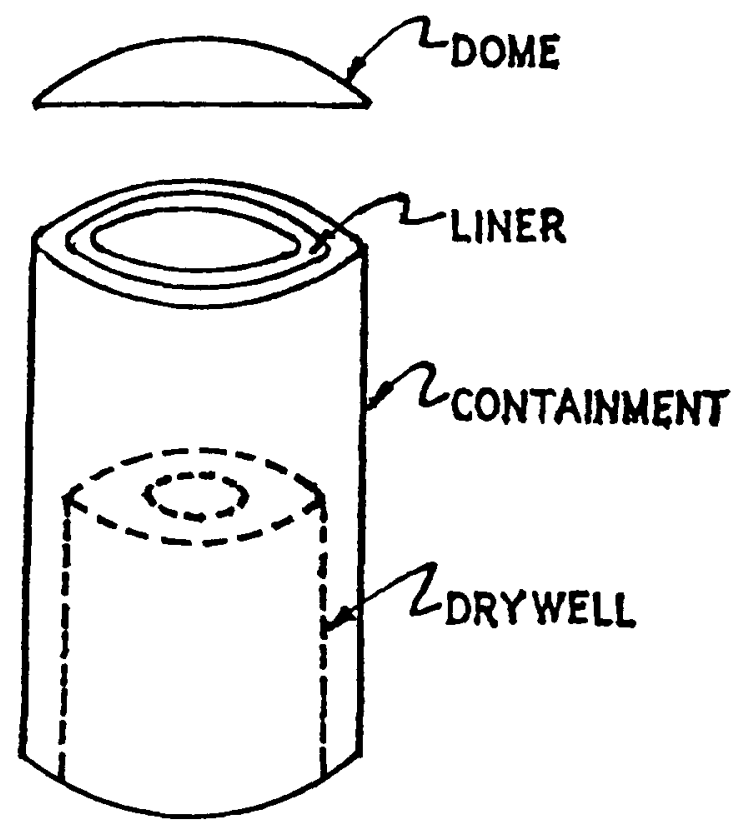

Figure 1. Typical Structural System Model

Table 1

COMPLEXITIES IN COMPUTING STRUCTURAL SYSTEM RELIABILITY 1. SEVERAL COMPONENTS IN STRUCTURE: CONTAINMENT,LINER, DOME, ETC.

2. SEVERAL POSSIBLE FAILURE MODES FOR EACH COMPONENT: COMPRESSION, TENSION, SHEAR, ETC

3. MULTIPLE APPLIED LOADS: EARTHQUAKE,SRV,PRESSURE,ETC.

4. RESPONSE COMPUTATIONS GENERALLY REQUIRE A FINITE ELEMENT COMPUTER PROGRAM.

5. NONHOMOGENEOUS RESISTANCE DUE TO COMPOSITE MATERIAL'S, EG. REINFORCED CONCRETE. 


\section{Definition of Reliability}

Structural system reliability is the probability that the structure does not fail, for a given period of time when constructed under applicable code specifications under given conditions of loading.

If loads are stochastic in nature then the reliability (based upon three simultaneous loads, $L_{1}, L_{2}, L_{3}$ ) is given by:

$$
\begin{aligned}
1-R= & P\left(L_{1} \text { OCCURS }\right) * P\left(F A I L U R E / L_{1}\right) \\
& +P\left(L_{2} \text { OCCURS }\right) * P\left(F A I L U R E / L_{2}\right) \\
& +P\left(L_{3} \text { OCCURS }\right) * P\left(F A I L U R E / L_{3}\right) \\
& +P\left(L_{1} L_{2} \text { OCCURS }\right) * P\left(F A I L U R E / L_{1} L_{2}\right) \\
& +P\left(L_{1} L_{3} \text { OCCURS }\right) * P\left(F A I L U R E / L_{1} L_{3}\right) \\
& +P\left(L_{2} L_{3} \text { OCCURS }\right) * P\left(F A I L U R E / L_{2} L_{3}\right) \\
& +P\left(L_{1} L_{2} L_{3} \text { OCCURS }\right) * P\left(F A I L U R E / L_{1} L_{2} L_{3}\right)
\end{aligned}
$$

The expansion given in Equation 10 involves 7 different probability of load occurrence terms, $P(L 1$ OCCURS), etc. These probabilities must be modeled based upon a study of the occurrence frequency statistics for each of the loads. In the case of joint occurrence of the loads, in some physical cases these will be zero. For example suppose we are talking about pressure loads, snow loads, and hurricane loads. The snow season and the hurricane season do not correspond, thus it is impossible for these two loads to occur simultaneously in most locations. In certain cases such considerations will el iminate terms in the expansion given in Equation 10.

The second set of 7 terms in Equation 10 represent the failure probabilities for various combinations of applied loads. Each of these probabilities must be calculated by combining the probabilistic methods of Appendix $\mathrm{H}$ and $\mathrm{I}$ with finite element analysis techniques as shown in the flow chart of Figure 2. 


\section{REFERENCES}

1. Zendehrovh, S. and Shinozuka, M., "A Structural and Equipment Design Methodology for Nuclear Power Plants, Technical Report No. 8, Columbia University, Department of Civil Engineering and Engineering Mechanics, April 1976.

2. Vesely. W. E., "Reliability Qualification Techniques Used in the Rasmussen Study", published in Reliability and Fault Tree Analysis, SIAM, Philadelphia, 1975, pp. 775-803.

3. Shooman, M. L., "Probabilistic Reliability and Engineerina Approach", McGraw-Hill Book Co.. New York, 1968.

4. Barlow, R. E., et al., editors, "Reliability and Fault Tree Analysis Theoretical and Applied Aspects of System Reliability and Safety Assessment", Society for Industrial and Applied Math, Philadelphia, 1975, pp.1-128. 
Appendix D

Second Order Higher Moment Approximation

D-1 


\section{APPENDIX D}

\section{Second Order Higher Moment Approximation}

Let $Y$ be a function of the random variables $x_{1}, x_{2}, \ldots, x_{n}$ :

$$
r=g\left(x_{1}, x_{2}, \ldots, x_{n}\right)
$$

Assume that the function $g\left(x_{1}, x_{2}, \ldots, x_{n}\right)$ can be expanded into a multivariate Taylor series about the mean values $\left(m_{1}, m_{2}, \ldots, m_{n}\right)$ of $x_{1}, x_{2}$, $\ldots . ., x_{n}$.

$$
\begin{aligned}
r & =g\left(m_{1}, m_{2}, \ldots ., m_{n}\right)+\sum_{i=1}^{n} \frac{\partial g}{\partial x_{i}}\left(x_{i}-m_{i}\right) \\
& +\frac{2}{2} \sum_{i=1}^{n} \sum_{j=1}^{n} \frac{\partial^{2} g}{\partial x_{i} \partial x_{j}}\left(x_{i}-m_{i}\right)\left(x_{j}-m_{j}\right)+\cdots
\end{aligned}
$$

where all the derivatives are evaluated at $x_{i}=m_{i}(i=1,2, \ldots, n)$.

Taking the expected value of Eq. 2 and retaining the terms up to the second order, we obtain the mean value $E[Y]$ of $Y$ :

$$
E[Y]=g\left(m_{1}, m_{2}, \ldots, m_{n}\right)+\frac{2}{2} \sum_{j=1}^{n} \sum_{j=1}^{n} \frac{\partial^{2} g}{\partial x_{j} \partial x_{j}} \operatorname{cov}\left(x_{f}, x_{j}\right)
$$

where

$$
\operatorname{cov}\left(x_{i}, x_{j}\right)=E\left[\left(x_{i}-m_{i}\right)\left(x_{j}-m_{j}\right)\right]
$$

If $x_{f}$ and $x_{j}$ are uncorrelated, Eq. 3 becomes

$$
E[Y]=g\left(m_{1}, m_{2}, \ldots, m_{n}\right)+k_{i} \sum_{i=1}^{n} \frac{\partial^{2} g}{\partial x_{i}^{2}} \operatorname{Var}\left(X_{i}\right)
$$


vihere

$$
\operatorname{Var}\left(x_{i}\right)=E\left[\left(x_{i}-m_{i}\right)^{2}\right]
$$

Taking the expected value of the square of Eq. 2, subtracting the square of Eq. 3 and retaining all the terms up to the third order, the variance $\operatorname{Var}(Y)$ of $Y$ is obtained as follows:

$$
\begin{aligned}
\operatorname{Var}(Y) & =\sum_{i=1}^{n} \sum_{j=1}^{n}\left(\frac{\partial g}{\partial x_{i}}\right)\left(\frac{\partial g}{\partial x_{j}}\right) \operatorname{cov}\left(x_{i}, x_{j}\right) \\
& +\sum_{i=1}^{n} \sum_{j=1}^{n} \sum_{k=1}^{n}\left(\frac{\partial g}{\partial x_{i}}\right)\left(\frac{\partial^{2} g}{\partial x_{j} \partial x_{k}}\right) E\left[\left(x_{i}-m_{i}\right)\left(x_{j}-m_{j}\right)\left(x_{k}-m_{k}\right)\right]
\end{aligned}
$$

If $X_{i}$ and $X_{j}$ are uncorrelated, then $\operatorname{Var}(Y)$ becomes

$$
\operatorname{Var}(y)=\sum_{i=1}^{n}\left(\frac{\partial g}{\partial x_{i}}\right)^{2} \operatorname{Var}\left(x_{i}\right)+\sum_{i=1}^{n}\left(\frac{\partial g}{\partial x_{i}}\right)\left(\frac{\partial^{2} g}{\partial x_{i}^{2}}\right) \mu_{3}
$$

where

$$
\mu_{3}=E\left[\left(x_{1}-m_{1}\right)^{3}\right]
$$

The variance of $Y$ in Eq. 6 utilizes the moments of the $X$ 's up to the third order, not to the fourth order as mentioned in the main text. In fact, this represents an approximation for the variance exactly one order higher (in terms of the moments of the $X ' s$ ) than the approximation resulting from the FOSM. 
If the Tavlor expansion of the function $g\left(x_{1}, x_{2}, \ldots, x_{n}\right)$ about an arbitrary point (say $x_{1}{ }^{*}, x_{2}{ }^{*}, \ldots . x_{n}^{*}$ ) is interested, then the Taylor expansion becomes as follows:

$$
\begin{aligned}
Y & =g\left(x_{1}^{*}, x_{2}^{*}, \ldots ., x_{n}^{*}\right)+\sum_{i=1}^{n}\left(\frac{\partial g}{\partial x_{j}}\right) \star\left(x_{i}-x_{i}^{*}\right) \\
& +\frac{1}{2} \sum_{i=1}^{n} \sum_{j=1}^{n}\left(\frac{\partial^{2} g}{\partial x_{i} \partial x_{j}}\right) *\left(x_{i}-x_{i}^{*}\right)\left(x_{j}-x_{j}^{*}\right)+\ldots
\end{aligned}
$$

where all the derivatives are evaluated at $x_{i}=x_{i}{ }^{*}(i=1,2, \ldots, n)$.

Following the similar procedure, the mean value $E[Y]$ of $Y$ are approximated as follows:

$$
\begin{aligned}
& E[Y]=g\left(x_{1}{ }^{*}, x_{2}{ }^{*}, \ldots, x_{n}{ }^{*}\right)+\sum_{i=1}^{n}\left(\frac{\partial g}{\partial X_{i}}\right) * E\left[\left(X_{i}-x_{i}^{*}\right)\right] \\
& +\frac{1}{2} \sum_{i=1}^{n} \sum_{j=1}^{n}\left(\frac{\partial^{2} g}{\partial x_{i} \partial x_{j}}\right) * E\left[\left(x_{i}-x_{i}^{*}\right)\left(x_{j}-x_{j}^{*}\right)\right] \\
& \operatorname{Van}(Y)=\sum_{i=1}^{n} \sum_{j=1}^{n}\left(\frac{\partial g}{\partial X_{i}}\right) *\left(\frac{\partial g}{\partial X_{j}}\right) *\left\{E\left[\left(x_{j}-x_{i}^{*}\right)\left(x_{j}-x_{j}^{*}\right)\right]-E\left[\left(x_{i}-x_{i}^{*}\right)\right]\right. \\
& \left.E\left[\left(x_{j}-x_{j}^{*}\right)\right]\right\}+\sum_{i=1}^{n} \sum_{j=1}^{n} \sum_{k=1}^{n}\left(\frac{\partial g}{\partial x_{j}}\right) *\left(\frac{\partial^{2} g}{\partial x_{j} \partial x_{k}}\right) *\left\{E\left[\left(x_{i}-x_{j}^{*}\right)\left(x_{j}-x_{j}^{*}\right)\left(x_{k}-x_{k}^{*}\right)\right]\right. \\
& \left.-E\left[\left(x_{i}-x_{i}^{*}\right)\right] E\left[\left(x_{j}-x_{j}^{*}\right)\left(x_{k}-x_{k}^{*}\right)\right]\right\}
\end{aligned}
$$

It is noted that the moments of $Y$ about the point $\left(x_{1}{ }^{*}, x_{2}{ }^{*} \ldots, x_{n}{ }^{*}\right)$, i.e., $E\left[\left(x_{i}-x_{i}^{*}\right)\right], E\left[\left(x_{i}-x_{i}^{*}\right)\left(x_{j}-x_{j}^{*}\right)\right]$, etc., can be evaluated easily from samples. 
Appendix E

General Forms of Probability Density Functions 


\section{General Forms of Probability Density Functions}

This appendix describes the Gram-Charlier series of Types $A$ and $C$ and the asymptotic series that may be used for the general representation of probability density functions. For details, see Reference 1.

\section{(a) Gram-Charlier Series of Type A}

The probability density function $f(x)$ for the standardized variable of an original random variable $\xi$ can be expanded into the following Gram-Charlier series of Type A under certain general conditions. 2

$$
f(x)=\phi(x)+\frac{C_{3}}{3 !} \phi^{(3)}(x)+\frac{C_{4}}{4 !} \phi^{(4)}(x)+\frac{C_{5}}{5 !} \phi^{(5)}(x)+\cdots
$$

where

$$
\phi(x)=\frac{1}{\sqrt{2 \pi}} e^{-x^{2} / 2}
$$

and

$$
\phi^{(v)}(x)=d^{v}(x) / d x^{v}
$$

The coefficients $C_{v}$ can be determined by using the orthogonality between $\phi^{(v)}(x)$ and the Hermite polynomials $H_{v}(x)$, shown as follows:

$$
C_{3}=-\mu_{3} / \sigma^{3}, \quad C_{4}=\mu_{4} / \sigma^{4}-3, \quad C_{5}=\mu_{5} / \sigma^{5}+10_{3} / \sigma^{3}, \cdots
$$

where $\mu_{v}$ and $\sigma$ are the $v$-th central moment and standard deviation, respective$1 y$, of the original random variable $\boldsymbol{\xi}$.

In reality, however, the probability density function $f(x)$ must be ap- 
proximated by truncating the series in Eq. 1 after a finite number of terms and replacing $\mu_{v}$ and $\sigma$ by corresponding sample moments and sample standard deviation, respectively. It is noted that the density function approximated by such a truncated series with the coefficients evaluated on the basis of sample moments may produce negative densities for large values of $|x|$. If needed, the density function $f(\xi)$ of $\xi$ can be obtained from $f(x)$ by means of a linear transformation.

(b) Gram-Charlier Frequency Function of Type C

The probability density function $f(x)$ can also be expressed in the form of a Gram-Charlier frequency function of type $C$ which is always positive.

$$
f(x)=\exp \left[\sum_{0}^{\infty} \gamma_{\nu}{ }_{v}(x)\right]
$$

where $H_{v}(x)$ are Hermite polynomials. Aga in, with the aid of the orthogonality between $\phi^{(v)}(x)$ and $H_{v}(x)$, the coefficients $\gamma_{v}$ are determined as follows:

$$
\gamma_{v}=\frac{(-1)^{\nu}}{v !} \int_{-\infty}^{\infty}\{\ln f(x)\} \phi^{(v)}(x) d x
$$

Numerically, $\gamma_{v}$ may be evaluated by approximating the integral above with summation and replacing $f(x)$ by the empirical frequencies from the sample.

$$
\gamma_{v}=\frac{(-1)^{\nu}}{v !} \sum_{i}\left(\ln f_{i}\right\}_{\phi}^{(v)}\left(x_{i}\right) \Delta x
$$

where $f_{f}$ are the observed relative frequencies in the $i$-th interval $\left(x_{f}-\Delta x / 2\right.$, $\left.x_{i}+\Delta x / 2\right)$.

The probability density function $f(x)$ is usually approximated by taking the first few terms, say up to $n$, of the series in Eq. 5. Even then, the ap- 
proximated density is alviays positive. However, since the sum $\sum_{0}^{n} r_{v} H_{v}(x)$ is essentially a polynomial, it may not behave well for large values of $|x|$.

(c) The Asymptotic Series

The probability density function $f(x)$ can be evaluated from its characteristic function $\bar{f}(\omega)$

$$
f(x)=\frac{1}{2 \pi} \int_{-\infty}^{\infty} \bar{f}(\omega) e^{-i \omega x} d \omega
$$

where

$$
\bar{f}(\omega)=\int_{-\infty}^{\infty} f(x) e^{i \omega x} d x
$$

It can be shown that $\bar{f}(w)$ in Eq. 9 has the following al ternate series expression:

$$
\bar{f}(\omega)=e^{-k \omega^{2}}\left(1+\sum_{k=3}^{\infty} A_{k} i^{k} \omega^{k}\right)
$$

where $f$ is an imaginary unit and

$$
A_{3}=\frac{\mu_{3}}{3 ! \sigma^{3}}, \quad A_{4}=\frac{1}{4 !}\left(\frac{\mu_{4}}{\sigma^{4}}-3\right), \quad A_{5}=\frac{1}{5 !}\left(\frac{\mu_{5}}{\sigma^{5}}-10 \frac{\mu_{3}}{\sigma^{3}}\right)
$$

Substituting Eq. 10 into Eq. 8 and carrying out the integration by parts repeatedly, the asymptotic expansion for $f(x)$ is obtained as follows:

$$
f(x) \sim \phi(x)\left(1+\frac{c_{3}^{\prime}}{x^{3}}+\frac{c_{4}^{\prime}}{x^{4}}+\frac{c_{5}^{\prime}}{x^{5}}+\cdots \cdot\right)
$$

where 


$$
\begin{aligned}
& c_{3}^{\prime}=\mu_{3} / \sigma^{3}=-c_{3} \\
& c_{4}^{\prime}=\mu_{4} / \sigma^{4}+9 \mu_{3} / \sigma^{3}-3=c_{4}-9 c_{3} \\
& c_{5}^{\prime}=\mu_{5} / \sigma^{5}+14 \mu_{4} / \sigma^{4}+35 \mu_{3} / \sigma^{3}-42=-c_{5}+14 C_{4}-45 C_{3}
\end{aligned}
$$

Replacing $\mu_{v}$ and $\sigma$ by the sample moments and sample standard deviation and truncating the series in Eq. 12 after a few terms, an asymptotic approximation of $f(x)$ is obtained. For such an asymptotic approximation of $f(x)$, one can always find a value $x_{0}$ so that the approximation is positive for $|x|$ $>x_{0}$ and monotonically decreasing as $|x|+\infty$.

\section{(d) Example}

In Fig. 1, a histogram is constructed out of a total sample of 449 measurements of yield strength of steel $\xi^{3}$. Frequencies based on the Gram-Charlier series of type $A$, the Gram-Charlier frequency function of type $C$ and the asymptotic expansion obtained from this sample are drawn. Both types $A$ and C fit the data quite well in the central portion of the distribution. However, type A produces negative values (Table 1 ; too small to observe on the diagram) for large and small vālues of $\xi$ whereas the curve for type $C$ behaves wildly as seen directly on the diagram for small and large values of 5 . On the other hand, the asymptotic expansion gives rise to well behaved curves that remain positive and decreasing as $|\xi|$ increases (Table 1 ). The number of terms taken in the series are indicated by the corresponding frequency curves.

(e) Conclusion

In order to establish analytical forms of the density functions of the resistance of structures and of the applied load from samples of finite size 
to be used in the structural reliability analysis, the methods of series expansion by means of the asymptotic series and the Gram-Charlier series of types $A$ and $C$ are reviewed with a numerical example. It appears reasonable to state that the Gram-Charlier series of both types produce a desirable result in the central portion of the distribution and that the simultaneous use of the Gram-Charlier series of type $A$ to cover the central portion and the asymptotic expansion to cover the tail end portions will be successful in many practical cases.

\section{REFERENCES}

1. Shinozuka, M. and Nishimura, A., "On General Representation of a Density Function," Annals of Reliability and Ma intainability, Fourth Annual Reliability and Maintainability Conference, Volume 4 - Practical Techniques and Application, July 28-30, 1965, Pp. 897-903.

2. Cramer, H., Mathematical Methods of Statistics, Princeton University Press, 1958 , p. 222 .

3. Dieter, G.E., Jr., Mechanical Metallurgy, McGraw-Hill, New York, p. 421. 


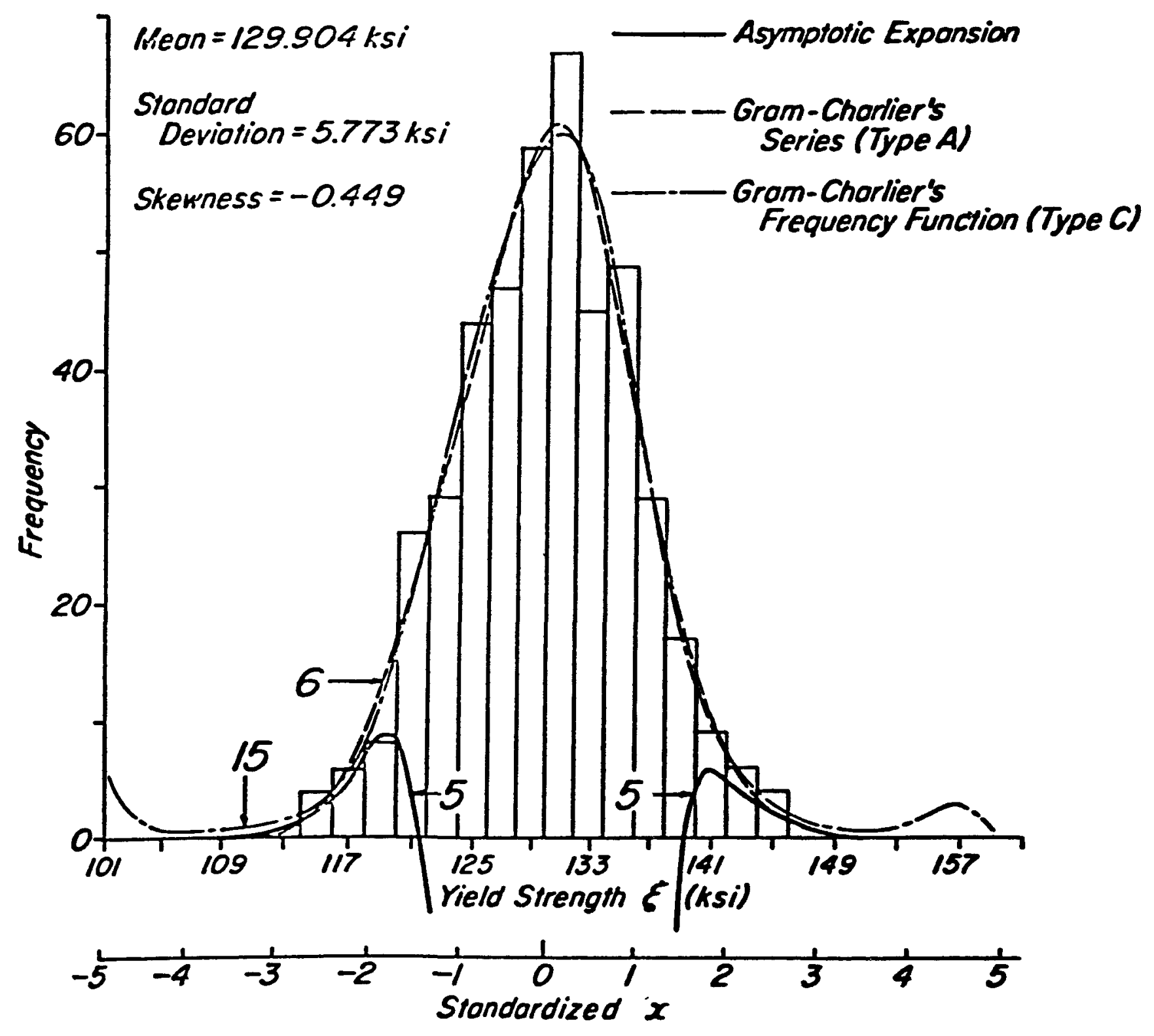

18. 1. Frequencles fltted to dietribution of gleld etrength
of eteel. 


\section{Table 1}

Frequencles estimated for the distribution of Jield strength of steel

\begin{tabular}{|c|c|c|}
\hline$\xi(k 81)$ & $\begin{array}{l}\text { Sram-Charlier } \\
\text { serles of type } A\end{array}$ & $\begin{array}{l}\text { Asymptotic } \\
\text { expansion }\end{array}$ \\
\hline $\begin{array}{l}106-108 \\
108-110 \\
110-112 \\
112-114 \\
114-116\end{array}$ & $\begin{array}{l}-2.356 \times 10^{-2} \\
-1.896 \times 1 \\
1.207 \times 10^{-1} \\
7.145 \times 1 \\
2.367\end{array}$ & $\begin{array}{l}3.031 \times 10^{-2} \\
1.078 \times 10^{-1} \\
3.410 \times n \\
9.571 \times n \\
2.369\end{array}$ \\
\hline $\begin{array}{l}146-148 \\
148-150 \\
150-152 \\
152-154 \\
154-156\end{array}$ & $\begin{array}{l}8.026 \times 10^{-1} \\
2.340 \times 1 \\
3.658 \times 10^{-2} \\
-1.200 \times 1 \\
-1.383 \times 1\end{array}$ & $\begin{array}{l}8.454 \times 10^{-1} \\
3.017 \times n \\
9.496 \times 10^{-2} \\
2.650 \times n \\
6.571 \times 10^{-3}\end{array}$ \\
\hline
\end{tabular}




\section{Appendix F}

\section{Bounded Tail Probabilities Using}

Inequalities of the Chebycheff Type 


\section{Introduction}

Much of the methodology presently being used to perform probabilistic design of structures involves use of a reliability index (safety index), B. The problem arises as to how to convert this reliability index into a probability of failure. Direct computation of the distribution of $\beta$ involves a complex (and generally intractable) problem in transformation of random variables. Thus, we seek distribution free methods to obtain approximations or bounds on the probabilities in the tail of the distribution of $B$.

\section{Approach}

A family of probability inequalities of the Chebycheff type have been developed over the years, (see References 1,2). In general these inequalities provide an upper bound on the probability that the random variable deviates from the mean by a specified amount.

For example, one of the simplest bounds of this class, the Chebycheff bound, shows that the probability that the random variable, $x$, deviates from the mean, $\mu$, by more than $\lambda$ standard deviations, $\lambda \sigma$, is less than or equal to $1 / \lambda^{2}$. Thus, the deviation of more than two standard deviations from the mean is bounded by 0.25 . The Chebycheff inequality does not require the imposition of any restrictions on the random variable. Consequently, the bound is not very sharp. For example, if we knew that the random variable had a normal distribution then the area under one tail of the distribution to the right of $2 \sigma$ is found from a table of normal probabilities to be 0.0228 . Thus, the bound is an order of magnitude above the actual value.

More advanced inequalities of this type place some restrictions on the random variable or its moments, and are able to achieve a sharper 
bound. Thus, in practice we try to compile what we know about the characteristics of the random variable, $x$, try all applicable limit theorems, and adopt the one with the sharpest bound which applies.

3. Comparison of Bounds

In order to explore the use of these bounds, three different inequalities are studied, Chebycheff, Gauss and Bernstein. The inequalities and the associated restrictions on $x$ are given in Table $\dot{1}$.

In each case the inequality is two sided, i.e., it gives the probability that the deviation above and below the mean is greater than $\lambda \sigma$. In our physical problem, we are only interested in the probability that the deviation is above $\lambda \sigma$. Thus, if we can show that the distribution of $X$ is symmetrical, we can reduce each bound by a factor of two.

In order to compare the various bounds we present the results obtained by applying the three inequalities in Table 2. In each case we have assumed that $X$ is symmetrically distributed and have decreased the bound by a factor of two. The values chosen for $\lambda$ vary from 2 to 4 , since $\lambda$ is essentially the reliability index $\beta$, which normally falls within this range. To provide a baseline for evaluating the sharpness of the bounds, the tail area under a normal distribution is also given.

4. Results and Conclusions

An examination of Table 2 shows that the Bernstein bound and the Gauss bound are about equally sharp for $\lambda=2$ and 2.5. In this range these bounds overestimate the normal distribution tail area by a factor of 3-5. For $\lambda$ above 3, the Bernstein bound is superior and it overestimates the normal tail area by a factor of 4-5. 
It should be noted that a bound which is off by a factor of 5 (one-half an order of magnitude) is still of great use in many cases. Suppose we wish to know if the probability of failure is $10^{-4}$ or $10^{-6}$, then such a bound is sharp enough. More work must be done to show whether the distribution is symmetrical, whether the restrictions on the moments for the Bernstein bound are indeed satisfied, if there are other bounds which are sharper or have more easily satisfied restrictions, and to estimate the sharpness of the bounds for other than Normal Distributions. 


\section{REFERENCES}

1. I. Richard Savage, "Probability Inequalities of the Chebycheff Type", Journal of Research, National Bureau of Standards, Mathematics and Mathematical Physics, Vol. 65B, No. 3, July September, 1961.

2. H. J. Goodwin, "On Generalizations of Chebycheff's Inequality", Journal of American Statistical Association, No. 50, 1955, pp. 923-945. 
Table 1

Inequalities Studied. (See Reference 1).

2. Bienayme-Tchebycheff

$$
\begin{aligned}
& P(|X-\mu| \geq \lambda \sigma) \leq 1 / \lambda^{2} \\
& P(X \geq \lambda \sigma+u \text { or } X \leq \mu-\lambda \sigma) \leq 1 / \lambda^{2} \\
& P(|X-\mu| \geq \lambda) \leq \sigma^{2} / \lambda^{2}
\end{aligned}
$$

Random variable: $X$ Restrictions: None

Dimension: One

Moments: $\quad \sigma^{2}=E(X-\mu)^{2}$

9. Gauss (Camp-Meide11)

13b. Bernstein

$$
P(|X-\mu|>\sigma \lambda) \leq e^{-\lambda^{2} / 2}
$$

$P\left(\left|X-\mu_{0}\right| \geq \lambda \tau\right) \leq\left\{\begin{array}{l}1-\frac{\lambda}{\sqrt{3}} \text { for } \lambda \leq \frac{2}{\sqrt{3}}=1.1547 \\ \frac{4}{9 \lambda^{2}} \text { for } \lambda \geq \frac{2}{\sqrt{3}}\end{array}\right.$

Random variable: $X=\Sigma X_{i}$

Restrictions: $x_{i}, x_{j}$ are independent

$(i \neq j) ; x_{j}$ is symmetrical about $\mu_{j}$; i.e., $E\left(x_{i}-\mu_{i}\right) 2 r+1=0 \quad(r=0)$,

Dimension: One

Moments: $\mu_{i}=E X_{i}, \mu=\Sigma \mu_{i}$

$P(|X-\mu| \geq \lambda \sigma) \leq \frac{4}{9} \cdot \frac{1+s^{2}}{(\lambda-s)^{2}}$ if $\lambda>s$

$$
E\left(x_{i}-\mu_{i}\right)^{2 r} \leq\left(\frac{\sigma_{i}^{2}}{2}\right)^{r} \frac{(2 r) !}{r !}(r \text { an integer }
$$

$$
\sigma_{i}^{2}=E\left(x_{i}-\mu_{i}\right)^{2}, \sigma^{2}=\Sigma \sigma_{i}^{2}
$$

Random variable: $X$

Restrictions: $X$ has a density function with one mode, $\mu_{0}$

Dimension: One

Moments: $\mu=E X, \sigma^{2}=E(X-\mu)^{2}, \tau^{2}=\sigma^{2}+\left(\mu+\mu_{0}\right)^{2}$

$$
s=\left|\frac{\mu-\mu_{0}}{\sigma}\right|
$$

Note: The case where the mean $=$ the mode, $(s=0)$, was studied. 
Table 2

A Comparison of Chebycheff, Gauss, and Bernstein Bounds

\begin{tabular}{lllll}
\hline$\lambda$ & Normal & Chebycheff & Gauss & Bernstein \\
\hline 2.0 & 0.023 & 0.13 & 0.056 & 0.068 \\
2.5 & 0.0062 & 0.08 & 0.036 & 0.022 \\
3.0 & 0.0013 & 0.056 & 0.024 & 0.0056 \\
3.5 & 0.00018 & 0.041 & 0.018 & 0.0011 \\
4.0 & 0.000033 & 0.031 & 0.014 & 0.00017 \\
\hline
\end{tabular}


$\because$ 
FIGURE 2

START

$\downarrow$

METHODOLOGY

FLOW CHART

FOR

STRUCTURAL

SYSTEMS

RELIABILITY

COMPUTATION

READ $\mu$ AND $\sigma$

FOR RESISTANCES IN EACH FAILURE

MODE OF ALL COMPONENTS

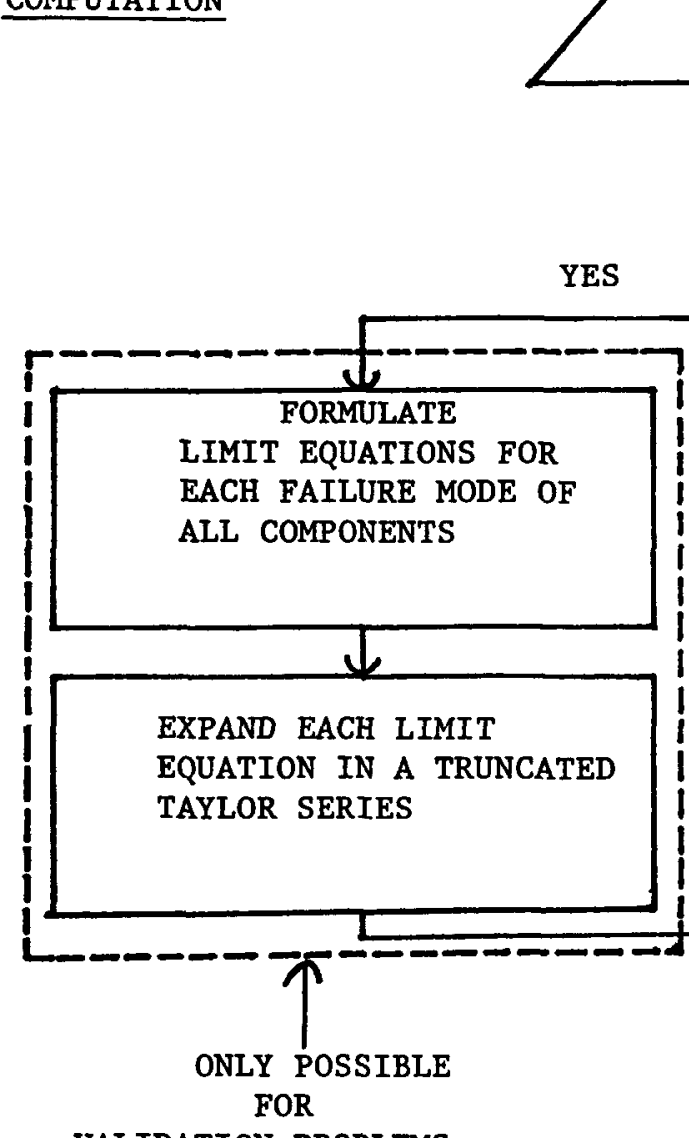

VALIDATION PROBLEMS

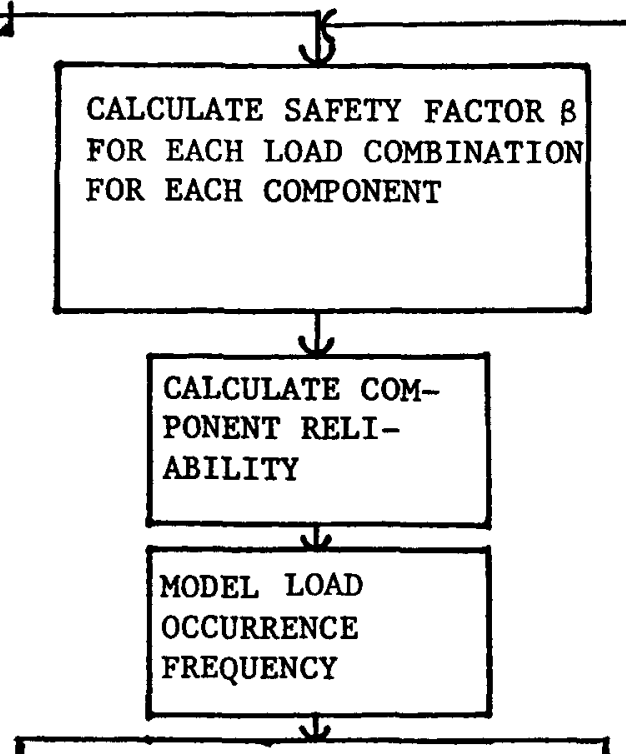

NO

FORM SOLUTION

POSSIBLE FOR LOAD

EFFECTS

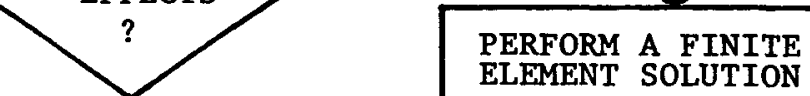

ELEMENT SOLUTION

CALCULATE SYSTEM

RELIABILITY BASED UPON

ABOVE SOLUTIONS

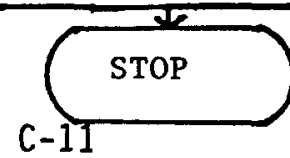


Appendix G

Comments on the Application of Upcrossing

Methods to Load Combinations 


\section{Level Crossing Method}

The method using up-crossing rate for computing the probability of exceeding a specified load level has appeared in the recent literature. ${ }^{[1,2]}$ The method is summarized as follows:

Let the random process $Z(t)$ be the sum of two stationary independent random processes $Y_{1}(t)$ and $Y_{2}(t)$.

$$
Z(t)=Y_{1}(t)+Y_{2}(t)
$$

where $Y_{j}(t)$ is the $i$-th response process with duration $T_{i}$ and

$$
Y_{i}(t)=A_{i} X_{i}(t)
$$

in which

$$
\begin{aligned}
& \max \left[X_{j}(t)\right]=1 \\
& \max \left[Y_{i}(t)\right]=A_{i}
\end{aligned}
$$

Assume that the number of upcrossings of the combined process $z(t)$ to exceed level a in time from 0 to $T\left(T=T_{1}+T_{2}\right)$ is governed by a Poisson distribution, the probability of no crossina $F(a)$ is known as follows:

$$
F(a)=e^{-\lambda T}
$$

in which $\lambda$ is the expected occurrance rate and here it is equal to the mean upcrossing rate $v_{a}$ of $Z(t)$. Therefore Equation 3 can be rewritten as

$$
F(a)=e^{-\nu} a^{T}
$$

If $v_{d} T \ll 1$ and $e^{-v_{a}}{ }^{\top}$ is expanded into power series and retained only the first two terms. Equation 4 becomes

$$
F(a) \simeq 1-\nu_{a}^{\top} \text { or } 1-F(a) \simeq \nu_{a}^{\top}
$$


It can be proved that the probability of one or more crossings is bounded by $\nu_{a} T^{[2]}$ The proof is as follows. The expected number of upcrossings of level a in time from 0 to $T$ is

$$
v_{\mathrm{a}} \mathrm{T}=\sum_{j=1}^{\infty} j \times \operatorname{Pr} \text { (exactiy } \mathrm{j} \text { crossings) }
$$

and the probability of one or more crossings is given by

$$
1-F(a)=\sum_{j=1}^{\infty} \operatorname{Pr} \text { (exactly } j \text { crossings) }
$$

Since every term in Equation 6 is an integer multiple of the corresponding term in Equation 7, the Equation 7 is bounded by Equation 6 . That is

$$
1-F(a) \leq v_{a} T
$$

Corne11 ${ }^{[2]}$ assumes that if $\nu_{a}{ }^{\top} \ll 1$ then the bound in Equation 8 is sharp and can be used as an approximation. This assumption leads to the same formula as shown in Equation 5 which is derived under Poisson assumption with the condition that $v a T<1$. Cornel1 ${ }^{[2]}$ also states that the assumption of Poisson arrivals of the upcrossings may give the probability of one or more crossing an even closer approximation, especially at lower values of a. 2. Investigation of the Validity of the Poisson Assumption

The Poisson distribution is assumed for deriving Equation 4. The assumbtion is tested twice using the data from Reference 3. From that report (Reference 3 ), we select two sets of earthquake responses for $Y_{1}(t)$ (Sets 63, 14, main steam 1I OBE, shroud support OBE) and two sets of SRV responses for $Y_{2}(t)$ (Sets 45,32 , main steam 11 SRV, shroud support ASRV). Pair 1 represents the random combinaion of sets 63 and 45 and pair 2 represents the random combination of sets 14 and 32 . 
The responses are digitized and are made stationary by following Cornell's procedure. [1] Then we combine the responses by uniformly shifting one with respect to the other with $k \Delta t$ each time where $k$ is an integer and $\Delta t$ is the digitizing interval. For the combined response $Z(t)$, the number of crossing of level a from time 0 to $T$ is calculated. The above procedure is repeated for eight different levels, some above and some below SRSS value.

By applying the Pearson $x^{2}$ test to the number of crossings, we can study the qoodness of fit for the Poisson assumption. The $\chi^{2}$-test results are summarized in Tables 1 and 2 and the empirical probability densities for the number of crossings are given in Tables 3 and 4 .

By inspecting the results, we have the following observations. In Pair 1, if the level of crossing a is above SRSS, the crossing phenomenon is a rare event (see Table 3, zero-crossing probability), so the Poisson assumption naturally holds. If the level a is at or below SRSS, the $x^{2}$ tests reject the Poisson assumption except at a level near SRSS. In Pair 2, all the indicated levels except the last level at 132, the crossing phenomenon also is a rare event (see Table 4, zero-crossing probability), so the Poisson assumption holds naturally. The $x^{2}$ test strongly rejects the Poisson assumption at level 132, even through the dominant number of crossing is shifted slightly (from zero-crossing to one-crossing).

The actual probabilities of no-crossing and the corresponding value under Poisson assumption with $\nu_{a}$ estimated from the data are shown in Table 5. We can see that the probability of no-crossing is considerably overestimated if the Poisson assumption is rejected by the $x^{2}$ tests.

3. Validity of the Approximate Formula for $v_{a}$

To evaluate Equation 4. Cornell proposed the following approximate formula for computing $\nu_{a}$ (see Reference 1. Equation A4).

$$
\nu_{a} \simeq \int_{-\infty}^{\infty} v_{Y_{1}}(y) f_{Y_{2}}(a-y) d y+\int_{-\infty}^{\infty} v_{Y_{2}}(y) f_{Y_{1}}(a-y) d y
$$


In order to study the validity of the approximation in Equation 9 the responses $Y_{1}(t)$ and $Y_{2}(t)$ defined in Figure 1 are considered. Note that $Y_{1}(t)$ and $Y_{2}(t)$ are the same in this example. By following the Cornell procedure ${ }^{[1]}$, the responses $Y_{1}(t)$ and $Y_{2}(t)$ are modified to be stationary with period $T=T_{1}+T_{2}$. The first term in Equation 9 can be calculated as follows:

$$
\int_{-\infty}^{\infty} v_{Y_{1}}(y) f_{Y_{2}}(a-y) d y=\int_{-b}^{b} \frac{N}{4 N b} f_{Y_{2}}(a-y) d y=\frac{1}{4 b}\left[F_{Y_{2}}(a+b)-F_{Y_{2}}(a-b)\right]
$$

If we set $a=\sqrt{2 b}$ (SRSS value), then $a+b=(\sqrt{2}+1) b$ and $a-b=(\sqrt{2}-1) b$. It is easy to see that

$$
\begin{aligned}
& F_{Y_{2}}(a+b)=1 \\
& F_{Y_{2}}(a-b)=\frac{2 N b}{4 N b}+\frac{N(\sqrt{2}-1) b}{4 N b}+\frac{N b}{4 N b}=\frac{\sqrt{2}+2}{4}
\end{aligned}
$$

where $N$ is number of peaks. Substituting Equations 11 and 12 into Equation 10, then Equation 10 becomes

$$
\int_{-\infty}^{\infty} \nu_{Y_{1}}(y) f_{Y_{2}}(a-y) d y=\frac{2-\sqrt{2}}{16 b}
$$

Similarly, we get

$$
\int_{-\infty}^{\infty} v_{Y_{2}}(y) \quad f_{Y_{1}}(a-y) d y=\frac{2-\sqrt{2}}{16 b}
$$

Therefore, the approximate value of $v_{a}$ (R-H-S of Equation 9) is

$$
\nu_{a}^{*} \text { (approx.) }=1.172 / 16 \mathrm{~b}
$$

On the other hand, if we randomly shift one response with respect to the other uniformly over the whole duration $T=4 \mathrm{Nb}$ as described in Reference 1, we obtain the exact value of $\nu_{a}$ (L-H-S of Equation 9) as follows: 


$$
\begin{aligned}
\nu_{a} \text { (actual) }= & \frac{1}{4 N b}\left\{\frac{b-b / \sqrt{2}}{4 N b}[N+2(1+2+\ldots+(N-1))]+\right. \\
& \frac{b / \sqrt{2}}{4 N b}[N+2(1+2+\ldots+(N-1)]\} \\
& v_{a} \text { (actual) }=1 / 16 b
\end{aligned}
$$

The relative difference is

$$
\frac{v_{a} \text { (approx.) }-v_{a} \text { (actual) }}{v_{a} \cdot \text { (actual) }}=17 \%
$$

Since the approximate value of $v_{a}$ is larger than the actual value of $v_{a}$ in this example, this offers a support that Equation 9 is a conservative approximate formula for $v_{a}$.

However, if we proceed to calculate the probability of no-crossing, $F(a)$, using Eauation 4 with approximate and exact value of $v_{a}$ in this example, we get

$$
\begin{aligned}
& {[F(a)] \text { approx. }} \\
& =e^{-0.293 N} \\
& {[F(a)] \text { actual }=e^{-0.25 N}}
\end{aligned}
$$

The relative ratio of $F(a)$ is

$$
a=\frac{[F(a)] \text { approx. }}{[F(a)] \text { actuaT }}=\frac{e^{-0.293 N}}{e^{-0.25 N}}=(0.95788)^{N}
$$

The ratio $\alpha$ is a function of the number of peaks $N$ (and therefore of $T$ ). For example, if $N=10$, then $\alpha=0.65$. That is the approximate value of $F(a)$ is only about $65 \%$ of the true value, even if the $v_{a}$ is only overestimated $17 \%$. 
4. Conclusions

The validities of the assumption of Poisson arrivals of upcrossings and the approximate formula for mean upcrossing rate $\nu_{a}$ are investigated. The Poisson assumption required to derive $F(a)$ in Equation 4 is not necessarily valid unless the process in question is first tested to see if it satisfies the Poisson assumption for the crossing level of interest. Moreover, the use of Poisson assumption without verification leads to an overestimate of the probability of no crossing (see Table 5). In engineerina application if we interpret probability of no-crossings as a measure of reliability, this overestimate is not on the safe side.

On the other hand, the use of conservative aporoximate formula for $\nu_{a}$, Equation 9, may lead to a considerable underestimate of the probabilitv of no-crossings under Poisson assumption. It is therefore suspected that the Equation 9 is too conservative to be used in calculation of the probabilities of no-crossings. 


\section{REFERENCES}

1) Corne11, C. A. et al., An Analytical Method for Determining the Probability Distribution of the Maximum of Combined Responses, Report No. 10, 1979, for N. M. Newmark Consultants, December 1979.

2) Larrabee, R. D. and Cornel1, C. A., Upcrossing Rate Solution for Load Combinations, Journal of Structural Division, ASCE, January 1979, pp. 125-132.

3) Reich, M. et al., Review of Methods and Criteria for Dynamic Combinations in Piping Systems, NUREG/CR-1330, March 1980. 
Table 1

Summary of $\chi^{2}$ test for Pair 1. Rejection level is determined for $5 \%$ significance, D.F. means degree of freedom.

\begin{tabular}{lrrcr}
\hline \multicolumn{1}{c}{ a } & \multicolumn{1}{c}{$x^{2}$} & D.F. & Rejection Level & Conclusion \\
\hline 1120 & 0.00 & 1 & 3.84 & accept \\
1058 & 0.07 & 1 & 3.84 & accept \\
997 & 5.46 & 2 & 5.99 & accept \\
936 (SRSS) & 22.78 & 3 & 7.81 & reject \\
875 & 3.80 & 3 & 7.81 & accept \\
814 & 82.91 & 3 & 7.81 & reject \\
752 & 230.61 & 3 & 7.81 & reject \\
691 & 782.37 & 3 & 7.81 & reject \\
\hline
\end{tabular}

Table 2

Summary of $x^{2}$ test for Pair 2. Rejection level is determined for $5 \%$ significance, D.F. means degree of freedom.

\begin{tabular}{lrrrr}
\hline \multicolumn{1}{c}{ a } & \multicolumn{1}{c}{$\chi^{2}$} & D.F. & Rejection Level & Conclusion \\
\hline 197 & 0.00 & 1 & 3.84 & accept \\
189 & 0.00 & 1 & 3.84 & accept \\
181 & 0.00 & 1 & 3.84 & accept \\
173 (SRSS) & 0.00 & 1 & 3.84 & accept \\
164 & 0.00 & 1 & 3.84 & accept \\
156 & 0.01 & 1 & 3.84 & accept \\
148 & 0.04 & 1 & 3.84 & accept \\
140 & 0.78 & 2 & 5.99 & accept \\
132 & 1909.66 & 3 & 7.81 & reject \\
\hline
\end{tabular}


Table 3

Empirical Probability Densitites for the Number of Crossings at Various Levels for Pair 1.

\begin{tabular}{cccccc}
\hline Level & \multicolumn{1}{c}{ (c) } & & & \\
\hline $\mathrm{a}$ & 0 & 1 & 2 & 3 & 4 \\
\hline 1058 & .950 & .050 & & & \\
936 (SRSS) & .772 & .178 & .036 & .013 & \\
814 & .128 & .497 & .300 & .075 & 0 \\
691 & .0 & .033 & .176 & .761 & .031 \\
\hline
\end{tabular}

Table 4

Empirical Probability Densities for the Number of Crossings at Various Levels for Pair 2

\begin{tabular}{lccccc}
\hline Level & \multicolumn{5}{l}{ Number of Crossings } \\
\hline 189 & 0 & 1 & 2 & 3 & 4 \\
173 (SRSS) & .999 & .001 & & & \\
156 & .982 & .018 & 0 & 0 \\
140 & .949 & .048 & .002 & 0 \\
132 & .016 & .934 & .042 & .008 \\
\hline
\end{tabular}


Table 5

Probability of no crossing, $F(a)$

\begin{tabular}{cccc}
\hline Leve 1 & Pair & {$[F(a)]$ actual $^{*}$} & {$[F(a)]$ Poisson $^{\star \star}$} \\
\hline 814 & 1 & 0.128 & 0.267 \\
691 & 1 & 0 & 0.061 \\
156 & 2 & .982 & .982 \\
140 & 2 & .949 & .949 \\
132 & 2 & .016 & .353 \\
\hline
\end{tabular}

* $[F(a)]$ actual is the probability of no crossing computed from the data.

${ }^{\star *}[F(a)]$ poisson is the probability of no crossing under Poisson assumption with $v_{a}$ estimated using the distribution of actual crossings. 
$Y_{1}(t)$ and $Y_{2}(t)$

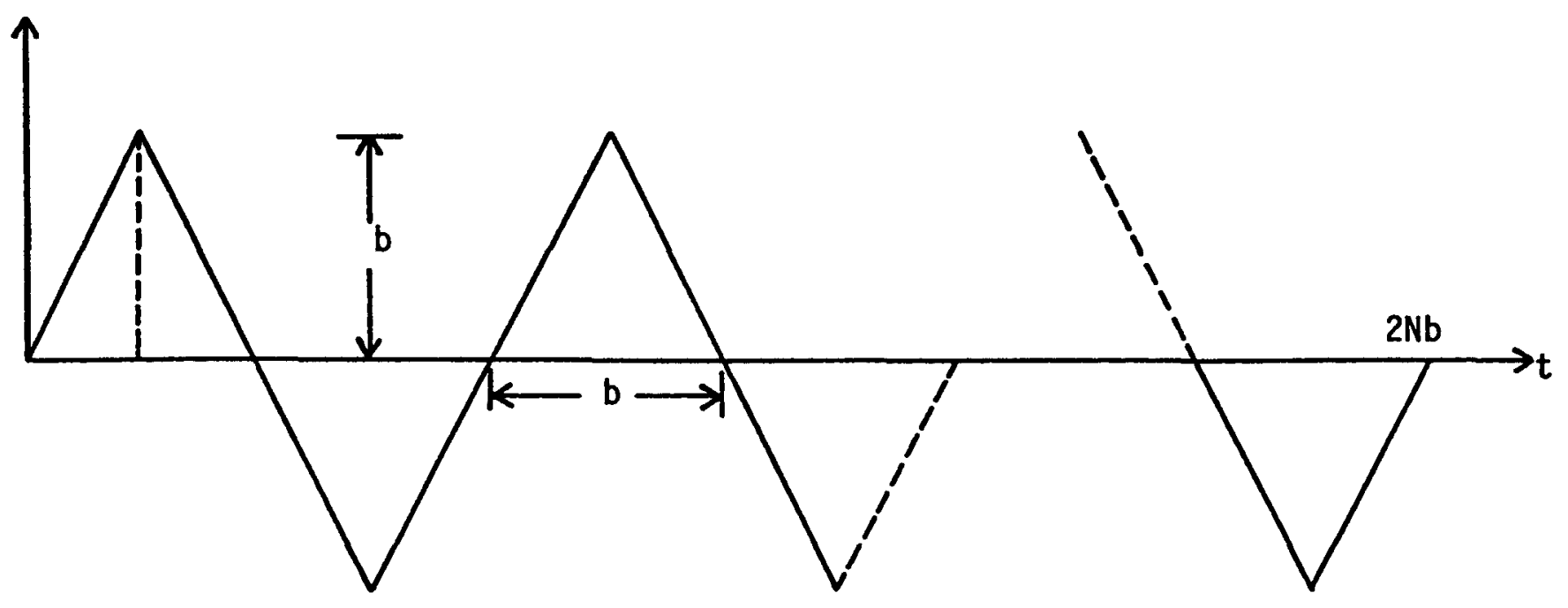

Figure 1. Periodic Triangular Responses

( $N$ is no. of cycles, $T_{1}=T_{2}=2 N b$ ) 


\section{Appendix H}

Notes on the Combinations of Random Loads I 
NOTES ON THE COMBINATIONS OF RANDOM LOADS (I)

by

\author{
M. Shinozuka \\ Renwick Professor \\ of Civil Engineering \\ Columbia University \\ New York, N.Y. 10027
}

July 1980

Prepared for

Brookhaven National Laboratory

under Grant No. BNL-517722-S

H-2 
The following notes present some of the analytical results that pertain to the problem of so-called "load combinations," and more specifically, to those of the arrival, duration and joint occurrence of intermittent load responses arising from various sources such as earthquakes, winds, etc. (See Fig. 1). This problem has attracted considerable attention among engineers and researchers alike, particularly among those working towards developing probabilistically consistent methods of load combinations for nuclear power plant design.

1. Combined Poisson Process Model for Intermittent Load Responses Arising From the Same Source

Consider the following model (henceforth referred to as the combined Poisson process Model) for the arrivals and durations of intermittent load responses. The arrival time is governed by the Poisson arrival law with an arrival rate $\lambda$. The interval $T_{a}$, between two successive arrivals (inter-arrival time), then follows the exponential distribution:

$$
\begin{aligned}
F_{T_{a}}(t) & =1-e^{-\lambda t} & & t>0 \\
& =0 & & \text { otherwise }
\end{aligned}
$$

The duration $T_{d}$ of each intermittent load response is specified by the exponential distribution

$$
\begin{array}{rlrl}
F_{T_{d}}(t) & =1-e^{-(\lambda+\mu) t} & t>0 \\
& =0 & & \text { otherwise }
\end{array}
$$


Equation 2 results from the postulate that the distribution of the duration $T_{d}$ is given by truncating, at $t=T_{a}$, the following exponential distribution of $T_{d}^{\prime}$ with parameter $\mu$.

$$
\begin{aligned}
F_{T_{d}^{\prime}}(t) & =1-e^{-\mu t} & & t>0 \\
& =0 & & \text { otherwise }
\end{aligned}
$$

The distribution of the load duration $T_{d}$ thus defined can be derived in general as

$$
F_{T_{d}}(t)=1-\bar{F}_{T_{d}^{\prime}}(t) \cdot \bar{F}_{T_{a}}(t)
$$

which, under the assumptions of Eqs. 1 and 3, reduces to Eq. 2.

This truncation assures that, as soon as the next intermittent load response arrives, the current load response ceases to exist. However, the underlying process suggested by Eq. 3 is that of a Poisson arrival with arrival rate $\mu$ ( $\mu \gg \lambda$ for practical applications), and hence the name "Combined Poisson Process Model." This model then produces a sequence of intermittent load responses arising from the same source (e.g., an earthquake) without the simultaneous occurrence of two or more responses. This satisfies the general requirement that load responses from the same source not overlap along the time axis.

It follows immediately from Eqs. 1 and 2 that the expected values of $T_{a}$ and $T_{d}$ are

$$
E\left\{T_{a}\right\}=1 / \lambda, \quad E\left[T_{d}\right\}=1 /(\lambda+\mu)
$$

The probabilities $P_{1}$ and $P_{0}$, respectively, of the load response being observed and not being observed at any arbitrary time instant are then given by 


$$
\begin{aligned}
& P_{0}=\left[E\left\{T_{a}\right\}-E\left\{T_{d}\right\}\right] / E\left\{T_{d}\right\}=1 /(1+\rho) \\
& P_{1}=E\left\{T_{d}\right\} / E\left\{T_{a}\right\}=\rho /(1+\rho)
\end{aligned}
$$

where $\rho=\lambda / \mu$ is the parameter known as "traffic intensity" in the queue theory; in the present study, however, this parameter is called the "arrival-duration intensity."

For the further development of the present model, it is instructive to rederive Eqs. 6a and 6b by applying an approach well known in the queue theory: Define $P_{j}(t+\Delta t)$ as the probability of the state of the loading condition being " $i$ " ( $i=0$ implies the response is not being observed and $i=1$, the response is being observed) at the time instant $t+\Delta t$. Then,

or

$$
P_{0}(t+\Delta t)=P_{0}(t)(1-\lambda \Delta t)+P_{1}(t)_{\mu \Delta t}
$$

$$
\begin{gathered}
\dot{P}_{0}=-\lambda P_{0}+\mu P_{1} \\
P_{1}(t+\Delta t)=P_{1}(t)(1-\mu \Delta t)+P_{0}(t) \lambda \Delta t
\end{gathered}
$$

or

$$
\dot{P}_{1}=-\mu P_{1}+\lambda P_{0}
$$

where the dot indicates a time derivative. These equations describe the queue at a single gate processing traffic with an arrival rate of $\lambda$ and service rate $\mu$ (thus, intensity of $\lambda / \mu$ ) when the length $n$ of the queue is limited to $n=1$. Under the steady state condition $\left(\dot{p}_{0}=\dot{p}_{1}=0\right)$, Eqs. $7 \mathrm{~b}$ and $8 \mathrm{~b}$ both result in

$$
\lambda P_{0}-\mu P_{1}=0
$$

which, together with $P_{0}+P_{1}=1$, produces Eqs. 6a and $6 \mathrm{~b}$. 
2. Combined Poisson Process Model for Intermittent Load Responses Arising from Two or More Sources

Those intermittent load responses arising from two sources are considered first. In this case, at any time instant $t$, there exist the following four states in which load response conditions are found.

State I: $\quad$ Both load response 1 and load response 2 are off.

State II: Load response 1 is on and load response 2 is off.

State III: Load response 1 is off and load response 2 is on.

State IV: Both load response 1 and load response 2 are on.

where load response $i$ indicates the intermittent load response arising from source $i(i=1,2)$.

Writing $P_{0}, P_{1}^{(1)}, P_{1}^{(2)}$ and $P_{2}$, respectively, for the probabilities of the load response conditions being in State I, II, III and IV, respectively, we obtain

$$
P_{0}(t+\Delta t)=P_{0}(t)\left[1-\left(\lambda_{1}+\lambda_{2}\right) \Delta t\right]+P_{1}^{(1)}(t)_{\mu_{1} \Delta t}+P_{1}^{(2)}(t)_{\mu_{2} \Delta t}
$$

or

$$
\begin{gathered}
\dot{p}_{0}=-\left(\lambda_{1}+\lambda_{2}\right) p_{0}+\mu_{1} p_{1}^{(1)}+\mu_{2} p_{1}^{(2)} \\
p_{1}^{(1)}(t+\Delta t)=p_{1}^{(1)}(t)\left[1-\left(\mu_{1}+\lambda_{2}\right) \Delta t\right]+p_{0}(t) \lambda_{1} \Delta t+p_{2}(t) \mu_{2} \Delta t
\end{gathered}
$$

or

$$
\dot{P}_{1}^{(1)}=-\left(u_{1}+\lambda_{2}\right) P_{1}^{(1)}+\lambda_{1} p_{0}+u_{2} P_{2}
$$




$$
p_{1}^{(2)}(t+\Delta t)=p_{1}^{(2)}(t)\left[1-\left(\mu_{2}+\lambda_{1}\right) \Delta t\right]+p_{0}(t) \lambda_{2} \Delta t+p_{2}(t) \mu_{1} \Delta t
$$

or

$$
\begin{gathered}
\dot{p}_{1}^{(2)}=-\left(\mu_{2}+\lambda_{1}\right) p_{1}^{(2)}+\lambda_{2} p_{0}+\mu_{1} p_{2} \\
p_{2}(t+\Delta t)=p_{2}(t)\left[1-\left(\mu_{1}+\mu_{2}\right) \Delta t\right]+p_{1}^{(1)}(t) \lambda_{2} \Delta t+p_{1}^{(2)}(t) \lambda_{1} \Delta t
\end{gathered}
$$

or

$$
\dot{p}_{2}=-\left(u_{1}+\mu_{2}\right) p_{2}+\lambda_{2} p_{1}^{(1)}+\lambda_{1} p_{1}^{(2)}
$$

Under the steady state condition, the preceding equations reduce to

$$
\begin{aligned}
& \left(\lambda_{1}+\lambda_{2}\right) P_{0}=\mu_{1} P_{1}^{(1)}+\mu_{2} p_{1}^{(2)} \\
& \left(\mu_{1}+\mu_{2}\right) P_{1}^{(1)}=\lambda_{1} P_{0}+\mu_{2} P_{2} \\
& \left(\mu_{2}+\lambda_{1}\right) P_{1}^{(2)}=\lambda_{2} p_{0}+\mu_{1} P_{2} \\
& \left(\mu_{1}+\mu_{2}\right) P_{2}=\lambda_{2} p_{1}^{(1)}+\lambda_{1} p_{1}^{(2)}
\end{aligned}
$$

Solving these equations for $P_{0}, P_{1}^{(1)}, P_{1}^{(2)}$ and $P_{2}$ under the condition that $P_{0}+P_{1}^{(1)}+P_{1}^{(2)}+P_{2}=1$, we obtain

$$
\begin{aligned}
& P_{0}=1 /\left(1+p_{1}\right)\left(1+p_{2}\right) \\
& p_{1}^{(1)}=p_{1} /\left(1+p_{1}\right)\left(1+p_{2}\right) \\
& P_{1}^{(2)}=p_{2} /\left(1+p_{1}\right)\left(1+p_{2}\right) \\
& P_{2}=p_{1} \rho_{2} /\left(1+p_{1}\right)\left(1+p_{2}\right)
\end{aligned}
$$


where the $\rho$ 's are the arrival-duration intensities such that $\rho_{1}=\lambda_{1} / \mu_{1}$ and $p_{2}=\lambda_{2} / \mu_{2}$.

When the intermittent load responses arising from $N$ sources are considered, the results shown in Eqs. 15a - 15d can be generalized so that

$$
\begin{aligned}
& P_{0}=1 / \prod_{m=1}^{N}\left(1+\rho_{m}\right) \\
& p_{1}^{(i)}=\rho_{i} / \prod_{m=1}^{N}\left(1+\rho_{m}\right) \\
& p_{2}^{(i, j)}=\rho_{i} \rho_{j} / \prod_{m=1}^{N}\left(1+\rho_{m}\right) \\
& p_{3}^{(i, j, k)}=\rho_{i} \rho_{j} \rho_{k} / \underset{m=1}{N}\left(1+\rho_{m}\right) \quad i \neq j \quad k \neq i \quad j \neq k \\
& P_{N}=\underset{n=1}{N} \rho_{n} / \underset{m=1}{N}\left(1+\rho_{m}\right)
\end{aligned}
$$

where $\rho_{i}=\lambda_{i} / \mu_{i}$ with $\mu_{i}=$ the arrival rate of load response $i$ and $\lambda_{i}=$ the duration rate of load response $i$ and

$P_{0}=$ the probability that none of the load responses are on.

$$
\begin{aligned}
& p_{1}^{(i)}=\text { the probability that load response } i \text { and only load response } \\
& i \text { is on. }
\end{aligned}
$$
$p_{2}^{(i, j)}=$ the probability that load response $i$ and load response $j$ are simultaneously on.

$p_{3}^{(i, j, k)}=$ the probability that load responses $i, j$ and $k$ are 


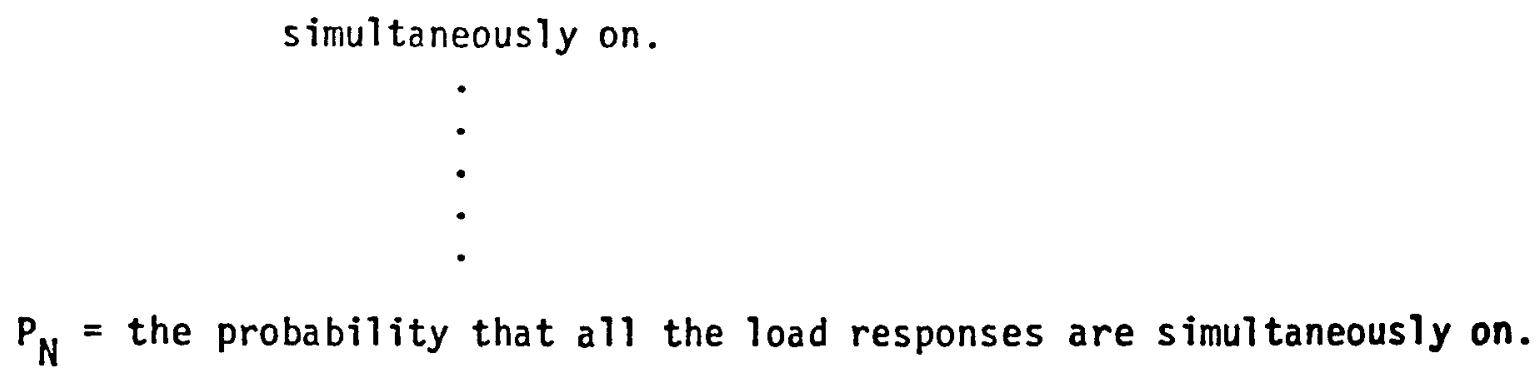

3. Probability of Load Responses Exceeding Specified Levels

Many researchers have endeavored to solve the problem of evaluating the probability of a random process exceeding specified levels in a given time interval. M. Shinozuka was one of the first to study this problem in the context of the analysis of structural safety and reliability. The methods described here are based on the work by Shinozuka ${ }^{1}$ and Shinozuka and Yao ${ }^{2}$.

Let $x(t)$ be a random load response process which is not necessarily stationary. Also, let $\left\{T ; x \leqq-\alpha_{1}\right\}$ denote the event that the process $x(t)$ will take values less than or equal to $-\alpha_{1}(t)$ at least once in the time interval $[0, T]$ and similarly, $\left\{T ; \quad X \geqq \dot{\alpha}_{2}\right\}$ denote the event that $X(t)$ will take values larger than or equal to $\alpha_{2}(t)$ at least once in the same time interval, where $\alpha_{1}(t)$ and $\alpha_{2}(t)$ are both positive and deterministic functions of the time $t$. Then, the union of these two events, $\left\{T ; x \leq-\dot{\alpha}_{1}\right\} \quad\left[T ; \quad X \geq \dot{\alpha}_{2}\right\}$ represents the event that $x(t)$ will not be confined within the two barriers $-\dot{\alpha}_{1}(t)$ and $\alpha_{2}(t)$ in the time interval $[0, T]$. Therefore, if the barriers $-\alpha_{1}(t)$ and $\alpha_{2}(t)$ indicate the structural response capacities in the negative and positive ranges, respectively, the probability of the structural failure $P_{X}\left(T ;-d_{1}, d_{2}\right)$ during the time interval $[0, T]$ is given by

$$
P_{X}\left(T ;-\alpha_{1}, \alpha_{2}\right)=P\left[\left\{T ; \quad X \leq-\alpha_{1}\right\} \cup\left\{T ; X \geq \alpha_{2}\right\}\right]
$$


If both barriers $-\alpha_{1}(t)$ and $\alpha_{2}(t)$ are finite, the probability $P_{X}\left(T ;-\alpha_{1}, \alpha_{2}\right)$ is referred to as the first excursion failure probability with a two-sided barrier, while it is a first excursion failure probability with a one-sided barrier if one of the barriers is infinite and the other is finite.

It can be shown that, for any time instant $t_{1}\left(0 \leq t_{1} \leq T\right)$, the event $A_{1}\left(t_{1}\right)$ that $x\left(t_{1}\right)$ is less than or equal to $-\alpha_{1}\left(t_{1}\right)$ is a subset of the event $\left\{T ; \quad X \leq-\alpha_{1}\right\}$ defined above:

$$
A_{1}\left(t_{1}\right) \equiv\left\{X\left(t_{1}\right) \leq-a_{1}\left(t_{1}\right)\right\} \subset\left\{T ; \quad X \leq-\alpha_{1}\right\}
$$

Similarly, for $t_{2}$ in the interval $[0, T]$,

$$
A_{2}\left(t_{2}\right) \equiv\left\{X\left(t_{2}\right) \geqq \alpha_{2}\left(t_{2}\right)\right\} \subset\left\{T ; \quad X \geqq \alpha_{2}\right\}
$$

It then follows from Eq. 17 that

$$
\begin{aligned}
P_{X}\left(T ;-\dot{\alpha}_{1}, \dot{\alpha}_{2}\right)>P\left\{A_{1}\left(t_{1}\right) \cup A_{2}\left(t_{2}\right)\right\} & =P\left[A_{1}\left(t_{1}\right)\right]+P\left[A_{2}\left(t_{2}\right)\right] \\
& -P\left[A_{1}\left(t_{1}\right) \cap A_{2}\left(t_{2}\right)\right]
\end{aligned}
$$

The last member of Eq. 20 is a lower bound of $P_{X}\left(T ;-a_{1}, \alpha_{2}\right)$. The time instants $t_{1}$ and $t_{2}$ should be chosen so that the right-hand side of Eq. 20 achieves its largest possible value, if possible.

With the aid of the addition rule of the proability theory, Eq. 17 can be expanded into the following form:

$$
\begin{aligned}
P_{X}\left(T ;-\alpha_{1}, \alpha_{2}\right) & =P\left\{\left\{T ; \quad X \leq-\alpha_{1}\right\} \cup\left\{T ; \quad X \geq \alpha_{2}\right\}\right] \\
& =P\left\{T ; \quad X \leqq-\alpha_{1}\right\}+P\left\{T ; \quad X \geqq \alpha_{2}\right\} \\
& -P\left\{\left\{T ; \quad X \leq-\alpha_{1}\right\} \cap\left\{T ; \quad X \geqq \alpha_{2}\right\}\right]
\end{aligned}
$$


which, with the further aid of Eqs. 18 and 19, becomes

$$
P_{X}\left(T ;-\alpha_{1}, \alpha_{2}\right)<P\left\{T ; \quad X \leq-\alpha_{1}\right\}+P\left\{T ; X \geq \alpha_{2}\right\}-P\left\{A_{1}\left(t_{1}\right) \cap A_{2}\left(t_{2}\right)\right\}
$$

where $n$ indicates the intersection. The right-hand side of Eq. 22 is an upper bound of $P_{X}\left(T ;-\alpha_{1}, \alpha_{2}\right)$. Since it is an upper bound, the last member may be disregarded to establish another upper bound of the simpler form

$$
P_{X}\left(T ;-\alpha_{1}, \alpha_{2}\right)<P\left\{T ; \quad X \leq-\alpha_{1}\right\}+P\left\{T ; \quad X \geq \alpha_{2}\right\}
$$

It can be shown ${ }^{2}$ that

$$
\begin{aligned}
& \frac{d P\left\{T ; \quad X \geqq \alpha_{2}\right\}}{d T}<\int_{\dot{\alpha}_{2}}^{\infty}\left(\dot{X}-\dot{\alpha}_{2}\right) \phi\left(\alpha_{2}, \dot{X} ; T\right) d \dot{X} \equiv h_{2}(T) \\
& \frac{d P\left\{T ; \quad X \leqq-\alpha_{1}\right\}}{d T}<\int_{\infty}^{-\dot{\alpha}_{1}}\left(-\dot{\alpha}_{1}-\dot{X}\right)_{\phi}\left(\alpha_{1}, X ; T\right) d \dot{X} \equiv h_{1}(T)
\end{aligned}
$$

where $\phi(X, \dot{X} ; T)$ is the joint density function of $X(T)$ and $\dot{X}(T)$, and $h_{2}(T)$ and $h_{1}(T)$ are the rates (at time $T$ ) of the upcrossing of the level $\alpha_{2}(t)$ and of the downcrossing of the level $-\alpha_{1}(t)$ by the process $x(t)$, respectively. Integrating Eqs. 24a and 24b and using Eq. 22, we obtain

$$
\begin{aligned}
P_{X}\left(T ;-\alpha_{1}, \alpha_{2}\right) & <\int_{0}^{T}\left[h_{1}(t)+h_{2}(t)\right] d t+P\left\{X(0) \leq-\alpha_{1}(0)\right\} \\
& +P\left\{X(0) \geqq \dot{\alpha}_{2}(0)\right\}-P\left\{A_{1}\left(t_{1}\right) \cap A_{2}\left(t_{2}\right)\right\}
\end{aligned}
$$

where the initial failure probabilities $P\left\{X(0) \leq-\alpha_{1}(0)\right\}$ and $P\left\{X(0) \geq \alpha_{2}(0)\right\}$ 
enter into the expression through the initial condition. As before, even with the last member of the right-hand side of Eq. 25a disregarded, we still have an upper bound:

$$
\begin{aligned}
P_{X}\left(T ;-\alpha_{1}, \alpha_{2}\right) & <\int_{0}^{T}\left[h_{1}(t)+h_{2}(t) d t+P\left\{X(0) \leq-\alpha_{1}(0)\right\}\right. \\
& +P\left\{X(0) \geqq \alpha_{2}(0)\right\}
\end{aligned}
$$

Furthermore, if the zero initial condition $(x(0) \equiv 0)$ is assumed, then the initial failure probabilities are zero and therefore

$$
P_{X}\left(T ;-\alpha_{1}, \alpha_{2}\right)<\int_{0}^{T}\left[h_{1}(t)+h_{2}(t)\right] d t
$$

It is noted that the initial failure probabilities are often negligibly small when compared with the intergration of the crossing rate over the time interval and therefore Eq. $25 \mathrm{c}$ can be used even under non-zero initial conditions.

If $X(t)$ is stationary and both barriers $-\alpha_{1}$ and $\alpha_{2}$ are constant, then $h(t)=h_{1}, h_{2}(t)=h_{2}, P\left\{X(0) \leq-\alpha_{1}\right\}=P\left\{A_{1}\left(t_{1}\right)\right\}=A_{01}$ and $P\left\{X(0) \geq \alpha_{2}\right\}=$ $P\left\{A_{2}\left(t_{2}\right)\right\}=A_{02}$ are all time invariant. Furthermore, by choosing $t_{1}=t_{2}$, we obtain $P\left\{A_{1}\left(t_{1}\right) \cap A_{2}\left(t_{2}\right)\right\}=0$. Therefore,

$$
A_{01}+A_{02}<P_{X}\left(T ;-\alpha_{1}, \dot{\alpha}_{2}\right)<\left(h_{1}+h_{2}\right) T+A_{01}+A_{02}
$$

The lower and upper bounds indicated in Eqs. 20 and 25a represent the most significant result of the work by Shinozuka and Yao ${ }^{2}$, while the upper and lower bounds associated with the case where $\alpha_{1}$ and $\alpha_{2}$ are both positive constants (for which the first and last members of Eq. 26 are special cases) were initially derived by Shinozuka ${ }^{1}$. As emphasized by Shinozuka himself and later recognized by $Y . K$. Lin $^{3}$, these bounding techniques are especially. suited for evaluating the first excursion probability under the condition that 
the response is relatively intense only within a short duration of time. Indeed, the numerical examples presented by Shinozuka ${ }^{1,4}$ and Shinozuka et al 5 dealing with single- and multiple-degree-of-freedom systems, respectively, subjected to nonstationary Gaussian earthquake-like random processes, verify that the bounds evaluated on the basis of Eqs. 20 and 25a are close enough to be of practical use under the conditions just mentioned. In the context of the present application, however, this implies that during each occurrence of the intermittent load, the response must be relatively intense within a short period of time for the bounding techniques to produce practical results.

\section{Probability of Structural Failure under Intermittent Load Responses Arising}

As a first approximation, consider that the structural response capacities are $-\alpha_{1}$ (constant) and $\alpha_{2}$ (constant), respectively, in the negative and posttive ranges. Furthermore, assume that the upcrossing rate $h_{1}(t)$ and the downcrossing rate $h_{2}(t)$ are both time invariant and hence the rate of crossing

$$
v(t)=h_{1}(t)+h_{2}(t)
$$

is also time invariant. This assumption further implies that the intermittent load response process $x(t)$ is stationary while it is on, or, to be more precise, one occurrence of $x(t)$, initiated at time $t$ and terminated at time $t+T$, can be modeled in terms of a stationary random process which is set equal to zero except for that segment in the time interval $[t, t+T]$ where it is on.

We shall consider a structure subjected to (statistically independent) intermittent load responses arising from $N$ different sources, to each of which the same assumptions introduced above apply. Let 


$$
\begin{aligned}
v_{i}(t)= & v_{i} \text { (constant)= rate of crossing by the load response } \\
& i, x_{i}(t) \text {, when } x_{i}(t) \text { is the only load response that is } \\
& \text { on. } \\
v_{i+j}(t)= & v_{i+j} \text { (constant) }=\text { rate of crossing by the sum of the } \\
& \text { load responses } x_{j}(t) \text { and } x_{j}(t)(i \neq j) \text { when } x_{i}(t) \text { and } \\
& x_{j}(t) \text { are the only load responses that are on simul- } \\
& \text { taneously. }
\end{aligned}
$$

Similar definitions apply to $v_{i+j+k}(t)=v_{i+j+k}=$ constant $(i \neq j, j \neq k, k \neq i)$, etc. Then, an upper bound for the probability of structural failure due to the first excursion into the time interval $[0, T]$ is given by

$$
\begin{gathered}
T\left\{\sum_{i=1}^{N} p_{1}^{(i)} v_{i}+\sum_{\substack{i \neq j \\
i \neq j}}^{N} p_{2}^{(i, j)} v_{i+j}+\sum_{\substack{i \neq j \\
i \neq j}}^{N} \sum_{\substack{j \neq j \\
k \neq i}}^{N} \sum_{3}^{N} p_{3}^{(i, j, k)} v_{i+j+k}\right. \\
\left.+\ldots+P_{N} v_{1+2+\cdots+N}\right\}
\end{gathered}
$$

where $p_{1}^{(i)}, p_{2}^{(i, j)}, p_{3}^{(i, j, k)}, \ldots, p_{N}$ are defined in Eqs. 16a - 16e.

The crossing rates $v_{i+j}, v_{i+j+k}, \ldots, v_{1+2+\cdots+N}$ may be established with the aid of the technique developed by Winterstein ${ }^{6}$.

\section{ACKNOWLEDGEMENT}

This work was supported by Brookhaven National Laboratory under Grant No. BNL-517722-S. 


\section{REFERENCES}

1. Shinozuka, M., "Probability of Structural Failure under Random Loading," Journal of the Engineering Mechanics Division, ASCE, Vor. 90, No. EN5, October 1964, pp. 147-170.

2. Shinozuka, M. and Yao, J.T.P., "On the Two-Sided Time-Dependent Barrier Problem," Journal of Sound and Vibration, Vol. 6, No. 1, 1967, pp. 98-104.

3. Lin, Y.K., Probabilistic Theory of Structural Dynamics, McGraw-Hill, New York, 1967, pp. 322-332.

4. Shinozuka, M., "Probability of Structural Failure under Random Loading: Closure to Discussion," Journal of the Engineering Mechanics Division, ASCE, Vol. 92, No. EMI, February 1966, pp. 190-196.

5. Shinozuka, M., Itagaki, H. and Hakuno, M., "Dynamic Safety Analysis of Multistory Buildings," Journal of the Structural Division. ASCE, Vol. 94, No. ST1, January 1968, pp. 309-330.

6. Winterstein, S.R., "Stochastic Dynamic Response Combinations," M.S. dissertation, Massachusetts Institute of Technology, May 1980. 


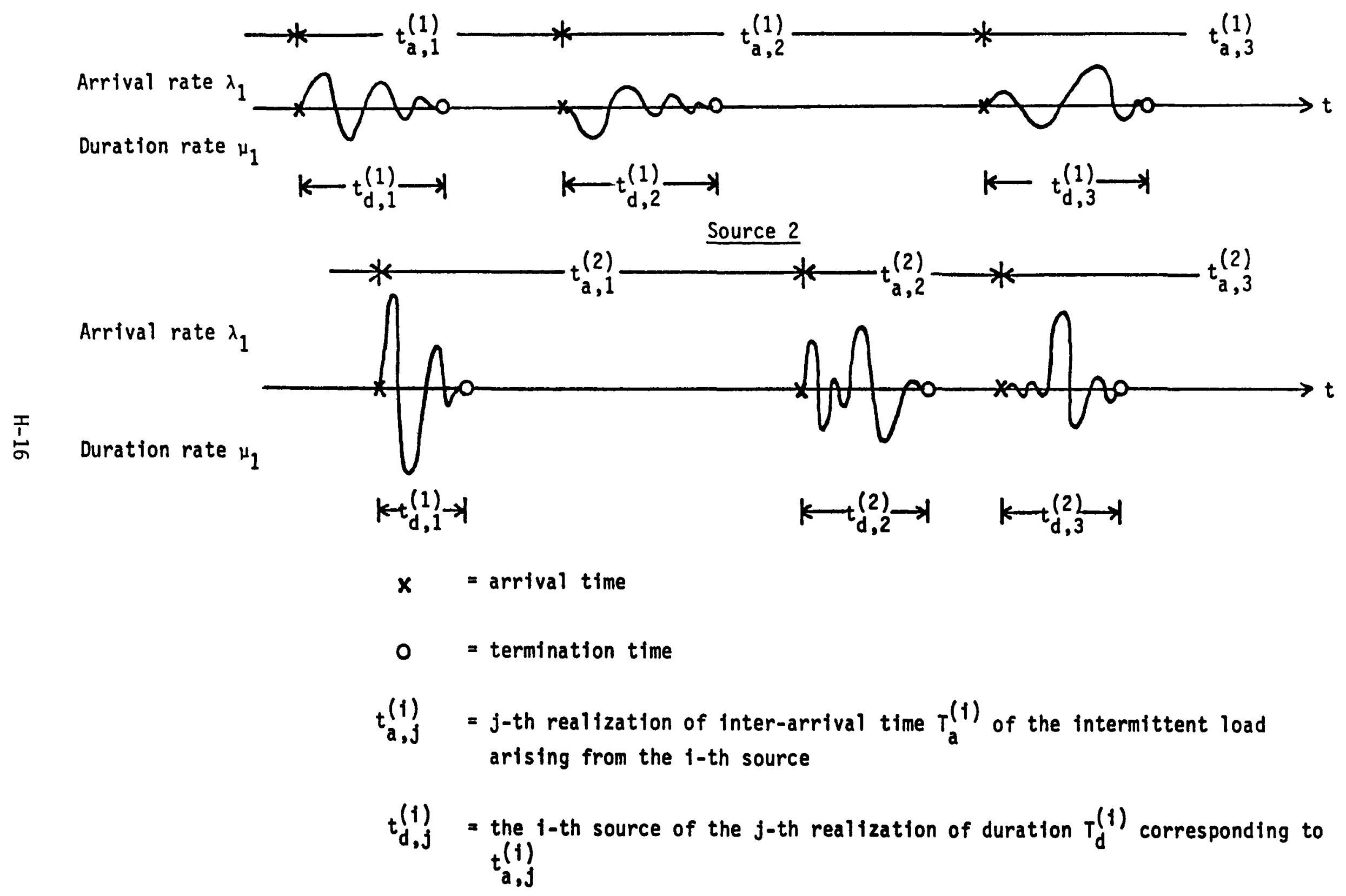


Appendix I

Notes on the Combinations of Random Loads II 
NOTES ON THE COMBINATIONS OF RANDOM LOADS (II)

by

M. Shinozuka

Renwick Professor of Civil Engineering

R. Tan

Graduate Research Assistant

Department of Civil Engineering and Engineering Mechanics Columbia University

New York, N.Y.

August 1980 
TABLE OF CONTENTS

page

1. Combined Poisson and Erlang Model for Intermittent Load Responses Arising from the Same Source 1

2. Combined Poisson and Erlang Model for Intermittent Load Responses Arising from Two or More Sources

4

3.

Probability of Structural Failure under Intermittent

Load Responses Arising from Various Sources

7

References 
1. Combined Poisson and Erlang Model for Intermittent Load Responses Arising from the Same Source

Consider the following model for the arrivals and durations of intermittent load responses. The arrival time is governed by the Poisson arrival law with an arrival rate $\lambda$. The interval $T_{a}$ between two successive arrivals (inter-arrival time) then follows the following exponential distribution:

$$
\begin{aligned}
F_{T_{a}}(t) & =1-e^{-\lambda t} & & t>0 \\
& =0 & & \text { otherwise }
\end{aligned}
$$

The duration $T_{d}$ of each intermittent load is specified by

$$
\begin{array}{rlrl}
F_{T_{d}}(t) & =1-e^{-\lambda t}\left[1-\int_{0}^{t} g(t) d t\right] t>0 \\
& =0 & \text { otherwise }
\end{array}
$$

where $g(t)$ is an Erlang probability density function:

$$
g(t)=(k \mu)^{k} t^{k-1} e^{-k \mu t} /(k-1) !
$$

with parameters $\mu$ and $k$. The parameter $k$ represents the total number of component exponential durations (each with parameter $\mu$ ) involved in the Erlang duration.

Equation 2 follows from the postulate that the distribution of the duration is given by truncating an Erlang distribution with parameters $\mu$ and $k$ at $t=T_{a}$. This truncation assures that, as soon as the next intermittent load arrives, the current load ceases to exist. This model then produces a sequence of intermittent loads arising from the same source (e.g., an earthquake) without the simultaneous occurrence of two or more loads as is usually the case in reality. It follows immediately from Eq. I that the expected values of $T_{a}$ is 


$$
E\left[T_{a}\right]=\frac{1}{\lambda}
$$

while the expected value of $T_{d}$ can be obtained from Eq. 2 as

$$
E\left[T_{d}\right]=\int_{0}^{t} t f_{T_{d}}(t) d t=\frac{1}{\lambda}\left[1-\frac{1}{(1+\rho)^{k}}\right]
$$

where $f_{T_{d}}(t)=d F_{T_{d}}(t) / d t$ is the probability density function of $T_{d}$ and $\rho=\lambda / k \mu$ is referred to as the arrival-duration intensity similarly to the traffic intensity in the queue theory.

Let $P_{0}(t)$ (or $P_{0}$ for simplicity) represent the probability of load response "not being observed" at any arbitrary time instant $t$ and let $P_{i}(t)$ (or $\left.P_{i}\right)(i=1,2, \ldots, k)$ denote the probabilities of load response "being observed (while it is) in the phase $i$ " at time $t$. The phase $i$ indicates the period of the $i$-th component exponential duration counting from the last. Then, it immediately follows that

$$
\begin{array}{r}
P_{0}=\left\{E\left[T_{a}\right]-E\left[T_{d}\right]\right\} / E\left[T_{a}\right]=\frac{1}{(1+p)^{k}} \\
P_{1}+P_{2}+\ldots+P_{k}=E\left[T_{d}\right] / E\left[T_{a}\right]=1-\frac{1}{(1+p)^{k}}
\end{array}
$$

These equations can alternatively be derived from the queue theory. Assume, for example, that $k=3$. Then

$$
P_{0}(t+\Delta t)=P_{0}(t)(1-\lambda \Delta t)+P_{1}(t)(1-\lambda \Delta t) k \mu \Delta t
$$

or

$$
\begin{gathered}
\dot{p}_{0}=-\dot{\lambda}_{0}+k \mu P_{1} \\
P_{1}(t+\Delta t)=P_{1}(t)(1-\lambda \Delta t)(1-k \mu \Delta t)+P_{2}(1-\lambda \Delta t) k \mu \Delta t
\end{gathered}
$$

or

$$
\dot{p}_{1}=-(\lambda+k \mu) P_{1}+k \mu p_{2}
$$




$$
P_{2}(t+\Delta t)=P_{2}(t)(1-\lambda \Delta t)(1-k \mu \Delta t)+P_{3}(t)(1-\lambda \Delta t) k \mu \Delta t
$$

or

$$
\begin{aligned}
\dot{P}_{2} & =-(\lambda+k u) P_{2}+k \mu P_{3} \\
P_{3}(t+\Delta t)= & P_{3}(t)(1-\lambda \Delta t)(1-k \mu \Delta t) \\
& +\left(P_{0}+P_{1}+P_{2}+P_{3}\right) \lambda \Delta t(1-k \mu \Delta t)
\end{aligned}
$$

or

$$
\dot{P}_{3}=\lambda\left(P_{0}+P_{1}+P_{2}\right)-\mu k P_{3}
$$

Under the steady state condition, Eqs. 7a - 7d, together with $P_{0}+P_{1}+P_{2}+P_{3}=1$, produce

$$
\begin{aligned}
& P_{0}=\frac{1}{(1+\rho)^{3}} \\
& P_{1}=\frac{\rho}{(1+\rho)^{3}} \\
& P_{2}=\frac{\rho(1+\rho)}{(1+\rho)^{3}} \\
& P_{3}=\frac{\rho(1+\rho)^{2}}{(1+\rho)^{3}}
\end{aligned}
$$

Equations $6 a$ and $6 \mathrm{~b}$ follow immediately from Eq. 8. Equation 8 can easily be generalized so that

$$
\begin{aligned}
& P_{0}=\frac{1}{(1+\rho)^{k}} \\
& P_{i}=\frac{\rho(1+\rho)^{i-1}}{(1+\rho)^{k}} \quad 0<i \leq k
\end{aligned}
$$




\section{Combined Poisson and Erlang Model for Intermittent Load Responses Arising from Two or More Sources}

These intermittent load responses arising from two sources are considered first. The load response (from source) $i$ arrives with arrival rate $\lambda_{i}$ with duration distributed in accordance with an Erlang distribution with parameters $\mu_{i}$ and $k_{i}(i=1,2)$. Let $P_{i j}(t)$ or $P_{i, j}(t)$ represent the probability of load response 1 being observed "in phase $i$ " and load response 2 "in phase $j "$ at any arbitrary time instant $t$. By definition, phase zero indicates the event that the corresponding load response is not being observed. Then, the following relationships can be derived

$$
\begin{array}{rlrl}
P_{1 j}(t+\Delta t) & =P 1+P 2+P 3 & 0 \leq i<k_{1}, 0 \leq j<k_{2} \\
& =P 1+P 4+P 3 & i=k_{1}, 0 \leq j<k_{2} \\
& =P 1+P 2+P 5 & 0 \leq i<k_{1}, \quad j=k_{2} \\
& =P 1+P 4+P 5 & i=k_{1}, \quad j=k_{2}
\end{array}
$$

where

$$
\begin{aligned}
& P 1=P_{i j}(t) \cdot\left(1-\lambda_{1} \Delta t\right)\left(1-c_{i} k_{1} \mu_{1} \Delta t\right)\left(1-\lambda_{2} \Delta t\right)\left(1-c_{j} k_{2} \mu_{2} \Delta t\right) \\
& P 2=P_{i+1, j}(t) \cdot\left(1-\lambda_{1} \Delta t\right) k_{1} \mu_{1} \Delta t\left(1-\lambda_{2} \Delta t\right)\left(1-c_{j} k_{2} \mu_{2} \Delta t\right) \\
& P 3=P_{i, j+1}(t) \cdot\left(1-\lambda_{1} \Delta t\right)\left(1-c_{i} k_{1} \mu_{1} \Delta t\right)\left(1-\lambda_{2} \Delta t\right) k_{2} \mu_{2} \Delta t \\
& P 4=P_{k_{1}+1, j}(t) \cdot \lambda_{1} \Delta t\left(1-k_{1} \mu_{1} \Delta t\right)\left(1-\lambda_{2} \Delta t\right)\left(1-c_{j} k_{2} \mu_{2} \Delta t\right) \\
& P 5=P_{i, k_{2}+1}(t) \cdot\left(1-\lambda_{1} \Delta t\right)\left(1-c_{i} k_{1} \mu_{1} \Delta t\right) \lambda_{2} \Delta t\left(1-k_{2} \mu_{2} \Delta t\right)
\end{aligned}
$$

with

$$
c_{i}=0 \text { if } i=0, c_{i}=1 \text { if } i \neq 0
$$

and

$$
\begin{aligned}
& P_{k_{1}+1, j}=P_{0 j}+P_{1 j}+P_{2 j}+\cdots+P_{k_{1} j} \\
& P_{i, k_{2}+1}=P_{i 0}+P_{i 1}+P_{i 2}+\cdots+P_{i k_{2}}
\end{aligned}
$$


Before we find the general expression of $P_{i j}$, we first assume $k_{1}=3$ and $k_{2}=2$. Using Eq. 10 under the steady state conditions, we obtain a set of $\left(k_{1}+1\right)\left(k_{2}+2\right)$ simultaneous equations:

$$
\begin{aligned}
& -\left(\lambda_{1}+\lambda_{2}\right) P_{00}+k_{1} \mu_{1} P_{10}+k_{2} \mu_{2} P_{01}=0 \\
& -\left(\lambda_{1}+k_{1} \mu_{1}+\lambda_{2}\right) P_{10}+k_{1} \mu_{1} P_{20}+k_{2} \mu_{2} P_{11}=0 \\
& -\left(\lambda_{1}+k_{1} \mu_{1}+\lambda_{2}\right) P_{20}+k_{1} \mu_{1} P_{30}+k_{2} \mu_{2} p_{21}=0 \\
& -\left(k_{1} \mu_{1}+\lambda_{2}\right) P_{30}+\lambda_{1}\left(P_{00}+P_{10}+P_{20}\right)+k_{2} \mu_{2} P_{31}=0 \\
& -\left(\lambda_{1}+\lambda_{2}+k_{2} \mu_{2}\right) P_{01}+k_{1} \mu_{1} P_{11}+k_{2} \mu_{2} P_{02}=0 \\
& -\left(\lambda_{1}+k_{2} \mu_{2}\right) P_{02}+k_{1} \mu_{1} p_{12}+\lambda_{2}\left(P_{00}+P_{01}\right)=0 \\
& -\left(\lambda_{1}+k_{1} \mu_{1}+\lambda_{2}+k_{2} \mu_{2}\right) p_{11}+k_{1} \mu_{1} p_{21}+k_{2} \mu_{2} p_{12}=0 \\
& -\left(\lambda_{1}+k_{1} \mu_{1}+k_{2} \mu_{2}\right) P_{12}+k_{1} \mu_{1} P_{22}+\lambda_{2}\left(P_{10}+P_{11}\right)=0 \\
& -\left(\lambda_{1}+k_{1} \mu_{1}+\lambda_{2}+k_{2} \mu_{2}\right) P_{21}+k_{1} \mu_{1} P_{31}+k_{2} \mu_{2} P_{22}=0 \\
& -\left(\lambda_{1}+k_{1} \mu_{1}+k_{2} \mu_{2}\right) P_{22}+k_{1} \mu_{1} P_{32}+\lambda_{2}\left(P_{20}+P_{21}\right)=0 \\
& -\left(k_{1} \mu_{1}+\lambda_{2}+k_{2} \mu_{2}\right) P_{31}+\lambda_{1}\left(P_{01}+P_{11}+P_{21}\right)+k_{2} \mu_{2} P_{32}=0 \\
& -\left(\lambda_{1}+k_{1} \mu_{1}+k_{2} \mu_{2}\right) P_{32}+\lambda_{1}\left(P_{01}+P_{11}+P_{21}+P_{31}\right) \\
& +\lambda_{2}\left(P_{30}+P_{31}\right)=0
\end{aligned}
$$

Note that Eqs. (14a) to (14l) are not linearly independent and the rank of the corresponding coefficient matrix is 11 . However, one more equation to satisfy:

$$
\sum_{i=0}^{k_{1}} \sum_{j=0}^{k_{2}} P_{i j}=1
$$

Thus, we can solve Eqs. $14 a-14 k$ and Eq. 15 for $P_{i j}$. The results are 


$$
\begin{aligned}
& P_{00}=1 / \Delta \\
& P_{10}=\rho_{1} / \Delta \\
& P_{20}=\rho_{1}\left(1+\rho_{1}\right) / \Delta \\
& P_{30}=\rho_{1}\left(1+\rho_{1}\right)^{2} / \Delta \\
& P_{01}=\rho_{2} / \Delta \\
& P_{02}=\rho_{2}\left(1+\rho_{2}\right) / \Delta \\
& P_{11}=\rho_{1} \rho_{2} / \Delta \\
& P_{12}=\rho_{1} \rho_{2}\left(1+\rho_{2}\right) / \Delta \\
& P_{21}=\rho_{1}\left(1+\rho_{1}\right) \rho_{2} / \Delta \\
& P_{22}=\rho_{1}\left(1+\rho_{1}\right) \rho_{2}\left(1+\rho_{2}\right) / \Delta \\
& P_{31}=\rho_{1}\left(1+\rho_{1}\right)^{2} \rho_{2} / \Delta \\
& P_{32}=\rho_{1}\left(1+\rho_{1}\right)^{2} \rho_{2}\left(1+\rho_{2}\right) / \Delta
\end{aligned}
$$

where

$$
p_{1}=\lambda_{1} / k_{1} \mu_{1}, \quad p_{2}=\lambda_{2} / k_{2} \mu_{2}, \Delta=\left(1+p_{1}\right)^{3}\left(1+p_{2}\right)^{2}
$$

For arbitrary $k_{1}, k_{2}$, Eq. 16 can be generalized as

$$
\begin{array}{rlrl}
P_{i j} & =1 /\left(1+\rho_{1}\right)^{k}\left(1+p_{2}\right)^{k_{2}} & i=0, j=0 \\
& =p_{1}\left(1+\rho_{1}\right)^{i-1 / \Delta} & k_{1} \geq 1 \neq 0, j=0 \\
& =p_{2}\left(1+\rho_{2}\right)^{j-1} / \Delta & i=0, k_{2} \geq j \neq 0 \\
& =p_{1}\left(1+\rho_{1}\right)^{i-1} \rho_{2}\left(1+p_{2}\right)^{j-1} / \Delta & k_{1} \geq 1 \neq 0, k_{2} \geq j \neq 0
\end{array}
$$

(18)

with

$$
\Delta=\left(1+\rho_{1}\right)^{k} 1\left(1+\rho_{2}\right)^{k_{2}}
$$

I-9 
When the intermittent load responses arising from $N$ sources are considered, the results shown in Eq. 18 can further be generalized as

$$
\begin{aligned}
& P_{i_{1} i_{2} \ldots \ldots i_{N}}=1 / \Delta \\
& =\rho_{1}\left(1+\rho_{1}\right)^{i_{1}-1} / \Delta \\
& i_{1}=t_{2}=\cdots=t_{N}=0 \\
& =\rho_{1}\left(1+\rho_{1}\right)^{i} 1-1 \rho_{2}\left(1+\rho_{2}\right)^{i_{2}-1} / \Delta \\
& k_{1} \geq i_{1} \neq 0, k_{2} \geq i_{2} \neq 0, i_{3}=i_{4}=\ldots=i_{N}=0 \\
& =\rho_{1}\left(1+\rho_{1}\right)^{i_{1}-1} \rho_{3}\left(1+\rho_{3}\right)^{i_{3}-1} / \Delta \\
& k_{1} \geq i_{1} \neq 0, \quad k_{3} \geq i_{3} \neq 0, \quad i_{2}=i_{4}=\ldots .=i_{N}=0 \\
& \vdots \\
& =\rho_{1}\left(1+\rho_{1}\right)^{i} \rho^{-1} \rho_{2}\left(1+\rho_{2}\right)^{i} 2^{-1} \ldots \ldots \rho_{N}\left(1+\rho_{N}\right)^{i_{N}-1} / \Delta \\
& k_{1} \geq i_{1} \neq 0, \quad k_{2} \geq i_{2} \neq 0, \cdots, k_{N} \geq i_{N} \neq 0
\end{aligned}
$$

with

$$
\Delta=\prod_{j=1}^{N}\left(1+\rho_{j}\right)^{k_{j}}
$$

3. Probability of Structural Failure under Intermittent Load Responses Arising From Various Sources

We now consider a structure subjected to intermittent load responses arising from $N$ different sources which are statistically independent. Let ${ }^{1}$

$$
\begin{aligned}
v_{i}(t)= & v_{i} \text { (constant) }=\text { rate of crossing by the load response } \\
& i, x_{i}(t) \text {, when } x_{i}(t) \text { is the only load response that is } \\
& \text { on. }
\end{aligned}
$$




$$
\begin{aligned}
v_{i+j}(t)= & v_{i+j} \text { (constant) }=\text { rate of crossing by the sum of the } \\
& \text { load responses } x_{j}(t) \text { and } x_{j}(t)(i \neq j) \text { when } x_{i}(t) \text { and } \\
& x_{j}(t) \text { are the only load responses that are on simul- } \\
& \text { taneousiy. }
\end{aligned}
$$

Similar definitions apply to $v_{i+j+k}(t)=v_{j+j+k}=$ constant $(i \neq j, j \neq k, k \neq i)$, d. Then, an upper bound for the probability of structural failure due to the finst excursion in the time interval $[0, T]$ is given by

$$
\begin{aligned}
& T\left\{\left[v_{1} \sum_{i_{1}}^{k_{1}} P_{i_{1}, 0, \cdots, 0}+v_{2} \sum_{i_{2}}^{k_{2}} P_{0, i_{2}, 0, \cdots, 0}+v_{N} \sum_{i_{N}}^{k_{N}} P_{0, \cdots, 0, i_{N}}\right]\right. \\
& +\left[v_{1+2} \sum_{i_{1}}^{k_{1}} \sum_{i_{2}}^{k_{2}} P_{i_{1}, i_{2}, 0, \cdots, 0}+\cdots \cdots+v_{(N-1)+N} \sum_{i_{N-1}}^{k_{N-1}} \sum_{i_{N}}^{k_{N}} P_{0, \cdots, 0, i_{N-1}, i_{1}}\right. \\
& +\left[v_{1+2+3} \sum_{i_{1}}^{k_{1}} \sum_{i_{2}}^{k_{2}} \sum_{i_{3}}^{k_{3}} P_{i_{1}, i_{2}, i_{3}, 0, \cdots, 0}+\cdots \cdots+\right. \\
& \left.{ }^{v}(N-2)+(N-1)+N i_{N-2}^{k_{N-2}} \sum_{i_{N-1}}^{k_{N-1}} \sum_{i_{N}}^{k_{N}} P_{0}, \cdots, 0, i_{N-2}, i_{N-1}, i_{N}\right] \\
& \left.+\left[v_{1+2+\cdots \cdots+N} \sum_{i_{1}}^{k_{1}} \sum_{i_{2}}^{k_{2}} \cdots \sum_{i_{N}}^{k_{N}} P_{i_{1}, i_{2}}, \cdots i_{N}\right]\right\}
\end{aligned}
$$

Although Eq. 22 is lengthy, it is a straightforward extension of Eq. 28 of Ref1 which deals with the Combined Poisson Process Model. The sum of the terms 
the first pair of square brackets indicates the failure probability attributable to the case where only one load response is acting. The sum of the terms in the second pair is equal to the failure probability resulting from the combination of any two loads. Obviously, there are ${ }_{N} C_{2}$ load combinations and therefore there are that many terms in the second pair of brackets. Similar interpretations can be applied to other terms. For the particular case in which only two load sources are considered, Eq. 22 becomes

$$
T\left\{\left[v_{1} \sum_{i_{1}}^{k_{1}} P_{i_{1}, 0}+v_{2} \sum_{i_{2}}^{k_{2}} P_{0, i_{2}}\right]+v_{1+2} \sum_{i_{1}}^{k_{1}} \sum_{i_{2}}^{k_{2}} P_{i_{1}, i_{2}}\right\}
$$

The crossing rates $v_{i+j}, v_{i+j+k}, \ldots, v_{1+2+\ldots+N}$ may be established with the aid of the technique developed by Winterstein.

\section{ACKNOWLEDGEMENT}

This work was supported by Brookhaven National Laboratory under Grant No. BNL-517722-S. 


\section{REFERENCES}

1. Shinozuka, M., "Notes on the Combinations of Random Loads (I)." July 1980, Columbia University Technical Report submitted to the Brookhaven National Laboratory under Grant No. BNL-517722-S. 
NRC FORM 335

$(7.77)$

U.S. NUCLEAR REGULATORY COMMISSION

BIBLIOGRAPHIC DATA SHEET

4. TITLE AND SUBTITLE (Add Volume No, if appropriate)

Probability Based Load Criteria for the Design of Nuclear

Structures: A Critical Review of the State-of-the-Art

7. AUTHOR(S)

M. Shinozuka, B.R. Ellingwood, P.C. Wang, C. Meyer, et al.

9. PERFORMING ORGANIZATION NAME AND MAILING ADDRESS (Include Zip Code)

Brookhaven National Laboratory

Upton, NY 11973

1. REPORT NUMBER (Assigned by DDC)

NUREG/CR-1979

BNL-NUREG- 51356

2. (Leave blank)

3. RECIPIENT'S ACCESSION NO

5. DATE REPORT COMPLETED \begin{tabular}{l|l}
\hline MONTH & YEAR
\end{tabular} February 198] DATE REPORT ISSUEO \begin{tabular}{l|l|}
\hline MONTH & YEAR \\
\hline APYII
\end{tabular} April 1981

6. (Leave blank)

8. (Leave blank)

12. SPONSORING ORGANIZATION NAME AND MAILING ADDRESS (include Zip Code)

U.S. Nuclear Regulatory Commission

Division of Reactor Safety Research

Washington, DC 20555

10. PROJECT/TASK/WORK UNIT NO.

11. CONTRACT NO.

NRC FIN NO. A3226

13. TYPE OF REPORT

Forma 1, Technical

June 1980 - December 1980

15. SUPPLEMENTARY NOTES

14. (Leave olank)

16. ABSTRACT (200 words or less)

Structures for nuclear power plant facilities must be designed to withstand safely and effectively all kinds of loads and load combinations that may be expected to occur during their lifetime. The traditional methods of structural design attempt to account for the inevitable variability in the loads, material strengths, in-service environments, and fabrication process, etc., through the use of safety factor, allowable stresses or load and resistance factors. This resport presents:

(a) An in-depth review of the state-of-the-art pertaining to probability-based analysis and design of civil engineering structures,

(b) A basis for extending existing probability-based methods to seismic Category I structures, and

(c) The availability of the pertinent data required to perform probabilistic analysis for seismic Category I structures.

\begin{tabular}{|l|l|}
\hline $\begin{array}{l}\text { 19. SE CURITY CLASS (This report) } \\
\text { UnClaSSified }\end{array}$ & 21. NO. OF PAGES \\
\hline 20. SECURITY CLASS (This page) & $\begin{array}{c}\text { 22. PRICE } \\
\text { S }\end{array}$ \\
\hline
\end{tabular}

